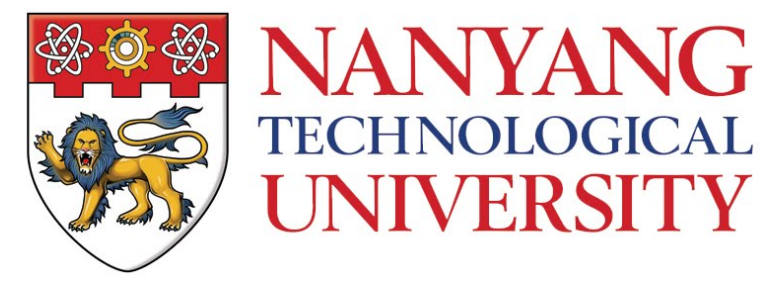

\title{
MODELLING SLUM DYNAMICS USING AN INTEGRATED AGENT BASED SIMULATION FRAMEWORK
}

\section{DEBRAJ ROY}

School of Computer Science and Engineering

A thesis submitted to the Nanyang Technological University in partial fulfilment of the requirement for the degree of Doctor of Philosophy 



\section{Acknowledgements}

I would like to express my sincere gratitude to my supervisor, Asst Prof. Bo An for his incredible understanding, help and support in a very difficult situation. I would also like to thank my co-supervisor and mentor, Dr. Michael Lees for giving me the opportunity and the inspiration to pursue my dreams. He has always been a reliable and uncompromising critic who has been there to help, advise and discuss about everything ranging from my research to teaching to technology and life in general. The weekly team meetings over coffee and, more recently, over hangouts, and his emphasis on encouraging everyone in his team to discuss their projects with each other not only helped me develop a lot of my ideas but made $\mathrm{PhD}$ life a lot more interesting when things got boring; and bearable when the going got tough. I am grateful to Dr. Karin Pfeffer for her guidance and contributions in my research and helping me collect data from India.

Prof. Peter Sloot has my sincerest gratitude for taking time out of his busy schedule to give me valuable feedback, advise and support for my research. I would also like to thank the members of my Thesis Advisory Committee - Prof. Cheong Siew Ann and Prof. Cai Wentong for their support and valuable feedbacks during the course of my doctorate studies.

I would also like to express my sincere gratitude to the School of Computer Science and Engineering, The Computational Intelligence Lab and the Parallel and Distributed Computing Center for providing me with the opportunity, the funds and, above all, the excellent facilities to pursue my doctorate studies. A special thanks is also due to Irene (Ng-Goh Siew Lai) and Kesavan Asaithambi for their excellent support with the resources at the laboratory.

Finally, I would like to thank my family for their unflinching support and belief in me; and my wife Ria Ghosh for listening to my constant complaints and rants, and always being there to cheer me up. 


\section{Contents}

List of Figures $\quad$ viii

List of Tables $\quad$ xii

1 Introduction 1

1.1 Research Salience . . . . . . . . . . . . . . . . . 2

1.2 Research Framework . . . . . . . . . . . . . . . . . 3

1.3 Research Goals and Key Contributions . . . . . . . . . . . . . . . 5

1.4 Dissertation Structure . . . . . . . . . . . . . . . . 6

1.5 Scope and Limitations . . . . . . . . . . . . . . . . . . . . 8

2 Slums Definitions and Characteristics $\quad 9$

2.1 Definition of Slums . . . . . . . . . . . . . . . . . . . 9

2.1.1 Definition by The Cities Alliance . . . . . . . . . . . . 10

2.1.2 Registrar General and Census Commissioner of India . . . . . . . . . . 10

2.1.3 Definition by The Karnataka Slum Areas Act . . . . . . . . . . . . 11

2.1 .4 Definition by UN Habitat . . . . . . . . . . . . . . . . . 11

2.1.5 Impact of Multiple Definitions of Slum . . . . . . . . . . . . . . . . . 12

2.2 Characterization of Slums . . . . . . . . . . . . . . . . . 13

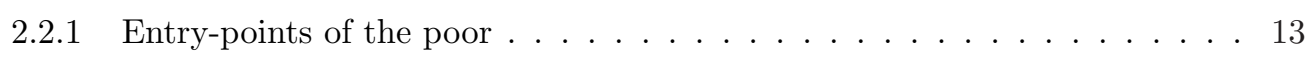

2.2 .2 Safety concerns of Women . . . . . . . . . . . . . . 14

2.2.3 Health and Child Mortality . . . . . . . . . . . . . . . . 14

2.2 .4 Lack of Education . . . . . . . . . . . . . . . . . . . . 14

2.2.5 Exclusion from formal financial system . . . . . . . . . . . 15

2.2.6 Political and Social Exclusion . . . . . . . . . . . . . . . . 15

2.3 Identifying Growth factors of Slums . . . . . . . . . . . . . . . . 15

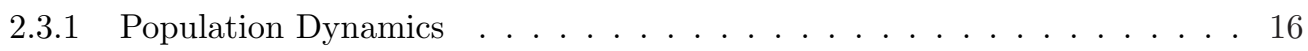


2.3.2 Economic Growth . . . . . . . . . . . . . . . . . 16

2.3.3 Housing Market Dynamics . . . . . . . . . . . . . . . . . . . . . . 17

2.3.4 Informal Economy . . . . . . . . . . . . . . . . . . . . . . 17

2.3.5 Local Topography . . . . . . . . . . . . . . . . . . . . . . 18

$2.3 .6 \quad$ Street Pattern . . . . . . . . . . . . . . . . . . . . . 18

2.3.7 The politics of Slums . . . . . . . . . . . . . . . . . . 19

2.4 Discussion . . . . . . . . . . . . . . . . . . . . . . . . . . . 19

3 Review of slum models $\quad 21$

3.1 Developing a framework for evaluating slum models . . . . . . . . . . . . . 22

3.1 .1 Spatial Resolution . . . . . . . . . . . . . . . . . . . . 23

3.1 .2 Temporal Resolution . . . . . . . . . . . . . . . . . . . . . 23

3.1.3 Level of Abstraction . . . . . . . . . . . . . . . . . . . . . . . . . 24

3.1.4 Growth Phenomenon . . . . . . . . . . . . . . . . . . . . . . . 24

3.1 .5 Sensitivity Analysis . . . . . . . . . . . . . . . . . . 24

3.1.6 Validation and Calibration . . . . . . . . . . . . . . 24

3.2 Taxonomy of Slum Models . . . . . . . . . . . . . . . . . . . . . . 25

3.3 Description of existing Slum Models _ . . . . . . . . . . . . . . . . . . . . 26

3.3.1 Informal Settlement Growth Model (ISGM) . . . . . . . . . . . . 26

3.3 .2 The Peripherization Model . . . . . . . . . . . . . . . . . . . . . . . . 29

3.3.3 Informal Settlement Growth Pattern Model . . . . . . . . . . . . . . . . . 31

3.3.4 Manzese Settlement Growth Model . . . . . . . . . . . . . . . . . . . . . . 33

3.3.5 Economic Disparity Model . . . . . . . . . . . . . . . . . . . 34

3.3 .6 Slumulation . . . . . . . . . . . . . . . . . . . . . . . . . 37

3.4 Discussion . . . . . . . . . . . . . . . . . . . . . . 39

4 Slums in Bangalore $\quad 41$

4.1 Local Governance and Distribution of Slums in Bangalore . . . . . . . . . . . 42

4.1 .1 Local governance structure of Bangalore . . . . . . . . . . . . . . . . 42

4.1.2 Spatial Distribution of Slums in Bangalore . . . . . . . . . . . . . . . 42

4.1 .3 Spatial clustering of Slums in Bangalore . . . . . . . . . . . . . . 45

4.1.4 Status of low-cost housing in Bangalore . . . . . . . . . . . . . . . . 46

4.2 Status of Slums in Bangalore using the Survey Data . . . . . . . . . . . . . . 46

4.2.1 Demographic Profile of Households . . . . . . . . . . . . . . . . . . 50 


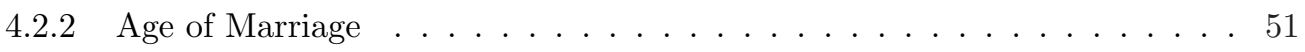

4.2 .3 Literacy and Occupation . . . . . . . . . . . . . . . 52

4.2 .4 Income Distribution . . . . . . . . . . . . . . . . . . 54

4.2.5 Expenditure distribution . . . . . . . . . . . . . . . . 57

4.2.6 Status of Welfare Benefits . . . . . . . . . . . . . . 58

4.2.7 Housing Status and Security of Tenure . . . . . . . . . . . . . 59

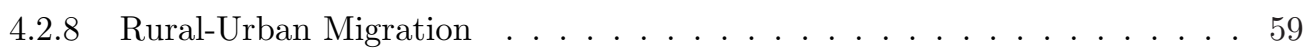

4.2 .9 Physical Infrastructure in Slums . . . . . . . . . . . . . . . 59

4.2 .10 Choice of Slums . . . . . . . . . . . . . . . . . 60

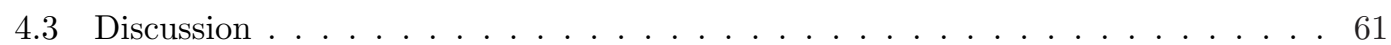

5 Multivariate Statistical Modelling of Segregation in the Slums of Bangalore. 63

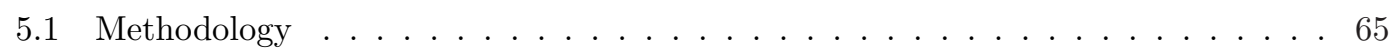

5.1.1 Theoretical Framework . . . . . . . . . . . . . . 65

5.1 .2 Quantitative Methods ................. 66

5.1 .2 .1 Index of Dissimilarity . . . . . . . . . . . 66

5.1.2.2 Correspondence Analysis . . . . . . . . . . . . . 67

5.1.2.3 Stepwise Regression . . . . . . . . . . . . . . 68

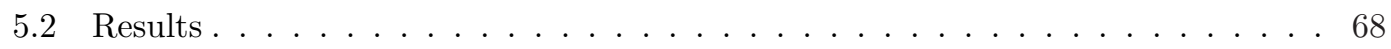

5.2 .1 Degree of Segregation . . . . . . . . . . . . . . 68

5.2 .2 Spatial Segregation based on Religion . . . . . . . . . . . . 69

5.2 .3 Income Inequality and Poverty based on Religion . . . . . . . . . . . . 70

5.2.4 Opportunity Bias based on Religion . . . . . . . . . . . . 74

5.2.5 Satisfaction Based on Religion . . . . . . . . . . . . . 77

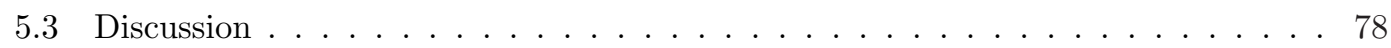

6 Population Dynamics Model $\quad 81$

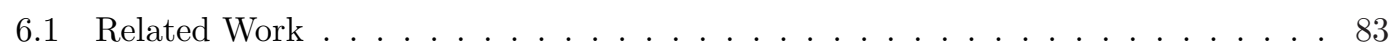

6.2 The Model . . . . . . . . . . . . . . . . . . . . 85

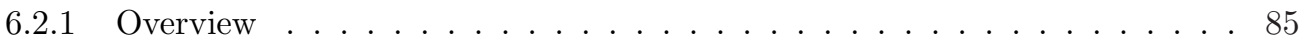

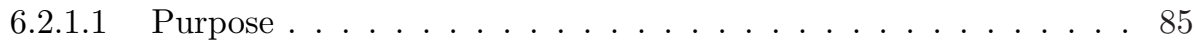

6.2.1.2 State Variables and Scales . . . . . . . . . . . . . 86

6.2.1.3 Process Overview and Scheduling . . . . . . . . . . 86

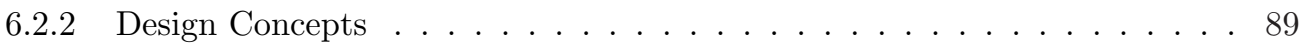


$6.2 .2 .1 \quad$ Emergence . . . . . . . . . . . . . . . . . . 89

6.2.2.2 Adaptation . . . . . . . . . . . . . . . . . . . 90

$6.2 .2 .3 \quad$ Fitness . . . . . . . . . . . . . . . . . 90

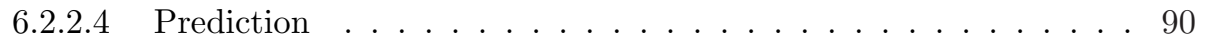

6.2 .2 .5 Interaction $\ldots \ldots \ldots \ldots \ldots \ldots \ldots \ldots \ldots \ldots \ldots \ldots$

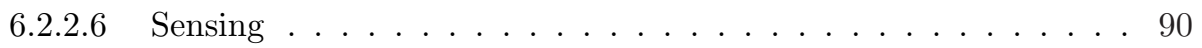

6.2.2.7 Stochasticity . . . . . . . . . . . . . . . . . 91

6.2 .2 .8 Collectives . . . . . . . . . . . . . . . . . . . . 91

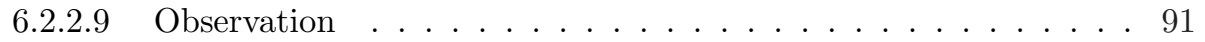

6.2 .3 Details . . . . . . . . . . . . . . . . . . . . . . . . 92

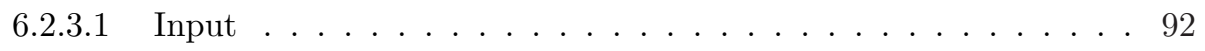

6.2.3.2 Initialization . . . . . . . . . . . . . . . . 93

6.2.3.3 Calibration of Dynaslum . . . . . . . . . . . . . . 93

6.3 Validation and Sensitivity Analysis . . . . . . . . . . . . . . . . . . . . 94

6.3 .1 Validation of Model Initialisation . . . . . . . . . . . . . . . 95

6.3.2 Validation of Model Steps . . . . . . . . . . . . . . . . . . 96

6.3 .3 Sensitivity Analysis . . . . . . . . . . . . . . . . . . . . 96

6.3.3.1 Sobol' Indices . . . . . . . . . . . . . . . . . . . 97

6.3.3.2 One Factor At a Time $($ OFAT) $\ldots \ldots \ldots$. . . . . . . 98

6.3.3.3 Sensitivity of Time Step on Model Output . . . . . . . . . . 98

6.4 Results . . . . . . . . . . . . . . . . . . . . . . . . . . . . . . . . . 99

6.4.1 Difference in HLC between a slum and non-slum household . . . . . . . . 100

6.4 .2 Impact of Home-Leaving on HLC . . . . . . . . . . . . . . . . . . . . . 102

6.4.3 Impact of Couple Formation on HLC . . . . . . . . . . . . . . . . . . 104

6.5 Discussion . . . . . . . . . . . . . . . . . . . . . . . 105

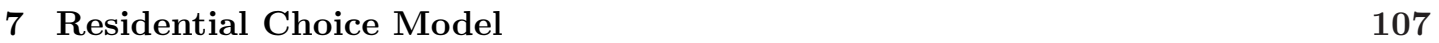

7.1 Related Work . . . . . . . . . . . . . . . . . . . . . . . . . . . . 109

7.1.1 The Choice and Bid-Auction Approach . . . . . . . . . . . . . . 109

7.1.1.1 Estimating Bid Function . . . . . . . . . . . . . . 111

7.1.2 Microsimulation models . . . . . . . . . . . . . . . . . . . . . 113

7.2 The Model . . . . . . . . . . . . . . . . . . . . . . . . . . . . . . . 114

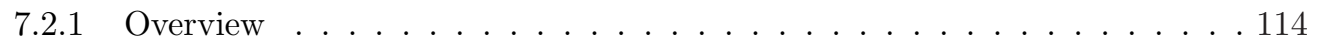


7.2 .1 .1 Purpose . . . . . . . . . . . . . . . . . 114

7.2.1.2 Entities, state variables, and scales . . . . . . . . . . . . . 114

7.2.1.3 Process overview and scheduling . . . . . . . . . . . . 117

7.2 .2 Design Concepts . . . . . . . . . . . . . . . . . . . . . . . . 117

7.2.2.1 Theoretical Background . . . . . . . . . . . . . . . . 117

7.2.2.2 Concepts And Theories . . . . . . . . . . . . . . . . 118

7.2 .2 .3 Individual Decision-Making . . . . . . . . . . . . . . 120

7.2 .2 .4 Individual Sensing . . . . . . . . . . . . . . . . . . 122

7.2 .2 .5 Interaction . . . . . . . . . . . . . . . . . 123

7.2.2.6 Individual Prediction . . . . . . . . . . . . . . . . . 125

7.2 .2 .7 Collectives . . . . . . . . . . . . . . . . . 126

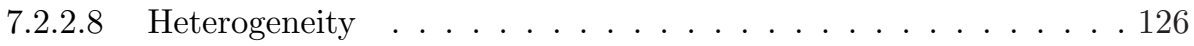

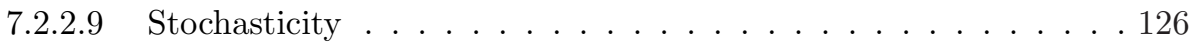

$7.2 .3 \quad$ Details . . . . . . . . . . . . . . . . . . . . . . . . . 126

7.2.3.1 Agent Population . . . . . . . . . . . . . . . . . 127

7.2.3.2 Estimating Agents' satisfaction levels . . . . . . . . . . . . . . 127

7.3 Validation and Sensitivity Analysis . . . . . . . . . . . . . . . . . . 129

7.3.1 Constant Population and Constant Labour Market . . . . . . . . . . . . . 130

7.3.2 Increasing Population and Constant Labour Market . . . . . . . . . . . 132

7.3.3 Increasing Population and Dynamic Labour Market . . . . . . . . . . . 133

7.3 .4 Sensitivity analysis . . . . . . . . . . . . . . . . . 135

7.4 Results . . . . . . . . . . . . . . . . . . . . . . . . 137

7.4.1 The Effect of Social Satisfaction on Residential Choice . . . . . . . . . . . 138

7.4.2 The Effect of Jobs on Residential Choice . . . . . . . . . . . . . . . . . 139

7.5 Discussion . . . . . . . . . . . . . . . . . . . . . . . . . . . . . 141

7.5.1 Limitations of the Model . . . . . . . . . . . . . . . . . . . . . . 142

8 Conclusion and Future Work $\quad 143$

8.1 Summary of Findings . . . . . . . . . . . . . . . . . . . 145

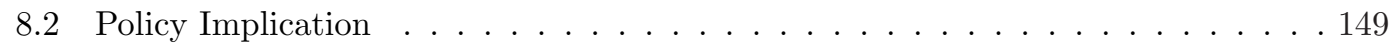

8.3 Future Extension . . . . . . . . . . . . . . . . . . . . . . . . . 150

$\begin{array}{ll}\text { A Classifications in the Survey Data } & 153\end{array}$ 
Bibliography

165 


\section{List of Figures}

3.1 Simulation Results (Year 1976, 1981, 1986, 1991, 1996 and 2001 shown in legends) from ISGM Model. Source: Sietchiping 2004. . . . . . . . . . . . . . . 28

3.2 Simulation Results from Peripherization Model. Source: Barros 2012. . . . . . . 30

3.3 Simulation Results from ISGPM Model. Source: Vincent 2009. . . . . . . . . . . 33

3.4 Results from 1987-1993, empirical data on left, simulated data on right. Source: Augustine-Beckers et al 2011. . . . . . . . . . . . . . . . . 35

3.5 Simulation Results from Economic Disparity Model. Source: Felsen, M. and Wilensky, U. 2007. . . . . . . . . . . . . . . . 36

3.6 Slumulation Output. Source: Patel 2012. . . . . . . . . . . . . . . 38

4.1 Governance structure in the city of Bangalore. . . . . . . . . . . . . . . . 43

4.2 Slum Distribution across Bangalore in 2005 (Source: Karnataka Slum Development Board). . . . . . . . . . . . . . . . . . . . . 44

4.3 Spatial Normal Distribution of Slums across Bangalore in 2005 (Source: Karnataka Slum Development Board). . . . . . . . . . . . . . . . . . 44

4.4 Spatial Clustering of Slums in Bangalore in the year 2005. . . . . . . . . . . . . 47

4.5 Hot Spots of Slums in Bangalore in the year 2005. . . . . . . . . . . . . . . . . . 48

4.6 Age Distribution across the 37 slums (Refer table A.2 for list of slums) in Bangalore. 50

4.7 Household Size Distribution across the 37 slums (Refer table A.2 for list of slums)

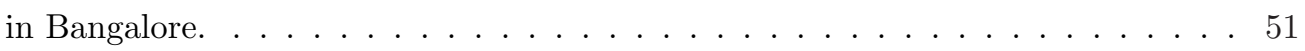

4.8 Age Distribution of Marriage for men in slums of Bangalore. . . . . . . . . . . . 52

4.9 Age Distribution of Marriage for women in slums of Bangalore. . . . . . . . . . 53

4.10 Education Levels across age groups in the 37 slums of Bangalore. . . . . . . . . . 54

4.11 Occupation Categories across age groups in the 37 slums of Bangalore. . . . . . . 55

4.12 Income Distribution of Slum Dwellers in the 37 slums of Bangalore. . . . . . . . 55 
4.13 Income based on religion (1 - Hinduism, 2- Muslim, 3- Christian) and Occupation(1 - Professional White Collar, 2- Professional Blue Collar, 3- Small Enterprises, 4-Self Employed - Sales, 5 - Self Employed - Repair, 6 - Home Based, 7 - Domestic Services, 8 - Small Industry, 9 - Security, 10- Transport Services, 11 - Construction, 12 - manual labour, 13 - Others $) \ldots \ldots 56$

4.14 Expenditure distribution of slum households on various categories. . . . . . . . 57

4.15 Asset ownership of slum households. . . . . . . . . . . . . . . . . . . 57

5.1 Religion Distribution across the 37 slums in Bangalore. . . . . . . . . . . . . . 69

5.2 CA of religion, Number of rooms and location of slums. . . . . . . . . . . 71

5.3 CA of language and list of slums in Bangalore. Language is categorised as (1 Kannada, 2-Tamil, 3 - Malayalam, 4-Telugu, 5 - Hindi, 6- English, 7 - Urdu, 8 - Bengali, 9 - Others $) \ldots \ldots \ldots \ldots 72$

5.4 CA of religion, income and family size. Income is categorised as follows (1 - 200 to $1300,2-1300$ to $2400,3-2400$ to $3500,4-3500$ to $4600,5-4600$ to 5700 , $6-5700$ to $8000,7-8000$ to $12000,8-12000$ to 18000,9 - Greater than 18000 INR). Religion is indicated as follows (1 - Hinduism, 2- Muslim, 3-Christian, 4 - Others). . . . . . . . . . . . . . . . . . . . . 73

5.5 CA scatter plot of religion and occupation category (1 - Professional White Collar, 2- Professional Blue Collar, 3- Small Enterprises, 4-Self Employed Sales, 5 - Self Employed - Repair, 6 - Home Based, 7 - Domestic Services, 8 - Small Industry, 9 - Security, 10- Transport Services, 11 - Construction, 12 -

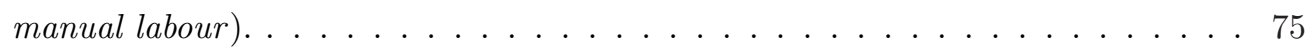

5.6 Spatial Distribution of Occupation Categories (1 - Professional White Collar, 2Professional Blue Collar, 3-Small Enterprises, 4-Self Employed - Sales, 5 - Self Employed - Repair, 6 - Home Based, 7 - Domestic Services, 8 - Small Industry, 9 - Security, 10- Transport Services, 11 - Construction, 12 - manual labour). . . 77

5.7 CA scatter plot of religion and satisfaction levels $\ldots \ldots \ldots \ldots$

6.1 Relative probability of birth at a given age $[1] \ldots \ldots \ldots$. . . . . . . . 91

6.2 Annual risk of death by age and $\operatorname{sex}[1] \ldots \ldots \ldots \ldots$

6.3 Household Age. . . . . . . . . . . . . . . . . . . . . . . . 95

6.4 Household Size. . . . . . . . . . . . . . . . . . . . . . . . . 95

6.5 Simulated HLC of slums in Dynaslum. . . . . . . . . . . . . . . . 96 
6.6 Actual HLC from real data of slums in Karnataka, year 2005 [2] . . . . . . . . 96

6.7 Sensitivity of Immigration Rate w.r.t. HLC. . . . . . . . . . . . . . . . . 99

6.8 Sensitivity of Growth Rate w.r.t. HLC. . . . . . . . . . . . . . . . . 99

6.9 Sensitivity of Home Leaving w.r.t. HLC. . . . . . . . . . . . . . . . . . 99

6.10 Sensitivity of Marriage Rate w.r.t. HLC . . . . . . . . . . . . . . . 99

6.11 Sensitivity Analysis of Dynaslum parameters with respect to HLC. . . . . . . . . 99

6.12 Total population over 30 runs. . . . . . . . . . . . . . . . . 100

6.13 Gender ratio over 30 runs. . . . . . . . . . . . . . . . . . . . . . 100

6.14 Peak mean Household size over 30 runs. . . . . . . . . . . . . . . . . . 100

6.15 Mean and Standard deviation of Total Population and Gender ratio with different time steps. . . . . . . . . . . . . . . . . . . . 100

6.16 HLC in non-slum households of Karnataka in the year 1998 [3] . . . . . . . . . 101

6.17 HLC in non-slum households of Karnataka in the year 2005 [2] . . . . . . . . 101

6.18 Simulated Household Life Cycle: $\delta_{g}=0.05, P_{h}=0.05 \ldots \ldots \ldots 2$

6.19 Simulated Household Life Cycle: $\delta_{g}=0.05, P_{h}=0.025 \ldots \ldots$. . . . . . 102

6.20 Resource consumption in slums of Bangalore from survey data. . . . . . . . . . 103

6.21 Living space per capita in slums of Bangalore from survey data. . . . . . . . . . 103

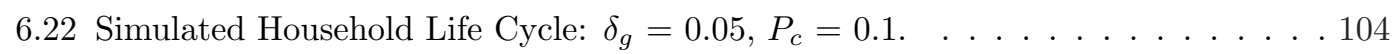

6.23 Simulated Household Life Cycle: $\delta_{g}=0.03, P_{c}=0.6 \ldots \ldots$. . . . . . 104

7.1 Decision making process of an individual agent (see section 7.2.2.3) Each block contains the section where the process is explained in details. . . . . . . . 116

7.2 Vector model representation. . . . . . . . . . . . . . . . . . . . . . 121

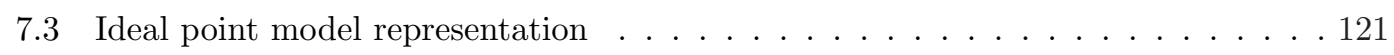

7.4 Actual Degree Distribution based on 1107 households from 37 slums in Bangalore. 124

7.5 Actual Average Connection degree based on 1107 households from 37 slums in Bangalore. . . . . . . . . . . . . . . . . . . . . . . . . . . . . . . . . . . 124

7.6 A 2- Simplex showing the possible values of $\omega \ldots \ldots$. . . . . . . . 128

7.7 A ternary diagram showing the possible distribution of $\omega$ using Dirichlet distributions. . . . . . . . . . . . . . . . . . . . . . . 130

7.8 Social Mobility of the Residential Choice Model under Constant population and Constant Labour Market. . . . . . . . . . . . . . . . . . . . . . . . . 131 
7.9 Social Mobility of the Residential Choice Model under Dynamic population and Constant Labour Market. . . . . . . . . . . . . . . . . . . . . . . 133

7.10 Social Mobility of the Residential Choice Model under Dynamic population and Dynamic Labour Market. . . . . . . . . . . . . . . . . . . . . . . . 134

7.11 Simulated Distribution of Commuting Distance. . . . . . . . . . . . 135

7.12 Observed Distribution of Commuting Distance (1107 slum households). . . . . . 135

7.13 Simulated Degree Distribution. . . . . . . . . . . . . . . . 135

7.14 Average Connection degree. . . . . . . . . . . . . . . . . . . 135

7.15 SA showing the impact of the parameter $\omega$ on Gender segregation. . . . . . . 136

7.16 SA showing the impact of the parameter $\omega$ on Religion segregation. . . . . . . 136

7.17 SA showing the impact of the parameter $\omega$ on Language segregation. . . . . . . . 136

7.18 SA showing the impact of the parameter $\omega$ on Occupation Bias. . . . . . . . . 136

7.19 Impact of Social Satisfaction using Residential Mobility on Muslims. . . . . . . . 137

7.20 Impact of Social Satisfaction using Residential Mobility on Hindus. . . . . . . . . 137

7.21 Distribution of Homophily Degree among Muslims. . . . . . . . . . . . . . . . 137

7.22 Distribution of Homophily Degree among Hindus. . . . . . . . . . . . . . . . . 137

7.23 Distribution of Occupation Categories. . . . . . . . . . . . . . . . . . 139

7.24 Distribution of Languages. . . . . . . . . . . . . . . . . . . . . . . . . . 139

7.25 Simulation Slum Migration: State of a slum at $t=0$. The size of the circle represents the number of slum dwellers in the slum. . . . . . . . . . . . . . 140

7.26 Simulation Slum Migration: State of slum at $t=8$. Slum dwellers migrate (due to job loss) and form a new slum nearby for new opportunities . . . . . . . . 140

7.27 Simulation Slum Migration: State of slum at $t=12$. Slum dwellers migrate (due to job loss) and form a new slum nearby for new oppurtunities. . . . . . . . . . 140

7.28 Simulation Slum Migration: State of slum at $t=15$. New slum dwellers migrate to the previous slums for new opportunities . . . . . . . . . . . . . . 140

7.29 Emergence and Growth of a slum in Hoodi, Bangalore (marked with a red polygon). a) Slums emerge near a construction Site in 2008. b) Slum grows near the same site. c) Slum disappear when construction is complete in 2013. d) A slum re-emerge at the same site in 2014 (IMAGE SOURCE: Google Earth). . . . . . . 141

8.1 Schematic Diagram Dynaslum . . . . . . . . . . . . . . . . . . . . . 150 


\section{List of Tables}

3.1 Comparison and synopsis of dynamic models on informal settlements . . . . . . . 27

4.1 Comparison of expected and observed number of slums to prove SND slums in Bangalore. . . . . . . . . . . . . . . . . . . 45

4.2 Gender and Age Distribution across the 37 slums (Refer table A.2 for list of slums) in Bangalore. . . . . . . . . . . . . . . . . . . . 50

4.3 Comparison of CPI between slums and non-slum households. Source:Central Statistics Office, February 11, $2014 \ldots \ldots$. . . . . . . . . . 58

4.4 Housing types based on construction material. . . . . . . . . . . . . . 58

4.5 Reason for wanting to change current slum settlement. . . . . . . . . . . . 60

4.6 Reason for moving into current slum. . . . . . . . . . . . . . . . 60

4.7 Reason for migrating into slums of Bangalore. . . . . . . . . . . . . . . . . 60

5.1 Formation of dichotomous groups. . . . . . . . . . . . . . 67

5.2 Index of Dissimilarity f . . . . . . . . . . . . . . . . . 68

5.3 Correlation Matrix of Dissimilarity Index Based on 5 parameters. . . . . . . . . . 69

5.4 Average Slum Age based on their Location . . . . . . . . . . . . . . 70

5.5 Stepwise regression analysis of factors influencing fertility among Muslims in Slums of Bangalore. . . . . . . . . . . . . . . . . . 72

5.6 Preference of male child and its association with fertility among Muslims in Slums of Bangalore. $N_{M}$ and $N_{F}$ are Number of male and female child in first two live births. $\mu_{B}$ is the Mean live birth. Pearson coefficient shows a negative correlation with the number of sons in first two live births with fertility, at the same time showing a positive correlation with number of female child in the first two live births . . . . . . . . . . . . . . . . . . . 74

5.7 Sources of Finance for Self-Employment . . . . . . . . . . . . . . . 75 
6.1 Dynaslum Model Parameters . . . . . . . . . . . . . . . . . . . 87

6.2 Age Distribution. . . . . . . . . . . . . . . . . . . . . . . . . 92

6.3 Religion Distribution. . . . . . . . . . . . . . . . . . . . 92

6.4 Language Distribution. . . . . . . . . . . . . . . . . . . . . . . . . . . 92

6.5 Household Size Distribution. . . . . . . . . . . . . . . . . . . . . 92

6.6 Simulated slum population (averaged over 100 runs) vs Actual slum population in Bangalore. $N_{A c t}$ and $N_{\text {Sim }}$ represents the total actual and simulated slum population in Bangalore. $\varphi_{\text {Act }}$ and $\varphi_{\text {Sim }}$ represents the actual and simulated sex ratio (male to female ratio) in slums of Bangalore. The standard deviation (SD) is also shown in parenthesis . . . . . . . . . . . . . . . . . . . 95

6.7 Model parameters and range for sensitivity analysis . . . . . . . . . . . . . 97

6.8 First order and total-order Sobol' sensitivity indices . . . . . . . . . . . . . 97

7.1 Interaction of Spatial Attribute and Household Attribute for Latent Auction Model.112

7.2 Estimation of DCM coefficients for slums in Bangalore. . . . . . . . . . . . 113

7.3 Comparison of Microsimulation Model Characteristics. . . . . . . . . . . . . . 115

7.4 Comparison of Index of Dissimilarity calculated based on the simulation output under 4 different condition: $\omega_{s o c}=1, \omega_{e n v}=1, \omega_{e c o}=1, \omega_{s o c}+\omega_{e c o}+\omega_{e n v}=$

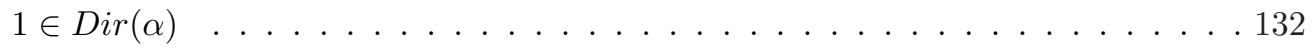

8.1 Growth factors included in Dynaslum. $\rho_{P}$ - Population Dynamics, $\rho_{E}$ - Economic Growth, $\rho_{I}$ - Informal Economy, $\rho_{G}$ - Politics Of Slum, $\rho_{H}$ - Housing Dynamics, $\rho_{T}$ - Local Topography, $\rho_{S}$ - Street Pattern . . . . . . . . . . . . . 144

A.1 Classification of jobs into Occupation Category . . . . . . . . . . . . . 153

A.2 Classification of Income into Income Category . . . . . . . . . . . . . . . . . . 154

A.3 List of slums surveyed . . . . . . . . . . . . . . . . . . . . . . 155

B.1 Sources of water supply in the Slums of Bangalore . . . . . . . . . . . . 157

B.2 Sources of electricity in the Slums of Bangalore . . . . . . . . . . . . . . . . 158

B.3 Toilet facilities in the Slums of Bangalore . . . . . . . . . . . . . . . 158

B.4 Kitchen types in the Slums of Bangalore . . . . . . . . . . . . . . . 158

B.5 Bathroom types in the Slums of Bangalore . . . . . . . . . . . . . . . 159

B.6 Roof and Wall materials used in the Slums of Bangalore . . . . . . . . . . . . . . 159

B.7 Floor materials used in the Slums of Bangalore . . . . . . . . . . . . . . . 159 
C.1 State Variable for a Individual . . . . . . . . . . . . . . . . . . . . . . 161

C.2 Explanation of State Variable for a Slum Household . . . . . . . . . . . . . . 162

C.3 State Variable of Jobs ． . . . . . . . . . . . . . . . . . . . . . . . . . . . 162

C.4 State variable for a Slum . . . . . . . . . . . . . . . . . . . 163 


\section{List of Equations}

5.1 Index of Dissimilarity. . . . . . . . . . . . . . . . . . 66

7.1 Random Utility Model. . . . . . . . . . . . . . . . . . . . . . . . . 109

$7.2 \quad$ Utility constraints. . . . . . . . . . . . . . . . . . . . . . . 109

7.3 Probability of an household $h$ relocation from location $j$ to $i$. . . . . . . . . . . . 109

7.4 Probability of an household $h$, choosing a location $i$ from a set $s$. . . . . . . . 110

7.5 Probability of a household $h$ choosing a location $i$. . . . . . . . . . . . . . . 110

7.6 The highest bid or rent $\left(r_{i}\right) \ldots \ldots \ldots \ldots \ldots \ldots$

7.7 Overall satisfaction of agent $\left.S_{\text {overall }}(\mathrm{a})\right) \ldots \ldots \ldots \ldots \ldots$

7.8 Social, economic and environmental satisfaction of agent $a$. . . . . . . . . . . . 120

7.9 Vector model formulation. . . . . . . . . . . . . . . . . . . . . . . . 121

7.10 Ideal Point model formulation. . . . . . . . . . . . . . . . . . . . . . . . . . 121

7.11 Social state of an individual agent $\left(P_{\text {soc }}(a)\right) \ldots \ldots \ldots \ldots \ldots$. . . . . . . . . . . . . . .

7.12 Economic state of an individual agent $\left(P_{\text {eco }}(a)\right) \ldots \ldots \ldots$. . . . . . . . . 122

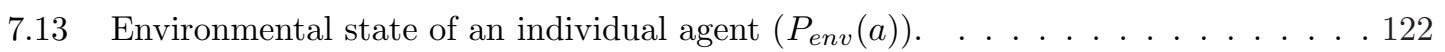

7.14 Degree of interactibility of two agents $(a$ and $b)$. . . . . . . . . . . . . 123

7.15 Neighbourhood of two agents $(a$ and $b)$. . . . . . . . . . . . . . 123

7.16 Sector of two agents $(a$ and $b) \ldots \ldots \ldots \ldots \ldots \ldots$

7.17 Degree of homophily between two agents $(a$ and $b) \ldots \ldots$. . . . . . . . . . . 124

7.18 Probability of an edge between two agents $(a$ and $b) \ldots \ldots$. . . . . . . . . 124

7.19 Probability of agent $a$ updates its information. . . . . . . . . . . . . 125

7.20 Probability of agent $a$ following agent $b \ldots \ldots \ldots \ldots$

7.21 Probability of agent $a$ adopting agent $b \ldots \ldots \ldots \ldots \ldots$

7.22 Probability of agent $a$ individual processing. . . . . . . . . . . . . . 126

7.23 Probability of agent changing to $A_{c t}$. . . . . . . . . . . . . . 126

7.24 Constraint of multinomial distribution. . . . . . . . . . . . . . . . . . . . 128 


\begin{abstract}
Today, over half of the world's population lives in urban areas and by the middle of this century 7 out of 10 people will live in a city. This increased urbanization has also lead to more and more people residing in informal settlements generally known as slums. In India, for instance, roughly 13.7 million households, or $17.4 \%$ of urban Indian households, are considered to reside in slums. Slums, are complex dynamic systems that have close symbiotic relationships with their encompassing cities. In order to identify the intricate consequences of particular policies, governments must consider and understand the interactions among multitude of factors. The relationship between a slum and its parent city can be commensalistic or parasitic. Specifically, in some cases, the economic and political power of slums is so significant that cities need to maintain them. The traditional view of slums, certainly from a policy and research perspective, is that these were controlled systems, maintained in an equilibrium state by negative feedbacks. The new, complexity view, considers slums as dynamic, non-equilibrium systems that are constantly changing and adapting. It is thus essential to examine the practical questions related to slum formation for informed policy-making: (i) what are the differences between slum and non-slum urban households? (ii) Where and when are they formed? and (iii) What are the key factors considered by slum households when choosing a slum?

This dissertation describes a novel slum growth model, namely Dynaslum that will contribute to strategic slum planning and management solutions. Dynaslum is designed to integrate Agent-Based Modelling (ABM), Discrete Choice Theory (DCT), and Geographic Information System (GIS) within a single framework. ABM coupled with DCT will provide a framework to study household dynamics and residential choices of slum inhabitants while GIS will provide the ability to create a multiscale spatial environment. Dynaslum will explore the interaction between the slum households and the encompassing spatial configuration of the city to predict the emergence of slum patterns.
\end{abstract}


Dynaslum is implemented based on a case study of Bangalore, an Indian city which has experienced high levels of urbanisation and rural-urban migration in the past decade. We have used a novel and unique dataset based on the field work from 37 slums in Bangalore combined with the NFHS data to calibrate Dynaslum and validate our findings. This dissertation presents the following key insights to address and understand the growth and emergence of slums. First, we investigate Tilly's theory on group segregation and show how segregation reproduces or reinforce inequality within the slums of Bangalore using statistical modelling (correspondence analysis and regression). Second, we find that high rate of home leaving among young adults is the key determinants for the large variation in the life cycle of slum households. We show that reducing home leaving among young adults will reduce the formation number of new slum households and contribute to a higher but stable household size. This will lead to efficiency and higher per capita resource consumption when building capacity for slum development (resettlement colonies) as policy makers would be able to plan for a stable household size. Finally, we demonstrate criticality in the process of inter-slum migration based on social and economic satisfaction. Further, we explore how creating jobs in different occupational categories across various social groups may impact the residential choices of slum dwellers.

Dynaslum serves as a decision support system for slums in general, that will help guide experts when evaluating or designing policies to improve conditions within slums. Such a model provides a virtual slum that decision-makers and researchers can use to explore how different policies would impact the growth, development or contraction of slums. 


\section{Chapter 1}

\section{Introduction}

Today, over half of the world's population lives in urban areas and by the middle of this century 7 out of 10 people will live in a city. This increased urbanization has also lead to more and more people residing in informal settlements generally known as slums. In India, for instance, roughly 13.7 million households, or $17.4 \%$ of urban Indian households, are considered to live in slums. In India alone this equates to over 200 million people. Over a third of India's slum population lives in 46 million-plus cities [1]. Likewise, many other countries around the world offer similar statistics and in fact the world's most notorious slums span multiple continents: Neza-Chalco-Itza (Mexico), Orangi Town (Pakistan), Dharavi (India), Khayelitsha (South Africa), and Kibera (Kenya). In spite of such a high incidence of slums in the less developed countries, research on the growth and emergence of slums is still in its infancy. Therefore, it is crucial to improve our understanding on the growth of slums in the context of less developed countries. Previous studies have employed various methodologies to understand the underlying dynamics of slum growth and emergence, but they have not produced adequate knowledge to design effective context specific slum policies [4]. Consequently, most slum policies have been implemented without proper planning tools and analysis.

This dissertation presents a novel computational simulation model named Dynaslum that could be applied to understand slum dynamics in the cities of less developed countries. Dynaslum is designed to explore the household dynamics and residential mobility of slum households in cities of the developing world with underlying complex non-linear socio-economic dynamics. The calibration and validation of Dynaslum is performed based on novel unique on-field survey and census datasets from the city of Bangalore in India. Dynaslum is developed to function as a decision support system for city development authorities and policy makers to assess 


\section{INTRODUCTION}

and evaluate policy interventions in a computer-generated environment. Furthermore, scientific researchers may also apply Dynaslum to study non-linear complex system properties (such as critical transitions, regime shifts, punctuated equilibria) and produce emerging insights for slum management policies in cities. Dynaslum further validates the significance of modelling household dynamics (household Life cycle) and individual behaviour (role of social capital in residential choice) explicitly when analysing slums as it may lead to new insights.

\section{$1.1 \quad$ Research Salience}

The emergence of slums, and growing number of people living in slums, is now a very significant and intricate global challenge for our society $[5,6]$. While there are many different definitions and classification criteria of what constitutes a slum [7], for residents the reality is often inadequate shelter, poor access to basic services such as water and sanitation, insufficient access to healthcare and in general a low quality of life. Slums are the by-product of social and economic impact due to rapid urbanization. Many nations including developed and developing countries together are formulating and organizing strategies to eradicate this problem. For example, in 2011 the United States introduced a bill to increase aid for "Shelter, Land and Urban Management (SLUM)" in developing countries, while the eight Millennium Development Goals (MDG) aimed to better the condition of slums by 2015 [8]. Despite the initiatives, the slum population continues to increase in the developing countries. The MDG 2015 report highlights the use of "data driven evidence-based decision-making" as the way forward for its post-2015 development agenda.

Policy intervention in slums is one of the key mechanisms adopted by various governments to enhance the quality of life of the urban poor. Slum policies have evolved and urban authorities have adopted different strategies, ranging from in-situ development in slums, relocation to the resettlement colonies, and to slum evictions. These policies were reviewed and found to be inefficient $[9,10]$. The reality, however, shows that the previous slum management policies were either experimental or incremental $[6,11]$. Incremental policy-making looks at existing programs or policies and uses the "Goodness of fit" criteria to implement change. They rely on past outcomes as a guide and are best suited to situations where there is high degree of continuity in the problem, which reduces the risks and costs of uncertainty. Experimental policy-making pertains to policies implemented without any substantial prior research. They are exploratory and are usually designed as part of government-sponsored pilot programs targeted at 
preselected groups. Both incremental and experimental policies have often been implemented without knowing their negative implications. There are numerous ex-post analyses of slum policies $[12,13$, e.g.] but very few attempts have been made to understand the impact of policies ex-ante. In India, an extensive low cost housing program for urban poor has been launched, known as Rajiv Awas Yojana or RAY [14]. RAY aims to provide basic amenities such as water system, waste disposal, internal and access roads, street lamps and various social infrastructure facilities in slums adopting a 'whole city' approach. Under the mandate of the National Resources Centre in New Delhi, surveys are currently being carried out to understand the differences in slum typologies and how slums can be classified based on their deficiencies and access to resources. A key challenge for policy makers in implementing RAY is to understand the slum typology and underlying driving forces as there is unlikely a 'one-size fits all' solution to the problem. Therefore, this dissertation describes a "data-driven evidence based" slum growth model, namely Dynaslum which will act as a decision support system for urban planners where they can conduct various policy experiments to understand the impacts ex-ante.

\subsection{Research Framework}

Slums, are complex dynamic systems that have close symbiotic relationships with their encompassing cities. In order to identify the intricate consequences of particular policies, governments must consider a multitude of factors, and most importantly understand how these factors interact. The relationship between a slum and its parent city can be commensalistic or parasitic. Specifically, in some cases, the economic and political power of slums is so significant that cities need to maintain them. Slums can be compared to a parasitic organism, thriving at the expense of the city and draining key urban resources. It grows and subdivides within itself much like an organism would when provided with restrictions. An alternate view is one where a slum could be commensalistic in nature as each slum in a city serves a specific function and provides cheap labour for the development of urban infrastructure. The traditional view of slums, certainly from a policy and research perspective, "is that these were controlled systems, maintained in an equilibrium state by negative feedbacks" [15]. The new, complexity view, considers slums as

"dynamic, non-equilibrium systems that are constantly changing and adapting" [15]. It is clear that slums have emerged "spontaneously from the interactions of the component parts" [15] (spatial components of shelter deprivation and the dynamics of slum development), not dictated 


\section{INTRODUCTION}

in a top-down manner. Viewing and analysing slums as complex systems should therefore lead to new perspectives on what is an increasingly challenging problem.

Modelling and simulation is one useful way to study complex slum dynamics, and knowledge derived through such tools could assist planners and policy makers in decision-making processes. In slum management, there is often a trade-off between enhancing one system at the expense of other areas: the construction of low cost housing may lead to congestion in the local public transportation infrastructure or may put additional stress on the water infrastructure. Data driven simulation allows policy makers to predict the non-linear effects across urban systems from migration, transportation to housing. Simulations are an important link between system and experimentation: without involving real-world experiments, planners can envisage the consequences of interventions across various policy scenarios and have thus become an crucial instrument for urban planners. The paradigm shift to a bottom-up modelling approach captures the interaction and processes at a finer scale. Agent-based Modelling and Simulation (ABMS) has proved useful in representing the processes underlying a particular phenomenon. The advantage of an agent based model (ABM) lies in its capacity to simulate individual behaviour and their interactions with their environment and other individuals. Geographical Information Systems (GIS) can be used to spatially represent the pattern of the phenomenon, and identify the key spatial structures which influence the micro-scale behaviour and interactions. The integration of simulation models with a GIS environment has been successfully implemented in various studies to represent spatially explicit environments in ABMS. Thus, ABMS of slum dynamics coupled with the spatial representation of the geographical region would provide a promising tool to model the growth and emergence of slums as well as the processes and patterns within slums.

Dynaslum will contribute to affordable strategic slum planning and management solutions. The model will be designed to integrate Agent-Based Modelling (ABM), Discrete Choice Modelling (DCM) and GIS within a single computational model. The model will be designed to explore links between population dynamics of slum dwellers and their residential choice behaviour to better understand the growth and emergence of slum patterns. This dissertation adopts a novel approach to understand slums as a self-organizing and self-repairing ecosystem. They evolve and adapt with extraordinary speed, and because they are comprised of thousands of people who become expert at maximizing output from scant resources, slums are highly resilient. 


\subsection{Research Goals and Key Contributions}

In this dissertation we develop a computational model, namely Dynaslum, that could function as a decision support system for city development authorities and policy-makers. Dynaslum will act as a "virtual slum" to evaluate policy interventions in a simulated environment which cannot be conducted in the actual world. Dynaslum aims to help policy makers better understand the current state of the system and its impact on future policies, but also to increase the effectiveness of slum management policies by including population dynamics and residential choice processes of slum dwellers. The key contributions of this dissertation are:

1. A multidisciplinary review and analysis of current literature on slums: We identify a set of universal factors which influence the emergence and growth of slums based on existing literature in social sciences. Based on these factors, we define an evaluation framework for computer simulation models designed to study slum dynamics. We apply this evaluation framework to literature and review the existing simulation models focusing on slum formation and growth. The review highlights the shortcomings of existing models in taking into consideration the effects of population dynamics and residential choice behaviour of slum dwellers.

2. A multivariate statistical modelling of survey data: We use Dissimilarity Index, Regression and Multiple Correspondence Analysis to conduct multivariate analysis of a unique and novel on-field survey data of 37 slums in Bangalore. We quantitatively show how spatial and social segregation reproduce, or reinforce inequality and opportunity bias within the slums of Bangalore.

3. A novel data-driven population dynamics ABM that can generate synthetic slum households: Existing models do not incorporate household life cycle (HLC) and household composition in slum growth model. However, the literature review and the survey data indicates that changes in HLC is a crucial trigger for residential mobility among slum dwellers. HLC has been extensively used as a basis of consumer behaviour and market research. Wilkes (1995) proposes a 15-stage hybrid [16] of Wells and Gubar's (1966) and Gilly and Enis's (1982) typologies [17]. In life-cycle analysis, households are classified into various life stages based on a priori definitions that reflects the typical compositions and evolutions of households in the context of a given population. In Dynaslum, HLC is operationalized and discussed extensively in chapter 6 . The population dynamics 
model in Dynaslum takes into consideration the importance of HLC in modelling slum growth and demonstrates the key differences in HLC between slum and non-slum urban households. In particular we seek answers to the following research questions: i) what are the key differences in the dynamics of a slum and a non-slum urban household? ii) what are the key factors that drives the difference in the HLC of a slum and a non-slum urban household? iii) how the difference in HLC contributes to the growth and emergence of slums?

4. A novel data-driven residential choice ABM based on DCM and GIS to understand residential mobility and inter-slum migration: One of the key outcomes of the literature review is the lack of understanding of residential mobility of slum dwellers in existing slum growth models. The residential choice ABM in Dynaslum integrates economic, social and environmental factors in choice modelling; and has the potential to bridge the gap between evidence and policy interventions for slum management. We estimate a DCM based on bid-rent theory using latent class to parametrize the residential choice model with a robust data driven socio-economic theory. In particular we seek answers to the following research questions: i) how do slum dwellers make residential choices? iii) how do social networks facilitate and influence residential location choices in slums of Bangalore? iii) how does the current socio-economic situation impacts the mobility of slum dwellers?

\subsection{Dissertation Structure}

The conceptualization and design of Dynaslum involved three different development stages in sequence: i) review of slum and modelling literature, ii) empirical analysis of slums in the city of Bangalore, and iii) design and development of the model. The research objectives defined in the previous section served as a guideline for narrowing the focus of each of the development stage. The review process is further divided into two groups and discussed in the following two chapters in this dissertation: i) review of the definition and characteristic of slums and existing slum policies and ii) review of slum growth models.

In chapter 2 we present the background of slums and review existing slum policies. The review in chapter 2 describes common characteristics of slums and important underlying factors that influence the growth and emergence of slums. In this chapter we also evaluate various slum policies and programs to identify the processes that influenced the outcome of previous policies. 
The findings provide a general background and context of slums in developing countries that is useful for the conceptualization of Dynaslum. In chapter 3 we discuss various computer simulation models that have been applied to understand slum dynamics. Here, we derive a taxonomy of key growth factors of slums which was useful to characterise data, identify proxies for missing data, steer directed data collection, and model building. The taxonomy resulted in a set of standardized evaluation criteria which was applied to existing slum growth models. Finally, the chapter presents a detailed review of existing simulation models using the standardized evaluation criteria and identified key open research questions.

The second stage in the development of Dynaslum involved experiential analysis of the slums in the city of Bangalore and is presented in chapter 4. The analysis is based on an on-field novel survey of 37 slums in Bangalore, which was further used to derive agent rules, calibrate the parameters of the model and validate the output of Dynaslum. In chapter 5 we further apply multivariate statistical techniques on the on-field survey data from 37 slums in Bangalore to quantitatively demonstrate Tilly's theory of group segregation. We find high level of spatial segregation based on religion which further leads to "opportunity hoarding" and marginalization of the minority groups in the slums of Bangalore.

The final and the most important part of this dissertation; the development of the model is presented as two separate chapters in this dissertation: i) population dynamics model, and ii) residential choice model. In chapter 6 we outline the design, implementation and validation of the population dynamics model. The idea is to take observed relations based on census data of India, combine them with demographic projections to build a model that simulates the population of slum dwellers in the city of Bangalore, India. In this chapter we demonstrate that the impacts of various demographic processes on household life cycle (HLC) can be readily investigated using a computational approach. Further, we find that slum households exhibit a large variation in the HLC compared to non-slum households, primarily driven by higher homeleaving and independent living after couple formation. An increase in smaller slum household numbers and resultant higher per capita resource consumption in smaller household challenges current urban planning practices in fast-growing cities of developing countries. In this chapter we further discuss the policy implication of our findings on the design of low-cost urban housing and positive youth development opportunities.

In chapter 7 we describe a novel agent-based residential choice model to understand the residential mobility of slum dwellers. We use discrete choice modelling to provide an empirical foundation to the model and derive behaviour rules of the agents. Residential choice component 


\section{INTRODUCTION}

implements the individual location choices to explain the inter slum migration of slum dwellers within the city of Bangalore based on social, economic and environmental satisfaction. The residential choice component is calibrated and validated using a novel field data from 37 slums in Bangalore. In this chapter we find that social and economic satisfaction takes precedence over environmental satisfaction. The residential choice model demonstrates the stabilising and

depopulating effects of social network in slums. We find that the principal disruptive effect of resettlement due to employment on slums is channelled through a nucleation process following the splitting up of slum into different parts. Finally, in chapter 8 we present a discussion of the policy implications of our finding and conclude with planned future work.

\subsection{Scope and Limitations}

Dynaslum uses a methodology that is empirical and stochastic; where the focus is on the design and validation of a new computational model which incorporates the population dynamics and the residential choice behaviour of slum dwellers. The validation is performed using the data from the city of Bangalore, India. Nevertheless, the development is clearly outlined in this dissertation so that Dynaslum could be easily parametrized and implemented for other cities in less developed countries. 


\section{Chapter 2}

\section{Slums Definitions and Characteristics}

In this chapter we provide the formal definitions of slum and how these definitions vary across different agencies. Slum literature was reviewed to determine the factors that dictate the growth and emergence of slums. Specifically, the review identified important attributes of slums in developing countries and the various growth factors which provide key processes that must be included in Dynaslum. The structure of this chapter is as follows. Section 2.1 presents various definitions of slums with a specific focus on India. In section 2.2 we list the important characteristics of slum settlements and their relevance to the design of Dynaslum. Section 2.3 identifies social, cultural and economic factors that dictates the growth and emergence of slums. Finally, section 2.4 ends with a discussion of the important processes that are relevant for the design of Dynaslum.

\subsection{Definition of Slums}

Slums are amongst the "least well understood places on earth", primarily because "they are among the most complex places on earth" with non-linear dynamics [18]. However, defining a slum is crucial for local governments and policy makers to implement targeted policies and provide access to public distribution system. The definition of slums differs from one city to another within a country and often depends on different parameters. To complicate matter further, apart from slums, there are several other terms (such as informal, sub-standard, spontaneous or squatter) associated with poor housing conditions based on the region, origin and nature of the settlement and the neighbourhood. In this dissertation we use the phrase slum 


\section{SLUMS DEFINITIONS AND CHARACTERISTICS}

and do not distinguish among the above four terminology. In the next sections we will discuss few key definitions from different organizations across the world with a specific focus on India.

\subsubsection{Definition by The Cities Alliance}

Cities Alliance, an organization which comprises of global partnership for urban poverty reduction and the promotion of the role of cities and governments in sustainable development offers the following general definition of a slum.

"Slums are neglected parts of cities where housing and living conditions are appallingly poor. Slums range from high density, squalid central city tenements to spontaneous squatter settlements without legal recognition or rights, sprawling at the edge of cities. Some are more than fifty years old, some are land invasions just under way. Slums have various names, Favelas, Kampungs, Bidonvilles, Tugurios, yet share the same miserable living conditions" [18].

The definition highlights that living conditions and security of tenure are the key parameters for identifying slums by city governments. The definition explicitly considers the following two parameters that are crucial for categorizing slums: the relative spatial position of slums in a city (e.g. core, periphery or intermediate), and the growth of a slum over time (for e.g. "some are fifty years old, some are land invasions just underway").

\subsubsection{Registrar General and Census Commissioner of India}

Most developing formulate their own definitions that are context-specific and are primarily used for the purpose of public distribution system and conducting a census. The Pranob Sen committee "set up by the ministry of housing and urban poverty alleviation to study the define slums and to estimate urban slum population for the whole country, has formulated a new definition for slums in India and has projected 93.06 million people living in slums by 2011, up by over 18 million in the last 10 years. The committee has defined a slum as a compact settlement of at least 20 households with a collection of poorly built tenements, mostly of temporary nature, crowded together usually with inadequate sanitary and drinking water facilities in unhygienic conditions" [1].

According to 2001 Census of India, "slum areas were defined as specified areas in a town or city notified as slum' by state/local government administration under any Act, including a Slum Act', or all areas recognised as slum' by state/local government which may have been formally notified as slum under any Act. The earlier definition also counted slums as a compact area of at least 300 population or about 60-70 households of poorly built congested tenements in 
unhygienic environment usually with inadequate infrastructure and lacking in proper sanitary and drinking water facilities". The new definition identifies built-up density (e.g. "compact area", "congested tenements") and access to public distribution system for urban poor (e.g. "poorly built", "inadequate infrastructure") as important criterion for slum declaration.

\subsubsection{Definition by The Karnataka Slum Areas Act}

The definition of slum in India varies across different states or different local agencies within the same city. As per the Karnataka Slum Areas Act (1973), a slum is defined as:

"Any area is or is likely to be a source of danger to health, safety or convenience of the public of that area or of its neighbourhood, by reason of the area being low-lying, insanitary, squalid, over-crowded or otherwise; or (b) the buildings in any area, used or intended to be used for human habitation are - (i) in any respects, unfit for human habitation; or (ii) by reason of dilapidation, overcrowding, faulty arrangement and design of such buildings, narrowness or faulty arrangement of streets, lack of ventilation, light or sanitation facilities, or any combination of these factors, detrimental to safety, health or morals, it may, by notification, declare such area to be a slum area."

This definition relies on the physical attributes of the settlement, population density, basic services and infrastructure of the area. As per the survey conducted by Karnataka Slum Clearance Board (KSCB) in 2010-11, there are 2722 slum areas in the State. Out of which 542 Slum areas are in Bangalore City. It is estimated that the population living in slums in the State is about 4 million, which equates to $22.56 \%$ of the State's urban population. Totally 2391 slums are declared in the state and 310 slums are declared in Bangalore City under the Slum Development Board Act (I\&C) 1973. Since, Dynaslum is developed on data from Bangalore, Karnataka. it implicitly applies this definition, which allows a context specific analysis of the slums in Bangalore.

\subsubsection{Definition by UN Habitat}

The UN General Assembly authorised UN Habitat to encourage sustainable cities with the objective of providing access to inclusive, sustainable and adequate housing for different sections of the society, including the urban poor. Therefore, it may be meaningful to analyse the parameters that are included in the definition of a slum by UN habitat. The most recent definition is as follows: 


\section{SLUMS DEFINITIONS AND CHARACTERISTICS}

"UN HABITAT defines a slum household as a group of individuals living under the same roof in an urban area who lack one or more of the following: i) Durable housing of a permanent nature that protects against extreme climate conditions. ii) Sufficient living space which means not more than three people sharing the same room. iii) Easy access to safe water in sufficient amounts at an affordable price. iv) Access to adequate sanitation in the form of a private or public toilet shared by a reasonable number of people. v) Security of tenure that prevents forced evictions."

This definition perceives that not all slums are similar and not all slum households experience similar level of hardship. The lack of one, or more, of the above basic living conditions results in a "slum household" modelled by several characteristics. The level of hardship depends on the scale of the five parameters included in the definition. The advantage of the UN Habitat definition is that it allows slum typologies to be derived so that context specific slum policies can be implemented. Besides, this revised definition is based on the condition of individual households rather than the neighbourhood. In most developing countries, the above five parameters are collected as a part of nationwide census. Therefore, this definition can be easily applied on a census data to objectively measure slum incidence in a city and the level of deprivation in each slum.

\subsubsection{Impact of Multiple Definitions of Slum}

Various definitions across different agencies could lead to erroneous estimates of slum dwellers, which in turn could hinder the distribution of resources for welfare programs. The definition of slums still depends on subjective parameters such as narrowness, decay, overpopulation, faulty design, lack of ventilation, lack of sanitation facilities. In such a scenario, the separating line between these subjective parameters, such as 'narrow' and 'non-narrow' will be drawn differently by different agencies in different cities. Thus, applying a particular definition to evaluate slum settlements in a city can lead to different estimates by various agencies. For example, in India, there are different estimates of slum population which vary a lot. The census office released a figure of 65 million slum dwellers for 2011 while the National Sample Survey Organization (NSSO) released a report indicating that the urban slum population was just 44 million in 2012 while UN Habitat estimates 150 million slum dwellers in India. Further, In India official agencies categorise slums in two broad types: officially declared ("notified" or "recognized") slums, and a second category ("not-notified" or "not-recognized") that includes all other low-income settlements. However, the differences in conditions between these two slum 
types are vast and are not well captured in the above classification. A lot of information is lost by considering slums as a homogeneous group of settlements, only distinguished by notified and non-notified types. Therefore, creating improved coarse-grained classifications is essential for developing suitable and cost-effective policy interventions.

From a modelling perspective, varying estimates based on different parameters makes it difficult to validate longitudinal slum models. In Bangalore, multiple agencies are responsible for similar tasks e.g. the Bruhat Bengaluru Mahanagar Palike (BBMP), the Karnataka Slum Clearance Board (KSCB), and the Bangalore Development Authority (BDA) all share the responsibility for the improvement of slums; Public Works Department (PWD) build houses for economically weaker sections. Moreover, the geographical extents of each agencies are not clearly marked which leads to problems in coordination.

\subsection{Characterization of Slums}

In this section we provide a short characterization of slums and identify the key impacts of slums on human well-being, particularly on slum dwellers and the poor. As mentioned in section 2.1, there are numerous slum definitions and classification criteria. Therefore, it is crucial to understand the characteristics of slum settlements.

Slums are heterogeneous and each slum in a city suffers from varying degrees of depravity. From gender inequality to all aspects of health and quality of life, living in a slum significantly impacts upon many lives. Most of the urban problems are often attributed to rural-spills into the urban slums. However, slums also acts as hubs of opportunity and rising income for the rural-poor [19]. We have identified the following key impacts and concerns of slums on human well-being.

\subsubsection{Entry-points of the poor}

According to the 'Urban India 2011- Evidence' report, 22 million people have migrated from rural to urban areas in India since 2001 [20]. In most Asian countries, the rural to urban migration continues to grow despite the poor conditions in slums. Migration of millions indicates that the rural poor perceive slums as a progression. The poor can enter cities through existing or new slums. Despite being illegal, it is widely accepted by politicians as a legitimate form of entry. The pervasiveness of dominant caste system in India leads to forward castes acting like feudal rulers in many rural areas which contributes to the migration of backward castes to the 


\section{SLUMS DEFINITIONS AND CHARACTERISTICS}

cities [21]. Like in India, other South Asian and South East Asian countries are also experiencing high rural to urban migration. The creation of remunerative wage work in urban areas also contributes to the high volume of spatial migration from rural to urban areas [22, 23, 24].

\subsubsection{Safety concerns of Women}

The safety of women is one of the key concerns in slums. Violence against women in slums is a recurrent issue. Sanitation facilities are poor or non-existent in slums. The toilets are usually shared by several households and lack privacy. Women are also subjected to domestic violence in slums. For instance, in Accra, high incidents of rape have been reported in slums. In Mumbai, women are scared to venture out of their dwellings at night. In Nairobi, domestic violence is a serious concern in slums [25].

\subsubsection{Health and Child Mortality}

Hazardous conditions and lack of access to basic services such as water, sanitation and closed sewage systems in slums lead to the spread of deadly diseases. Illnesses like malaria, cholera, and diarrhoea are prevalent in many slums. The HIV infection rate in Kibera is twice the national average. Also, child mortality is high in slums. According to "Homeless International" slum children are more likely to suffer from pneumonia, diarrhoea, malaria, or measles [26]. Diarrhoea kills 1.5 million children under five each year worldwide.

In poor communities, the use of bio-fuel coupled with incomplete combustion can cause chronic obstructive pulmonary diseases and acute respiratory infections. Respiratory infections are the most common cause of death among slum children younger than 5 years of age in developing countries like India.

\subsubsection{Lack of Education}

Children living in slums are often denied the opportunity to go to school. They are forced to work as cheap labour and they are denied the basic right to education, leisure, and play. Female literacy rates in Dar es Salaam slums are 50\% compared to an overall rate of $94 \%$. In India, slum literacy in some of the states like Delhi, Jammu and Kashmir, Uttar Pradesh, Rajasthan, Gujarat, and Goa are far below the national average [1]. Consequently, the lack of education leads to a gap in skill sets that are required to secure permanent jobs in the formal sector [27]. Disparity in literacy between slum and non-slum areas is a key indicator of slum conditions in a city. 


\subsubsection{Exclusion from formal financial system}

The banking system often excludes the poor, either because banks are underdeveloped in developing countries, or because the poor are treated as "unbankable". As a result, the people living in slums depend on loan sharks to provide financial assistance. The loan sharks demand high interest rates and further impoverish the poor. Without a steady income and lack of financial assistance, slum dwellers are unable to invest in improving their livelihood, making it very difficult to move out of poverty. In India, barely $50 \%$ of the slum households have formal banking services [1].

The lack of formal banking and financial facilities among the urban poor compels them to approach informal loan sharks who charge exorbitant amount of interests. Distribution of benefits and subsidies to urban poor are also not efficient due to the lack of formal banking services among the poor [27].

\subsubsection{Political and Social Exclusion}

Slum dwellers are stateless and are often not recognized by governments, excluding them from development policies, voting and fundamental rights. Rural-urban migration is one of the main driving forces of growing city slums. As a result, slum communities consist of different age groups, religions, origins and cultures. Inter-ethnic tensions are amplified in slums and sociospatial differentiation may take place within slums [28]. The systematic social and political marginalization of the poor slum dwellers results in further exploitation. Slum settlements are rarely upgraded as the slum dwellers are scared of the impending demolition. In 2005, around 4000 households in the Phulbari slum settlement in Bangladesh were evicted within 3 hours. The eviction led to economic upheaval and homelessness for many slum dwellers [29].

\subsection{Identifying Growth factors of Slums}

In this section we identify and elaborate the key factors which influence the emergence and growth of slums. The emergence and growth of slums can be best analysed by understanding why, where, and how slum development takes place and can be attributed to the following key factors. The following factors have been derived from various literature on slums [5, 27, 30, 9, e.g]. 


\section{SLUMS DEFINITIONS AND CHARACTERISTICS}

\subsubsection{Population Dynamics}

Rapid population growth can amplify most of the urban problems. Slowing the population growth can reduce pressure on ecosystems and natural resources, improve land and water management, and achieve a more equitable distribution of energy in urban areas. Policies pertaining to human population control will be crucial in shaping the future of our cities. Population control is usually achieved by altering the rate of reproduction or by controlling spatial mobility. Population change is the key indicator of the population growth and often formulated as:

Population Change $=\left(\mathrm{CBR}^{1}+\right.$ ImmigrationRate $)-\left(\mathrm{CDR}^{2}+\right.$ EmigrationRate $)$

The birth rate itself is a function of various local factors such as culture, birth control, economic value of children, age of marriage, urbanization, education, epidemics, family planning, and infant mortality rate. Immigration should incorporate all the aspects of migration, such as regional affiliations, religion affiliations, rural-urban migration, and urban-urban migrations.

The death rate is impacted by nutrition (quality and quantity), ageing, health care and disease control. It is well recognized that models of population dynamics such as Malthusian growth do not satisfy the observed reality and are not applicable to many real world situations. Naturally, more realistic models of populations should take discontinuous characteristics and account for individual behaviour, such as impulsive effects due to migration, impacts of cultural beliefs etc.

\subsubsection{Economic Growth}

The Gross Domestic Product (GDP) is a key indicator of economic growth. Most cities in Asian developing countries have higher GDP per capita than their national incomes. There has been a constant effort to slow down or redirect migration from the largest cities to the smaller urban areas. Such strategies have failed and led to the expansion of smaller cities in the developing countries [31]. Economic development is often concentrated in few large cities. Better job opportunities and higher quality city-life [e.g. health care, education etc.] results in a population boom in a short period, that puts pressure on the infrastructure of the city. One of the extreme discernible consequence of the rapid urbanization has therefore been the

\footnotetext{
${ }^{1}$ Crude Birth Rate (CBR) - annual number of births per 1000 people in a given area.

${ }^{2}$ Crude Death Rate (CDR) - annual number of deaths per 1000 people in a given area.
} 
growth and emergence of informal settlements as the economy is not always strong to provide economic opportunities for all migrants, and thus contributing to the growth and emergence of slum areas. Apart from the GDP per capita, the macroeconomic environment of a country can be measured by annual growth in GDP per capita, financial depth, inequality in the distribution of income, and the external debt situation [30].

\subsubsection{Housing Market Dynamics}

Housing Market Dynamics directly impacts the growth of slums as it affects the affordability of the real estate. From a slum perspective both informal and formal housing markets are important. The informal housing market impacts the slum intrinsically while the formal housing market dynamic has an external influence on slums. In India, urban land prices have increased exponentially which renders them unaffordable by the urban poor. The rapid increase in land prices can be attributed to a) surge in housing demand led by growth of housing credit; b) constrained supply of land; and $c$ ) polarized urban land markets [32]. High land values and construction costs and neglect of the housing sector is contributing to the growth of slums as the lower income group is forced to find cheap accommodation in the form of informal settlements. Hence, it is important to understand the interaction among supply, demand, and polarization of urban land markets.

\subsubsection{Informal Economy}

The global informal economy is estimated to be worth 10 trillion USD. It employs around 80 percent of the workforce in the developing countries and is the fastest growing part of the economy [33]. Workers in the informal sector lack access to basic job security and benefits. Local informal businesses have less growth opportunity and employees are trapped in menial jobs. However, the informal sector allows a vast majority of slum dwellers to avoid extreme poverty and earn an income that is essential for meeting their basic needs. The informal sector can trigger a vicious cycle from the viewpoint of a government as the authorities are unable to collect taxes which hinder its ability to finance public services. Nevertheless, some governments treat informality as a blessing in disguise, absorbing excess labour and mitigating unemployment issues [34]. It is essential to recognize the correlation between formal and informal sectors in the economy in any discussion of slums. UN-HABITAT analyses that the majority of young people employed in the urban informal sector live in slum areas. Hence, a thriving informal economy leads to a stable slum and more people would be attracted to join the slum [5]. 


\section{SLUMS DEFINITIONS AND CHARACTERISTICS}

\subsubsection{Local Topography}

Slums or Informal settlements (IS) can be characterised by their site and situation. Usually, slums are situated at sites, considered unsuitable for formal real estate such as near railway tracks, banks of rivers, dumping grounds, abandoned or unused land, near industrial areas and in wetlands. They are also found in what might be considered 'unattractive' areas such as cemeteries, and close to waste disposal sites [35]. Overall, slums flourish on any marginal or vacant urban lands. Natural hazard-prone areas of the city can also be correlated with the existence of slums. These risk factors include building collapse, fire, disease spread and flooding. While older slums are found in central city areas, new slums are more likely to develop in the peri-urban areas. Informal dwellers who are not newcomers, tend to have previously lived in slums (probably nearby) dominated by people from a similar ethnic, cultural or religious background. Similarly, new migrants to urban areas are more likely to settle in neighbourhoods with strong cultural, ethnic and religious ties.

\subsubsection{Street Pattern}

The genetic code of a settlement gets imprinted in its street pattern. They emerge as walking paths connecting markets, residences, and nodes of transport hubs. As they evolve, accommodating local transport, street shops, meeting points, recreational area, they follow the needs of the residents directly. The street scape inside slums determines their shape and direction of growth, as slums tend to expand along attractive streets like those hosting street markets, economic centres etc. Slums are also constrained by infrastructural urban barriers such as a railway track on one side of the settlement, which restricts the growth of the settlement towards that side. Street patterns inside slums evolve to achieve optimality as they grow and is primarily influenced by places of interest [e.g. temples, economic centres etc.]. Streets and paths in slums follow a hierarchy of different widths, finishes with broader roads serving smaller roads. The ratio of private territory to that of public territory is quite high. The close packing of dwellings reduces the external surfaces exposed to sun and results in maximum shading of private and open spaces. In many cases, narrow lanes permit double access to the dwellings at the front and back of the house, which would be useful for dwellings that combine residence and commercial activities [36]. Such a set-up accommodates extended family by giving them a separate access. In case of insufficient land, such a solution provides an affordable means to accommodate more people. UN-HABITAT advocates for a move towards the opening of streets in slums as a strategic intervention for citywide slum upgrading [10]. 


\subsubsection{The politics of Slums}

The local politics plays a massive role in the growth of slums in cities. The local government directly impacts the legal and regulatory affairs. A lack of political will can be catastrophic to the cause of slum resettlement. In India, local slum leaders are connected to political parties and they let slums grow as vote banks [37]. In Mumbai, vote bank considerations have prevented the eviction of illegal squatters around the airport, thus preventing the utilisation of the land for the airport's expansion. Eviction is carried out selectively and is largely governed by micropolitics of the region [38]. In Jail ward, India, for instance, there are three slums - Takia, Saraswati Nagar and Fakirawadi and these areas contribute a huge chunk of voters. Besides, in Jail ward, past trends indicate that as compared to $47 \%$ polling in residential areas, the figure was between 65-90\% in slums. During the February 2012 elections, of the over 2 million registered voters in Nagpur, 0.5 million are from slums [37].

Poor people know that they have power during elections, but bribing and intimidations also play a role. Loyalty to the local slum leader is important, as he is also the one responsible for protection. Official recognition of a slum, meaning access to public services, can be at stake during election time. Government jobs, such as public sweepers and municipal trash collectors, are also granted during election time $[39,30]$.

\subsection{Discussion}

In this chapter we have identified that substantial quantitative analysis on population dynamics and residential choice behaviour are well documented for urban households. However, lack of similar quality quantitative data from slums in developing countries is evident. The lack of accurate data is partly due to the multiplicity of definitions and agencies that are involved in the process of slum notification and development. However, in recent years with a renewed focus on poverty alleviation in most developing countries, we now have a better understanding of the spatial feature of the slums, population densities and socio economic conditions of slum dwellers. The literature delivers a foundation for reviewing existing slum growth model and developing a framework for directed data collection that can provide multidimensional nonlinear understanding of slum dynamics. The literature could lead to formulation of rules for simulating individual household behaviour and identify important processes and growth factors.

Further, in this chapter we have shown that slum policies in developing countries have been implemented without analysing their negative impact a-priori. The urban poor have 


\section{SLUMS DEFINITIONS AND CHARACTERISTICS}

largely been unaffected by any policy interventions which are usually characterized by corruption and leakages. One major problem is the lack of robust exploratory and diagnostic tools that can evaluate planned policy interventions, with a few exceptions discussed in further detail in chapter 3. Dynaslum is designed to act as a "virtual slum" so that urban planners can assess and evaluate planned slum management policy intervention in a simulated environment prior to their implementation in the society. 


\section{Chapter 3}

\section{Review of slum models}

The purpose of this chapter is to formulate evaluation criteria for the assessment of existing simulation models and to conduct a literature review of informal settlement (IS) simulation models [4]. Previous slum studies, providing a relevant body of knowledge for the formulation of evaluation criteria, differ in their scope [e.g. develop targeting strategies, better understand slum areas, or identify slum areas], research methodologies employed, and geographical scale of analysis. The five major methodological approaches that have been used in previous studies are discussed as follows. The first approach was based on philosophical ideology. Theoretical strategy was advocated to solve the problem of slums. In his book "Freedom to build" (1972), John F. C. Turner argued that governments should only provide the basic amenities such as good sewage, clean water, and good roads [40]. People would gradually improve the housing on their own. Such theories were also stated by de Soto [41, 42], but both were strongly criticized by Burgess $[43,44]$ and Gilbert [45] as they neglected the role of customary tenure and management as existing legal institution and past experiences. The second line of research focused on assessment of slum policies and programs. Retrospective studies were conducted to evaluate the slum policies. These are ex-post assessment of various slum policies (e.g. selfhelp policies were evaluated by Abelson [46]; Harris [47]; slum re-development policies were evaluated by Mukhija [48]). The third approach uses qualitative and quantitative methods. It provides an elaborate analysis of urban poverty and slum growth and is independent from other policy discourses [6]. These studies can be categorized under case study approach as it provides a detailed summary of a specific slum but lacks the generality [49, e.g.]. Similarly, a statistical approach using slum indicators elaborates a general conclusion but continues to be limited in their effort [50, 51, e.g.]. The fourth approach emphasizes advances within the fields 


\section{REVIEW OF SLUM MODELS}

of remote sensing (RS) and geographic information science (GIS), which allows one to map, compute and spatially analyse slums $[52,53,54]$. High resolution remote sensing methods for mapping slums are being used more often [55, 56, 57, 58, 59]. However, traditional GIS and RS analysis address dynamics by comparing independent static representations (GIS maps or RS images) of two different moments in time, while in simulation models changes in the state are determined by the original state as well as changes in the input data. The fifth approach is based on formative or action research. BRAC development institute (BDI) has conducted action research in Bangladesh. The aim of the research is to address poverty through research, action, learning, partnership and participation. In that particular case, it analysed the current conditions, climate change impacts, and community mobilization strategies. However, similar researches have been conducted around the world [60, 61, e.g.].

Recently, computer simulation models have been used to study urban phenomena. Urban researchers have applied cellular automata $(\mathrm{CA})$ to investigate various situations such as land use dynamics [62, 63, 64], large-scale regional development [65], social segregation in urban space [66], gentrification [67], and urban growth and sprawl [68]. In all these instances, GIS played an important role in manipulating real world data. Although some of these applications highlighted the pedagogical and research contribution of cell-based models in geographical sciences [69], there has been an increasing research investment in developing cell-based models as prescriptive and useful tools for real world situations [70]. There is also a growing amount of work in the field of $\mathrm{ABM}$ and urbanization [71, 72, 73, e.g.]. However, the use of ABM to describe and understand slum formation is still rather new. In general, ABMs incorporate the behaviour of individual agents based on their interactions with other agents and the environment. This can be used to understand the expansion of global phenomena such as slums. The six models discussed further in this chapter relate to the existing informal settlement (IS) computer simulation models and represent a comprehensive set of simulation model types.

\subsection{Developing a framework for evaluating slum models}

The US Environment Protection Agency (EPA) review of urban models is a useful starting point in selecting the appropriate evaluation criteria for urban and IS dynamics in developing countries [74]. The EPA's main finding was that urban land use models should be selected on the basis of their ability to predict emergent patterns. That is, models should be able to predict new developments using relevant variables with logical rules or conditions of change. Whilst 
the EPA's check-list was useful, it was felt that specific selection criteria needed to be added to fit the context of slums in developing countries as the EPA's criteria had been developed for planning agencies in the USA. In this study, we develop a set of criteria to evaluate urban models designed to simulate urban and slum growth in developing countries [75]. The evaluation can be classified in three categories namely: Level Of Detail, Emergence and Growth Factors, and Robustness and Implementation. Level of Detail deals with the spatial resolution, temporal resolution, and Level of Abstraction. The growth factors have already been listed and discussed in the previous chapter. The category Robustness and Implementation deals with Sensitivity, Calibration, and Data Source of the models. The categories are explained as follows:

\subsubsection{Spatial Resolution}

Spatial resolution results from the division of a study area into regular entities such as grid cells, and is determined by the number of individual units in relation to the spatial extent of the entire study area. Urban phenomena, for instance the residential composition, vary in space and time, depending on the nature of the respective phenomenon. Accordingly, it is essential to consider a model's ability to handle the spatial resolution, that is to say whether the model is able to represent specific processes and states in a spatially disaggregated manner. It is critical that a model simulating urban dynamics employs a spatial resolution that fits the context of urban areas in developing countries, and also the size of the urban region in consideration. A city needs to be examined with respect to its location in the region and the influence of the peri-urban region on the city needs to be included in the analysis. Cities and regions share a mutually reinforcing relationship and the increasing use of the term "city-region" highlights the significance of the relationship. The spatial resolution embedded into a model can vary from a few meters to several kilometres and it is critical that the design of the model considers the spatial resolution of the data available in a certain geographic context.

\subsubsection{Temporal Resolution}

One of the EPA criteria is the ability of a model to handle the temporal resolution. According to that framework the temporal resolution can vary from a few minutes to several years depending on the purpose of the model and can have continuous multiple time steps or lag time periods. However, a model that can be adapted to the urban and IS in developing should have a temporal resolution of 1 to 5 years, because this is considered as a reasonable period where changes can 


\section{REVIEW OF SLUM MODELS}

be noticed [75]. It is also important that a model allows for continuous and multiple time steps because IS expand continuously and could follow different stages.

\subsubsection{Level of Abstraction}

Level of abstraction refers to the processes and states a model represents, which is closely related to the temporal and spatial properties of input files required to run the model. This criterion is important because, in the context of urban areas in developing countries, availability and reliability of suitable input data (type, size and format) for a given model is a major challenge and cannot always produce reasonable outcomes. For instance, input data with a small cell size $\left(\mathrm{m}^{2}\right)$ or too coarse $\left(\mathrm{km}^{2}\right)$ would not be relevant to Yaound $\tilde{\mathrm{A}} \subset$ in Cameroon [75].

\subsubsection{Growth Phenomenon}

All the factors contributing to the growth and emergence of slums (discussed in chapter 2, section 2.3) have been used to review the models. In order to present the dynamics of IS in a comprehensive way, it is also crucial to include the relevant growth factors in the model structure.

\subsubsection{Sensitivity Analysis}

Sensitivity analysis tests the sensitivity of the model output with respect to changes in the input parameters, model's properties and structure [76].

\subsubsection{Validation and Calibration}

Validation assesses the degree of correlation between the model output and the conceptual framework of the model. The calibration of the model drives the validation. Three categories of validation and calibration methodologies have been proposed: the "indirect calibration approach, the Werker-Brenner empirical calibration approach" [77] and the "history-friendly approach". The goal of indirect calibration approach is to establish a realistic model by understanding the empirical reality to which the theories must be fitted [78]. Like the indirect calibration approach, the Werker-Brenner approach makes use of stylized facts and empirical data, but also employs extrapolation procedures to validate that the model output matches the real data. This method needs two sets of historical data, one set to calibrate the model and the other to validate the model [79]. Finally, the history friendly approach uses explicit case studies to calibrate the parameters, derive rules for agent interactions and agent decision [79]. 
This approach makes use of empirical data to establish initial parameters and conditions. It is not the purpose of this approach to produce simulations that closely match the quantitative values observed in the historical data, but to match the overall pattern in qualitative features, particularly the trend behaviour of the phenomena simulated.

Collecting empirical data from developing countries is extremely difficult. A robust model should account for the lack of data by using proxies. As an example, the vegetation fraction may be used in conjunction with other covariates to create a more robust model for predicting slum presence, or when utilized as a proxy for neighbourhood characteristics [80].

\subsection{Taxonomy of Slum Models}

A review of the existing literature was conducted to identify informal settlement modelling resources that have the potential to simulate the emergence and growth of slums in developing countries. To date, a variety of IS dynamics models with different levels of complexity, structure, and purpose have been developed. The purpose of this section is to use the evaluation criteria formulated in section 3.1, to assess the capacity of existing IS models for exploring and simulating urban and informal settlement (IS) dynamics in developing countries. The synthesis of this analysis and review is presented in table 3.1, followed by a detailed discussion on each model. Each description also highlights the relevance of the model to be used in the exploration of slums in developing countries, as well as some of their advantages and limitations. As depicted in table 3.1, most models use annual time steps except MSGM [81]. Also, all models listed in table 3.1 have incorporated population dynamics in their model, but only Slumulation [6] captures the role of politics, informal economy, and housing market dynamics in the process of slum formation and expansion. There is no general pattern with respect to the availability and access to good data. ISGM [75] highlights the importance of accurate real world data and the high level of realistic spatial data representation to achieve a realistic model output. The main limitations of the ISGPM [82] is the unavailability of data with respect to the residential locational behaviour and spatial income distribution of the agents. The lack of data limits the ability to validate the spatial pattern of each income group against their pattern in reality.

While all models have performed a thorough sensitivity analysis, they lack uncertainty analysis for determining the reliability of model predictions due to uncertainty in model inputs and parameters. A pattern oriented modelling approach could be implemented to make models 


\section{REVIEW OF SLUM MODELS}

structurally realistic and less sensitive to parameter uncertainty. Also, the realism of patternoriented modelling helps parameters interact in ways similar to the real world and therefore it could be possible to find values of all calibration parameters that match multiple patterns [83]. We have discussed these patterns for slums in section 3.1 and have identified the patterns that are essential to build computer simulation models in the third column (Emergence and Growth Factors) of table 3.1.

\subsection{Description of existing Slum Models}

This section discusses the six computer simulation models for exploring IS in developing countries which have been identified in the literature review. Each model is described in detail along with its key functionalities. The section also highlights the pros and cons of each models. The models have been evaluated based on the evaluation criteria defined in section 3.1.

\subsubsection{Informal Settlement Growth Model (ISGM)}

The ISGM, is a cellular automata based simulation attempt by Sietchiping [75] to forecast the location and emergence of informal settlements in Yaounde, Cameroon. The ISGM is specifically aiming to:

- enrich the discourse around urban dynamics and the prediction of IS expansion in Less Developed Cities (LDC) cities;

- accompany and support urban planning and decision-making processes;

- provide a framework for discussion amongst urban stakeholders on how to handle the rapid expansion of IS;

- simulate spatio-temporal systems behaviour of IS under various conditions and scenarios;

- enable new discoveries about IS functioning by means of computer based experiments.

The spatial discretization of the model is a two-dimensional matrix of regular grid cells. Each layer in the model comprises of at least two states [e.g. slum and non-slum], which are identified using a unique identifier (e.g., green: 15, vacant land: 1). A randomly selected cell changes its state from non-slum to slum only if it has one neighbour which is a slum. This constraint limits slums from emerging in an isolated area and clusters the slum patterns. The other land uses remain static in the model. A strong assumption in the model is that the cells 


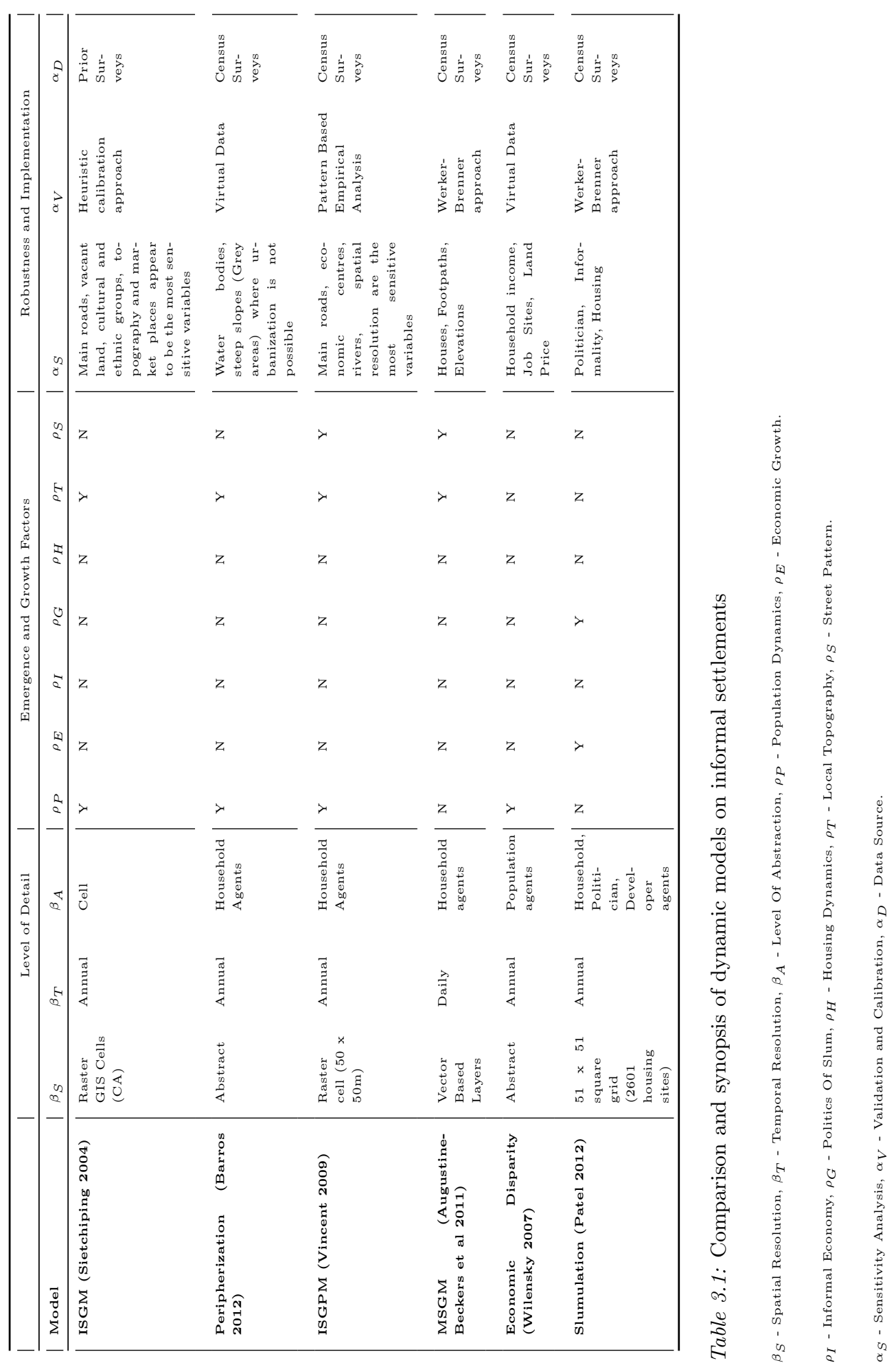




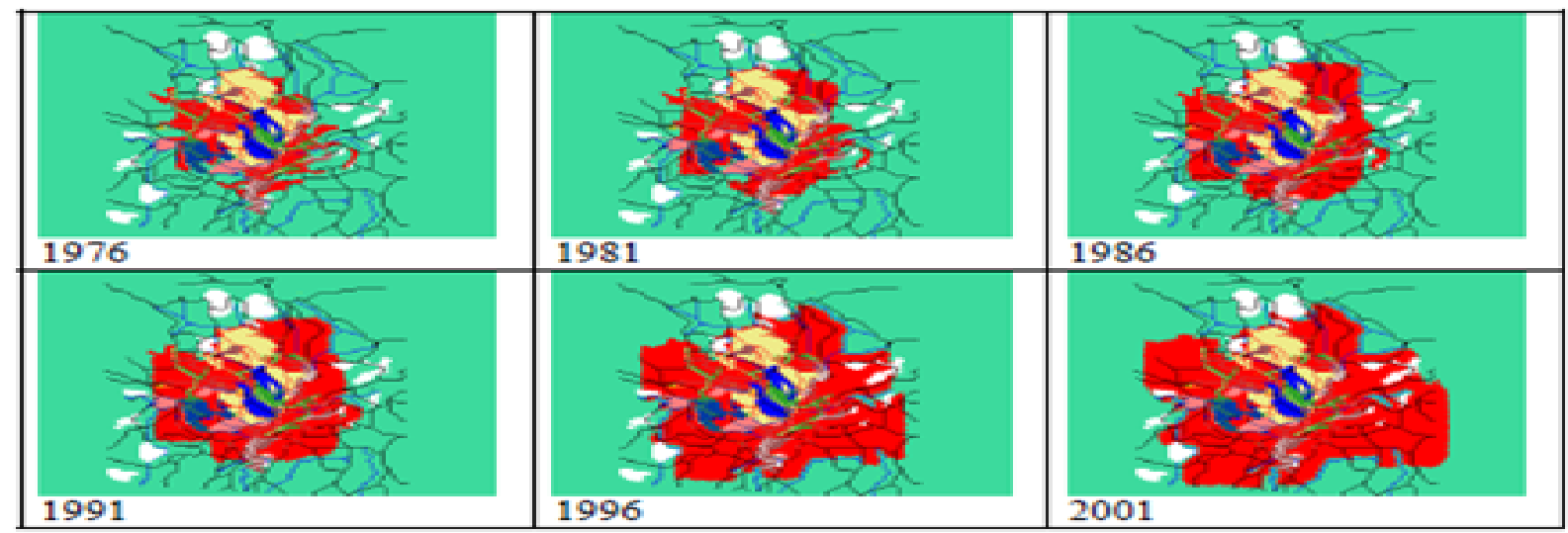

Figure 3.1: Simulation Results (Year 1976, 1981, 1986, 1991, 1996 and 2001 shown in legends) from ISGM Model. Source: Sietchiping 2004.

which are slums do not die and always maintains its current state. This basically suggests that a slum cannot be included in the formal development process of the city.

On the one hand, the probabilities for each factor or parameter reflect the relative likelihood of occurrence for single or multiple conditions. There are two levels of probability: general and local. General probability equally applies to the entire lattice, stages and parameters, whereas local probability declares the condition for a specific rule to be implemented. Each of these probabilities range from 0 (no chance) to 1 (perfect chance). If the probability is equal to 0 , then the macro proceeds to choose another random cell. If the contrary is true, the macro will then change the vacant cell to a slum cell, if the iteration quota has not yet been reached. The ISGM also implements positive and negative weights of evidence parameters, which are associated with the probability. The positive weight of evidence, or boost, refers to the attractiveness of the variable to the IS. The greater the value of the boost factor, the greater is the likelihood for the emergence of an IS cell in the neighbourhood. For instance, to test a road-driven ISGM, the probability boost of roads is increased to the maximum, whereas the weight of other factors is significantly reduced or ignored. Figure 3.1 shows a simulation output of the ISGM model.

The ISGM model relies heavily on empirical data for the calibration and validation of model. Data is derived from sources such as fieldwork, census data and existing maps. The choice of input maps, land use categories (initial and final years), water bodies, religious places and markets and cultural and ethnic places) was determined by their degree of influence on the emergence and growth of slums. All input layers have the same configuration and structure (e.g., 313 columns and 250 rows, i.e. 78250 square cells) with a cell resolution of 43 meters to 
reflect the scale of the original maps. Data was also collected from various empirical studies conducted on the city of Yaounde, in Cameroon.

The authors of the ISGM model have performed a detailed sensitivity analysis to understand the model's sensitivity to different input parameters. Each simulation output was compared to the spatial configuration of IS in the base year as well as target year. While all input parameters have been tested for sensitivity, the following parameters have been mentioned as the most sensitive parameters. The model generated a more condensed form of IS patterns when specifying land use constraints, compared to the emergence of IS near existing IS with a probability of 1 without imposing that constraint. Concerning the other mentioned parameters, the model was also sensitive to road layers, river system, gradient factor, places of worship, market place, and the contribution of cultural and ethnic composition. The ISGM uses a heuristic calibration approach, which consists of altering one parameter, variable or value at a time, and then computing the results for each parameter specification. This "trial and error" approach could be tedious and time consuming and rely on the expertise of the modeller to select the parameter values that best represents the real system.

The main weakness of this cellular automata modelling approach for simulating urban dynamics is its inability to handle top-down processes such as planning policies (e.g. zoning, planning incentives, etc) that are enforced by urban policy-maker; these are not included in CA urban models. Also, raster-based CA models cannot be used to simulate shape and sizes. The ISGM do not include human level behaviour and implements stochastic transitions between cells. Another weakness of cellular automata models is the inability of the automata cells to move within the lattice in which they reside [82]. This makes it impossible to simulate forces such as macro-scale political and socio-economic forces that influence urban expansion in the urban system. However, the cell state is binary which depicts if the land use is informal settlement or not, ignoring non-residential land usage. The ISGM model has a high dependency on historical data, which might not be available for developing countries.

\subsubsection{The Peripherization Model}

Barros $[84,85]$ implemented an ABM which describes growth of slum based on households' income level. The model studies how individual decision making can be included into modelling of slum dynamics. The model simulates the growth of the city by showing that low income households emerge at the periphery of the city and with time are absorbed by the city. These 


\section{REVIEW OF SLUM MODELS}

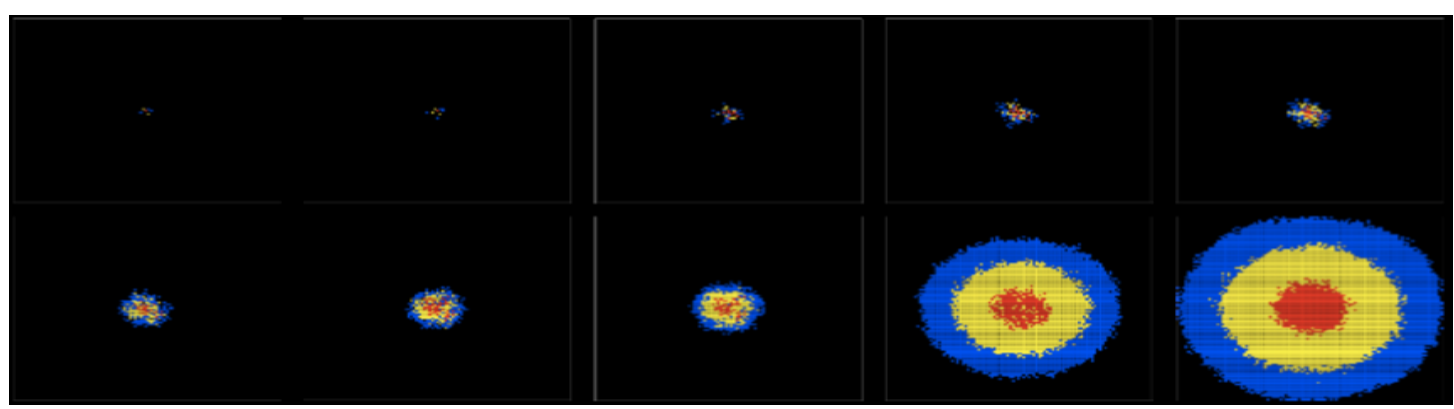

Figure 3.2: Simulation Results from Peripherization Model. Source: Barros 2012.

low-income neighbourhoods are in turn displaced by high income households further towards the periphery of the new city boundary.

The model simulates how the residential decision making of the people influences urban growth in Latin American cities. All the agents have the same location preference, they all want to settle close to the city centre which is well serviced with social and infrastructural amenities. However, locating close to the city centre is restricted by the economic power of the agents and how far they are willing to travel in search for an ideal location. The high income agents can locate anywhere in the area except for areas occupied by agents of the same income group. The middle income agents can locate anywhere except for areas where a high-income or an agent of the same income group is settled, the low-income agents can locate only on vacant lands. The model's third module deals with inner city decay and the transition in the income status of agents [85]. Agents settle in a place based on neighbourhood density and closeness to agents of the same income class. Age and density variables are also introduced into the model to simulate the housing transition of agents from higher to lower income group and from lower to higher income group. Spatial constraint such as water and topography are introduced into the model. The cells that hold the various spatial constraints are defined as exclusion cells, meaning they are restricted areas for settling. Figure 3.2 shows a simulation output of the peripherization model.

The Peripherization model uses income distribution maps of Sao Paulo, Brazil and is based on the Census dataset. Although these maps are static representations, the patterns of income distribution along with the simulation model helps to understand the locational pattern generated at the peri-urban regions in Latin American cities. Each model parameter was tested for its effect on the model behaviour. However, the outcome of the sensitivity analysis indicates that the two main parameters "number of cells that the agent walks before trying to settle in 
a place" and "proportion of agents per income group" drives the model behaviour. The results indicate that the existence of a greater percentage of high income agents leads to faster development as high-income cells promotes urban development in the model. Peripherization model is also sensitive to spatial constraints such as bodies of water, steep slopes, or unfavourable land where urbanization is not possible.

The peripherization model has some limitations as a model for simulating urban growth in Latin American cities. The main limitation of the model is that it is built in a hypothetical environment, i.e. the spatial resolution is not to scale. As a result the major driving forces behind urban growth such as roads, topographic and demographic information (income data, population data etc) were excluded from the model. It is also strictly developed for urban growth in Latin American cities, as it does not consider conditions that occur in cities of other parts of the world such as land and housing tenure systems, politics, proximity to major roads, locating in swamps, etc. The role of the environment is not well defined in the model and generalizes slum as housing for poor people [6].

\subsubsection{Informal Settlement Growth Pattern Model}

Vincent [82] designed a model known as the Informal Settlement Growth Pattern Model (ISGPM), which was based on the Peripherization model. The agents and their behaviour are based on the peripherization model described above [85]. Instead of a random walk approach, the search process implements a utility maximization approach. The behaviour of the three agent groups (high, middle and low income group) is modelled in the environment. At the start of the model, initial agents are created in proportion to the population data of the start period and the cell they occur takes their colour. At each step of the model, a group of new residents are created based on the population change in the census data for the period 1992 to 1998. Unit tests are done at different stages of the model building in the view of verifying the model. Calibration of the model will also be used in the verification process which involves checking the parameters in the model (the three income groups, selected driving forces of spatial growth, price of land, and population growth rate) to see if they are consistent with relevant description of the model. Also in this step, the model is validated against historic Census data of the study area from 1992 to 1998. The map of Ukonga ward in Dar es Salaam was used as the model environment in the implementation stage. The map consists of four layers namely, the economic layer (this consist of industrial, commercial, institutional areas, transportation), spatial constrained areas (river, flood prone areas, recreational centres, swamps, river valley, 


\section{REVIEW OF SLUM MODELS}

salt pan, and rivers), major roads, and landscape (this consist of planned residential area, informal settlements and vacant areas). The choice for a spatial resolution of 50 by 50 meters was driven by the size of the study area and size of the population [82]. A pattern based empirical analysis technique is used for validating the model, FragStats (pattern analysis software) is used for comparing the spatial patterns in the simulated output and the historic data by calculating five spatial metrics: the Large Patch Index (LPI), Edge density index (ED), Mean Nearest Neighbour Distance (MNN), Number of patches index (NP) and Percentage of Like Adjacencies index (PLADJ) of both data in different snapshots. This method is also used to compare the spatial growth pattern of informal settlement in Mbagala, Ukonga, and Kawa wards in Dar es Salaam.

In the ISGPM the utility level of each agent is a function of the land price, proximity to major roads, economic centres and rivers, and the satisfaction level of the agents. A series of sensitivity tests were conducted based on different utility level of agents of each income class to see how sensitive the model outputs are. Also simulations with different spatial resolutions of $50 \times 50$ and $100 \times 100$ resulted in different spatial patterns. The experiments indicate that the tendency to settle down quickly increases when an agent samples a higher number of locations, and the required threshold of neighbourhood density (density of agents of similar income class accepted by an agent) decreases.

As reviewed by Patel [6] "this model does not allow more than one agent in a $50 \mathrm{~m}$ x $50 \mathrm{~m}$ single cell, which is a large patch especially in a slum context." Combining the effects of all the driving forces into one indicator (proximity to major roads, economic centres and price) is one of the biggest limitations of the model. This makes it impossible to examine the effects of each of the driving forces individually on the spatial pattern produced in the model. The road layer is also static in nature and does not take into account the development of new major roads from minor ones.

Some very important factors were simply not incorporated into the model; this was because data for simulating these factors were insufficient. These factors include demographic and socio-economic factors that affects the people (agents) such as age distribution, marital status, etc. These factors are important because they show the number of people that will resettle in another location as they tend to marry at a certain age to start their family (household). They might also relocate because of their work, among other things. Another factor of great importance is the landscape, particularly the topography, river valleys, swamps, water wells and water distribution points. Further, the model simulates a municipality in isolation and 


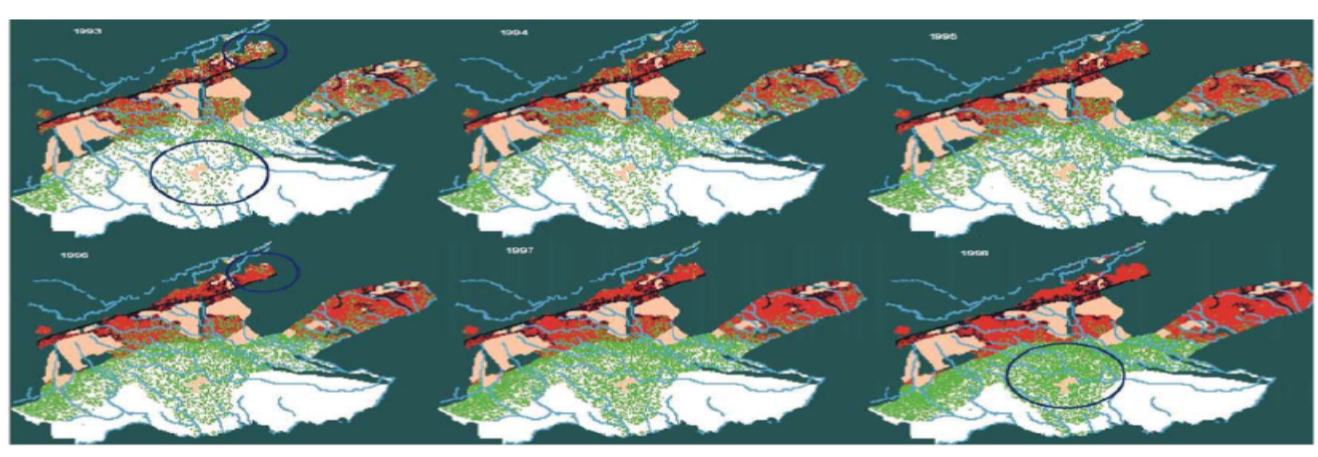

Figure 3.3: Simulation Results from ISGPM Model. Source: Vincent 2009.

ignores the interaction with other parts of the city. Figure 3.3 shows a simulation output of the ISGPM model.

\subsubsection{Manzese Settlement Growth Model}

Manzese Settlement Growth Model (MSGM) by Augustijn Beckers et al [81] attempted to model the physical growth inside a slum in Dar es Salaam, Tanzania, by building on previous models and relying on surveys. The primary focus of the model is particularly on the Manzese settlement in Tanzania using previous experimental studies for identifying important behavioural rules of the agents in MSGM. The model produces a spatial configuration of houses by implementing the following three basic rules: extension of a building, in filling among dwellings, and enlargement of existing dwellings. The model shows that by implementing the above three rules, a spatial structure of a slum could be generated that is very similar to the empirically observed structure in Mazese, Tanzania. The model consists of two types of agents: the renter and the home owner. The number of agents created in the model depends on the estimated population growth rate during the simulation time period. The agents represent a particular household and not individuals. The environment in the model consists of spatially explicit vector based layers of houses, roads and pavements, elevations and swampland which is created based on empirical data representing the conditions in Manzese settlement. The author suggests that different actors including the government influence the settlement process, but the role of government is not clear from the simulation results. The model does not have human agents driving the simulation, hence change in environment does not impact any behavioural changes in the agents. The simulated result of the model was compared to analyses on historical growth of Manzese settlement. The analyses focused on the general settlement preferences (e.g. 


\section{REVIEW OF SLUM MODELS}

alignment of houses, number of houses in flood prone areas) and method of settlement infilling and extension.

The calibration and validation of the model is based on five time points representing the actual settlement patterns in the years 1967, 1975, 1980, 1982 and 1987. For understanding the model's behaviour, the authors of the model have conducted a sensitivity analysis of 150 runs using different input parameters, divided over three time periods, 1967 to 1975, 1975 to 1980 and 1982 to 1987. The sensitivity analysis was performed for roads, footpaths, flood zones, and three different construction rules namely: infilling, extension, and enlargement. During the period 1967 to 1975 , the model output shows an average of $77 \%$ of new houses along a road suggesting a high sensitivity towards road. The results show that the attraction to build houses along a road instead of the footpath should be dependent on available space as the preference for roads decreases with less available space to build houses. The results suggest that using two rules gives more realistic patterns as compared to a single rule.

The model ignores the impact of other slums on the Manzese settlement. As the settlement grows, agents may move out of Manzese settlement into other parts of the city which is not captured in the model. The building enlargement rules are the same as the construction of new houses and is driven by the attractiveness of the location such as transport infrastructure, proximity to the road and existing houses. However, the model does not capture the impact of dwelling which are constructed to house both commercial and residential units. Also, the model focuses primarily on residential buildings in informal settlements and ignores the economic centres within such settlements. The environment created in the model is static, that is the roads and footpaths do not evolve along with the building process. However, in most slums, street patterns evolves along with the organic growth of slums. The model also imposes a restriction on agent behaviour as there is no mechanism of selling houses. In a real world scenario, home owners derive utility from their house and based on the land and housing market may decide to sell their house and become a renter. However, the model may be useful for simulating and understanding the geometry of informal settlements. Figure 3.4 shows a simulation output of the model.

\subsubsection{Economic Disparity Model}

The economic disparity model was developed by Martin Felsen and Uri Wilensky in Northwestern University, Chicago [86]. The theoretical framework of the model is the bid-rent theory 


\subsection{Description of existing Slum Models}
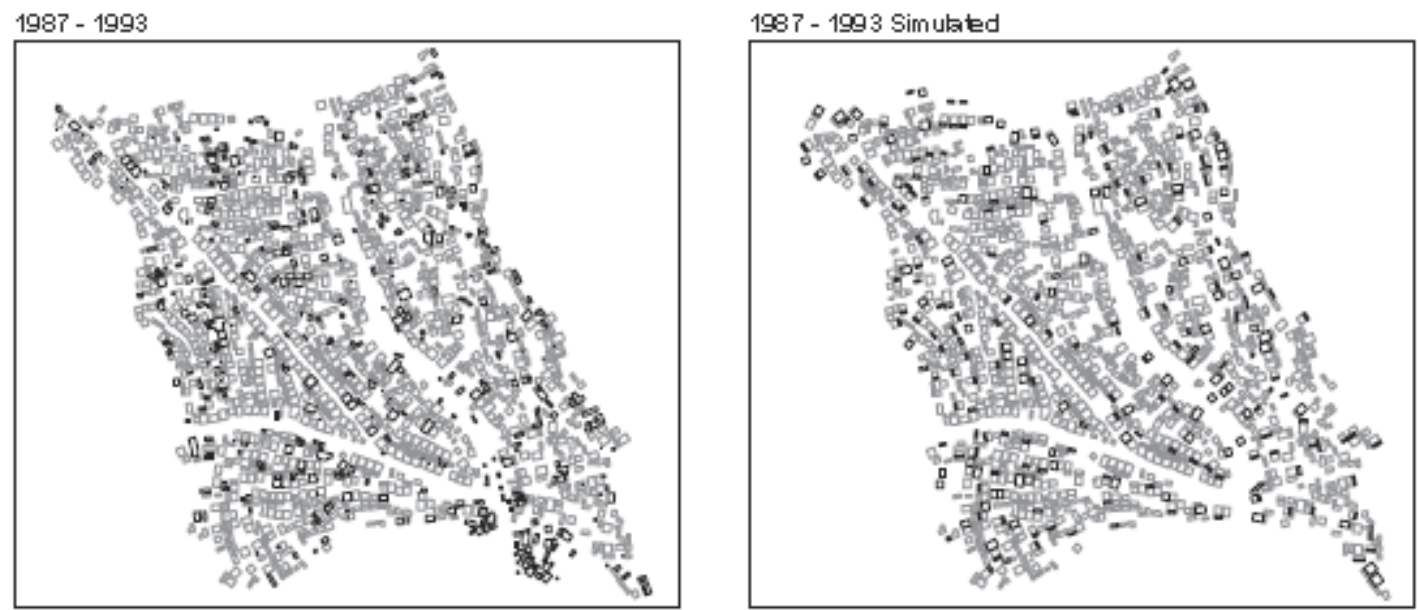

Figure 3.4: Results from 1987-1993, empirical data on left, simulated data on right. Source: Augustine-Beckers et al 2011.

which refers to how people compete with one another for land close to city centre. Prices and demand of land changes as distance towards the city centre increases.

The economic disparity model studies settlement patterns using the socio-economic status of the agents to decide their locational preference for residence. It was adapted from the bidrent model which is noticeable in developing countries where most infrastructure facilities and services are found in proximity to high income settlement areas. The model is based on two main variables: population growth and landscape of the city. It simulates the growth of two populations (rich and poor), who settle in the city based on the environmental quality, the cost of living, and access to central business district (employment centres). The two population agents have the same locational preference; they all want to locate close to city centres (employment centres), however their residential decision is restricted to their economic power to afford living in these places. There are three major structures used in building this model, they are as follows:

- Job sites: they are created and destroyed; when the land value in an area increases, a job centre is created, but when the value decreases the job site is destroyed. The people want to live close to this job sites but consider the price (cost of living) and the quality of the potential location. 


\section{REVIEW OF SLUM MODELS}

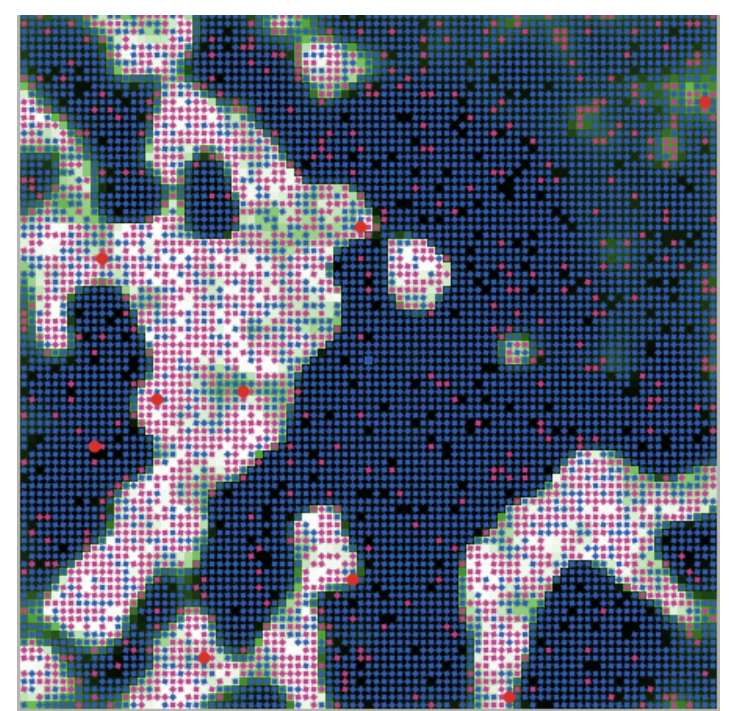

Figure 3.5: Simulation Results from Economic Disparity Model. Source: Felsen, M. and Wilensky, U. 2007.

- Location: Both agents (rich and poor) wish to locate close to a job site (employment centre) but have different priorities. The rich people seek a location that has a good quality regardless of the price, while the poor people seek locations of low price disregarding the quality of the land.

- Consolidation rules: Rich agents who relocate to an area, increase the land price, while the poor people cause the land price to decrease.

At each time-step new people (rich and poor) enter the city, but when they get old they die. As the quality and price of the cell changes, the state of cell changes, which leads to the development of different pattern. Figure 3.5 shows a simulation output of the Economic Disparity Model.

One of the problems with the above models is that they do not take account of the residential decision making of the urban people and the driving forces that influence the growth pattern of informal settlements in cities of developing countries. In order to understand the growth pattern of these IS, it is important to consider both the processes and actors that contribute to the proliferation of these informal settlements in the proposed ABM. 


\subsubsection{Slumulation}

Patel [6] developed an ABM called Slumulation, based on the city of Ahmedabad, India. Slumulation is designed to "answer three types of research questions related to slum formation and expansion, where, when and how". Slumulation integrates several modelling techniques, which includes Discrete Event Simulation (DES) and ABM. In Slumulation, the household agents make residential choices, thus adding to the existing housing demand; the real estate agents build dwellings on allowed land parcels; while the politician agents optimize benefits to get more votes from slum dwellers. The magnitude of the subsidy depends on the proportion of slum population in the ward and has been used in the model to discount the economic rent. In Slumulation, households contribute to the demand, while politicians and developers control the supply of dwellings in the simulation. However, the environment is built on two spatial scales and both politicians and developers operate at different spatial scales. The first layer simulates prospective slum sites in which dwellers make residential choices and developers make property-development decision, whereas the politician agents operates in the second layer which comprises of electoral wards. A user specifies various model parameters such as population growth rate, initial size, percentage land in the centre of city, percentage inadequate land in the core of city, economic growth rate, initial income distribution, level of informality of the economy and price diffusion rate for housing. The model is executed annually for a specified number of years and simulation outputs are captured at each time step leading to a final spatial patterns of slums which is presented as the model output. Figure 3.6 shows a simulation output of Slumulation.

The demographic and socio-economic data was collected from Census of India. The results of sensitivity analysis indicate that the slum-dwellers' are attracted to subsidies provided by the politicians and the slum population increased with a higher population growth rate. On the contrary, a higher economic growth reduced the number of slums in both the centre as well as the periphery which is not evident in the city of Bangalore, India. The slum population also increases with the increase in the informal economy.

Slumulation currently only tackles the spatial scale and does not include different temporal scale in the model. The multi scale processes in the model are not explained properly, for instance, spatial mobility of agents is executed at a finer temporal scale as compared to that of local elections and the change in land use. Slum definition relies purely on occupancy density and does not account for other factors such as neighbourhood, housing structure, etc. The informality modelled is very general and it does not account for various professions and 


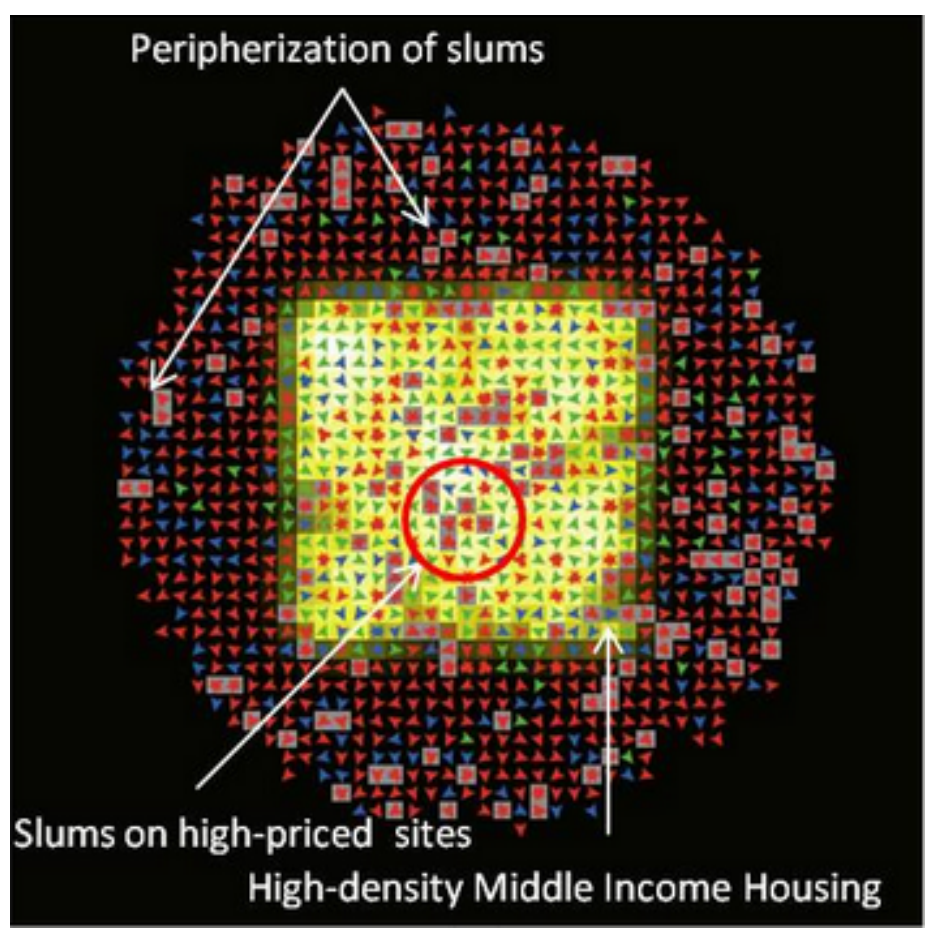

Figure 3.6: Slumulation Output. Source: Patel 2012.

skill sets. Slumulation also does not account for religion and ethnicity in slum policies. The Human Development Index (HDI) is not factored into slum formation leaving out the impact of education and longevity. The model assumes that economy expanding at its "potential" will continue growing at the same pace unless it is disturbed. This concept does not explain the period of "boom" and "bust" experienced in the economy. Slumulation does not consider the impact of existing slums as an attractive force for low income migrant population. Slumulation excludes the impact of "income-effect" and "substitution-effect" (due to demand shift-up) on house pricing. Slumulation does not capture complex interactions such as developers competing amongst themselves, which impacts the land prices. Further, the model does not implement the vote bank politics which will impact all "What-If" scenarios related to politics.

All the above models, including Slumulation implement an iterative search process to match the agents with an appropriate housing. However, it is a given in Social Science that such a search process does not exist and each slums has certain "filters" based on religion, ethnicity, profession, etc. A household or individual matching those "filters" directly settles in the respective slums. An iterative search process is not only computationally expensive, but it does not represent a realistic agent behaviour 


\subsection{Discussion}

In this chapter we have reviewed the state-of-art in modelling of slum dynamics, identified key factors for the emergence and growth of slums, and presented a set of standardized evaluation criteria which can be applied to urban and slum growth models. This chapter also presents a taxonomy of key factors that influence the growth and emergence of slums (table 3.1), which turned out to be useful to characterise data, identify proxies for missing data, steer directed data collection, and model building. Finally, we have reviewed existing simulation models using the standardized evaluation criteria and identified key open research questions.

Specifically, with respect to the model evaluation, we have considered three important features: level of detail, growth factors, robustness and implementation of the model, analysed in section 3.1 and 3.2. Conceptually, the modelling of slum formation in a spatial environment is important as the growth of slums is influenced by the spatial pattern. Based on our findings from the literature review we have identified that most models (discussed in section 3.3) lack an explicit spatial component or are coarse-grained. Furthermore, while most of the models have implemented population dynamics, they do not implement household structure and composition in determining location choice of slum dwellers. In the context of developing countries, the role of social groups and principle of homophily is also crucial and needs to be incorporated in any slum growth model. With respect to slum formation, all reviewed models took different approaches to study the emergent phenomenon of slum formation, however most of them lack a clear definition of slums. Some of the models lack key growth factors such as local politics, infrastructure and the role of informal economy. Slumulation is the only model which attempts to capture the role of local politics. However, further work is required to implement the vote bank politics in its entirety. All the models reviewed in this chapter use an iterative search process to match agents and housing, which may not represent the reality as it ignores the role of social capital in the settlement of the urban-poor. The models presented in this chapter include one or some of the above features but none of them integrates all these important features into a comprehensive model.

Based on the insights gained from the analysis of existing slum models and other slum studies, we finally identify a number of key future research topics in slum dynamics. To begin with, it is important to study and assess the resilience of slums as they exhibit vastly different levels of resilience. While many slums are vulnerable to forced evictions, temporary jobs, and constant migration; few slums like Dharavi (Mumbai) and Kibera (Nairobi) are able to respond 


\section{REVIEW OF SLUM MODELS}

and recover from external shocks and stress. Understanding what drives this difference in resilience is crucial for policy makers and urban planners. Furthermore, each slum in a city serves a specific function and they provide low cost housing for individuals immigrating from rural areas. A slum can be considered successful if it can assist a large number of urban-poor to increase their socio-economic status and well being. However, further work is required before we attempt to quantify the success of each slum and its contribution to the society, an area of work well suited to ABMS approach due to its ability to model the effect of individual and individual to environment interactions on a systemic level. We can model the influence of unquantified second order and third order contributions by the slum dwellers such as the availability of cheap labour that allows greater earning potential for the employers, and the reduced costs due to the lower quality of services being delivered to the slum dwellers. An ABMS approach can also be used to study the response of slums to internal and external forces such as lack of infrastructure, forced evictions, temporary jobs, and constant migration by incorporating all the growth factors discussed in this chapter. In addition, it is also essential to incorporate slum typology in the analysis as each slum in a city have distinct characteristics and hence cannot be perceived under one rubric. In order to answer the above key research question, we need to understand the key differences in dynamics between slum and non-slum households. Further, to understand resilience, we need to model the spatial movement of slum dwellers based on their social, economic and environmental satisfaction. Therefore, in Dynaslum we adopt a bottom-up approach (from an individual slum dweller to the emerging slum patterns) and focus on modelling the population dynamics and inter-slum migration of slum dwellers. The increasing adoption of GIS in urban planning and development has strengthened the case for highly granular spatial models for simulation of slum dynamics [87, 68, 88].

Finally, the last decade has seen a deluge of data of urban systems and slums are no exception. Researchers can now use different data sources such as census data, proxy data, independent surveys, and accurate remote-sensed data for land-cover changes. In order to model or understand the growth and emergence of slums there is a need for mechanisms which are capable of extracting key patterns at different hierarchical levels and scales from these huge data sets. Pattern-oriented modelling can then be used to optimize the model complexity and to reduce uncertainty in model structure and parameter [83]. The above approaches to study slums have not been modelled yet and using such a modelling approach is considered to be a useful support for effective policy intervention in slums. 


\section{Chapter 4}

\section{Slums in Bangalore}

In chapter 2 we have described the different definitions of slums (with a special focus on India) and how it guides the development of Dynaslum. We have also highlighted the impact of having multiple definitions of slums in a single city. Further, in section 2.2 we have provided a general characterization of slums across the developing countries based on which we have identified a set of growth factors for the emergence of slums (section 2.3). We find that it is crucial to model household composition and structure as they influence the residential choice of slum households. In chapter 3 we have reviewed the current state of art in slum growth models and find that current models lack a comprehensive population dynamics component. We also find that these models do not model the human behaviour accurately with respect to residential choices. Therefore, the key focus of Dynaslum is to bridge the gap in the current state of art and develop a robust household dynamic component coupled with a novel residential choice model. In this chapter we look into the present conditions and status of slum households living in Bangalore, India using a unique data collected from field survey of 37 slums. We also provide empirical evidence to show that household structure and socio-economic conditions play an important role in determining the choice of slum for the household. In section 4.1 we briefly describe the local governance and spatial structure of slums in the city of Bangalore and the current status of low cost housing. Further in section 4.2 we explain the novel dataset from the field survey of 37 slums and provide quantitative information about slums in Bangalore. The dataset will be used further to calibrate and validate Dynaslum. 


\subsection{Local Governance and Distribution of Slums in Ban- galore}

Bangalore is the capital of the state of Karnataka, and one of the fastest growing cities in India. Bangalore is the fifth largest city and third most populous city in India, located on the Deccan plateau in the south - east part of Karnataka [1]. It is a multi-cultural city permeating class, religion and language. The city of Bangalore has $21.5 \%$ of the total slum population in the state of Karnataka, and every fifth person within the city limits lives in a slum. The population living in urban slums in Bangalore has doubled in a decade and this poses a serious challenge to urban planners and policy makers [1]. According to the Karnataka Slum Development Board, the city has around 597 slums. However, the Association for Promoting Social Action estimates that the city has over 1500 slums which are not counted by the government, illustrating the importance of the issue. The case is representative for a society in a developing country characterized by multiple religions, caste and languages.

\subsubsection{Local governance structure of Bangalore}

According to the Karnataka Municipalities Act, 1964, the Directorate of Municipal Administration oversees the administration of 7 City Corporations, 44 municipal councils, 94 Town Municipal Councils, 68 Town panchayats. The District Urban Development Cells monitors these urban local bodies. Bruhat Bengaluru Municipal Palike (BBMP) is the municipal corporation responsible for all municipal activities of Bangalore. The area covered by it is around $790 \mathrm{~km}$ square in 198 wards. For the purpose of administration BBMP has been divided into 9 zones. Apart from BBMP there are 10 other statutory authorities with overlapping roles (see figure 4.1).

\subsubsection{Spatial Distribution of Slums in Bangalore}

In this section we measure the distribution of slums by calculating three spatial statistic measure - Spatial Imbalance, Standard Distance and Nearest Neighbour Index to quantitatively understand the patterns of slums in Bangalore. The spatial imbalance can be defined as the distance of the centroid (often known as "Mean Center") of the distribution of slums from the centroid of the city.

We also use another statistic known as the Standard Distance (SD) to measure the distribution of slums around their centroid. "Standard Distance $(\sigma)$ measures the degree to which 


\section{GOVERNANCE STRUCTURE}

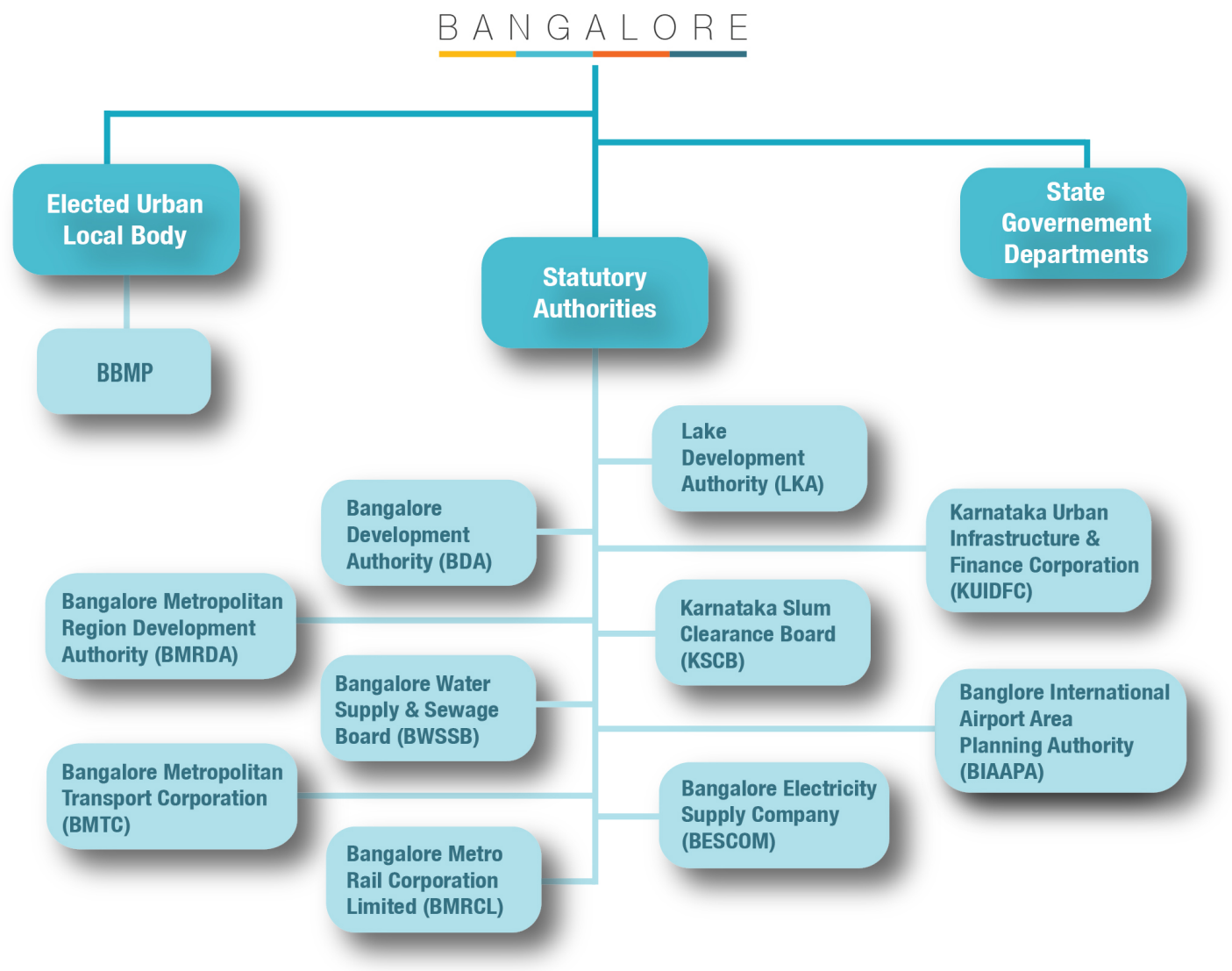

Figure 4.1: Governance structure in the city of Bangalore.

features are concentrated or dispersed around the geometric mean centre. It provides a single summary measure of feature distribution around their centre (similar to the way a standard deviation measures the distribution of data values around the statistical mean)".

In general, assuming a spatial normal distribution (SND) the spatial orientation of an input feature is focused towards the centroid with lesser features further from the centroid. The standard norm is that circle with radius $=1 \sigma$ includes approximately $68.27 \%$ of the features, radius $=2 \sigma$ contains $95.45 \%$ of the feature while a circle with radius $=3 \sigma$ will cover approximately $99.73 \%$ of the features in the cluster. We run this test on the slum data from Bangalore to test for the spatial normality in the distribution of slums. The value of $\sigma$ was $6.3 \mathrm{~km}$ in case of Bangalore, which served as a radius for the circle centred at the centroid of slums as demonstrated in the figure 4.3. Further, as shown in table 4.1, the normality test indicates that 


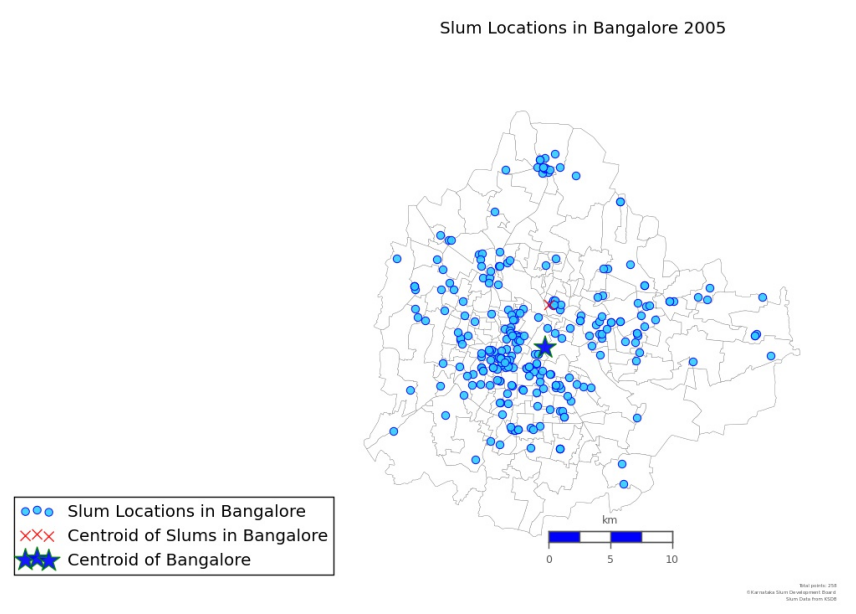

Figure 4.2: Slum Distribution across Bangalore in 2005 (Source: Karnataka Slum Development Board).

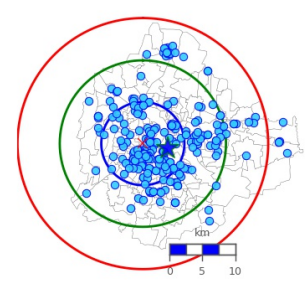

-00 Slum Locations in Bangalore

$x \times x$ Population weighted Centroid of Slums in Bangalore Centroid of Bangalore

Figure 4.3: Spatial Normal Distribution of Slums across Bangalore in 2005 (Source: Karnataka Slum Development Board).

most slums are clustered near the slum centroid while there are less number of slums in the periphery of the city.

Further, we conduct the Doornik Hansen test of multivariate normality on slum locations in Bangalore. The null and alternate hypothesis is formulated as: 


\begin{tabular}{|l|l|l|}
\hline Weighted SD & Estimated Slums & Observed Slums \\
\hline $6.3 \mathrm{~km}(1 \sigma)$ & $68.27 \%$ & $70.23 \%$ \\
$12.6 \mathrm{~km}(2 \sigma)$ & $95.45 \%$ & $94.54 \%$ \\
$18.9 \mathrm{~km}(3 \sigma)$ & $99.73 \%$ & $98.81 \%$ \\
\hline
\end{tabular}

Table 4.1: Comparison of expected and observed number of slums to prove SND slums in Bangalore.

- $H_{0}$ : Longitude and Latitude of slums follow spatial normal distribution.

- $H_{a}$ : Longitude and Latitude of slums do not follow spatial normal distribution.

The Doornik Hanson test statistic, $\chi^{2}(8)=19.323$, which shows that we cannot reject $H_{0}$ for spatial normal distribution at $90 \%$ confidence $(p \geq 0.1)$. The Mardia's statistic (test for kurtosis and skewness ), $\chi^{2}(1)=1.769$ and $\chi^{2}(20)=23.528$, indicates that we cannot reject $H_{0}$ for spatial normal distribution at $90 \%$ confidence $(p \geq 0.1)$.

The aim of testing for multivariate normality of slum distribution is to improve the understanding of growth and emergence patterns of slums and provide basis for quantitative validation of Dynaslum. The results presented here are very similar to the city of Ahmedabad, presented in Slumulation [6]. As data becomes available for other cities, it would be interesting to find similarity or differences in growth patterns of slums across developing countries, which could then provide clues about latent processes that drives the slum sprawl in cities.

\subsubsection{Spatial clustering of Slums in Bangalore}

In this section we calculate the Nearest Neighbour Index (NNI) to explore the underlying spatial process that may lead to a random, clustered or dispersed pattern of slum formation in Bangalore.

NNI is "expressed as the ratio of the Observed Mean Distance to the Expected Mean Distance". We use the $z$ score and significance testing in Q-GIS to evaluate if the observed dispersion or clustering is random or dictated by an underlying spatial process. The null hypothesis for the nearest neighbour analysis is that the points are randomly distributed (CSR). The NNI for the slums in Bangalore was 0.4584 identifying the presence of spatial clustering. As shown in figure 4.4, the Observed Mean Distance is 0.0069 and Expected Mean Distance is 0.015 . The results indicate that the location of slums are not random as the mean distance between nearest neighbours is small. As indicated in figure 4.4, the value of $z$ score $(-16.6)$ and 


\section{SLUMS IN BANGALORE}

$p$ value less than 0.01 indicates that there is less than a $1 \%$ likelihood that the observed spatial clustering is random.

Therefore, the hypothesis that the spatial patterns are random can be rejected, which in turn means that the spatial formation of slums in Bangalore may be due to some latent underlying factors (such as segregation, social network). This finding could be interesting for urban planners and policy makers as they can now target these clusters to spread education or skilled labour programs. Similarly, these clusters may also guide the development of efficient (high utilization) of services like water supply, transportation and other basic services. Further, we use Kernel Density Estimation (KDE) method to identify slum clusters (often referred as "hot spots") in Bangalore. Figure 4.5 shows the regions in Bangalore where slums are grouped or clustered. Most "hot spots" are located in the centre of the city while there are few "hot spots" in the Northern and Southern parts of Bangalore.

Comparing the "hot spots" with known land-use of Bangalore, it is evident that these clusters are in the proximity of important job centres and land parcels which are not suitable for development (e.g. construction places, factories, railway tracks) which may have influenced the growth and emergence of slums in these locations. Nevertheless, to derive a correlation between the slum clusters and spatial features (such as texture, entropy), land use data must be gathered in future by processing high resolution satellite images to identify slums. It could also advance the calibration and validation of the model with the addition of these empirically derived parameters.

\subsubsection{Status of low-cost housing in Bangalore}

In 2011, Bangalore Development Authority (BDA) issued a notification, to construct 13,172 flats in 13 places on the outskirts of the city. The price were fixed at INR 0.75 million for a two room flat which was later to revised to INR 1.7 million, making it much more unaffordable. The housing program failed due to high cost and also because the flats were located in the periphery of the city which did not factor the livelihood option of the urban poor.

\subsection{Status of Slums in Bangalore using the Survey Data}

The survey employed in this analysis was conducted in the year 2010, by Fields of View (FoV), a non-profit organisation based in Bangalore, India. The aim of the survey was to investigate the issues of living wages for the urban poor, the household life cycle in dense urban areas and 


\section{Average Nearest Neighbor Summary}

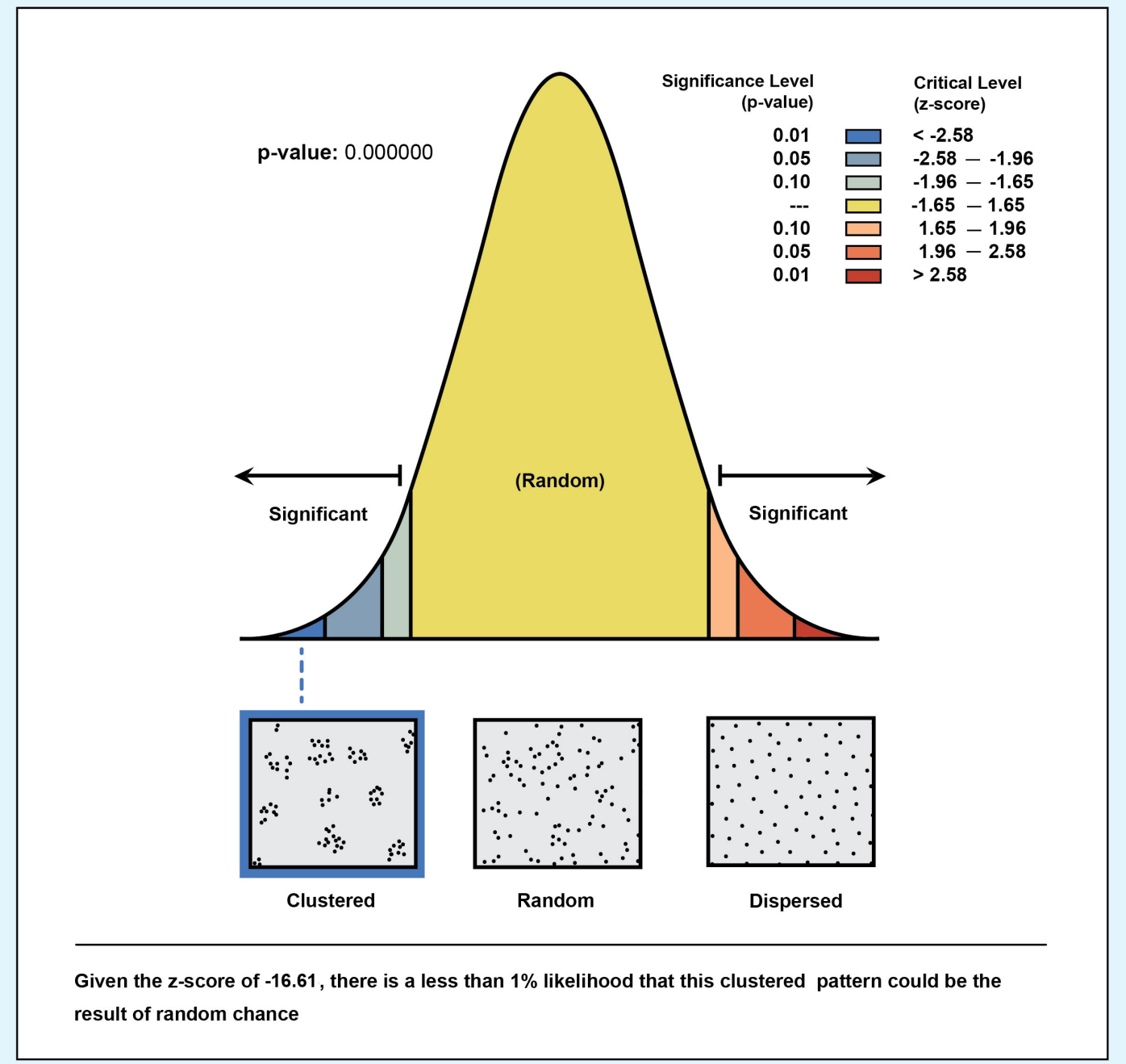

\section{Average Nearest Neighbor Summary}

\begin{tabular}{|r|l|}
\hline Observed Mean Distance & 0.00690646570765 \\
\hline Expected Mean Distance & 0.0150664217898 \\
\hline Nearest Neighbor Index & 0.458401192001 \\
\hline z-score & -16.6102108101 \\
\hline Number of points & 257 \\
\hline
\end{tabular}

Figure 4.4: Spatial Clustering of Slums in Bangalore in the year 2005. 


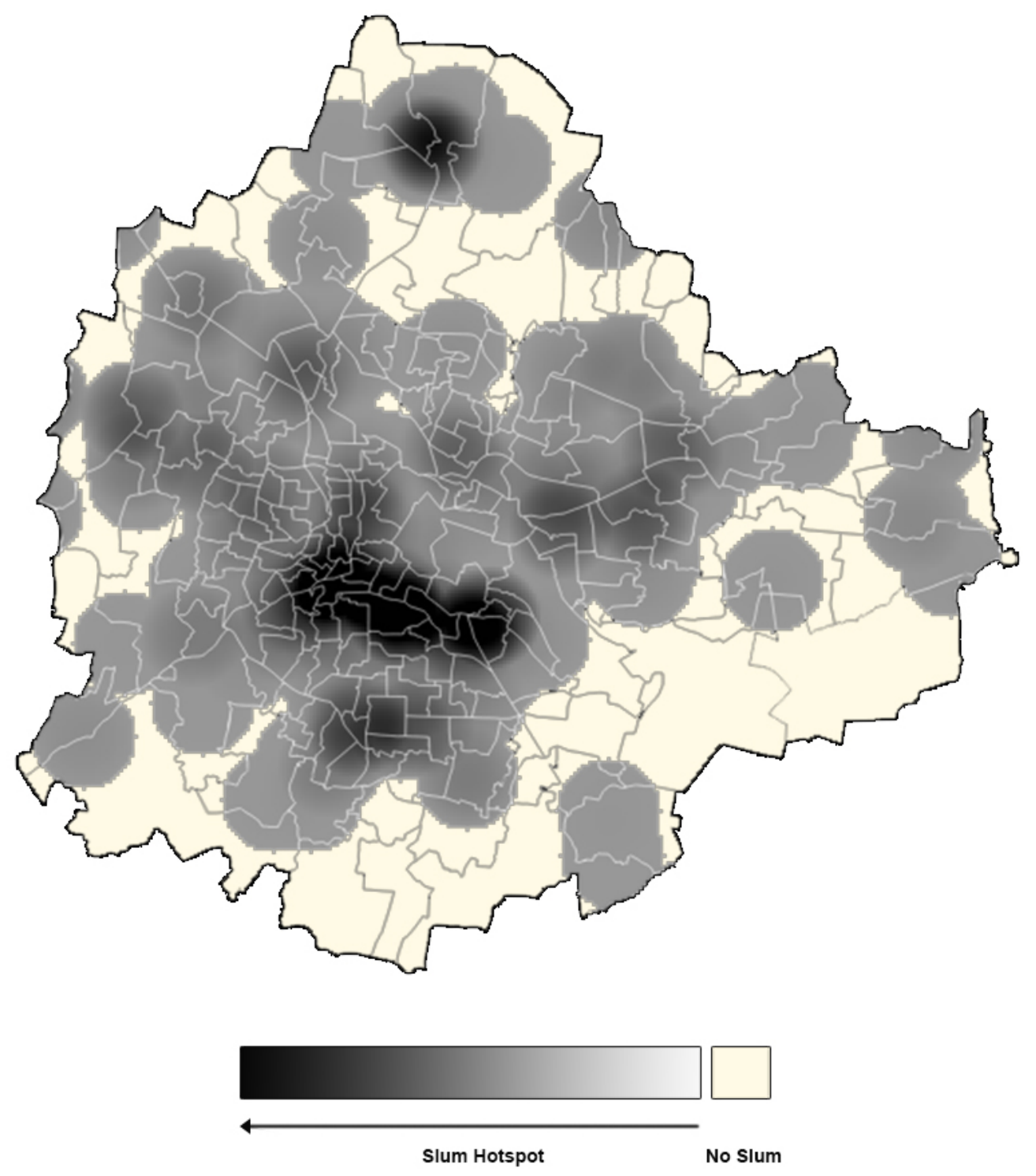

Figure 4.5: Hot Spots of Slums in Bangalore in the year 2005.

the relationship between well-being and income for the urban poor. The survey used a random stratified sample of 37 slums with a target of 1100 households, with the following stratification criteria:

- Age of the Slum (Old, New), 
- Location in the city (Core, Periphery, North, South, East, West);

- Land Type (Public land, Private land);

- Declaration Status (Declared (notified), Not Declared (non-notified)) ${ }^{1}$;

- Major Linguistic Group;

- Major Religious Group;

In each slum, the survey covered $10 \%$ of the households, choosing every tenth household, based on the layout patterns of the slum constructed by the slum dwellers themselves, as no formal layout plan is available. The survey team was composed of women living in the slums that were the focus of the study. The reasons for training slum residents to carry out the survey were two-fold. First, using local slum dwellers as surveyors, created a level of trust between people who are being surveyed. The degree of comfort being able to confide to an insider rather than an outsider can provide accurate data that are not always captured in non-participatory methods. Second, it ensures that the survey team understands the context of the survey and the issues involved, so that they could be more nuanced in their approach, as opposed to an outsider. Given women are often considered to be less threatening and more trustworthy and reliable, the surveyors were all women, who conducted the surveys in teams of two. Each survey comprised of 242 questions from 1114 households, generating around 300,000 data points. The survey data was entered into a database and checked for possible reporting errors. The average family size of slum households in the survey was reported as 4.9. The Mean Gross Monthly Income in the survey is 3472 INR with $60 \%$ of the respondents earning below the average. Majority of survey respondents (67\%) are Hindus. About $20 \%$ of the respondents are Muslims and $8 \%$ are Christians. The native language of $45 \%$ of sample respondents is Tamil, while 17\% speak Kannada and 15\% speak Telugu Caste has been a dominant factor in residential segregation in most Indian cities, however, in the case of slums in Bangalore a lack of accurate data hinders any further analysis. Around $46 \%$ of the respondents in the survey did not disclose their caste and it is expected that majority of them belong to the lower caste. Therefore, since undisclosed caste data in this case can be treated as missing not at random, we exclude caste from any further analysis. In the following sections we describe the household characteristics of slum dwellers in Bangalore based on the survey data.

\footnotetext{
${ }^{1}$ In India, "non-notified" slums are not officially recognized by city governments; they suffer from insecure tenure and poorer access to basic services than "notified" (government-recognized) slums. (See The Karnataka Slum Areas Act 1973.)
} 


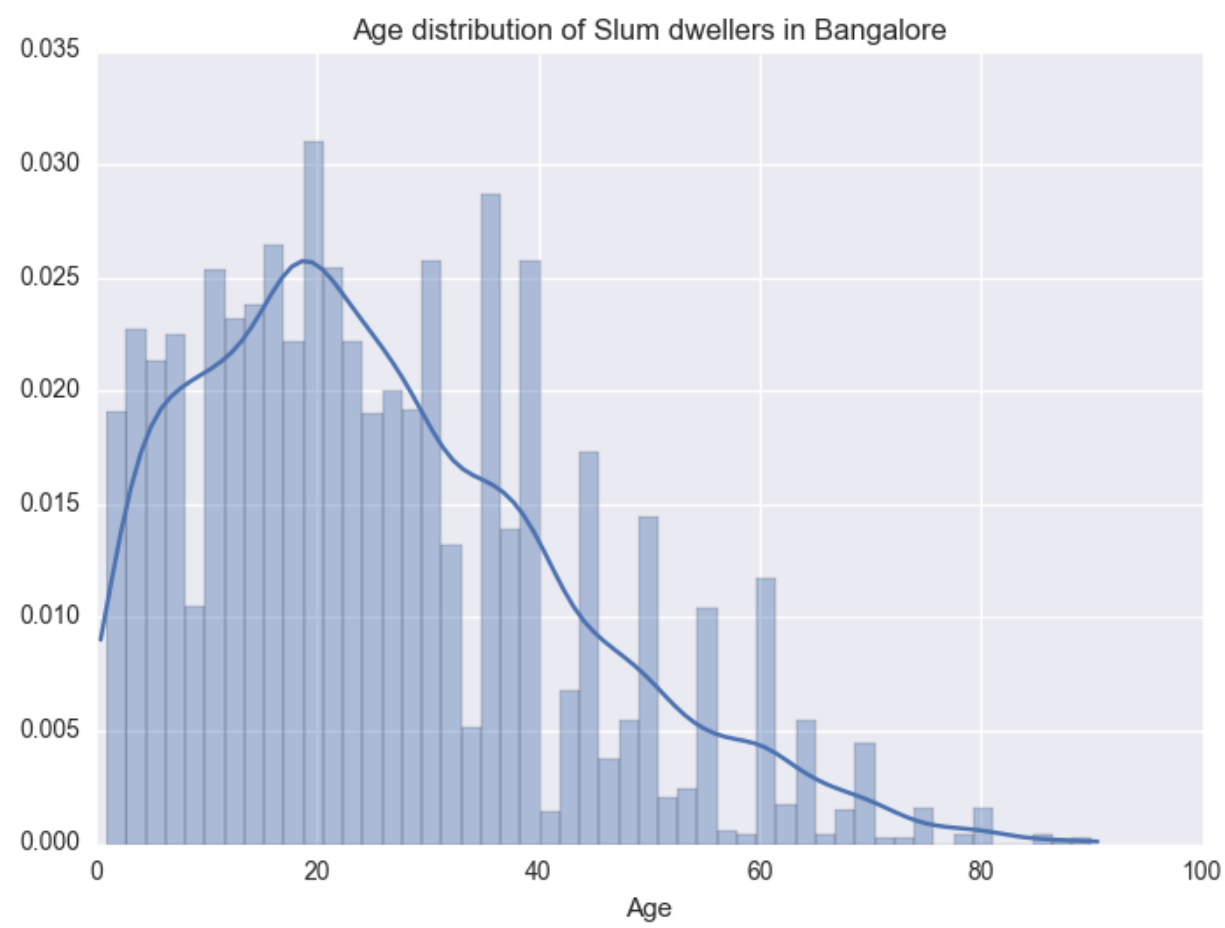

Figure 4.6: Age Distribution across the 37 slums (Refer table A.2 for list of slums) in Bangalore.

\subsubsection{Demographic Profile of Households}

\begin{tabular}{|l|l|l|}
\hline Demographic Variable & Classification & Proportion of Respondents \\
\hline Gender & Male & 49.01 \\
& Female & 49.35 \\
& Transgender & 0.19 \\
\hline Age & Under 18 & 34.93 \\
& $18-24$ & 17.64 \\
& $25-34$ & 17.89 \\
& $34-60$ & 25.34 \\
& 60 above & 4.19 \\
\hline
\end{tabular}

Table 4.2: Gender and Age Distribution across the 37 slums (Refer table A.2 for list of slums) in Bangalore.

The mean and median household size in the slums of Bangalore is around 5. The findings conclude that $25 \%$ of the families have a household size of up to 4 members and $75 \%$ of the slum 


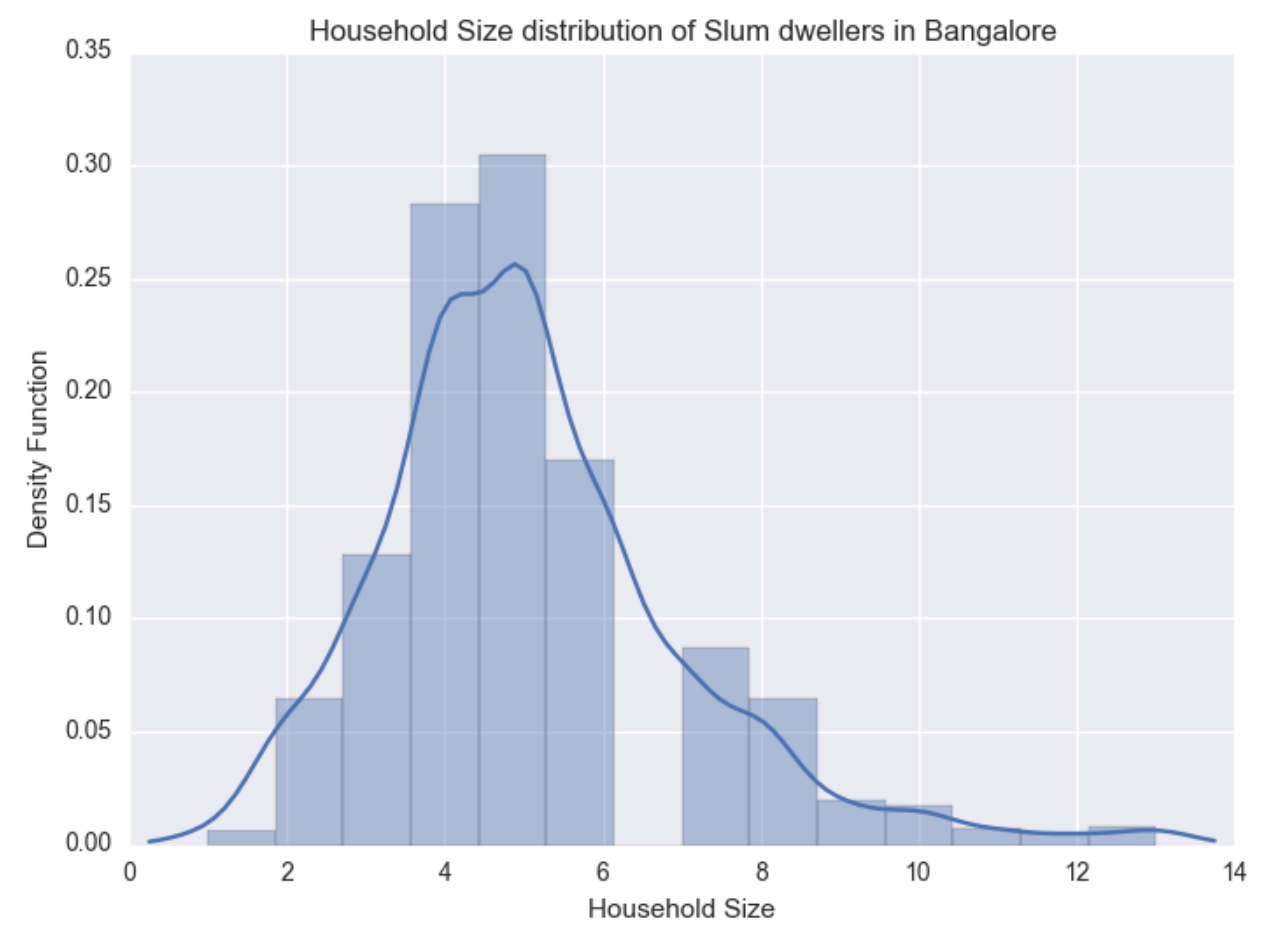

Figure 4.7: Household Size Distribution across the 37 slums (Refer table A.2 for list of slums) in Bangalore.

dwellers have a household size of up to 6 members. The maximum household size in the sample population is 13. Figure 4.7 shows the household size distribution across the 37 surveyed slums in Bangalore. Table 4.2 indicates that the gender ratio(female to male ratio) is around 1, which is different to the trend in non-slum urban households where there are around 966 female per 1000 male. Table 4.2 also shows that the population in slum is young with around $35 \%$ of the sample respondents under the age of 18 and around $70 \%$ of the sample respondents under the age of 35. The age distribution is consistent with the data from Census of India 2011 [14].

\subsubsection{Age of Marriage}

In the slums of Bangalore, the average marriage age for men is 24 and 17 for women. This is lower than the average of non-slum urban households in Bangalore, where the average age of marriage is 27.5 for men and 24 for women [14]. The median age of marriage has been rising in India. However, $49 \%$ of all women in the sample population are married before the age of 17 . The median age at first pregnancy in slums of Bangalore is around 18 years which is significantly 


\section{SLUMS IN BANGALORE}

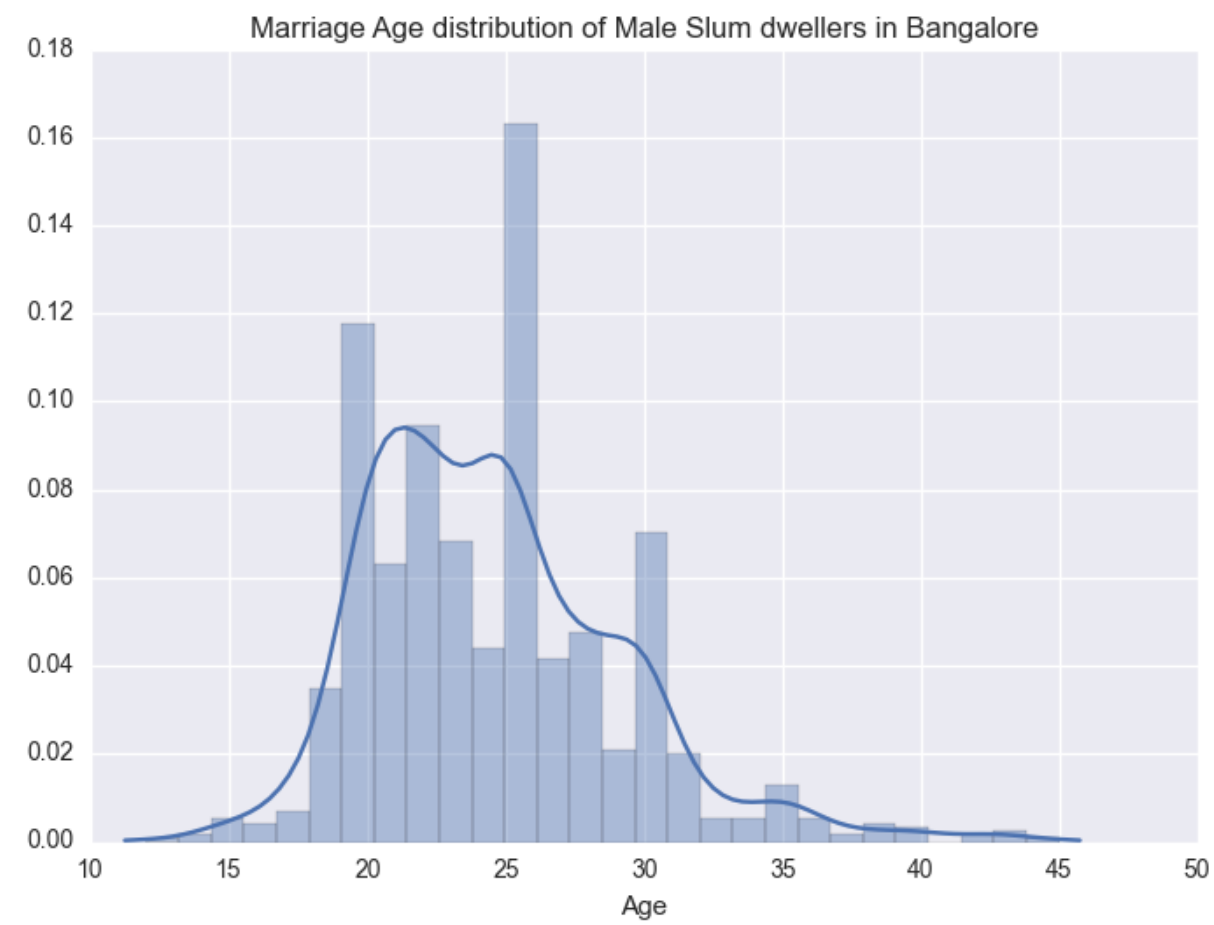

Figure 4.8: Age Distribution of Marriage for men in slums of Bangalore.

lower than the median age of 25 years for non-slum urban households in Bangalore [14]. Figure 4.8 and 4.9 shows the age distribution of first marriage for men and women respectively. The survey also indicates low divorce rates (around 1.3\%) in the slums of Bangalore. However, this could be also due to under reporting of divorce.

Figure 4.8 and 4.9 demonstrates a view of the household life cycle (HLC) from the perspective of an individual, showing the age distribution at which couple formation occurs. In Dynaslum, a new household is formed from an existing household when (discussed in chapter 6) either (a) leaving parental household to form a new single-person household; or (b) couple formation leading to independent living.

\subsubsection{Literacy and Occupation}

Age categorisation was done based on the constraint that literacy and occupation data need to be analysed according to age. The first categorisation of age data was: $[0,6),[6,10),[10,14)$, $[14,18),[18,25),[25,30),[30,40),[40,60),[60,80),[80,95)$. The age group $[0,6)$ was added to identify the outliers in literacy and occupation data. 


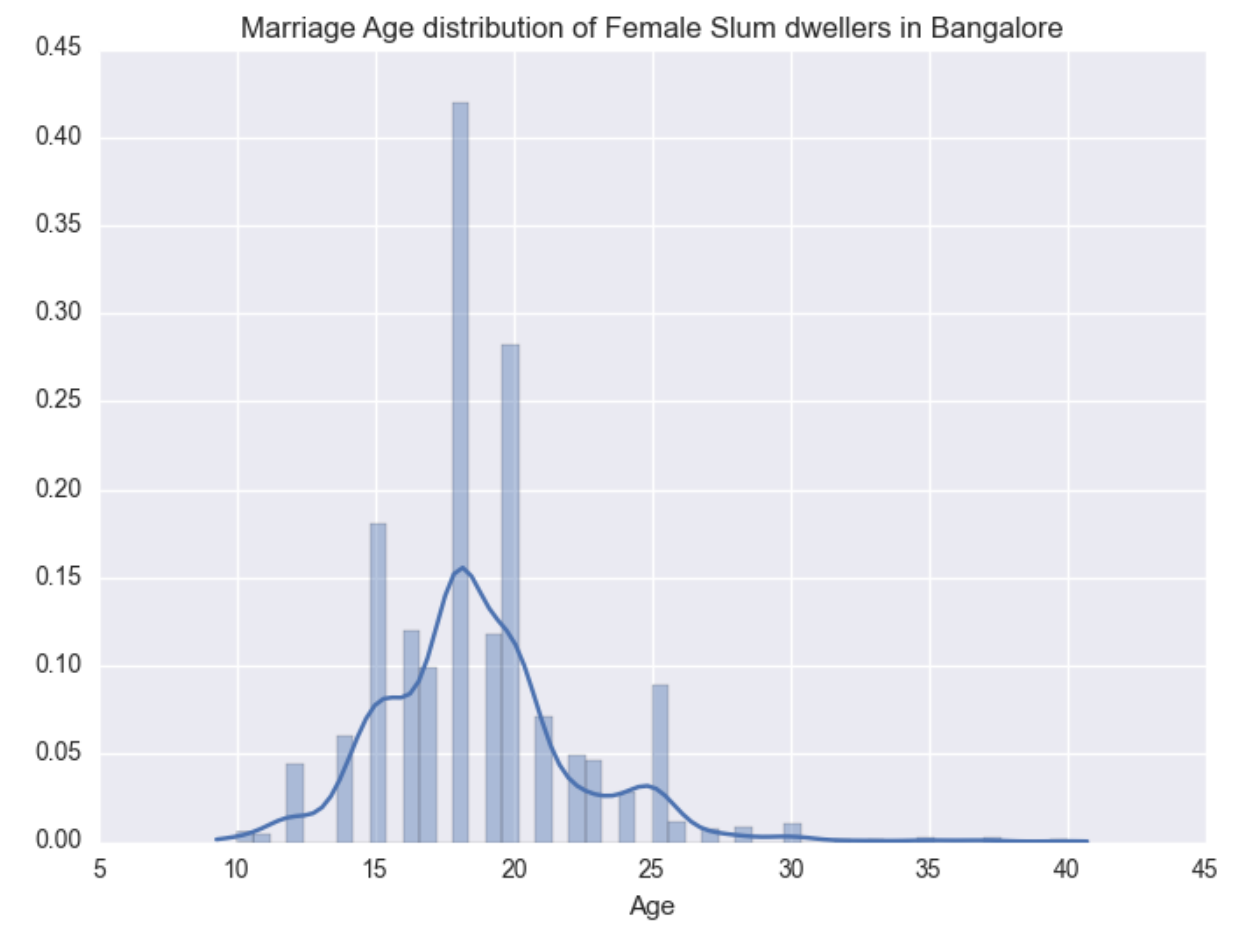

Figure 4.9: Age Distribution of Marriage for women in slums of Bangalore.

Age groups from 6 to 18 were further divided into three bins: $[6,10),[10,14),[14,18)$ to identify incidence of child labour. For the second version of the categorisation, the age group $[18,25)$ and $[25,30)$ were merged to form $[18,30)$. The age group following this are in increments of 10. Furthermore, it was identified that splitting the age groups from 6 to 14 into two groups [6-10) and [10-14) would not be useful when analysing occupation data, but very important to analyse literacy data. Therefore, the sample population was categorised into two age groups, one for literacy data analysis and one for occupation data analysis.

1. Age Bins for Literacy Data Analysis: $[0,6),[6,10),[10,14),[14,18),[18,30),[30,40),[40,50)$, $[50,60),[60,70)$, Greater than 70 .

2. Age Bins for Occupation Data Analysis: $[0,6),[6,14),[14,18),[18,30),[30,40),[40,50)$, $[50,60),[60,70)$, Greater than 70 .

Figure 4.10 shows that the youth in the slums of Bangalore lack higher education or technical training required for employment in the formal sector. Most of the sample respondents in the 


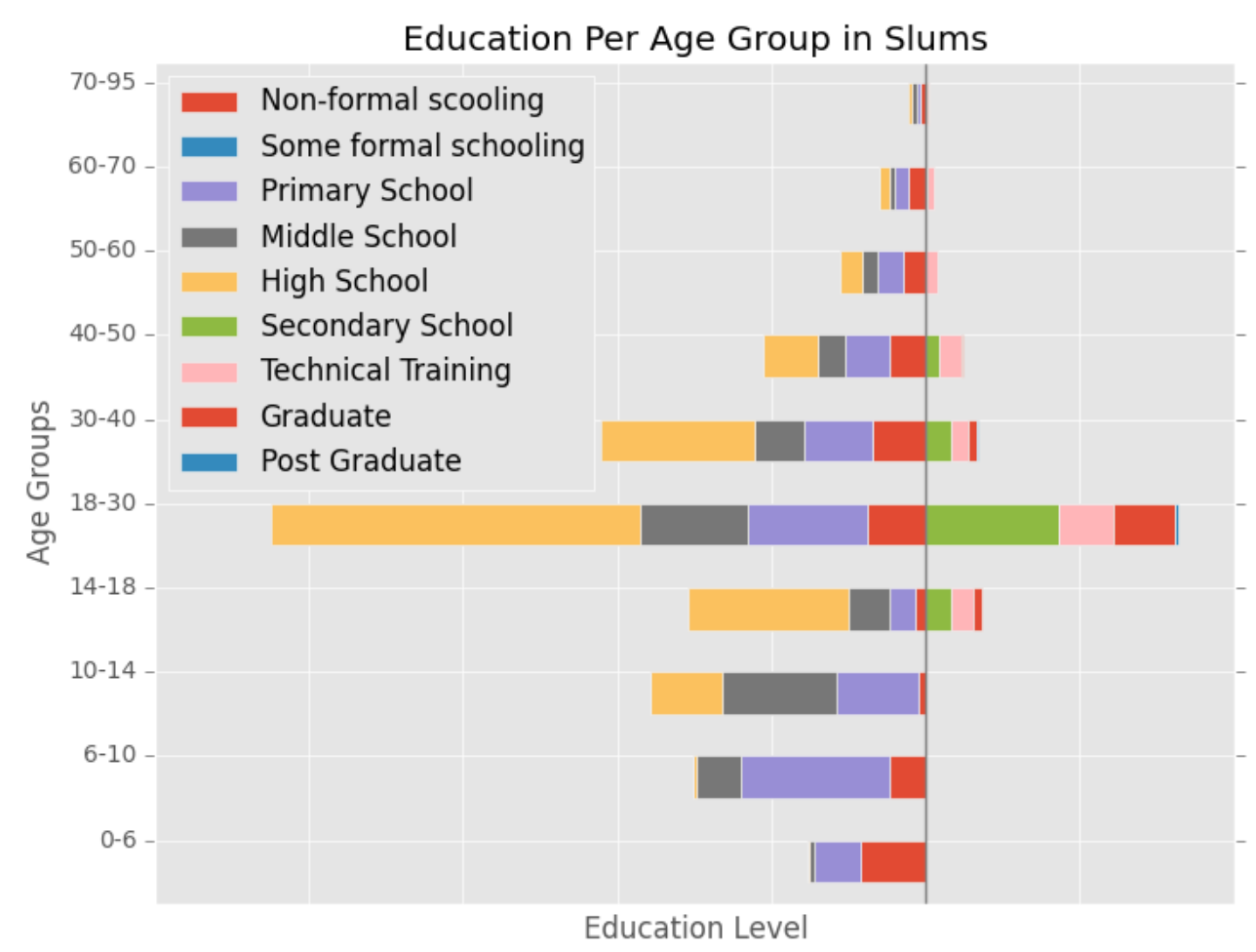

Figure 4.10: Education Levels across age groups in the 37 slums of Bangalore.

age group $[14,30)$ are high school drop outs. Around $7 \%$ of the respondents in the age group $[18,30)$ have finished secondary school or higher, thus severely restricting their employability in the formal job market. This reflects in figure 4.11 which shows the number of respondents employed in each occupation category based on the age group defined above.

The finding indicates that most slum dwellers are employed in the informal sector, primarily working as domestic help or as manual labour in the rapidly growing construction sector of Bangalore. Only $13 \%$ of the sample respondents are employed in the formal sector (White colour, blue colour and sales occupations). Figure 4.11 also indicates a low incidence of child labour in the slums of Bangalore (around $6 \%$ in the age group $[6,18)$ ).

\subsubsection{Income Distribution}

The income distribution (see figure 4.12 ) shows that $25 \%$ of sample respondents earn a monthly income of less than 2000 INR, out of which they spend $93 \%$ on basic amenities. Around $75 \%$ 


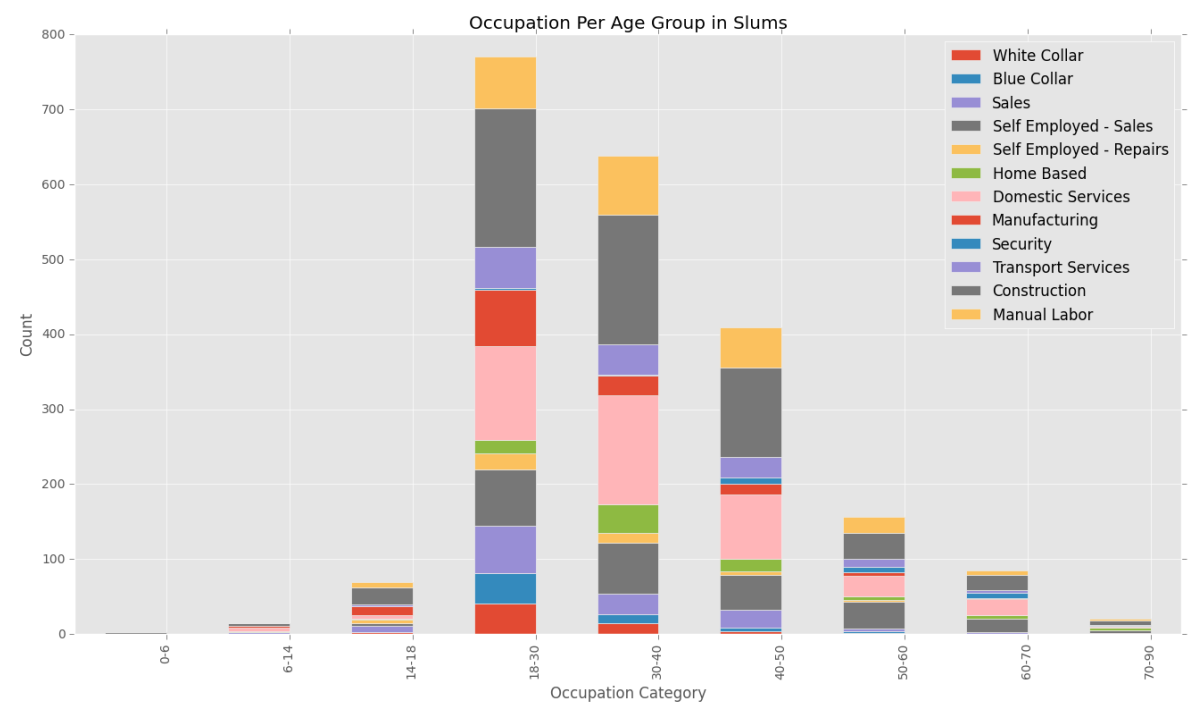

Figure 4.11: Occupation Categories across age groups in the 37 slums of Bangalore.

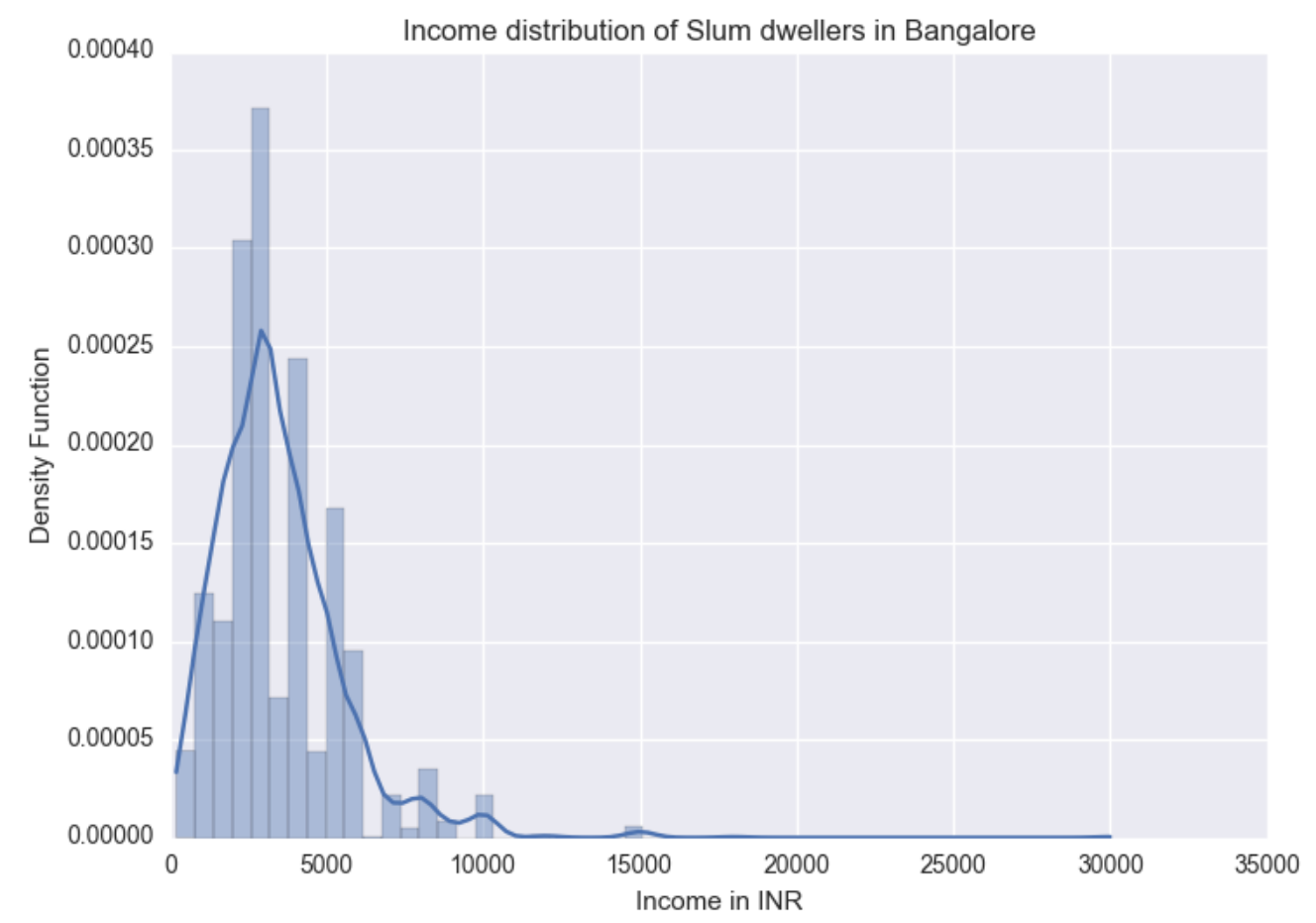

Figure 4.12: Income Distribution of Slum Dwellers in the 37 slums of Bangalore. 


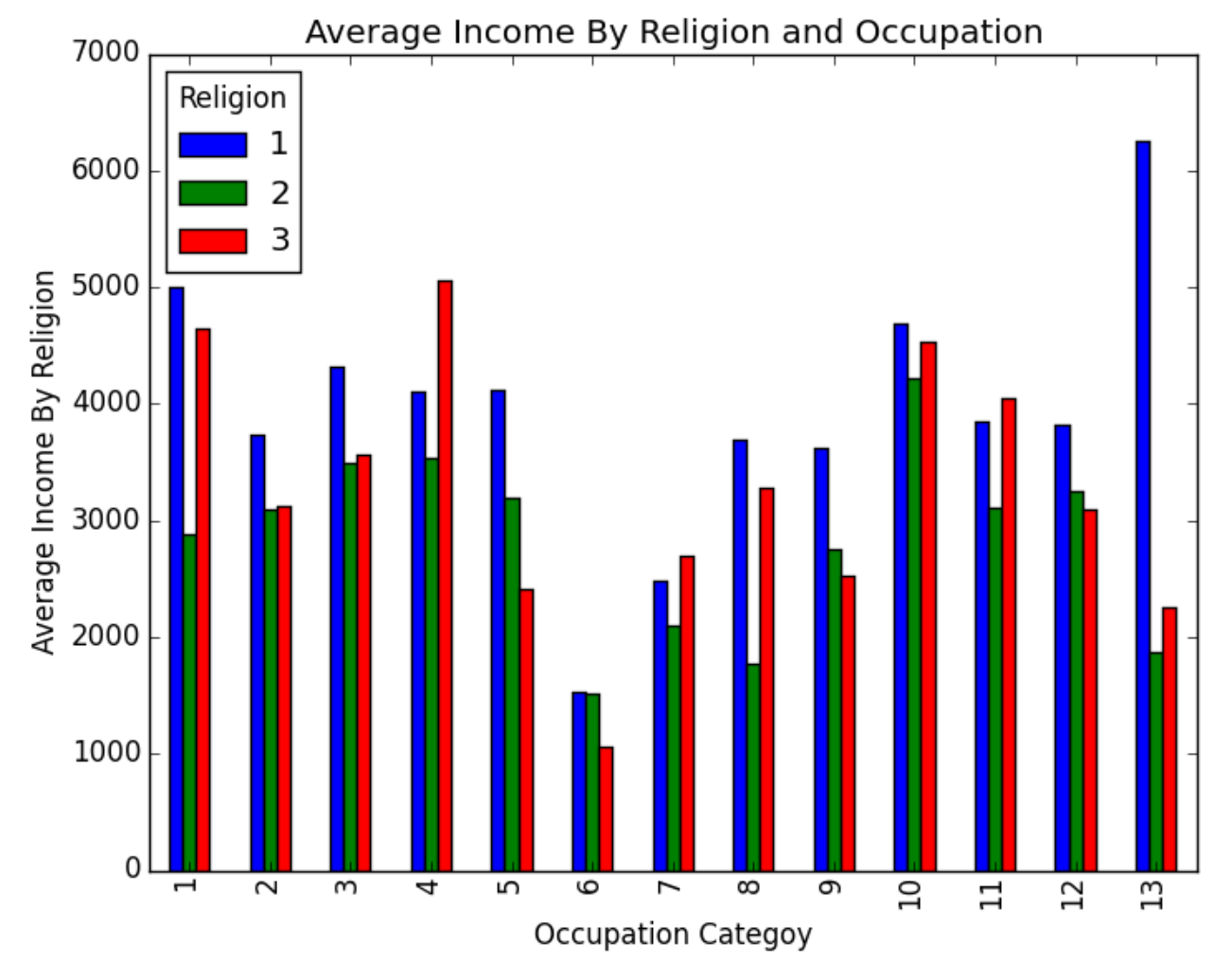

Figure 4.13: Income based on religion (1-Hinduism, 2- Muslim, 3-Christian) and Occupation(1 - Professional White Collar, 2- Professional Blue Collar, 3- Small Enterprises, 4 Self Employed - Sales, 5 - Self Employed - Repair, 6 - Home Based, 7 - Domestic Services, 8 Small Industry, 9 - Security, 10- Transport Services, 11 - Construction, 12 - manual labour, 13 Others)

of the sample respondents earn a monthly income lower than INR 4000 and spend $91 \%$ of their income. Around $13 \%$ of households earn more than INR 10,000 per month and spends around $77 \%$ of their income. The monthly median income of slum dwellers in Bangalore is around INR 3000.

Although most slum households are characterized by low income, there is a substantial amount of inequality in wages based on religion and occupation as show in the figure 4.13. In chapter 4 we analyse segregation and income inequality within the slums of Bangalore in further detail. The figure 4.15 indicates the ownership of assets among slum dwellers. 


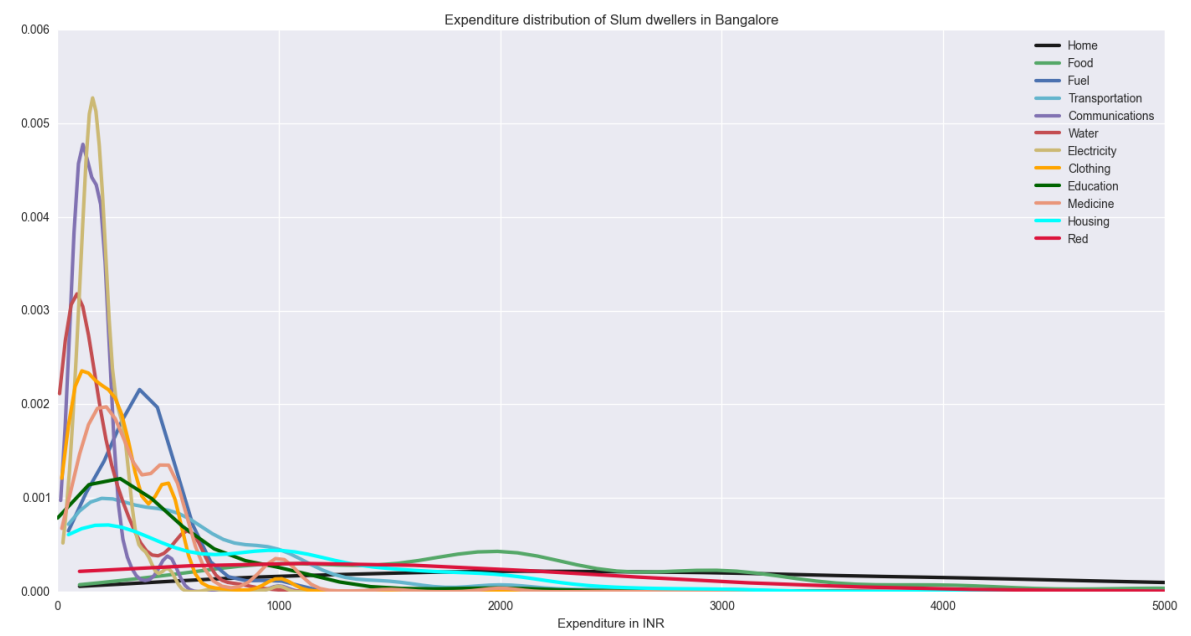

Figure 4.14: Expenditure distribution of slum households on various categories.

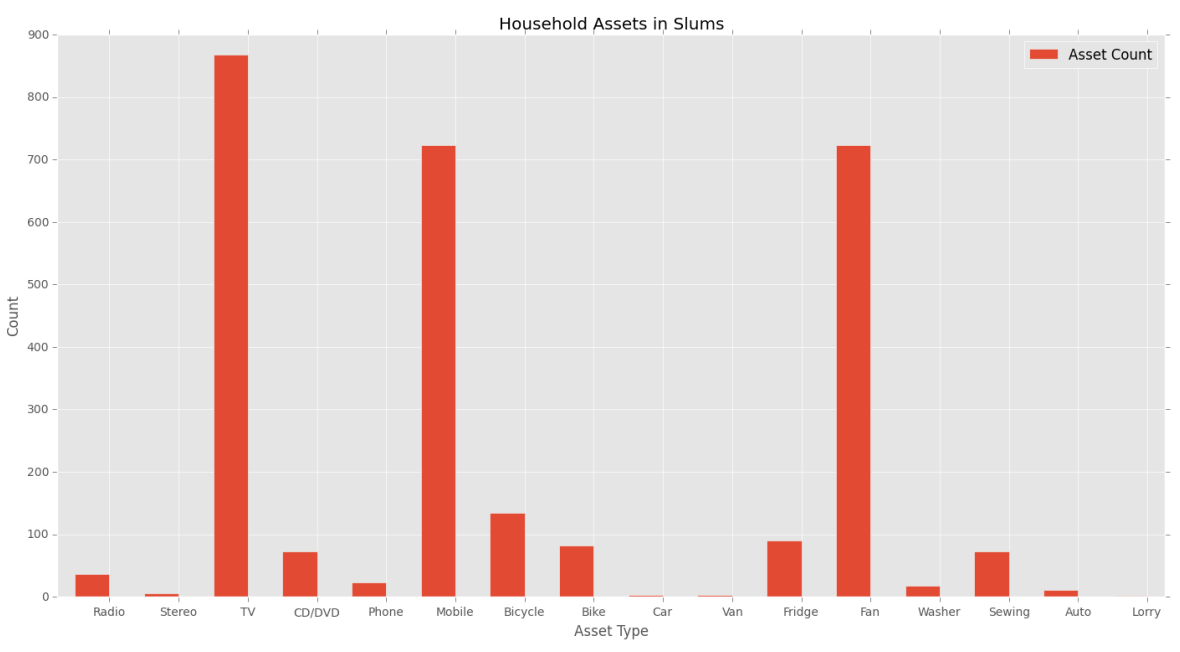

Figure 4.15: Asset ownership of slum households.

\subsubsection{Expenditure distribution}

Figure 4.14 and table 4.3 shows that the slum households have their majority expenditure on food items. The reasons for high percentage are low income level coupled with high food inflation based on the Consumer Price Index (CPI) which was 12.56\% during 2013. Hence, money available for other activities is very low. Education is high priority for some segment of urban poor, but high expenditure on education comes only from the top $10 \%$ in the slums. 


\section{SLUMS IN BANGALORE}

\begin{tabular}{|l|l|l|}
\hline Groups & Bangalore Urban & Bangalore Slums \\
\hline Food Items & 38.26 & 55.91 \\
Fuel & 8.82 & 3.12 \\
Housing & 26.04 & 7.23 \\
Clothing & 2.59 & 0.59 \\
Education & 2.37 & 1.11 \\
Health Care & 3.45 & 7.21 \\
Entertainment & 2.10 & 2.34 \\
Transportation & 9.56 & 11.21 \\
\hline
\end{tabular}

Table 4.3: Comparison of CPI between slums and non-slum households. Source:Central Statistics Office, February 11, 2014

\begin{tabular}{|l|l|}
\hline Housing Type & Percentage \\
\hline Kutcha & 40.50 \\
Semi-pucca & 19.74 \\
Pucca & 39.75 \\
\hline
\end{tabular}

Table 4.4: Housing types based on construction material.

The other key components which add to the expenditure are home appliances, rent, healthcare, and clothing. The expenditure data provides two key insights. First, the slum households in Bangalore spend more than the national average on transportation (conveyance). Second, the slum households pay almost twice the house rent than the national average. Therefore, we can conclude that basic facilities like food consumption, housing rental, health care and transportation consume bulk of their income leaving very little for other items.

\subsubsection{Status of Welfare Benefits}

In India, ration card is primarily used for purchasing subsidized food and fuel (cooking gas, oil, grains) and is a key document for the urban poor, providing proof of identity and access to public distribution system (PDS). The ration card is issued by the State Government limited to one per family. It is divided into "three categories: extreme poverty level (Antyodaya), below poverty line (BPL) and above poverty line (APL)" [14]. Analysis of ration card data from the slums in Bangalore shows that around $3 \%$ households have Antyodaya cards, $60 \%$ possess BPL cards and around $17 \%$ have the APL card. This indicates that vast majority of slum dwellers in Bangalore are below poverty line, placing a huge stress on the PDS in Bangalore. 


\subsubsection{Housing Status and Security of Tenure}

Housing is a prime economic resource and home ownership is essential for every household. In Bangalore, around $40 \%$ of the slum households have "Patta", which is an key certification given by authorities indicating title to the dwelling. This indicates majority of slum dwellers possess legal titles. Households with legal title to their dwelling usually live in Pukka houses. Around $20 \%$ of the households have Possession Certificate document and live in Semi Pukka houses. Rest $40 \%$ who have either migrated from other districts or other states do not have any proper ownership to land and live in Kutcha form of houses (See table 4.4).

\subsubsection{Rural-Urban Migration}

Bangalore has undergone rapid urbanization in past three decades and migration is becoming a serious challenge and an important component of population growth. Analysis of the migration data from slums show that $73 \%$ of migrants are from rural areas within Karnataka itself while the rest $27 \%$ migrated from the rest of India.

\subsubsection{Physical Infrastructure in Slums}

In the context of JNNURM and various other funded programs, Bangalore city has invested in infrastructure and slum upgradation. Government of Karnataka appointed BBMP as the key agency for this coordinated efforts in compliance with the implementation of 74th constitutional amendment which envisages urban decentralization and empowers local bodies. However, the survey data indicates that around $55 \%$ of the slum households depend on public taps for access to water. On average an individual household spends around 2 hours per day to fetch water for the household. In contrast, around $71 \%$ households have access to electricity through metered connection in comparison to $37 \%$ in rural Karnataka. Around 5\% households do not have toilets and $54 \%$ households share toilets with neighbours or use public toilets. Most slum households have their own kitchen (68\%) but around $50 \%$ use kerosene as fuel, $24 \%$ households use firewood and only $23 \%$ use LPG as cooking fuel. The construction material varies depending on the structure of the house. Most households have metal roofs built on walls with mud and flooring is usually brick based. 


\begin{tabular}{|l|l|}
\hline Reason to Change & Percentage \\
\hline Change in Job & 27.45 \\
Change in income & 15.49 \\
Friends or Relatives & 25.68 \\
Education of Children & 13.72 \\
Home based manufacturing possibilities & 11.76 \\
Access to mobility services & 5.88 \\
\hline
\end{tabular}

Table 4.5: Reason for wanting to change current slum settlement.

\begin{tabular}{|l|l|}
\hline Reason to Move & Percentage \\
\hline Close to work location(s) & 42.85 \\
\hline Friends or Family & 28.85 \\
\hline Education of Children & 14.28 \\
\hline Home based manufacturing & 5.71 \\
\hline Economic Reasons & 7.85 \\
\hline
\end{tabular}

Table 4.6: Reason for moving into current slum.

\begin{tabular}{|l|l|}
\hline Migration Reason & Percentage \\
\hline Marriage & 36.23 \\
\hline Employment or Opportunity & 57.89 \\
\hline Education & 5.63 \\
\hline
\end{tabular}

Table 4.7: Reason for migrating into slums of Bangalore.

\subsubsection{Choice of Slums}

In this section we provide an quantitative analysis of the reasons influencing the location choices of slum dwellers in Bangalore, based on the survey data. It can be observed from the table 4.7 that most (around 57\%) newly arrived migrants, first move into cheap temporary slums in search of employment from rural areas. After few years the slum households develop resilience and relocate to various slums in the city and settle in slums close to their work locations (around $43 \%$ ) or close to their friends or family (around 29\%). In Bangalore a large proportion of households (around 14\%) determine their choice of slums based on the education of their children. Further, in table 4.6 we see that there is another category of slum dwellers who would want to improve their house that reflects their change in job (around 27\%), earning (around $15 \%$ ) and status in the society (around 39\%). Therefore, based on their economic condition, we find that household structure and composition and the social network plays a crucial in 
the residential choice of slum dwellers. We will further discuss residential choice in detail as a separate component of Dynaslum in chapter 7.

\subsection{Discussion}

In this chapter we have provided an introduction to situation of slums in Bangalore using a novel on-field survey data from 37 slums. This chapter described the spatial, social, economic and environmental situation of slums in Bangalore. The demographic distributions, specifically, religion, language and migration to Bangalore were also studied using the survey data.

The slums in Bangalore are characterised by high level of poverty and low levels of education and skill training. Most slum dwellers are employed in the informal sector and do not have access to formal banking. Financial inclusion is at a low level with only $41 \%$ people have savings account in national banks. Loans and credit facilities from financial institutes are not accessible and around $49 \%$ slum households have borrowed from informal sources. Slum households spend the most on food and rent, however, the expenditure distribution on education is right skewed, where about $10 \%$ of the family spends more than INR 2000 on education. As far as security of tenure is concerned, we find that around $40 \%$ slum households have legal title to their dwellings.

Therefore, the contribution of this chapter in the design and implementation of Dynaslum is three-fold. First, the social, demographic and economic distributions highlights the heterogeneity in the slums of Bangalore which should be included in Dynaslum. Second, Dynaslum must include a robust residential choice model of slum dwellers through out their life cycle. Third, the survey data will be crucial in calibration and validation of different components in Dynaslum. In the following chapter 5, we further analyse segregation, income inequality and opportunity bias in the slums of Bangalore. 
4. SLUMS IN BANGALORE 


\section{Chapter 5}

\section{Multivariate Statistical Modelling of Segregation in the Slums of Bangalore.}

In India, religion, language and caste segregated human rights have long been alluded as a cause of inequality which in turn leads to exclusion and high degree of segregation among various social groups. However, increased urbanisation and economic growth in cities have diluted the effect of existing social structures such as caste, religion and language [89]. As individuals and households migrate into cities, prior societal norms fade away which may lead to less segregation [90, 91]. Furthermore, economic growth has helped reduce poverty in India, however the challenge is to distribute the benefits of growth uniformly over the diverse Indian socio-cultural and economic fabric. In India, urban poverty is spread unevenly among various social and ethnic groups and there is evidence that socio-economic based inequalities are less prevalent in more affluent parts of developed cities of India, while they are deep-rooted in second-tier cities and the urban poor of India [92]. In India, the socio-economic situation of individuals is inescapably correlated with their settlement, which leads to disparity in job prospects, healthcare and literacy. A recent survey on health and living condition in slums of eight Indian cities found that traditionally underprivileged castes disproportionately live in slums [93]. Similar quantitative studies on residential segregation in other cities of India also confirms the existence of high degree of segregation for the urban poor (slum dwellers) based on socio-economic status, language, caste and religion [94]. Thus, slums are distinct settlements, socially isolated from the rest of the city, primarily characterized by inadequate shelter, poor 


\section{MULTIVARIATE STATISTICAL MODELLING OF SEGREGATION IN THE SLUMS OF BANGALORE.}

access to basic services such as water and sanitation, insufficient access to healthcare and in general a low quality of life [4]. However, within such isolated settlements there are further dissociations based on religion, caste, language, occupation and other cultural differences which lead to a hierarchy of spatial patterns in slums [95, 96]. With respect to religion, Hindus dominate many slum areas as they are the majority. Muslims being the minority, segregate from other religious groups, while the population of Christians is usually low [95]. Besides religion, there are broad groupings based on caste, occupation and language. Thus, slums themselves have varying degree of inequalities isolating each group from the other. As a result, in-group connections are stronger and the socio-cultural interaction are limited within the group. It has been well established that spatial relations indicate the social distance between various groups and is one of the key channels of communication across individuals and social groups [97]. The association between socio-economic status and residential choice has been studied extensively in the past, especially in the context of developed countries. Similarly, studies in Indian cities show high level of segregation by income, education, occupation, caste and religion [97, 98, 99]. Recent studies have identified that conventional perception of slums as distinctive homogeneous settlements is incorrect, rather slums are diverse and complex systems that cannot be addressed through one-size fits all approaches $[4,100]$. A number of previous studies show that contextual differentiation for various types of slum settlements should be based on infrastructure, tenability and physical attributes $[101,102,103,104,105,106]$. However, these studies mainly focus on physical attributes and do not include the underlying spatial and socio-economic segregation of slum dwellers within each city.

In order to improve slum conditions, the spatial, social and economic characteristics of slums and the factors that shape them must be clearly understood. In this chapter we focus on getting a better understanding of the heterogeneity of slums. We examine the associations between groups of slum dwellers (based on religion, caste, language, income and occupation) to gain insight into how inequality and segregation are produced in slums. Spatial separation of these groups may fuel socio-economically bounded networks and in this way sustain categorical inequality [107]. Tilly calls this 'opportunity hoarding' or 'opportunity bias', a mechanism through which "members of a categorically bounded group acquire access to a resource that is valuable, reusable, subject to monopoly, supportive of group activities, and enhanced by the group's modus operandi" [108]. Therefore, the objective of this chapter is to understand how spatial and social segregation reproduce, or reinforce inequality and opportunity bias within 
the slums of Bangalore, an Indian city which has experienced high levels of urbanisation and rural-urban migration in the past decade.

This chapter draws on a field survey of 37 urban slums in Bangalore (described in the previous chapter) using a random stratified sampling with a target sample of 1100 households. The survey consisted of structured and open questions. Applying quantitative methods to the survey data (described in section 4.2), the chapter produces three key insights with respect to the social and spatial segregation within the slums of Bangalore. First, we find high levels of social and spatial segregation by religion and occupation in the slums of Bangalore. In each of these slums, residential segregation by religion is more prominent than the level of separation by socio-economic status. Second, we find that there is a high degree of inequality and opportunity bias towards certain sections of the society within the slums of Bangalore, which inhibits equitable access to jobs in the labour market. Finally, the results show that insufficient access to resources (such as, skill development, banking and finance) is a major hindrance for the slum dwellers to move out of poverty and constraints their social, spatial and economic mobility.

The chapter is organized as follows: section 5.1 provides a description of the methodology and the data. Section 5.2 presents the empirical analysis, based on the survey data, on occupations, income and religion and offers some preliminary explanations for the observed differences in the level of residential and economic segregation by religion and occupation. Finally, section 5.3 concludes with a discussion of the results and a way forward.

\subsection{Methodology}

In this section we elaborate the data, the theoretical framework and the quantitative methods employed to examine the segregation within the slums of Bangalore.

\subsubsection{Theoretical Framework}

In this section, we describe the application of Tilly's theory of "durable inequality" [108] to the survey dataset in order to gain insights into segregation and inequality across the slums in Bangalore. Tilly's theory identifies group segregation as the fulcrum of categorical inequality which further leads to opportunity hoarding. Tilly "argues that the same mechanisms generate and sustain group inequality, regardless of the content of group identities; whether these are defined by race, gender, religion, ethnicity, citizenship, tribe, caste, or class" [108]. Therefore, 


\section{MULTIVARIATE STATISTICAL MODELLING OF SEGREGATION IN THE SLUMS OF BANGALORE.}

understanding this "mechanism" in the context of slums in Bangalore can help in specific policy interventions that can reduce inequality and segregation.

In order to apply Tilly's theory in the context of Bangalore, we follow the following three steps. First, we use the Index of Dissimilarity to determine the key socio-economic categories (religion, language, gender, income and occupation) that leads to spatial and role segregation among the slums in Bangalore. Spatial segregation is analysed by the processes that assign groups to different social spaces across the city. Role segregation is analysed by the processes that assign groups to different social roles (such as different occupations). Second, to determine these processes for spatial and role segregation, we find association between the key social categories using Correspondence Analysis. Finally, we use Regression Analysis to show that the spatial and role segregation leads to socio-economic inequality and differentiated behaviour (such as fertility rates and gender preference) among various groups in slums of Bangalore.

\subsubsection{Quantitative Methods}

We employed the following concepts and multivariate statistical techniques to apply Tilly's theory in the context of Bangalore, discussed in section 5.1.1: Index of Dissimilarity, Correspondence Analysis and Regression Analysis, all further elaborated below.

\subsubsection{Index of Dissimilarity}

We calculated the spatial segregation across the 37 slums based on the five factors:- gender, religion, language, occupation and economic status using the index of dissimilarity (denoted by $D)[109,110]$. The Index $(D)$ is a measure of evenness, to quantify segregation and determine key socio-economic categories that drives segregation in the slums of Bangalore. It is computed as:

$$
D=\sum_{i=1}^{N}\left|\frac{Z_{i}}{G 1}-\frac{Z \iota_{i}}{G 2}\right|
$$

where $N$ is the total number of slums in the survey, $Z_{i}$ is the number of households belonging to group $G 1$ in slum $i, Z I_{i}$ is the number of households belonging to group $G 2$ in slum $i$. The index $(D)$ is useful to measure the segregation indices for two-group comparisons, such as the socio-cultural and economic variables of interest in our study. The five indicators used in this analysis: gender, religion, language, income group, and occupation category: split into two groups ( $G 1$ and $G 2)$ as shown in table 5.2. The formation of binary groups was done by creating 
all possible supergroups (see table 5.1) and identifying the combination which maximized the segregation .

\begin{tabular}{|l|l|l|}
\hline Ways to construct supergroups & 3 group & 4 group \\
\hline 1 & $(\mathrm{X}),(\mathrm{Y}+\mathrm{Z})$ & $(\mathrm{X}),(\mathrm{Y}+\mathrm{Z}+\mathrm{U})$ \\
\hline 2 & $(\mathrm{Y}),(\mathrm{X}+\mathrm{Z})$ & $(\mathrm{Y}),(\mathrm{X}+\mathrm{Z}+\mathrm{U})$ \\
\hline 3 & $(\mathrm{Z}),(\mathrm{X}+\mathrm{Y})$ & $(\mathrm{Z}),(\mathrm{Y}+\mathrm{X}+\mathrm{U})$ \\
\hline 4 & & $(\mathrm{U}),(\mathrm{Y}+\mathrm{Z}+\mathrm{X})$ \\
\hline 5 & & $(\mathrm{X}+\mathrm{Y}),(\mathrm{Z}+\mathrm{U})$ \\
\hline 6 & & $(\mathrm{X}+\mathrm{U}),(\mathrm{Z}+\mathrm{Y})$ \\
\hline 7 & & $(\mathrm{X}+\mathrm{Z}),(\mathrm{U}+\mathrm{Y})$ \\
\hline
\end{tabular}

Table 5.1: Formation of dichotomous groups.

Further, we calculated a reference point for the spatial segregation by calculating $D$ based on gender. The spatial separation by gender is expected to be very low within slums of Bangalore and provides a point of comparison for other socio-cultural factors of practical interest; in this case, religion, income and occupation based segregation. We then calculated spatial segregation by religion and various socio-economic indicators.

\subsubsection{Correspondence Analysis}

We use Correspondence Analysis (CA) to determine the association among the key indicators that drives spatial and role segregation (computed by $D$ ) in slums of Bangalore. CA [111] is similar to principal component analysis extracting eigenvectors, however it is better adapted for missing data which is typical for this kind of survey data. The associations between variables are uncovered by calculating the chi-square distance between different categories of the variables and between the individuals (or respondents). These associations are then represented graphically as "maps", which eases the interpretation of the structures in the data.Â In terms of the amount of variance accounted for, the first axis is the most important dimension, the second axis the second most important, and so on. The number of axes to be retained for analysis is determined by calculating eigenvalues $\mathrm{CA}$ is widely used in sociology $[112,113,114]$ and ecology for identifying associations among groups(or species) and focuses on relations between groups (or species) and their environment [115]. 


\section{MULTIVARIATE STATISTICAL MODELLING OF SEGREGATION IN THE SLUMS OF BANGALORE.}

\subsubsection{Stepwise Regression}

We use regression analysis to show that segregation leads to differentiated behaviour among various social groups in slums of Bangalore. Stepwise regression is applied to the fertility data to determine gender preference and mean live birth among different sections of the slum population.

\subsection{Results}

In this section we discuss the results of the dissimilarity index and CA to determine the association between religion, income and occupation and their impact on spatial segregation in slums of Bangalore.

\subsubsection{Degree of Segregation}

\begin{tabular}{|l|l|l|l|}
\hline Factors & G1 & G2 & D \\
\hline Gender & Male & Female & 0.07 \\
Language & Local & Foreign & 0.23 \\
Religion & Hindu & Muslim & 0.76 \\
Income & Low & High & 0.48 \\
Occupation & Formal & Informal & 0.64 \\
\hline
\end{tabular}

Table 5.2: Index of Dissimilarity

We calculated the dissimilarity index based on gender, religion, language, income group, and occupation category. Table 5.2 provides the value of $D$ based on the six factors for the slums in our study. We find that the degree of residential segregation by gender $(D=0.07)$ is small across the slums of Bangalore, however, religion $(D=0.76)$, income $(D=0.48)$ and occupation $(D=0.64)$ are the key factors that may drive segregation.

Segregation based on language was analysed by selecting each language as one group and comparing it with the rest of the languages as the other group. In this section we show the result by taking the local language (Kannada) in G1 and rest of all languages as G2, as this grouping produces the maximum value of $D$. Similarly, for income based segregation, G2 contains the high income group which is the top $32 \%$ or income group $6,7,8$ and 9 whereas G1 contains the bottom $68 \%$ or the income group 1 to 5 . In case of opportunity based segregation, we consider white and blue collar professionals as formal, who are primarily employed in the public sector (category 1 and 2) and rest as informal (category 3 to 12 ). 


\begin{tabular}{|l|l|l|l|l|l|}
\hline & Occupation & Income & Religion & Gender & Language \\
\hline Occupation & 1 & 0.84 & 0.85 & 0.28 & 0.87 \\
Income & & 1 & 0.86 & 0.21 & 0.73 \\
Religion & & & & 0.31 & 0.76 \\
Gender & & & & 1 & 0.56 \\
Language & & & & & 1 \\
\hline
\end{tabular}

Table 5.3: Correlation Matrix of Dissimilarity Index Based on 5 parameters.

Further, we calculate the correlations in $D$ based on the six factors as shown in table 5.3. The correlation matrix identifies underlying associations among these six factors. We observe high correlations in the value of $D$ among occupation, income, religion and language. To understand the underlying association we analyse the distribution of language among the religion. We find that most Muslims speak Urdu while the rest of the languages are distributed evenly among Hindus which suggests that any segregation based on Muslims would also reflect in population speaking Urdu. Therefore, to understand the actual segregation due to language we should exclude Urdu and understand segregation based on other languages. Similarly, occupation and income are correlated and analysing segregation patterns based on occupation should serve as a proxy for income.

\subsubsection{Spatial Segregation based on Religion}

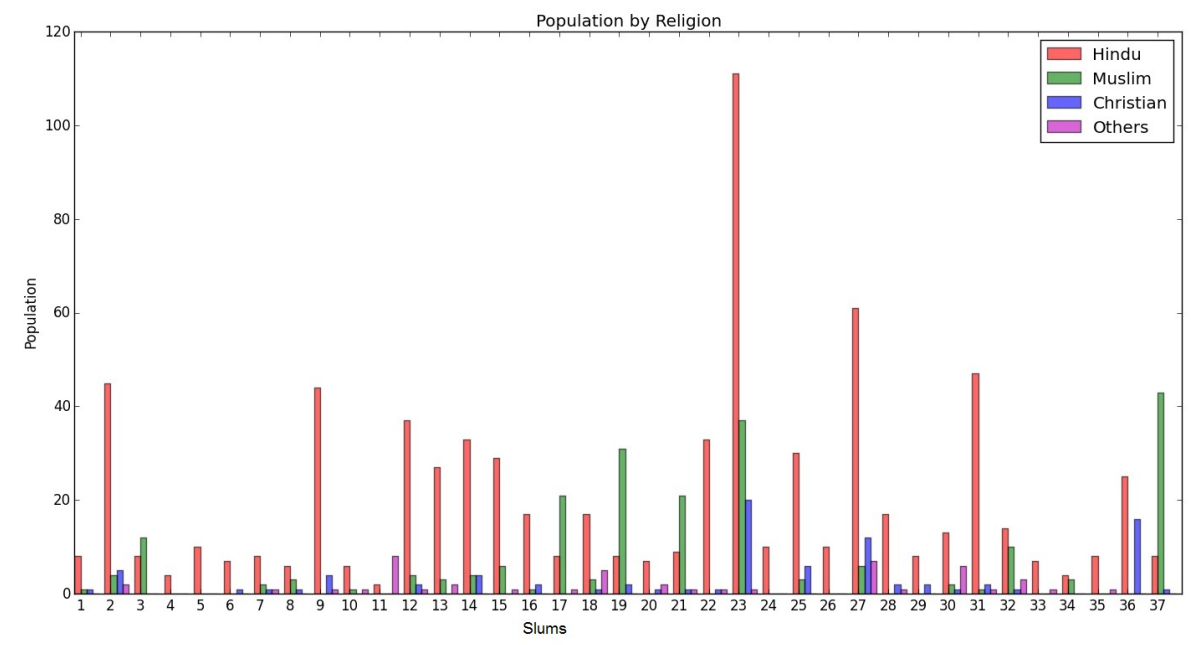

Figure 5.1: Religion Distribution across the 37 slums in Bangalore. 


\section{MULTIVARIATE STATISTICAL MODELLING OF SEGREGATION IN THE SLUMS OF BANGALORE.}

Slums in Bangalore are highly segregated based on religion as shown in section 5.2.1 and figure 5.2. CA was performed to determine the association of religion with the location of slums. Figure 5.2 shows that while Hindus are the majority and are spread across all the slums, the Muslims being a minority are segregated in the periphery of the city. This is clearly evident from the Muslim majority slums of Sadaramangala, Swatantrapura, Ashwath Katte and Hakki Pakki colony as shown in figure 5.1. The slums in the periphery, with an over representation of Muslim population, do also not have a 'right to the city', meaning that they are not notified. Spatial segregation can also be observed based on the language as shown in figure 5.3. It can be observed that slum households speaking Kannada (1) and Marathi (6) belong to one group. Similarly, most Muslim households speak Urdu (7) and are concentrated in few slums as mentioned above. The slum households speaking Tamil (3) are grouped in a different set of slums. Interesting slum households speaking Telugu (2) and Hindi (5) are flexible and do not show any language bias in their residential choice.

Slums can be classified based on their age. On one hand, the newer slums (10-30 years) have formed in the city's peripheral areas, such as near construction sites or the growing garment industry. On the other hand, the older slums are located in the core of the city, primarily home to the Hindu community, which have existed for 40-70 years (see table 5.4).

The residents of the older slums, have more deep-rooted links with political parties and local authorities as compared to the peripheral slums $[116,117]$. These notified slums have access to certain basic services provided by the local government, such as land tenure and public distribution system.

\begin{tabular}{|l|l|}
\hline Location & Average Age \\
\hline Core & 50.73 \\
Intermediate & 45 \\
Periphery & 26.94 \\
\hline
\end{tabular}

Table 5.4: Average Slum Age based on their Location

\subsubsection{Income Inequality and Poverty based on Religion}

Next to segregation, we also examined the association of income and social beliefs with respect to religion. Figure 5.2 shows that most Muslim slum households in the periphery of Bangalore usually occupied smaller dwellings as compared to slum households in the core of the city. Furthermore, figure 5.4 shows that the Muslims in slums of Bangalore experience a much 


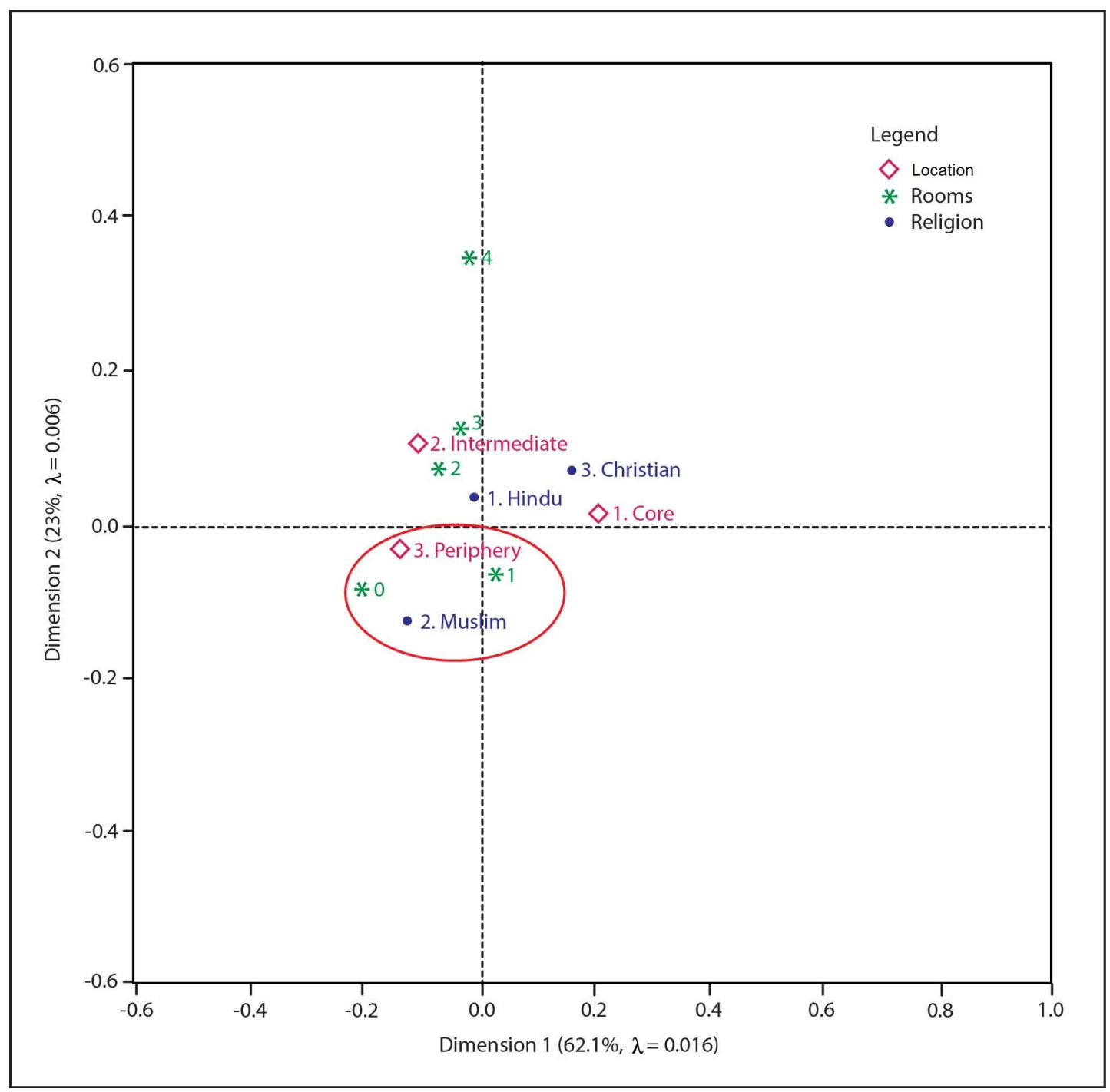

Figure 5.2: CA of religion, Number of rooms and location of slums.

higher incidence of poverty (lower income) than others. According to the survey data, almost $64 \%$ of all Muslims in the surveyed slums are below the 20th percentile in income levels as compared to $29 \%$ of the Hindus. The causes of higher poverty incidence among Muslims in the slums of Bangalore needs to be further explored. In India, poverty has been often associated with larger family size $[118,119,120]$. It is clearly evident from figure 5.4 that most Muslim households have larger families as compared to Hindus and Christians. The large family size among Muslim slum dwellers may be explained by the higher fertility rates (twice) as compared to Hindus and Christians. Stepwise regression analysis shows that per capita annual income 


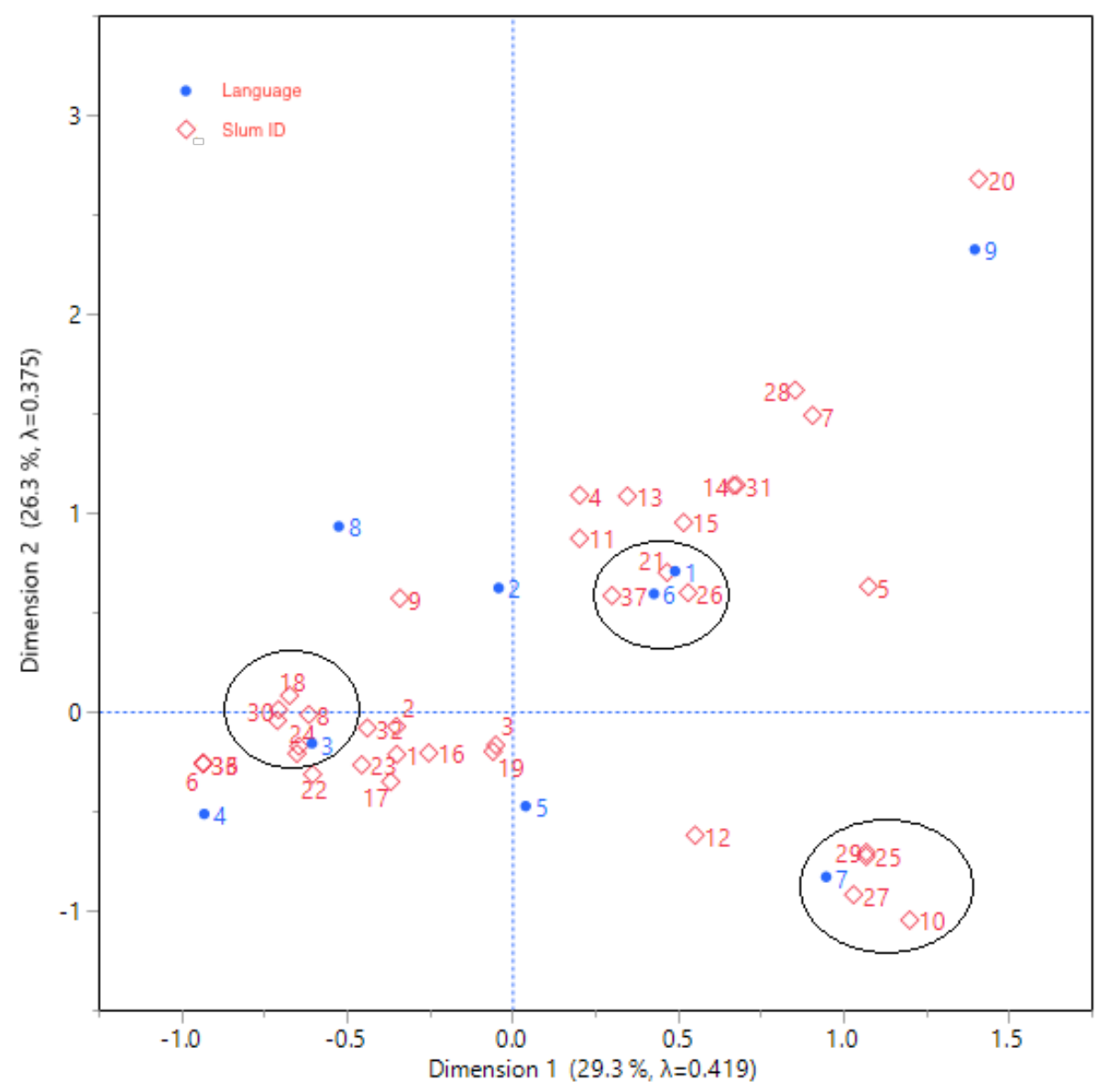

Figure 5.3: CA of language and list of slums in Bangalore. Language is categorised as (1 Kannada, 2 - Tamil, 3 - Malayalam, 4 - Telugu, 5 - Hindi, 6- English, 7 - Urdu, 8 - Bengali, 9 Others).

(PCAI), age, parity (number of births), and education have a significant influence on fertility among Muslim women in the slums of Bangalore. Regression analysis also clearly reflects that $47.5 \%$ of the total fertility is explained by these factors (table 5.5).

\begin{tabular}{|llll|}
\hline Factors & $\boldsymbol{R}$ square & Standard Error & $\mathrm{p}$-value \\
\hline PCAI & 0.164 & 1.93 & $0.0001^{*}$ \\
Age & 0.245 & 1.13 & $0.0013^{*}$ \\
Parity & 0.314 & 1.38 & $0.0001^{*}$ \\
Education & 0.475 & 1.85 & $0.0001^{*}$ \\
\hline
\end{tabular}

Table 5.5: Stepwise regression analysis of factors influencing fertility among Muslims in Slums of Bangalore. 


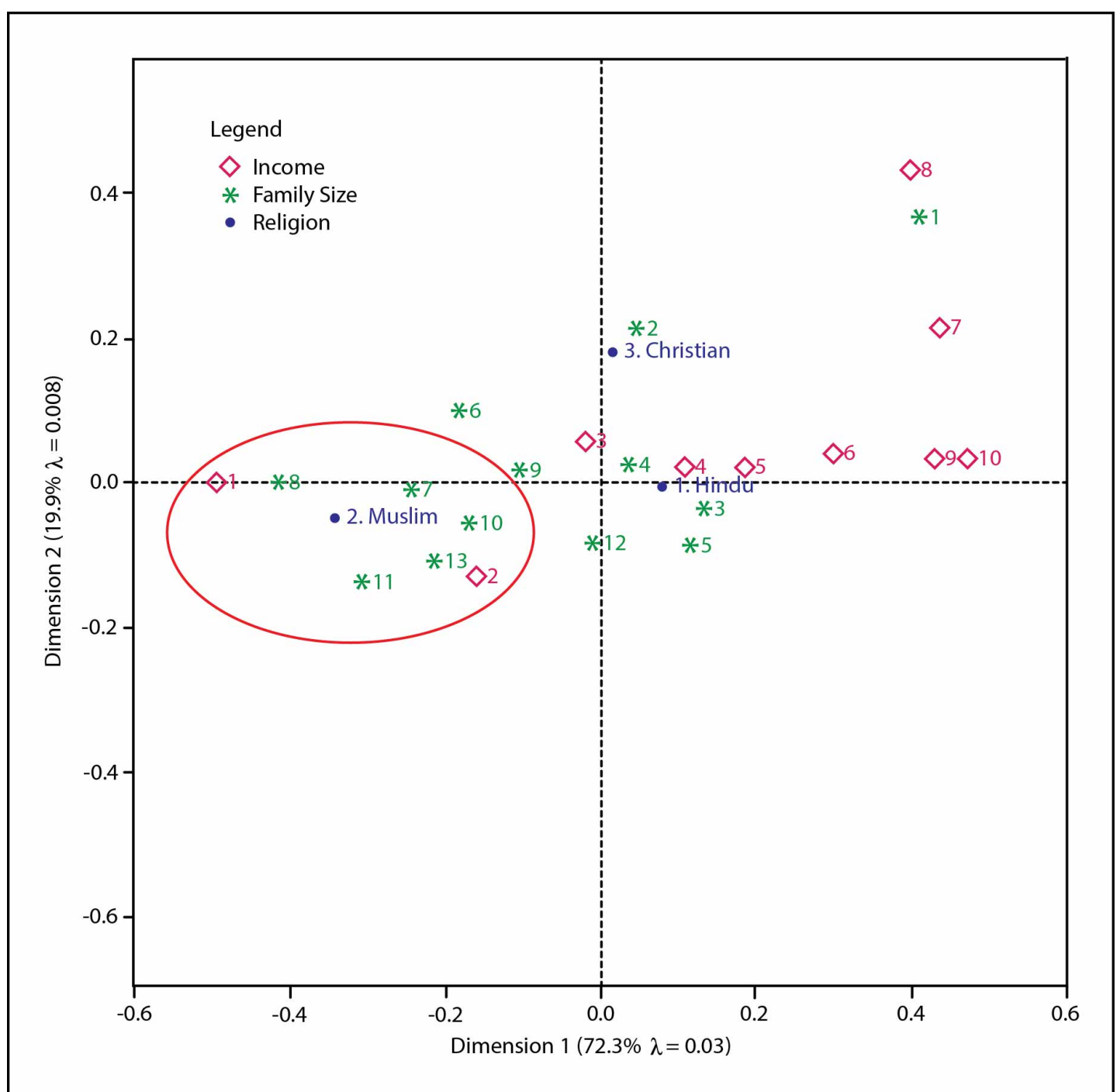

Figure 5.4: CA of religion, income and family size. Income is categorised as follows $(1-200$ to 1300, 2 - 1300 to 2400, 3 - 2400 to 3500, 4-3500 to 4600, 5 - 4600 to 5700, 6 - 5700 to 8000, 7 - 8000 to 12000, 8 - 12000 to 18000,9 - Greater than 18000 INR). Religion is indicated as follows (1 - Hinduism, 2- Muslim, 3-Christian, 4 - Others).

However, it is known that child's gender preference (GP) often leads to high fertility and therefore it is crucial to understand the link between GP and fertility rate [121]. It is observed from the survey data that the total number of children decreased with the increase in the birth of male child in first two live births. We have analysed the following three conditions:

1. First two children are male. 


\section{MULTIVARIATE STATISTICAL MODELLING OF SEGREGATION IN THE SLUMS OF BANGALORE.}

2. First two children are female.

3. First two children are mixed.

\begin{tabular}{|llll|}
\hline$N_{M}$ & $\mu_{B}$ & $N_{F}$ & $\mu_{B}$ \\
\hline 0 & 5.5 & 0 & 3.0 \\
1 & 5.0 & 1 & 5.0 \\
2 & 3.0 & 2 & 5.5 \\
\hline & & $r$ & p-value \\
\hline$N_{M}$ & & -0.134 & $0.001^{*}$ \\
$N_{F}$ & & 0.326 & $0.001^{*}$ \\
\hline
\end{tabular}

Table 5.6: Preference of male child and its association with fertility among Muslims in Slums of Bangalore. $N_{M}$ and $N_{F}$ are Number of male and female child in first two live births. $\mu_{B}$ is the Mean live birth. Pearson coefficient shows a negative correlation with the number of sons in first two live births with fertility, at the same time showing a positive correlation with number of female child in the first two live births

Table 5.6 shows that if a couple has two males as the first children, the total number of children decreased with the increase in the birth of male child in first two live births. The couples who do not have a single son in their first two births show the highest mean live birth (7.5). The mean live birth decreases to 5.0 when the couples have male children in the first two live births. The results shows that, in an effort to have a male child, many Muslim families continue to reproduce even after achieving their desired family size. Such differentiated behaviour means that local child policies such as "Bhagyalakshmi" [122] which encourages birth of female child, may not have desired effects on the Muslim community in the slums of Bangalore.

\subsubsection{Opportunity Bias based on Religion}

In this section we show the empirical analysis of slum occupations in Bangalore city in order to understand opportunity bias within the informal sector. Bangalore's slums contributed around $3 \%$ to the city's economy while housing $12 \%$ of the population in the year 2011 . In comparison, the slums in the neighbouring city of Chennai contribute around 14\% to the city's GDP each year while housing $19 \%$ of the total population [123]. The economic contribution of slums to the encompassing city's GDP is primarily determined by the nature of jobs and industries in the cities. The Bangalore's high skilled Information Technology industry is the largest employer in the city, but do not provide jobs to the slums dwellers, while in Chennai the low skilled labour intensive manufacturing industries provide jobs to a large number of slum dwellers [123]. 


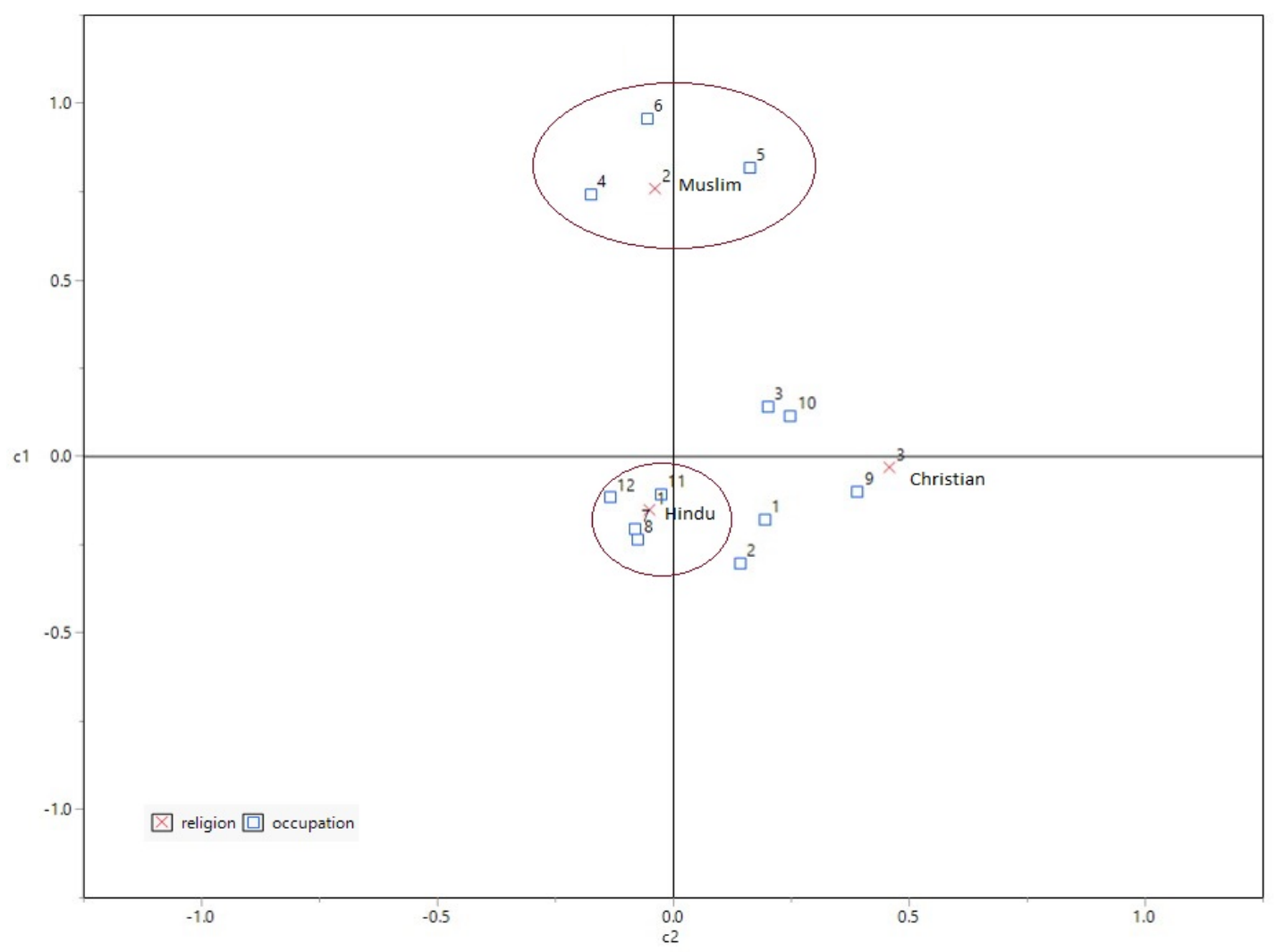

Figure 5.5: CA scatter plot of religion and occupation category (1 - Professional White Collar, 2- Professional Blue Collar, 3-Small Enterprises, 4-Self Employed - Sales, 5 - Self Employed Repair, 6 - Home Based, 7 - Domestic Services, 8 - Small Industry, 9 - Security, 10- Transport Services, 11 - Construction, 12 - manual labour).

\begin{tabular}{|l|l|}
\hline Source & Percentage \\
\hline Own sources & 37.29 \\
Informal borrowing(no interest) & 7.63 \\
Informal loan or money lender & 46.61 \\
Formal loan(without collateral) & 5.08 \\
Other & 3.39 \\
\hline
\end{tabular}

Table 5.7: Sources of Finance for Self-Employment

Occupationally, as shown in figure 5.6, large numbers of slum dwellers in the peripheral slums employed in the unskilled informal sector are predominantly self-employed, or work as construction workers. In contrast, the slum dwellers in the core of the city, work in a wider range of "organized" sectors, although characterized by low wages and lack of basic amenities. 


\section{MULTIVARIATE STATISTICAL MODELLING OF SEGREGATION IN THE SLUMS OF BANGALORE.}

Previous studies show that the median income in most informal sectors are far lesser than the formal jobs, restricting the ability of slum dwellers' to invest and mitigate future economic downturns $[124,116]$. Therefore, a low income coupled with lack of job security implies that the urban poor are not able to invest in education and housing, making it difficult for them to move out of poverty.

The informal sector provides a background for understanding the long-term, although everchanging, landscape of the capital-labour relationship in slums. In the case of slums in Bangalore, the present work draws attention to two significant sectors: first, industries like construction and manufacturing represent the growing and dynamic sectors linked to the local economy. Second, in the field of self-employment, trade and home-based employment provide daily sustenance for a large slum population. Figure 5.5 shows that job opportunities are highly segregated based on religion. A majority of Muslim slum households in Bangalore are engaged in self-employment, repair and services and home based manufacturing. However, these slumbased enterprises do not get any credit facilities from national or private financial institutions. Instead, they are forced to borrow capital from pawnbrokers who levy inflated interest rates, reducing growth and profit (table 5.7). Figure 5.4 shows that most Muslim households live in abject poverty and constitute a majority of the lowest income group in slums of Bangalore. Table 5.7 reveals that most slum-based businesses rely on middlemen to access the non-slum markets. These intermediaries receive a majority of the profits, further reducing the income of the owners and the employees. The majority of Hindu population, however, are employed in the formal sectors such as low income government jobs, construction and garment industries. Figure 5.6 shows the spatial distribution of each job category. It is evident that the average income of Muslim households is much lower than Hindus, indicating a deep-rooted opportunity bias based on religion. Therefore, it is important to understand and identify the unique characteristics of each group that largely comprise the informal sector, their specific vulnerabilities, and approaches to improve resilience of these groups. The majority of slum dwellers in Bangalore are low skilled and therefore cannot be absorbed by the development channels of Bangalore's economy, which is largely based on innovation and information technology. However, labour intensive sectors like manufacturing and construction still provides a minimum livelihood to large numbers of slum dwellers. In addition, the rate of growth of employment in the Small Scale Industries (SSI) sector has steadily declined from 14\% during 1971-80 to around 2.5\% in 2010-11, increasing unemployment among the urban-poor. 


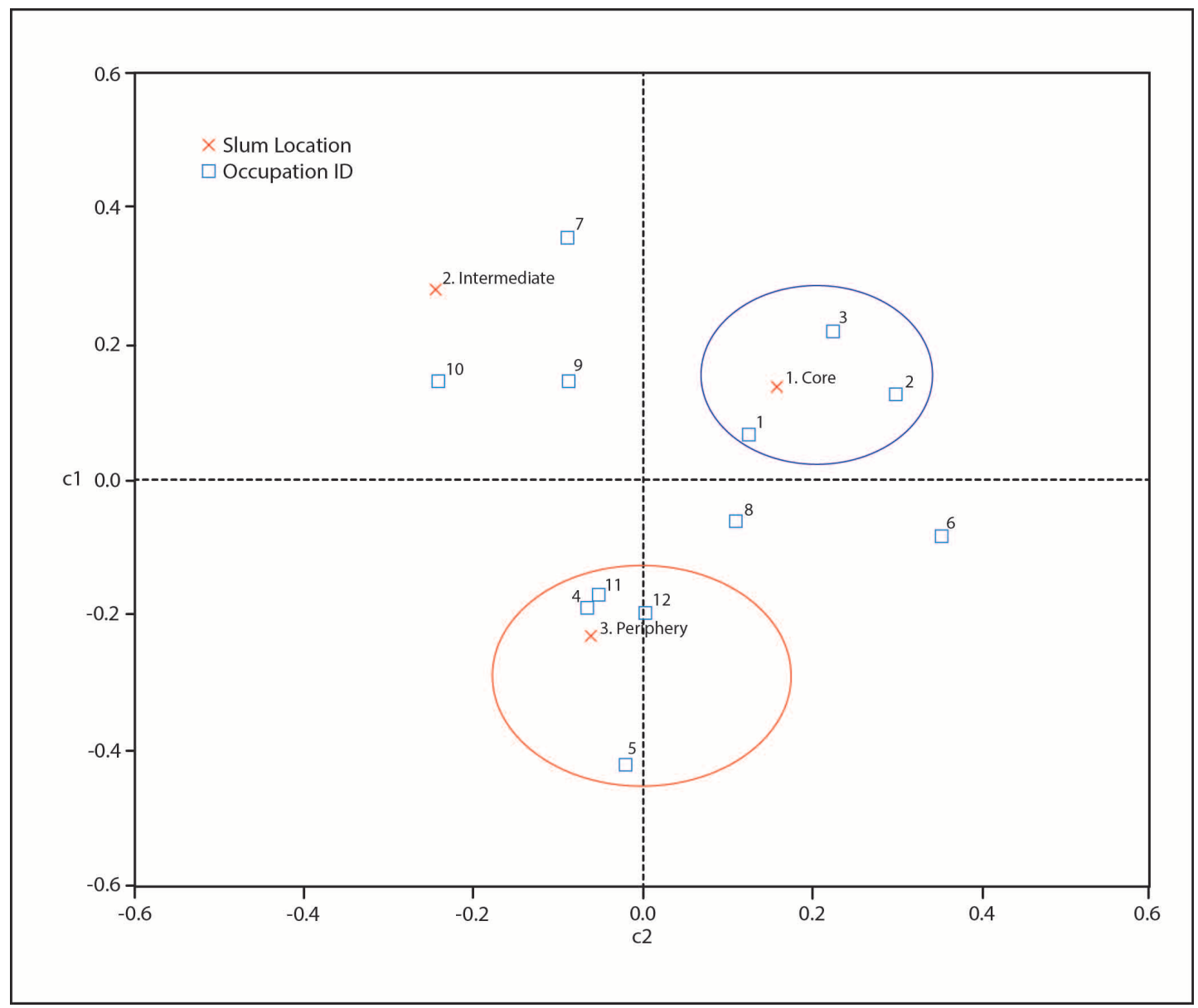

Figure 5.6: Spatial Distribution of Occupation Categories (1 - Professional White Collar, 2Professional Blue Collar, 3-Small Enterprises, 4-Self Employed - Sales, 5 - Self Employed Repair, 6 - Home Based, 7 - Domestic Services, 8 - Small Industry, 9 - Security, 10- Transport Services, 11 - Construction, 12 - manual labour).

Thus, the framework of the informal economy differs between different sectors, but the key attributes of informality - opportunity bias based on religion, unregulated incomes and high degree of financial insecurity remains a common feature across occupations in the informal sector. In this section, we have shown that spatial segregation based on religion leads to income inequality and opportunity bias, thus creating resource-rich and resource-poor social groups.

\subsubsection{Satisfaction Based on Religion}

In this section we show the result of CA between satisfaction levels of slum households and their religion. A value of 1 indicates a satisfied household, while a value of 2 denotes a dissatisfied 


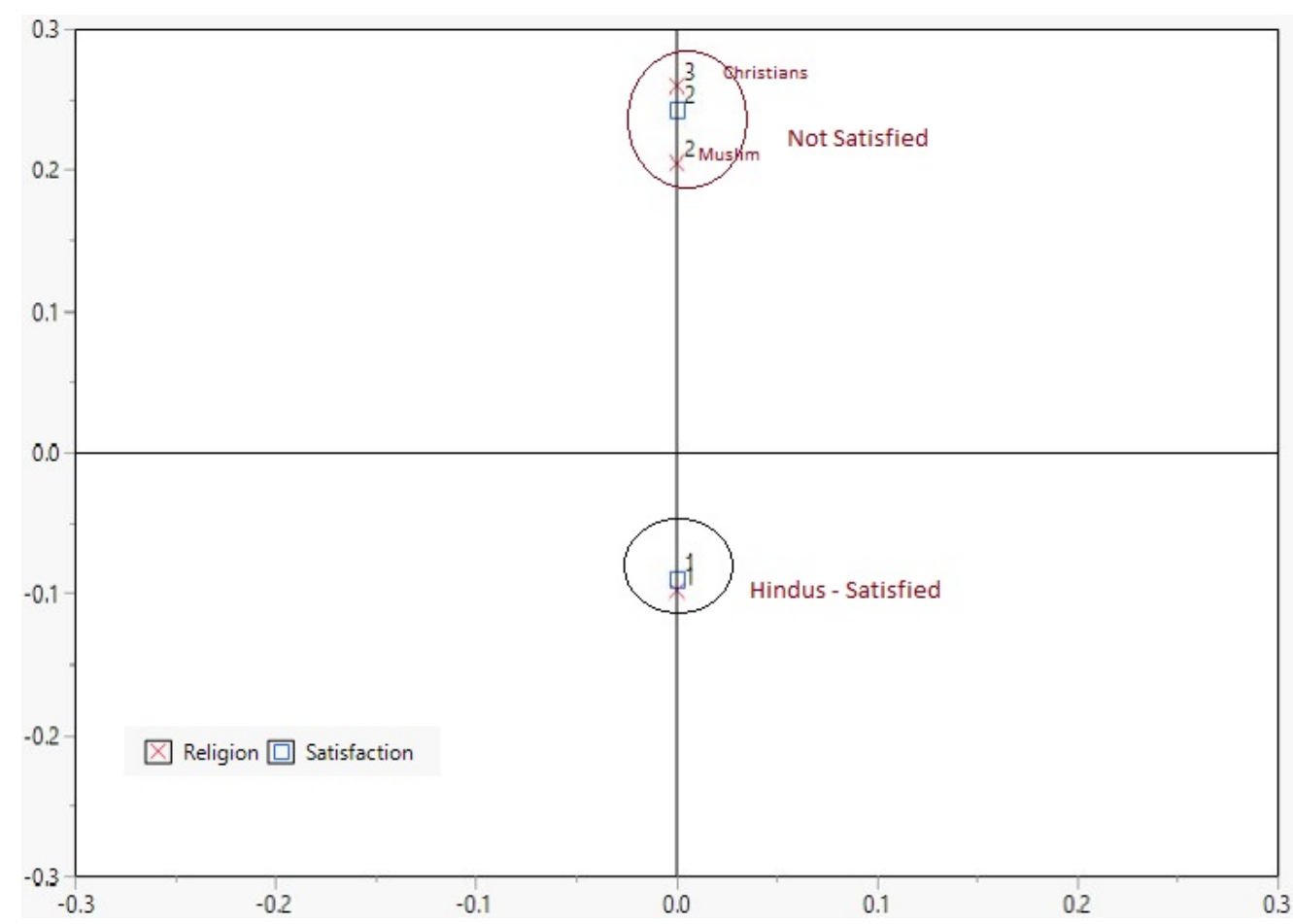

Figure 5.7: CA scatter plot of religion and satisfaction levels

household. Figure 5.7 shows that the majority of the minorities (Muslims and Christians) are not satisfied with their current socio-economic situation, while most of the Hindu households are satisfied.

As discussed in sections 5.2.2, 5.2.3 and 5.2.4 there is a strong evidence of group segregation and socio-economic inequality driven by religion in the slums of Bangalore. Muslim slum households are usually characterized by high fertility rates, large family size and low levels of income. Thus, the high degree of deprivation in the Muslim community is also reflected in the perceived satisfaction levels.

\subsection{Discussion}

In this chapter, based on unique survey data of 37 slums, we have shown that group segregation based on religion drives the formation of resource-rich and resource-poor social groups in slums of Bangalore. We find that spatial segregation and socio-economic inequality by religion dominates other factors (such as language, occupation). We also aimed at understanding whether spatial segregation is merely a manifestation of different socio-economic groups, or whether 
spatial segregation in itself has a role in producing segregated social groups. We disaggregate the effect of religion and income on the level of segregation, and find that income and occupation are highly associated with religion. In other words, should income or occupation alone determine the residential segregation of households, then the extent of segregation would have been much less. The findings also show that religious minorities, primarily Muslims are exposed to higher deprivation, opportunity bias and exclusion and have larger families and the lowest level of income. Our analysis shows that Muslim households have stronger preference for male children compared to other religious groups, which leads to higher fertility rates. Such segregated behaviour shows that child policies such as "Bhagyalakshmi" may not have the desired effects on Muslim households.

We have stratified the slum settlements for context-specific interventions based on socioeconomic and spatial segregation. Segregation of slums (based on religion and occupation) is an important component that needs to be deliberated while planning for effective slum redevelopment. We have shown that it is important to go beyond standard policies that are implemented in a top-down approach, which targets the physical environment without paying attention to segregated and marginalised groups of slum dwellers. Effective slum redevelopment may only be achieved if inclusive policies are formulated based on particular needs of slum dwellers. Further, if context specific policies are designed for each of the segregated group of slum dwellers it may increase the rate of successful interventions. It can also help policy makers and urban planners to think through different strategies for how to address the problems under differentiated context.

These findings have important implications for policy, urban planning and poverty alleviation in urban India, as new policies must consider diverse religious groups and dissimilar occupational skills that have proven critical to the survival of slums and their inhabitants. In addition to the above findings, the contribution of this chapter in the design and validation of Dynaslum is three-fold. First, we find that religion-based gender preference of the child at birth leads to increased fertility and impacts the household dynamics. Therefore, gender preference in reproductive behaviour must be included in Dynaslum. Second, we find that homophily is a dominant factor in spatial distribution and needs to be incorporated in the model. Finally, the index of dissimilarity $(D)$ represents the spatial segregation in slums of Bangalore and can be used in the validation of the model by comparing simulated value of $D$ with the actual. 
5. MULTIVARIATE STATISTICAL MODELLING OF SEGREGATION IN THE SLUMS OF BANGALORE. 


\section{Chapter 6}

\section{Population Dynamics Model}

In previous chapters we have shown that understanding the population dynamics and household life cycle is important as it is a crucial driver of residential mobility among slum dwellers. We have also identified that most existing growth models do not incorporate population dynamics in the model. In this chapter we describe the population dynamics model and discuss the key findings of the model. While slums differ in size and other characteristics from city to city, most slums are characterized by organic growth due to a high rate of rural-urban migration, high population density and low birth control measures. Amitabh Kundu argues that "the vision of slum-free Indian cities is hindered by poor urban planning and the failure to make adequate land and capital provisions for affordable housing". The challenge is to provide context-specific information to enable effective policy interventions. To design low-cost housing strategies, the policy makers and the urban planners need to have an understanding of how slum households make residential choices. In general, one of the most important factors accounting for relocation, has been a fundamental change in household composition and size throughout its life cycle. The individual life events such as marriage (and divorce), births and deaths, transition to adulthood and home leaving of adults may initiate a household's decision to relocate depending on the level of dissatisfaction from its current housing [125]. In chapter 2 we have shown, through the review of slum definitions and various slum policies, that it is important to incorporate household dynamics in any slum growth model. Further, in chapter 3 we have reviewed the existing slum growth simulation models and find that these models do not have a strong household dynamics component. In chapter 4 we provide empirical evidence and reasoning, from 37 slums in Bangalore, that household structure and composition plays an important role in the choice of slums. In this chapter we present the design and implementation of the household dynamics 


\section{POPULATION DYNAMICS MODEL}

component of Dynaslum and look into the dynamics of slum households through the lens of Household Life Cycle (HLC). This component is central to our key research question mentioned in section 1.3.

The analysis of HLC attempts to describe the effect of time on the household size through the individual life events and also helps to understand the behaviour of individuals in context which enables more effective interventions $[16,126]$. However, the HLC may vary across different sections of the society. It is therefore important to understand how the HLC of slum households evolves as compared to a non-slum households, as it will help explain at what stage of the HLC new households emerge from existing ones (or when they do not) and this in turn will indicate when new slums may emerge.

In the case of slums, the age structure, religion, language and composition of a household all play an important role in building the social network that may influence how slum dwellers settle in various slums in a city $[127,128]$. Therefore, understanding how the various social determinants grow and impact the behaviour of slum dwellers, and how they might influence slum households, can help us understand demographic processes and patterns of slum settlements and forecast how these settlement will change over time. In turn these insights may lead to effective low cost housing strategies. The individual life events such as marriage (and divorce), births and deaths, transition to adulthood and home leaving of adults may initiate a household's decision to relocate depending on the level of dissatisfaction from its current housing [125]. In the case of relocation of an entire slum, the collective movement involves households in different stages of their respective life cycles. Apart from the dynamics created by an individual's life events, the traditional composition of slum households have experienced dramatic changes over the past decade due to increased urbanization, improvements in health care, and changing social values $[129,130]$. The impact of such changes on the behaviour of slum dwellers are not yet well understood. Therefore, the key objectives of this chapter is to understand why slum households are different from non-slum urban households and identify the key factors that drive this difference.

In order to answer the above question, we adopt a computational approach to develop an ABM, namely the (Dynaslum), which can also be used for various What-If scenarios by policy makers. One common assumption included in most of the existing population models [131, 132] is that the structure and composition of a household does not change over time. Most population dynamics models have been developed and applied to different research domains (infectious disease, transportation) in the context of developed countries and may not be suitable for 
analysing slum settlements in developing countries because these models do not include the key cultural factors such as religion, language, origin and social beliefs (see section 6.1). Hence, it is important to capture the changing patterns of slum settlements as a result of the complex, multi-dimensional relationships that emanate from the HLC [133]. Dynaslum is designed to generate a synthetic population of slums and to describe household structure and composition in slums by including individual life events (see section 6.2). We choose India as our case study as it has experienced considerable urbanization and immigration of young adults from other parts of the country to engage in off-farm employment. Invoking theories and approaches from the social and computational sciences, Dynaslum seeks to understand relations between social and ecological systems and their associated effects on the HLC of slums. We use the city of Bangalore in India as a case to validate the demographic characteristics of Dynaslum. The city of Bangalore has $21.5 \%$ of the total slum population in the state of Karnataka, and every fifth person within the Bruhat Bangalore Mahanagara Palike (BBMP) limits lives in a slum. The population living in urban slums in Bangalore has doubled in a decade and this poses a serious challenge to urban planners and policy makers. The case is representative for a society in a developing country characterized by multiple religions and languages.

The remainder of this chapter is organized as follows. The literature review of existing population models is presented in section 6.1. The design of Dynaslum, execution steps and calibration of the model is discussed in section 6.2. The validation of Dynaslum and the sensitivity analysis with respect to HLC is discussed in section 6.3. In section 6.4, we present the key findings of Dynaslum and the difference in HLC between a slum and a non-slum household. Finally, the chapter concludes with a discussion on the findings and their implication.

\subsection{Related Work}

In the past, different methodologies have been developed for creating synthetic populations based on historical data in the field of healthcare, demography and social science. Understanding the impact of HLC on settlement choices are at the core of evolving research on population, land use, and the environment. Individuals or households as a social unit and migration as a component of population change have been central to population studies [134, 135, 136]. There is literature in ecology and geography that have addressed impacts of population on land use change and planning $[137,138,139]$. However, some approaches in ecology do not have 


\section{POPULATION DYNAMICS MODEL}

access to accurate data on population and are forced to make strong assumptions about human behaviour. Furthermore, while there are various approaches for population modelling and forecasting in demography $[140,141]$, studies on the impacts on land change and planning are scarce in that discipline [142]. In the past, various HLC models [17, 143] have been developed in the field of consumer behaviour based on age of the children and marital status of adult members of the household. The HLC models are based on a combination of casual empiricism and stereotypes to predict how spending patterns will vary across these households. Gilly and Enis [17] argue that earlier models had an overly inflated "other" category and do not incorporate a number of non-traditional household structures such as childless households, remarriage, middle-aged and older bachelors, never married or widowed single parents, and mature nest families. Various literature reflects differences of opinion on the usefulness of these HLC model and show that the models are not appropriate in dealing with non-traditional households [144].

Mathematical models have also been used to study demography by dividing population according to age or gender $[145,146,147,131,148]$. These models have also been proposed to incorporate household structure based on empirical distributions for household size. However, the household size distribution in these models do not evolve over time and therefore the models do not incorporate age structure and household compositions. Another methodology using modelling and simulation was used to study the impact of population dynamics on land use but the role of micro level processes was not included [4]. The state of population research has changed as more researchers are now actively involved in analysing population at the micro level using tools like ABM $[149,150,151,152,153]$. Many micro simulation models have also been developed for forecasting trend in population growth and corresponding policy interventions; they have been developed to understand past settlement patterns [130]. These models have adopted empirical methodologies to capture household composition. The initial population is generated using Monte Carlo method and subsequently updated every time step, using historical rates to estimate births and deaths, and assigning child birth based on empirical household size distribution. The household composition do not evolve over time and these models do not capture the impact of religion, language and origin. Despite the importance of population to slum settlements and land use change [154], population and household dynamics has not been central in slum research due to lack of accurate data at the appropriate scales and linkages. Most models discussed in this section have been developed and applied to different research domains and may not be suitable for analysing slum settlements in developing countries because these models do not include the key factors such as religion, language, origin, social beliefs and 
rural-urban migration, which is key to understand the slum dynamics in developing countries such as India. While we have drawn upon features from existing models [145, 150, 152] in the development of the population dynamics model, we observe that most of these models do not completely satisfy our objective of understanding life cycle of slum households. The elementary component of our model are the individual agents, characterized by their age, gender, language, religion and their current household.

\subsection{The Model}

This section outlines the design of population dynamics model. The idea is to take observed relations based on census data of India and the survey data described in previous chapter, combine them to calculate the demographic parameters and embed them in the model to simulate the population of slum dwellers in the city of Bangalore, India. Within the model there are two types of entities, the individual and the household. The individuals are the "agents" in the model, in that every individual is executed within a time step. However, agents are grouped into collections called households, influenced by the behaviour of household members and various demographic events. Households are not "agents" in that they are not executed, but each household is described through a number of attributes, e.g., household's age, religion, language, size. In the next section we describe the model according to the ODD (Overview, Design concepts, and Details) protocol [155].

\subsubsection{Overview}

\subsubsection{Purpose}

In this section we outline the objective of the population dynamics model in Dynaslum. The purpose of the model is to understand the observed differences between the household dynamics of slum and non-slum households and find the key factors that drives this difference. The model has been used to explore the minimum set of social and demographic events that are required to reproduce patterns that approximate the household size distribution and composition observed in real slums. Specifically, we look at the effect of home leaving among young adults and its impact on household life cycle of slum dwellers. The case study is representative for a society in a developing country characterized by multiple religions and languages.

Finally, the model is capable of generating a synthetic population which is representative of the city of Bangalore. In future, the model will be coupled with a residential choice model to 


\section{POPULATION DYNAMICS MODEL}

understand the relation between household life cycle changes and slum settlement patterns, as observed through remote sensing and parcel-based mapping.

\subsubsection{State Variables and Scales}

The model comprises of two types of entities, the individual and the household. The individuals are the "agents" in the model, in that every individual is executed within a time step. An individual is characterized by the state variables as listed in table C.1, Appendix C. However, agents are grouped into collections called households, influenced by the behaviour of household members and various demographic events. Households are not "agents" in that they are not executed, but each household is described through a number of attributes, e.g., household's age, religion, language, size. Table C.2, Appendix C lists the state variables of a household. Therefore, the model project agents individually, but in relation to each other, allowing for feedbacks over time.

\subsubsection{Process Overview and Scheduling}

The properties of the households and the individuals are updated at the beginning of a new time step, where each time step is currently equal to 365 days. The parameters influencing the occurrence of various demographic events are stated as annual probabilities and are scaled based on the duration of each time step.

The population size, structure, and distribution change in response to demographic and social events; namely births, deaths, migration, marriage and separation. The interplay between these events and population size and structure is essential for estimating both current and future slum population. Therefore, the state of an individual in Dynaslum can be updated by the following events at each time step.

- Mortality: Gender and age specific death rates (See figure 6.2 ) are derived to calculate an agent's (individual) possibility of dying during each time step. The age-specific death rate is defined as the number of deaths in a given year, per capita of the population in a particular age group. Since there are significant mortality variations between males and females, age-specific mortality rates are always calculated by gender. The differences in the age composition of populations are marginalized when age-specific death rates are used.

The death of an individual might lead to a household with only children, in that case the household is dispersed. Any adult child (i.e., aged over a specified year) can create a new 


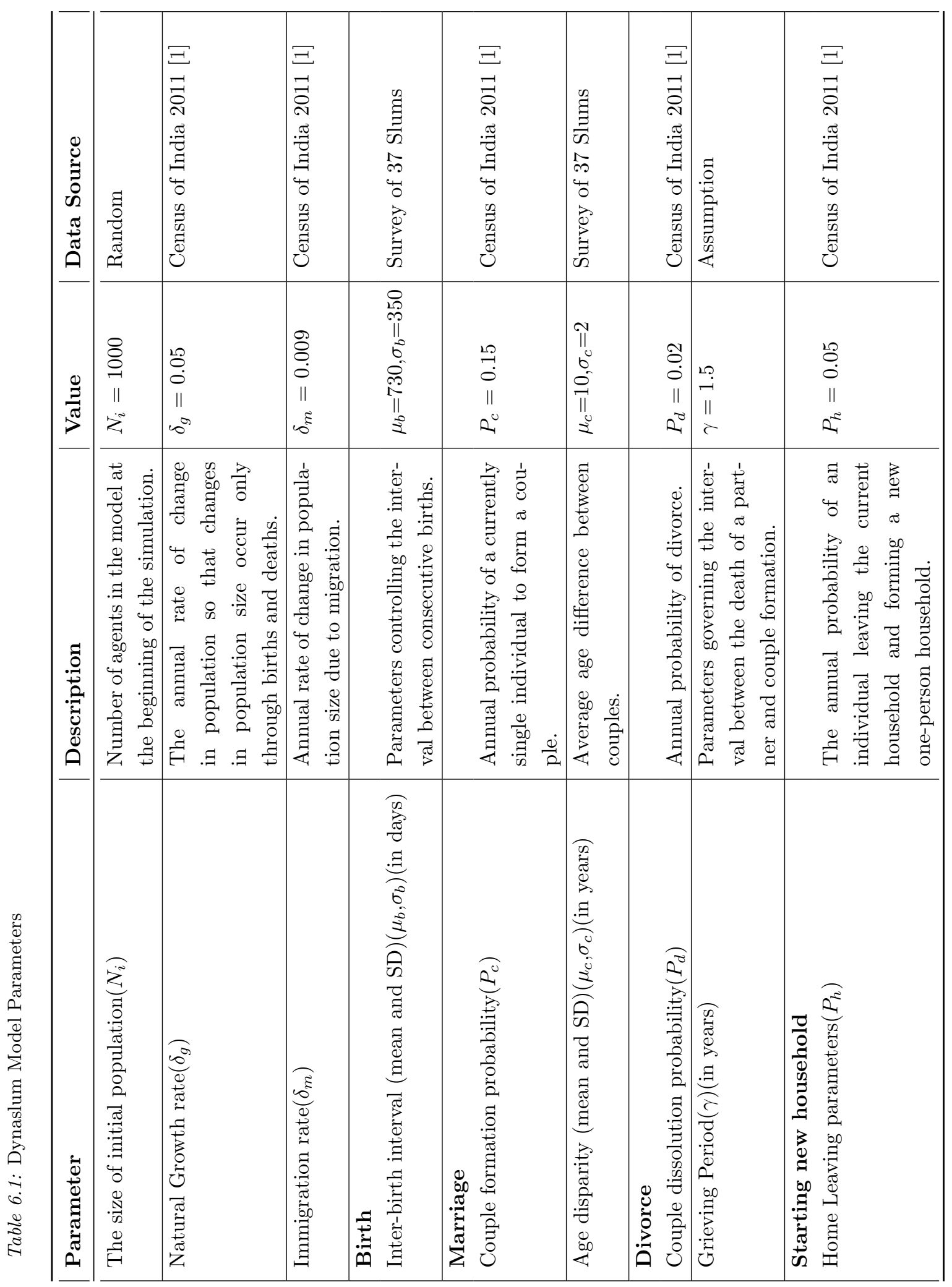




\section{POPULATION DYNAMICS MODEL}

household with the remaining household members. If the household does not have any adult child, they are all randomly assigned to other households which contain a couple and belongs to the same religion and language. If the death of an individual results in a household containing a widow or a widower it is then regarded as a special case of couple dissolution, which is discussed later in this section.

- Birth: Age and parity specific fertility rates (See figure 6.1) are derived to calculate the probability that a new agent that is born to a specific mother. The age-specific fertility rate denotes the number of live births per woman in a certain age group, per year. Differences in the age composition of populations are eliminated when using agespecific fertility rates. Given that a birth has occurred, probability distribution of age of the mother is generated from age-specific fertility rates, essentially by rescaling them. The gender of the new born is generated using a weighted random selection method based on the child sex ratio in Bangalore.

The age and parity specific fertility rates are used to determine the pool of eligible female individuals from which the mother is identified. Once a birth has occurred, the selected mother is excluded from any future births for a specified number of days derived from a normal distribution, with the minimum duration being 270 days. Therefore, the interbirth interval combined with the age and parity specific fertility rate controls the maximum number of children assigned to a mother.

- Couple formation: The individuals who are currently single undergo the process of couple formation $\left(P_{c}\right)$ with a given probability per time unit depending on their age range. The new spouse is chosen from the group of individuals from the same religion, speaking the same language, who are currently not coupled, belongs to the opposite gender, and whose age differs by a normally distributed value. The bride leaves her parent household to either form a new household with the groom or merge with the parent household of the groom depending on the household size, along with any dependent (e.g., dependent from past marriage).

The model assumes that the probability of couple formation is independent of gender, previous marital status, dependants, income, and other important factors due to lack of quality data from slums. The incidence of inter religion and inter state marriages are very rare in slums of Bangalore [156] and is not included in the model. The model also assumes that the probability of remarriage is the same as new couple formation and ignores the 
effect of religion, dependent, income and other important factors. This may be a fair assumption as the incidence of remarriage is high in slums [157]. However, the motivation of adapting this simpler approach originates from the lack of quality data with respect to marriage in slums.

- Couple dissolution: The individuals who are currently coupled undergo the process of dissolution with a given probability $\left(P_{d}\right)$ per time unit depending on their age range. The process of dissolution leads to the division of the existing household, with male member moving out of the household to form a new one-person household, whereas the other member continues as a part of the original households along with possible dependents. The separated individuals are then eligible to remarry as per the process mentioned under couple formation.

In case dissolution results from the death of a partner, the widow or the widower has to wait for a specified amount of time (known as grieving period) before they are eligible to remarry.

- Leaving home: Each individual gets separated from their household when they get married and may start a new household with their spouse as described under couple formation. Otherwise, an individual may also move out of their household with a certain probability per time step, once they reach a specified age.

- Immigration: The immigrant population are treated as household units in the model, with their household size and the household composition drawn from an empirical immigrant population distribution. As many as $90.61 \%$ of slum dwellers in Bangalore are the natives of Karnataka state. About $5 \%$ of them reported that their native state is Andhra Pradesh while the remaining $4.33 \%$ migrated from Tamil Nadu [1].

\subsubsection{Design Concepts}

\subsubsection{Emergence}

The population dynamics model is designed to understand the interaction between demographic events and household life cycle. The household structure and composition are emergent as they develop due to the interaction between patterns of household creation and termination arising from various demographic events. Therefore, the household life cycle are not drawn from a distribution but evolve in the model. 


\section{POPULATION DYNAMICS MODEL}

\subsubsection{Adaptation}

In population dynamics model, the agents adapt their state through the 6 stochastic events during each time step as discussed in section 6.2.1.3. The adaptation is based on the current situation of the agents and the model parameters (described in table 6.1). This enables the model to reproduce convincing distribution of household structure, which emerges from the interactions among various social and demographic events. Further, the model generates useful data on family relationships among agents that may be used to create multi-level extended family networks. The present model can be easily adapted to study disease spread, land uses, development of migration strategies and understand critical behaviour related to the residential choice of slum dwellers.

\subsubsection{Fitness}

In the model, the gender of each birth is based on assigned preferences when they are created. It is observed from the survey data that the total number of children decreased with the increase in the birth of male child in first two live births as shown in previous chapter (5.6)

\subsubsection{Prediction}

Dynaslum is not a predictive model. The primary objective is to analyse the underlying factors that drives the difference in population dynamics between slum households and non-slum formal households. However, the agents in the model can forecast their state in the following two situations. First, during birth, the selected mother cannot be assigned any children for specified time steps (described in section 6.2.1.3). Second, in case dissolution results from the death of a partner, the widow or the widower has to wait for a specified amount of time (known as grieving period) before they are eligible to remarry (described in section 6.2.1.3).

\subsubsection{Interaction}

Agents interact directly when an couple formation takes place. The couple may form a new household as discussed in section 6.2.1.3.

\subsubsection{Sensing}

Sensing is not implemented in the model. 


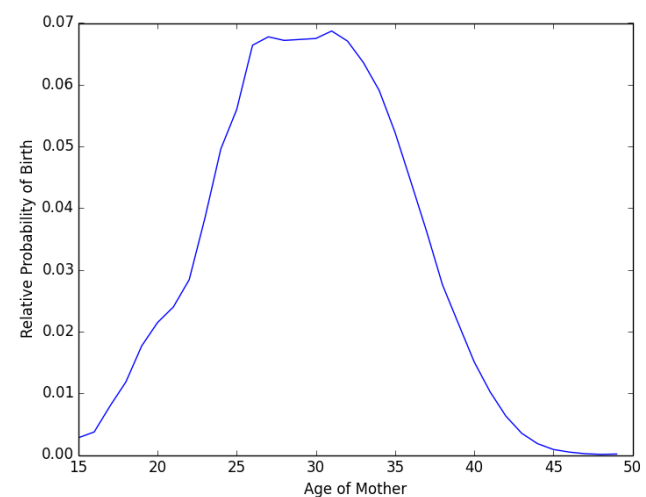

Figure 6.1: Relative probability of birth at a given age [1].

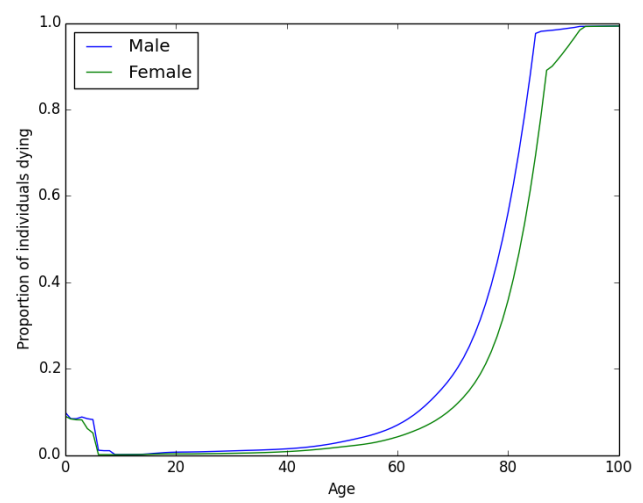

Figure 6.2: Annual risk of death by age and $\operatorname{sex}[1]$.

\subsubsection{Stochasticity}

The model parameters that have been used to generate the initial population and update the population are summarized in table 6.1. The model is calibrated using data derived from demographic metrics listed in various surveys and census data of India. A model calibration process is used to find a best fit set of parameter values assigned to agents' preferences for different demographic events (marriage, home leaving, and divorce). The parameters in the model are stochastic and control the social and demographic events that occur to the individuals, which leads to the formation of different sizes and types of slum households. For instance, each birth was assigned to their respective mother based on the relative probabilities, but not to fit a particular household size distribution. Thus, contrary to most existing population models, household size and composition is an emergent phenomena in the model.

\subsubsection{Collectives}

Each agent is associated to a household. New households are formed due to couple formation, couple dissolution, home leaving and immigration of new households.

\subsubsection{Observation}

Observation include capturing the household structure and composition based on the demographic events which in turn leads to the household life cycle Household life cycle is the measured output of the model and is used to perform the sensitivity analysis and the validation. 


\begin{tabular}{|r|r|}
\hline Age & Percentage \\
\hline Below 15 & 11.00 \\
15 to 25 & 16.00 \\
25 to 35 & 23.33 \\
35 to 50 & 33.33 \\
51 and above & 16.33 \\
\hline
\end{tabular}

Table 6.2: Age Distribution.

\begin{tabular}{|r|r|}
\hline Language & Percentage \\
\hline Kannada & 56.67 \\
Telugu & 13.67 \\
Tamil & 26.33 \\
Others & 3.33 \\
\hline
\end{tabular}

Table 6.4: Language Distribution.

\begin{tabular}{|r|r|}
\hline Religion & Percentage \\
\hline Hindu & 81.67 \\
Muslim & 9.00 \\
Christian & 8.33 \\
Others & 1.00 \\
\hline
\end{tabular}

Table 6.3: Religion Distribution.

\begin{tabular}{|r|r|}
\hline Household Size & Percentage \\
\hline Less than 3 & 19.00 \\
3 to 5 & 15.00 \\
6 to 7 & 64.33 \\
8 and above & 1.67 \\
\hline
\end{tabular}

Table 6.5: Household Size Distribution.

\subsubsection{Details}

\subsubsection{Input}

In this section we describe the four key distributions (derived from Census of India [1]) that are used to assign individuals to households while creating the initial population. The model depends on the following input data to generate a realistic pattern of household structure and composition in the initial population.

1. Age Distribution - It denotes the proportion of individuals of a given age in the population at a given point in time (see table 6.2 ).

2. Religion Distribution - It represents the proportion of individuals in the slum population of Bangalore who belong to one of the four main religious categories, namely, Hinduism, Muslim, Christianity, and Others (see table 6.3) .

3. Language Distribution - It represents the proportion of slum dwellers speaking one of the five major languages, namely, Kannada, Telugu, Tamil, Hindi, and Others (see table $6.4)$.

4. Household Distribution - The household size distribution denote the distribution of households by size of household as given in table 6.5 . 


\subsubsection{Initialization}

The first step in generating a synthetic population is to create an initial population, which accurately represents the population distribution of slums in the city of Bangalore. We generate the initial bootstrapped population by randomly creating agents with their ages derived from the age distribution (see table 6.2) of slums in Bangalore. The gender of the initial population is generated using a weighted random selection method based on the sex ratio in Bangalore. These initial agents (or individuals) are then randomly structured into households based on the household size distribution (see table 6.5) of the city. The households with one or two members are allocated one or two adults from the initial population, while households with three or more members are allocated two adults and one or more children. Once households are created, they are assigned different religions based on the religion distribution (see table 6.3). The final step of the initialization process assigns a language to each household based on the distribution drawn from language distribution data (see table 6.4 ). This process of creating initial population may not satisfy real-world constraints such as interval between birth and age difference between family members. Therefore, we adopt an approach to run the model until (warm-up period of 100 time steps) the simulated population has replaced all the initial households, at which stage the above constraints will be satisfied.

\subsubsection{Calibration of Dynaslum}

The model parameters that have been used to generate the initial population and update the population are summarized in table 6.1. The model is calibrated using data derived from demographic metrics listed in various surveys and census data of India. A model calibration process is used to find a best fit set of parameter values assigned to agents' preferences for different demographic events (marriage, home leaving, and divorce). The parameters in the model control the social and demographic events that occur to the individuals, which leads to the formation of different sizes and types of slum households, emerging as a result of these micro level social and demographic events. In case of our model, each birth was assigned to their respective mother based on the age-parity-specific fertility rates, independent of the household size distribution, meaning not to a particular household which would control the size and composition. Thus, contrary to most existing population models, household size and composition emerged from the interaction between various social and demographic events. We describe the calibration strategy (discussed in table 6.1) for each parameters below: 


\section{POPULATION DYNAMICS MODEL}

- Natural Growth $\operatorname{rate}\left(\delta_{g}\right)$ : The natural growth rate is estimated by compounded annual growth rate (CAGR) from the decade growth rate between 1991 to 2001 and 2001 to 2011 from the Census of India 2011.

- Immigration rate $\left(\delta_{m}\right)$ : The immigration rate is estimated by compounded annual growth rate (CAGR) from the decade growth rate between 1991 to 2001 and 2001 to 2011 from the Census of India 2011.

- Inter-birth interval (mean and $\mathrm{SD})\left(\mu_{b}, \sigma_{b}\right)$ (in days): The inter-birth interval (mean and standard deviation) is estimated using the survey data from 37 slums in Bangalore.

- Couple formation probability $\left(P_{c}\right)$ : The couple formation probability is calibrated to fit the 2001 data from the census of India 2001.

- Age disparity (mean and $\mathrm{SD})\left(\mu_{c}, \sigma_{c}\right)$ (in years): The inter-birth interval (mean and standard deviation) is estimated using the survey data from 37 slums in Bangalore.

- Couple dissolution probability $\left(P_{d}\right)$ : The couple dissolution probability is calibrated to fit the 2001 data from the census of India 2001.

- Grieving $\operatorname{Period}(\gamma)($ in years): This is an assumed value.

- Home Leaving parameters $\left(P_{h}\right)$ : The home leaving probability is calibrated to fit the 2001 data from the census of India 2001.

\subsection{Validation and Sensitivity Analysis}

In this section, we use the case study of the city of Bangalore in India to validate Population Dynamics Model. We also highlight the differences between slum and non-slum households and identify key parameters that drives this difference.

Quantitative comparison between simulation output and real world historical data is a significant element of the validation process. However, in a developing country scenario, accurate longitudinal data for validation is scarce; hence we also depend on qualitative knowledge from slum literature to validate the model $[83,152]$. Populations in slums change rapidly and are usually characterized by higher rate of migration and higher household size compared to the formal parts of the city. In the case of population dynamics model, we have analysed a situation in which parameters such as age specific death rates, age specific fertility rates, couple 


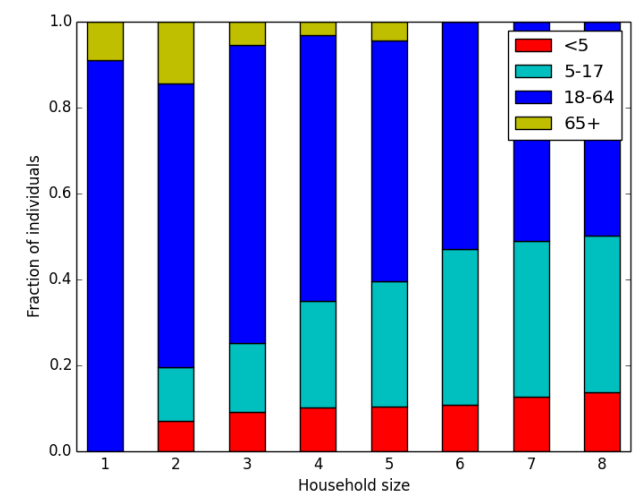

Figure 6.3: Household Age.

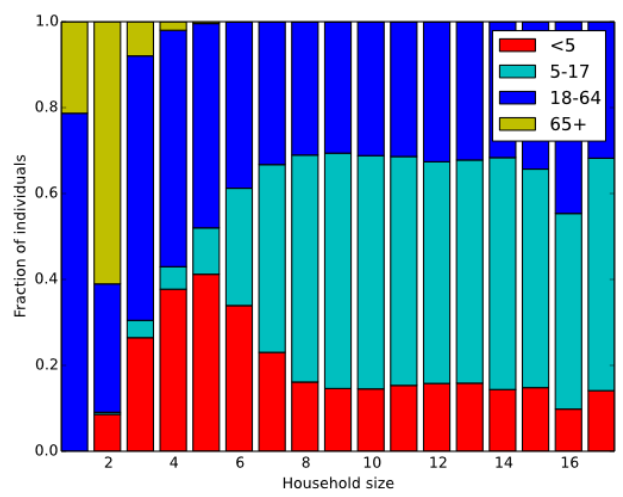

Figure 6.4: Household Size.

formation probabilities and couple dissolution probabilities are constant, and are calculated on the basis of past data on marriage and divorce amongst the urban-poor in the city of Bangalore $[14]$.

\subsubsection{Validation of Model Initialisation}

In this section we validate the model initialisation methodology. The model was initialised and executed for a warm up period of 100 time steps (years), which corresponds to the year 2001 in the simulation. The model was further executed for another 10 time steps (years) to project the population in year 2011. The resulting population (averaged over 100 runs) demonstrated that key population trends were replicated in the synthetic populations generated by the population dynamics model. Table 6.6 shows that the size of total simulated slum population in Bangalore is comparable to that observed in the actual data.

\begin{tabular}{lllll}
\hline Year & $N_{\text {Act }}$ & $N_{\text {Sim }}(\mathrm{SD})$ & $\varphi_{\text {Act }}$ & $\varphi_{\text {Sim }}(\mathrm{SD})$ \\
\hline $\mathbf{2 0 0 1}$ & 345,200 & $340,335(510)$ & 1.052 & $1.046(0.001)$ \\
$\mathbf{2 0 1 1}$ & 712,801 & $700,614(824)$ & 1.035 & $1.033(0.0009)$ \\
\hline
\end{tabular}

Table 6.6: Simulated slum population (averaged over 100 runs) vs Actual slum population in Bangalore. $N_{\text {Act }}$ and $N_{\text {Sim }}$ represents the total actual and simulated slum population in Bangalore. $\varphi_{\text {Act }}$ and $\varphi_{\text {Sim }}$ represents the actual and simulated sex ratio (male to female ratio) in slums of Bangalore. The standard deviation (SD) is also shown in parenthesis 


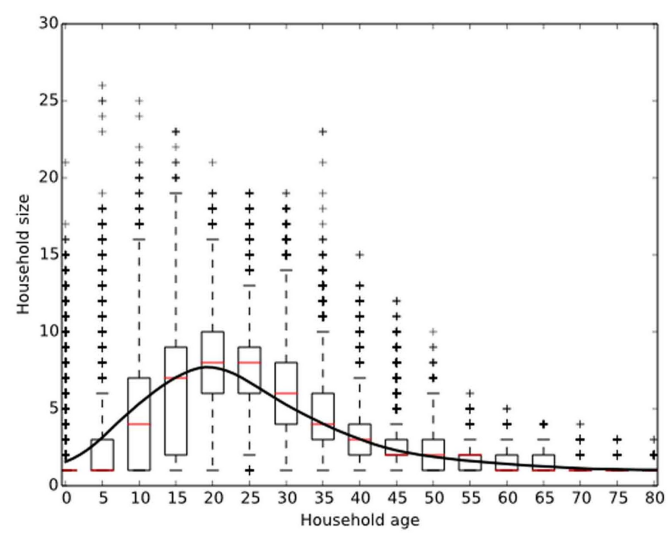

Figure 6.5: Simulated HLC of slums in Dynaslum.

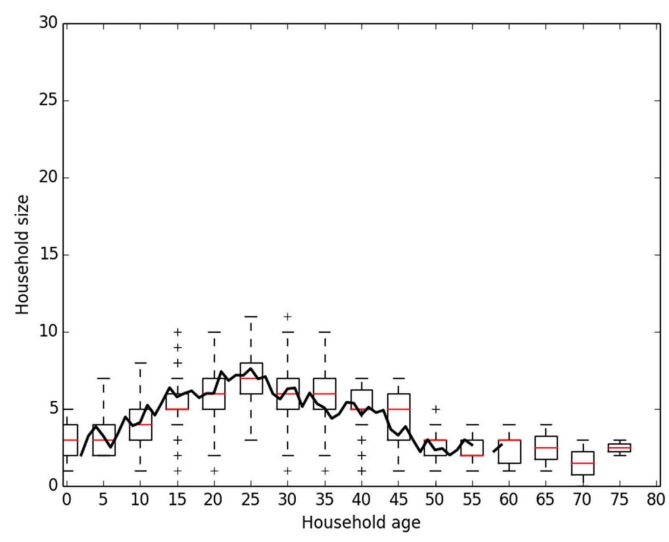

Figure 6.6: Actual HLC from real data of slums in Karnataka, year 2005 [2].

\subsubsection{Validation of Model Steps}

In this section we validate the household size, composition, and HLC in response to various demographic events. The household composition has been categorized by separating individuals into distinct age groups relevant for settlement choices in slums: infants under the age of five years; children between 5 and 17 years; adults from 18 to 64 years; and elderly individuals 65 years and over. Figures 6.4 denote the distribution of fraction of individuals of a given age group based on the household size at a particular time step in the simulation. These patterns are comparable to the observed trends of household composition (6.3) in various slums across the city of Bangalore. Individuals over the age of sixty-five years are commonly found in smaller and older households, while children between the age of five and seventeen years are found mostly in households of intermediate age.

As individuals move in and out of a household to start new households the size of the household constantly changes. The similarity in the HLC patterns in figure 6.5 and figure 6.6 confirms that Dynaslum is able to produce realistic HLC of slums in Bangalore.

\subsubsection{Sensitivity Analysis}

In this section, we perform global and local sensitivity analysis of the model described in section 6.2 and identify parameters that have the most influence on the behaviour of the HLC. The household size was captured at various household ages $(0,20,40,60$, max $)$ to determine if a change in parameter value can change the HLC pattern. 


\begin{tabular}{|l|l|l|}
\hline Parameter & Nominal Value & Range \\
\hline$\delta_{g}$ & 0.05 & $0.01-0.08$ \\
$\delta_{m}$ & 0.009 & $0.01-0.08$ \\
$P_{c}$ & 0.075 & $0.1-0.8$ \\
$P_{h}$ & 0.05 & $0.01-0.09$ \\
\hline
\end{tabular}

Table 6.7: Model parameters and range for sensitivity analysis

\subsubsection{Sobol' Indices}

In order to perform the global sensitivity of the model, we compute the Sobolo' first order $\left(S_{1}\right)$ and total-order sensitivity $\left(S_{t}\right)$ indices using Saltelli's sampling method [158] with 100 model runs uniformly distributed in the range shown in table 6.7 [159]. $S_{1}$ accounts for both linear and non-linear contributions of individual parameters but no interaction effects, while $S_{t}$ includes the interaction effects of the parameters. The output of the model for calculating the Sobol' indices is measured as the difference between peak household size in the life cycle and household size at 40 . The resulting $S_{1}$ and $S_{t}$ are shown in table 6.8 , where the accuracy of the estimate sensitivities are derived based on bootstrap confidence intervals $\left(S_{1 \text { conf }}\right.$ and $\left.S_{\text {tconf }}\right)$ [160]. Table 6.8 shows that sum of all the first order indices equals to 0.76 which indicates that the contribution of higher order interactions is around 0.24 which is much less compared to the first order contribution.

\begin{tabular}{|l|l|l|l|l|}
\hline Parameters & $S_{1}$ & $S_{1 \text { conf }}$ & $S_{t}$ & $S_{\text {tconf }}$ \\
\hline$\delta_{g}$ & 0.10 & 0.1892 & 0.12 & 0.186072 \\
$\delta_{m}$ & 0.02 & 0.0038 & 0.08 & 0.032064 \\
$P_{c}$ & 0.31 & 0.10381 & 0.37 & 0.167723 \\
$P_{h}$ & 0.33 & 0.17662 & 0.43 & 0.157856 \\
\hline
\end{tabular}

Table 6.8: First order and total-order Sobol' sensitivity indices

Therefore, using Sobol's analysis we conclude that $P_{c}$ and $P_{h}$ are the most sensitive parameters with respect to the household life cycle patterns. However, this method considers only the mean effect of parameters and does not capture the patterns in the parameter space. Since, the interaction effects are marginal in our case, we perform a One Factor at a time (OFAT) analysis to see the robustness of patterns to individual parameter changes. 


\section{POPULATION DYNAMICS MODEL}

\subsubsection{One Factor At a Time (OFAT)}

OFAT sensitivity analysis basically functions on a base parameter setting (nominal set) and then changing one parameter at a time while keeping all other parameters constant (hence it is referred to as a local sensitivity analysis). Therefore, in this section we vary each parameter over the parameter space and plot the resulting output. In the first set of experiments, we analyse the sensitivity of growth rate while keeping all other parameters constant described in table 6.1. The model was run 100 times for each parameter setting and the average was calculated to compare the results. Figure 6.8 shows that as the growth rate increases, the household size also increases. However, the HLC continues to follow an ideal point model and is not sensitive to the change in growth rate. Therefore, the first experiment shows that change in growth rate produces a change in the appearance of the behaviour, but they do not change the behaviour of the HLC. In the second experiment, the same approach was used to analyse the sensitivity of the immigration rate. Figure 6.7 shows that immigration rate has no impact on the behaviour of the HLC. Figure 6.9 shows the sensitivity of home leaving probability $\left(P_{h}\right)$. It can be observed that as the value of $P_{h}$ decreases beyond a certain value the household size at 40 years and 60 years tends to converge with the maximum household size. Therefore, the analysis shows that change in $P_{h}$ produces a change in the behaviour of the HLC and is a key parameter that may transform the HLC from an ideal point to a vector model.

In the final set of experiments, we analyse the sensitivity of couple formation probability $\left(P_{c}\right)$ on the behaviour of HLC. It can be observed from figure 6.10 that the household size at 40 years and 60 years tends to converge with the maximum household size as the value of $P_{c}$ increases. Therefore, similar to home leaving probability, couple formation probability is an important parameter that may transform the behaviour of the HLC from an ideal point to a vector model.

\subsubsection{Sensitivity of Time Step on Model Output}

The time step in the model is selected as 365 days ( 1 year) for three key reasons. First, most demographic events like birth, couple formation, couple dissolution and home leaving do not occur at a lesser resolution. For example, it is practical to assume that couples do not get separated every month. Similarly birth of a child to a mother can occur once a year. The output of the model is measured in terms of patterns of household life cycle which captures the change in family size annually. Understanding the output every month or at a higher resolution would not add any value with respect to the model objective. Therefore, to understand the impact of a 


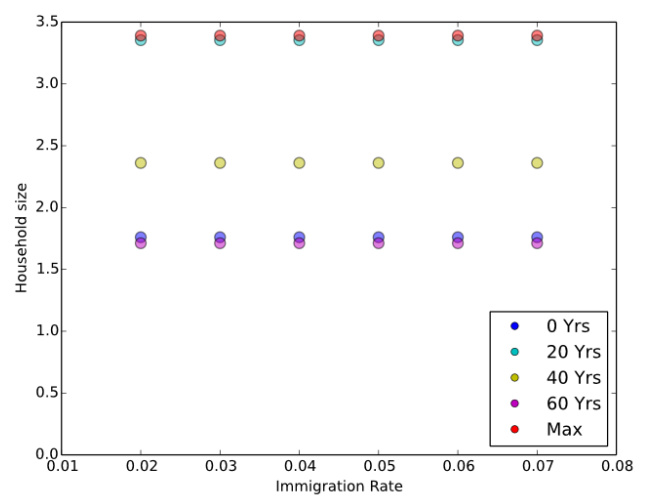

Figure 6.7: Sensitivity of Immigration Rate w.r.t. HLC.

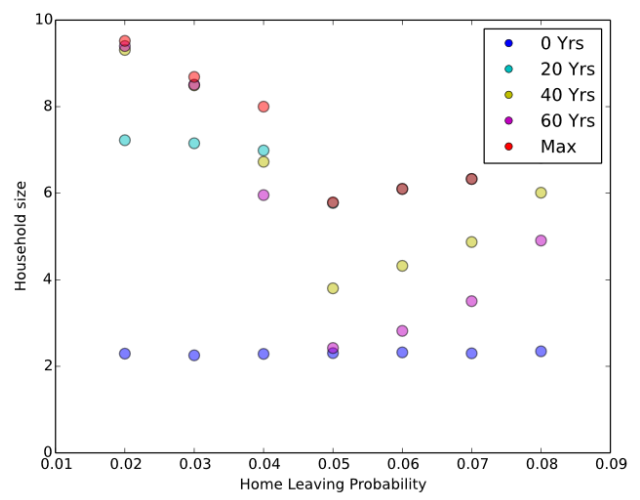

Figure 6.9: Sensitivity of Home Leaving w.r.t. HLC.

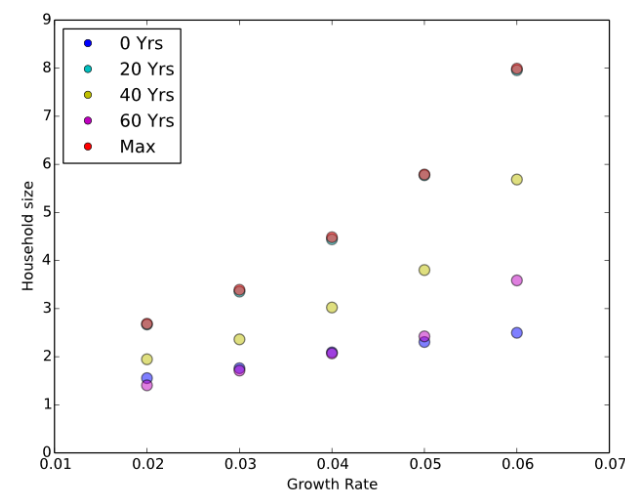

Figure 6.8: Sensitivity of Growth Rate w.r.t. HLC.

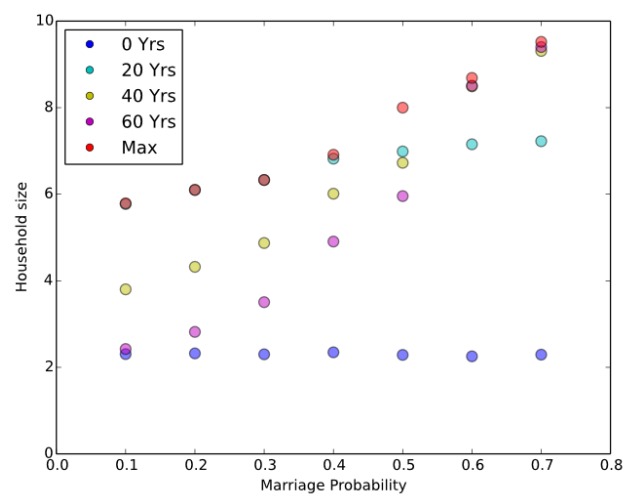

Figure 6.10: Sensitivity of Marriage Rate w.r.t. HLC.

Figure 6.11: Sensitivity Analysis of Dynaslum parameters with respect to HLC.

higher temporal resolution to the model output have run the model using $t=\{100,200,300,365\}$ as shown in figure 6.15 , which shows that the output of the model is consistent over different time steps.

\subsection{Results}

In this section, we present and discuss key results of the Population Dynamics Model and investigate the impact of home leaving and marriage on the HLC of slum and non-slum households. 


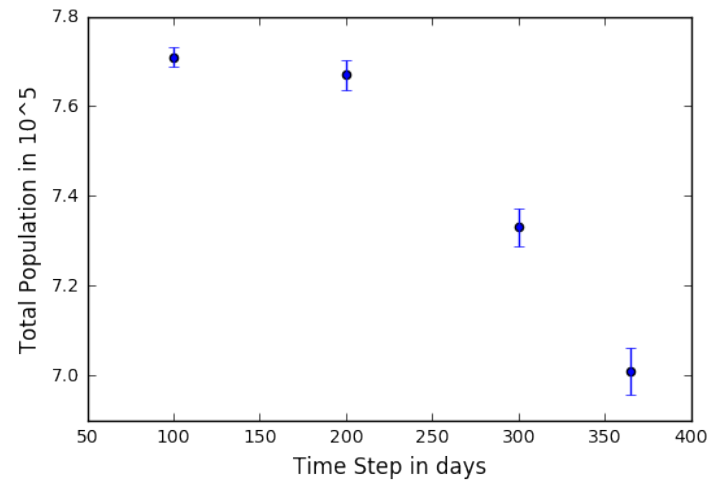

Figure 6.12: Total population over 30 runs.

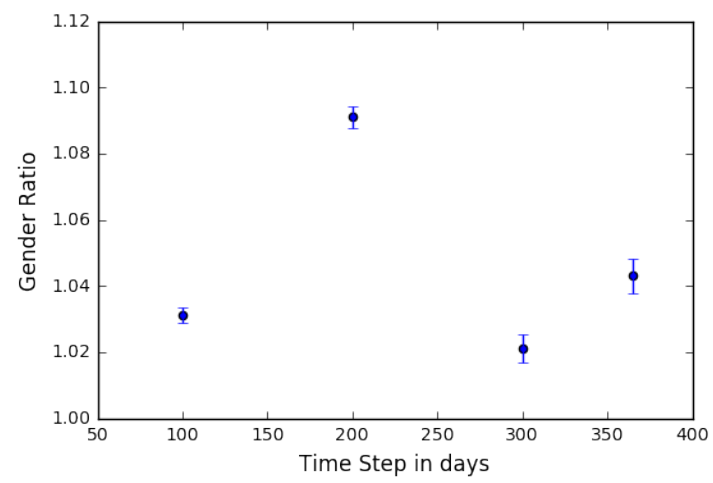

Figure 6.13: Gender ratio over 30 runs.

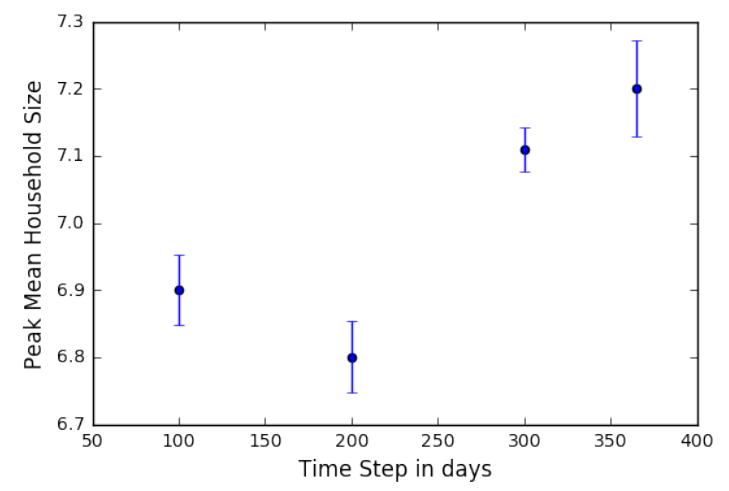

Figure 6.14: Peak mean Household size over 30 runs.

Figure 6.15: Mean and Standard deviation of Total Population and Gender ratio with different time steps.

\subsubsection{Difference in HLC between a slum and non-slum household}

Figure 6.5 and 6.6 shows a comparison between the simulated HLC of slum households with respect to the size of the household (figure 6.5) and the actual data from slums in Karnataka for the year 2005 (figure 6.6).

A household usually start its life-cycle with a single or a couple of individuals (e.g., a newly married couple). Thereafter, the size of the household increases steadily, peaking around twenty years (when, for example couples have children). Towards the end of the cycle, household size declines, reaching an average of 2 at around 50 years due to home leaving of children and death of parents. The period of maximum household size signifies the phase when most of the births occur in the household, but none or few of them have initiated their own family. 


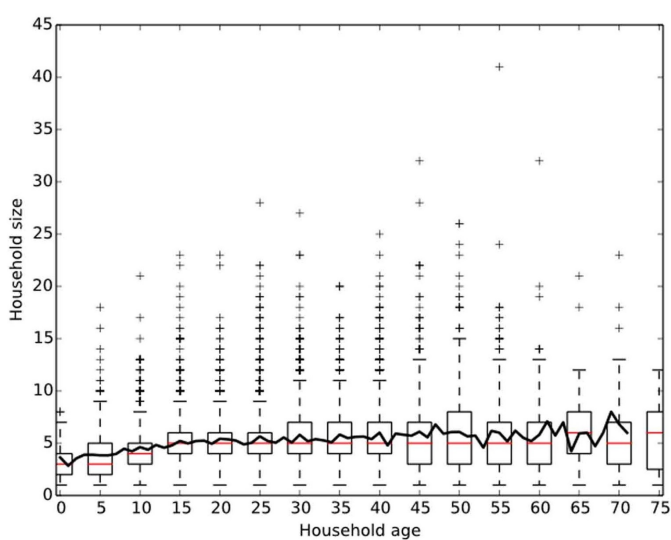

Figure 6.16: HLC in non-slum households of Karnataka in the year 1998 [3].

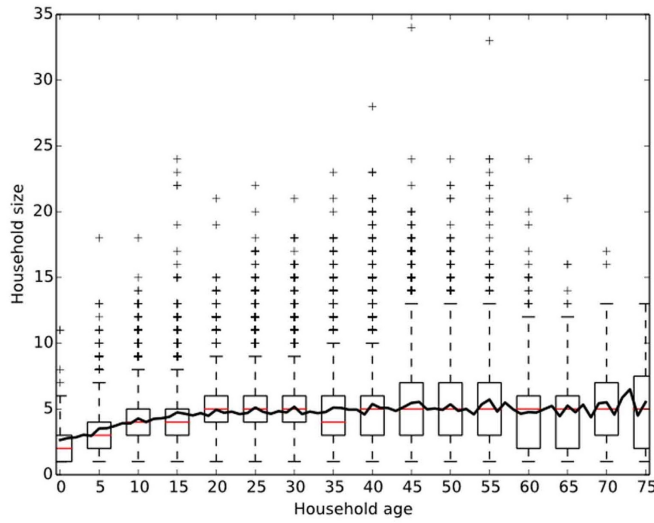

Figure 6.17: HLC in non-slum households of Karnataka in the year 2005 [2].

Comparing figure 6.6 and 6.17 we can see a significant difference between the HLC of slum and non-slum households. The HLC is crucial for urban planners; when building capacity for slum households (resettlement colonies) the planner must accommodate for the maximum household size. Large variation in household size during its life-cycle leads to inefficiency. Typically a family will continue to live in a larger house despite members having left. This leads to unused capacity and in turn puts pressure on the housing infrastructure. This emphasizes why understanding the HLC of slums is crucial for developing low cost housing for the slum dwellers. The non-slum HLC (figure 6.16 and 6.17 ) has a very stable household size throughout its life (between 3 and 4). However, the slum HLC varies significantly, growing from 2 early in the life-cycle, increasing to 6-7, and then decreasing back to 2-3 at the later stages in the life of the household.

Thus, it can be observed that a slum household tends to follow the ideal point model while a non-slum household follows a vector model. Here, an ideal point model, assumes that beyond a certain household age (threshold), household size decreases with increase in household age. A vector model, by contrast, can be defined as one where the household maintains a stable household size beyond a certain household age (threshold). Through sensitivity analysis with Dynaslum we find that home leaving and couple formation are the two key parameters that may transform the HLC from an ideal point to a vector model. 


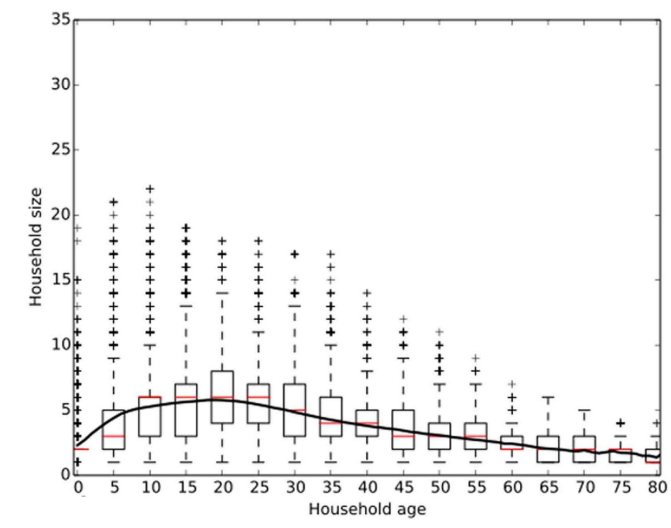

Figure 6.18: Simulated Household Life Cycle: $\delta_{g}=0.05, P_{h}=0.05$.

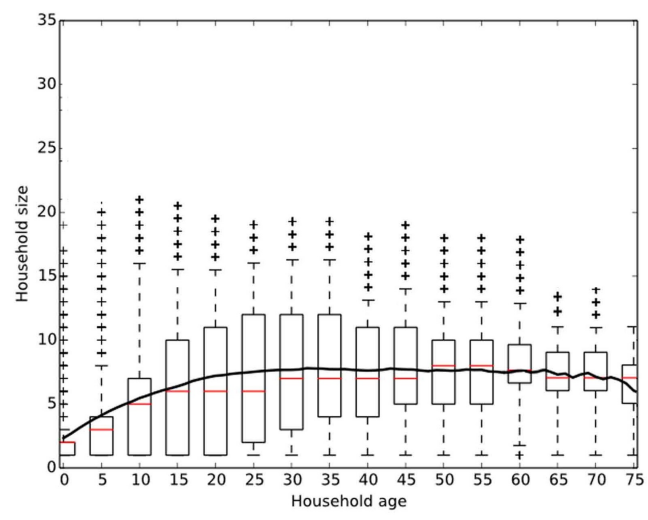

Figure 6.19: Simulated Household Life Cycle: $\delta_{g}=0.05, P_{h}=0.025$.

\subsubsection{Impact of Home-Leaving on HLC}

By means of Dynaslum we explore the impact of home leaving (forming new household) among young people in informal settlements (slums) in a simulated environment to establish the differences in HLC between slum and non-slum households in a city. Home leaving is an important event because of its inter-dependencies with other markers of the transition to adulthood and association with risk behaviour [161]. By varying the home leaving probability $\left(P_{h}\right)$, while keeping other parameters fixed as described in table 6.1 we study the impact of home leaving on HLC. It can be observed from figure 6.18 and 6.19 , that for a given growth rate $\left(\delta_{g}\right)$, a ratio of growth rate and home leaving probability $\left(\delta_{g} / P_{h}\right)$ closer to the value of 1 indicates an ideal point model. The HLC tends to approach a vector model as the home leaving probability $\left(P_{h}\right)$ is decreased. Figure 6.19 indicates that at $\delta_{g}=0.05, P_{h}=0.025$, the HLC is similar to patterns observed in non-slum households (see figure 6.17). This experiment highlights one way by which to transform a slum HLC to the general urban HLC. This may imply that the key difference between the slum population and the general urban population is that young adults in slums are more likely to leave the parental home once they reach the age of 18 . This in turn implies that the average age at which young slum dwellers leave the parental home is lower than for non-slum households. Most of the earlier studies relates home leaving to a personal choice or an independent decision of the young individual concerned. However, there is evidence that floor space can influence leaving the parental home. For example, it has been found that in slums, a higher number of siblings increases the likelihood of leaving home for couple formation 


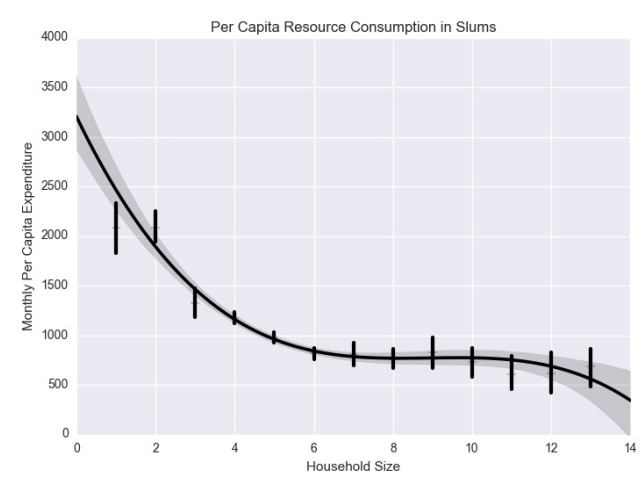

Figure 6.20: Resource consumption in slums of Bangalore from survey data.

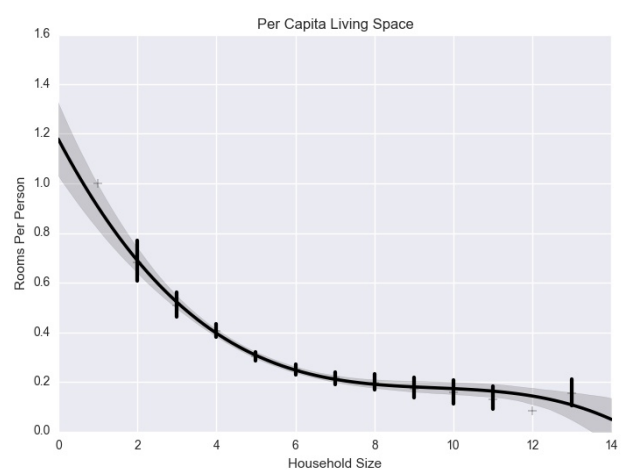

Figure 6.21: Living space per capita in slums of Bangalore from survey data.

and employment reasons; however, it decreases the likelihood of leaving home for furthering education $[162,163]$. Therefore, home leaving may be reduced by building houses with higher floor space per capita (or higher floor space index) which can in turn reduce the formation of new slum households.

Further, the experiment also indicates that reduction in average household size due to higher home leaving alone will increase the number of single-person households by $21 \%$. In other words, had the average household size remained at the maximum level of around 6 (figure 6.19), there would have been $21 \%$ fewer slum households. The decrease in slum household size due to home leaving along with increasing population growth rate creates an enormous stress on the housing infrastructure. First, more households mean more housing units, thus normally increasing the amount of land and resources needed for housing construction. Second, smaller person households have lower efficiency of resource use per capita while in larger households, goods and services are shared by more people $[164,165]$. We use a unique and novel dataset based on the field work in the slums of Bangalore, to understand the effect of household size on optimal resource consumption. We perform a regression analysis of the per capita expenditure and living space with respect to the household size. Figure 6.20 and 6.21 shows that the per capita resource consumption and living space decreases with an increase in household size until an optimal household size of 6. A comparison of figure 6.18 and 5.5 indicates that slum households try attain this optimal household size of 6 but fail to maintain this optimal state.

Therefore, the above findings show that reducing home leaving in slum households would lead to a stable household size of around 6 (as seen in figure 6.19), which in turn decreases the rate of formation of new slum households and enable optimal per capita resource consumption. 


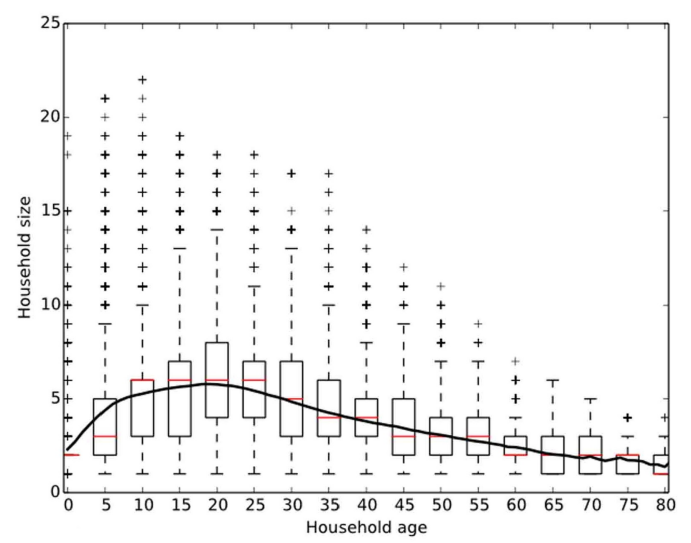

Figure 6.22: Simulated Household Life Cycle: $\delta_{g}=0.05, P_{c}=0.1$.

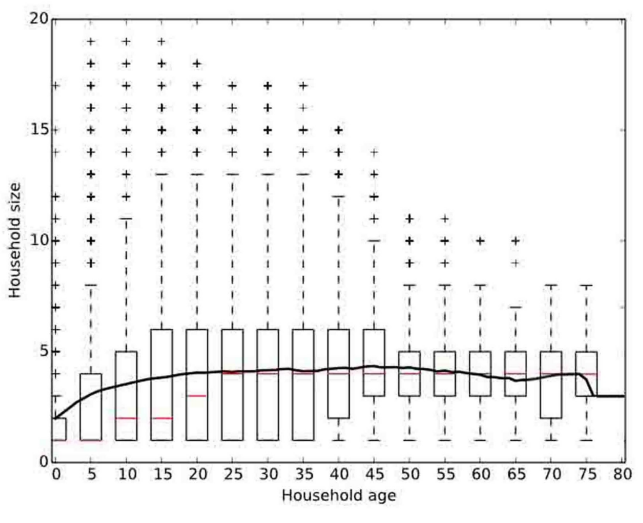

Figure 6.23: Simulated Household Life Cycle: $\delta_{g}=0.03, P_{c}=0.6$.

\subsubsection{Impact of Couple Formation on HLC}

In this section we discuss the impact of couple formation among adults on the HLC. By varying the growth rate $\left(\delta_{g}\right)$ and the annual couple formation probability $\left(P_{c}\right)$, whilst keeping other parameters fixed as described in table 6.1 we study the impact of couple formation on HLC. Figure 6.22 demonstrated the life cycle of a slum household where most marriages lead to formation of new households. However, figure 6.23 shows that as $P_{c}$ is increased from 0.1 to 0.6, the HLC tends to approach a vector model similar to a non-slum household.

In Dynaslum, as far as HLC is concerned, higher couple formation probability is synonymous with more couples merging with their parent household as any new household formed does not influence the behaviour of the life-cycle. Therefore, it can be observed from figure 6.22 and 6.23 that young couples in slums are more likely to be living independently as compared to couples in non-slum households. The association of independent living with marriage reinforces previous studies showing that young couples may leave home in anticipation of greater freedom and privacy, given the crowded living space in slum dwellings [161]. However, in case of a non-slum household, married couples may not form an independent household, but remain part of an existing family household [166].

Similar to home leaving, the experiments indicate that an increase in the number of new households after marriage leads to a $15 \%$ increase in the total number of slum households, which may manifest into the growing urban slum sprawl. 


\subsection{Discussion}

The population size of slum dwellers and growth rate are often considered important drivers of biodiversity loss in cities of developing countries, whereas the impact of household dynamics is usually excluded from the analysis. Measuring aggregate demographic indicators may mask significant changes in the household size and the number of households, and their impact on the ecosystem. Household life cycle influence per capita consumption, for example, consumption of fuel, water, electricity, habitat alteration for home building and associated activities, and greenhouse gas emissions [20]. In this chapter we have developed an agent based model, namely Dynaslum, to study the impact of socio-demographic processes on the life cycle of slum households. We find that a higher rate of independent living among the youth leads to formation of higher number of smaller slum households.

There are very few studies that have experimentally investigated home leaving of youth in an urban slum setting, perhaps because it is construed as difficult to disentangle the impacts of home leaving from the multitude of additional factors that influence HLC. We have demonstrated, however, that with a computational approach the impacts of home leaving and marriage can be readily investigated. An increase in smaller slum household numbers and resultant higher per capita resource consumption in smaller households challenges current urban planning practices in fast-growing cities of developing countries. Therefore, by building resettlement colonies that can accommodate a larger family (higher floor space per capita) and provide positive youth development opportunities, home leaving of young adults can be reduced. This in turn will reduce the number of slum households substantially, and will allow the policy makers to plan efficient resettlement colonies for a stable slum household size. 
6. POPULATION DYNAMICS MODEL 


\section{Chapter 7}

\section{Residential Choice Model}

In this chapter we describe a multi-agent discrete-choice model of residential choice for the slum dwellers in the city of Bangalore, India, using census of India and the survey data (described in chapter 4). Individual preferences and neighbourhood characteristics, along with social capital, will be used to capture micro behaviour regarding residential preferences. In addition, this study implements the theory of planned behaviour based on economic, social and environmental factors in non-binary choice models. Discrete-choice models have been used in many applications, such as, transportation systems [167, 168, 169], wildlife resource selection [170], healthcare [171] etc. However, the application of discrete-choice models to understand the residential choices of slum dwellers is new and realistic as compared to iterative-approach (described in chapter 3) employed in previous slum models. Incorporating the residential choice dynamics in Dynaslum, will enable the matching of households (generated in chapter 6) to their residential choices based on economic, social, and environmental satisfaction. It may be expected that a slum located in an inexpensive environment but farther away from job sites will be more appealing to slum households comprising of elderlies, whereas young people pursuing a job would prefer a slum which provides a balance to their social, environmental and economic well-being. Affordable housing and jobs deliver economic satisfaction, proximity to friends and family or social vitality determines social satisfaction, and the quality of neighbourhood provides environmental satisfaction. The challenges for policy makers to incorporate well-being and quality of life into their considerations are diverse. First, as discussed above, assessment of human well-being includes several dimensions and varies between individuals and different sections of society. Second, as each individual has a social network within which people share their belief and information, they exercise normative influences on each other. For example, 


\section{RESIDENTIAL CHOICE MODEL}

family and friendship are important determinants to residential decisions. Third, interdependencies between people and households may have a geographical component. Though people often prefer to work in close proximity to their social environment, it is usual that individuals reside and work in different slums or municipalities. In the case of poly centric cities in developing countries, one slum may provide better economic opportunities, whereas another slum could offer better social and environmental quality (or cheaper housing), and commuting becomes a strategy to optimize social, economic and environmental satisfaction [172]. However, if the commuting distance becomes large this may have a negative impact on the quality of life, indicating that policy should not focus on single slum, but rather study the city as a network of slums each offering different qualities.

The Residential Choice Model in Dynaslum aims at understanding and explaining the underlying factors that influences the residential choices of slum dwellers in Bangalore, based on empirical evidence shown in chapter 4. In particular, the objective of this chapter is to answer the following questions:

1. What are the key underlying factors that influences residential mobility?

2. How does the existing social, economic and environmental situation impacts the choices of slums?

3. How do social networks facilitate and influence residential location choices in slums of Bangalore?

While few studies have been presented to understand the possible factors influencing slum dwellers to choose a particular slum, modelling this phenomenon is a challenge as it is a complex process, guided by heterogeneity in choices, dynamic reactions to constraints and opportunities embedded in the urban setting within which the decisions are made. The structure of this chapter is as follows. First, in section 7.1 we review the literature to understand existing concepts and theories of residential choice behaviour. Second, in section 7.2 we describe the design and implementation of the agent-based residential choice model. The ABM described in this section will include social and neighbourhood quality in addition to economic motives as the key drivers to residential choices. The agents in the model are connected using social networks and can share information and beliefs with each other. The empirically parametrized model would be able to make fine-grained projections of residential choices of slum dwellers in the city of Bangalore. Third, in section 7.3 we present the validation and the sensitivity 
analysis of the residential choice model. In section 7.4 we discuss the results of the model which relates to the objective stated in this section. Finally, in section 7.5 we conclude this chapter with a discussion and policy implications of the findings.

\subsection{Related Work}

In this section, first, we discuss the two existing important approaches used to model the residential choice behaviour, namely: the choice approach and the bid-rent approach. Second, we review the existing microsimulation models designed to study spatial mobility of households.

\subsubsection{The Choice and Bid-Auction Approach}

In the last three decades, Discrete choice models (DCM) and random utility models (RUM) in particular, have been widely implemented in numerous applications. RUM provides a microeconomic framework to model residential choice of a household by maximizing the combined utility function given existing income constraints. Households select a list of services $x$ and specific location, $i$, characterized by a set of physical attributes of the location $\left(z_{i}\right)$ as shown in equation 7.1 [173].

$$
\max _{x, i} U\left(x, z_{i}\right)
$$

The combined utility of the household is constrained by income $I$ as shown in equation 7.2:

$$
p * x+r_{i}<I
$$

where $p$ is total expenditure on goods and $r_{i}$ is the rent for the location. The household's utility at a given location, $i$, comprises of the conditional indirect utility $V$ (optimal solution to the utility maximization) and an error component which explains the heterogeneity in households decisions. Therefore, the probability that a household will relocate from location $j$ to $i$ will depend on the perceived gain in utility as shown in equation 7.3:

$$
P(i \mid h)=P\left(V_{i h}+\epsilon_{i}>V_{j h}+\epsilon_{j}, \forall i \neq j\right)
$$




\section{RESIDENTIAL CHOICE MODEL}

where $h$ denotes the household and $\epsilon$ is the random error term. Assuming an extreme value distribution for $\epsilon$, the probability of an household $h$, choosing a location $i$ from a set $s$ is given as:

$$
P(i \mid h, s)=e_{i h}^{V \mu} / \Sigma j \epsilon s^{e_{j h}^{V \mu}}
$$

where $\mu$ is a positive scale parameter. The expression in equation 7.4 is also known as Multinomial Logit (MNL) which evolved to different choice models such as nested logit or probit. However, according to McFadden (1978) MNL will lead to biased results if the alternatives in choice set are correlated [174]. Therefore, in order to capture the correlations a nested logit (NL) model was introduced where the probability of a household $h$ choosing a location $i$ is defined as:

$$
P(i)=P(i \mid m) \cdot P(m)
$$

where $m$ is the nest ${ }^{1}$ and $i$ is the location alternative, and $P(m)$ is the marginal probability of choosing nest $m$ while $P(i \mid m)$ is the conditional probability of $i$ being chosen, given the nest $m$. The NL models improved the MNL models as they captured the correlations by nests. However, it was criticised as it failed to capture heterogeneity, i.e. different sensitivities by households to same attributes. To overcome this problem, Walker (2001) introduced Hybrid Choice model (HCM) to include latent variables such as perceptions to account for the heterogeneity [175]. Walker and Li (2007) apply the HCM approach to incorporates quality of life as a latent class to model residential choice [176]. The above choice based approach focuses on individual decision making. However, the other approach which has been widely used to model residential choice is the bid-auction which captures the interactions among households in the real estate market. The assumption is that households bid for dwelling based on their willingness to pay (WTP) in an auction based market. The household with the highest bid or rent $\left(r_{i}\right)$ will be offered the dwelling at the bid amount as shown in equation 7.6.

$$
r_{i}=\mathbf{E}\left(\max _{h \in H}\left(V_{i h}\right)\right)
$$

Gross (1990) demonstrates that the bid-auction model works well in estimating housing price when households are considered as a homogeneous group. Chattopadhyay (1998) concludes that

\footnotetext{
${ }^{1}$ Nests are groups of available choices.
} 
the bid-auction approach is better as compared to hedonic approach using different groups of agents. Muto (2006) finds systematic difference between land value and land use type. The bid function does not include the price of the location and hence does not have the problem of price endogeneity. Endogeneity occurs when prices are highly correlated with unobserved attributes of the location, leading to erroneous estimates.

\subsubsection{Estimating Bid Function}

In this section we estimate the bid functions for slum choice using a latent variable approach demonstrated by Hurtubia and Bierlaire [177], namely the latent auction model. The model is estimated for the data collected from 37 slums in Bangalore, described in chapter 4 . We consider several household attributes and spatial attributes that are relevant to the choice of slums by individual households. Table 7.1 shows the household characteristics that are considered as attributes of the decision maker. The slums are described by traditional socioeconomic variables like average income in each slum, percentage of Hindus or Muslims in a slum, presence of economic activities by each type and average distance to health centres. The household attributes are interacted with the spatial attributes to estimate the heterogeneity in the choice of slums as shown in table 7.1. The bid function $B_{h i}$ represents the willingness to pay of household $h$ to settle in slum $i$. As shown in table 7.1, the average surface area in a slum is interacted with the logarithm of the number of individuals in the household to account for bigger (but diminishing) demand for space of large households. The percentage of households with Muslim inhabitants in a slum is interacted with a dummy variable that indicates if a household is Hindu. Similarly, the percentage of households with Hindu population in a slum is interacted with a dummy variable that indicates if a household is Muslim ${ }^{1}$. Segregation by income was also analysed by interacting proportion of low income households in a slum with a dummy variable indicating if a household is high income. The percentage of people in a ward with a university degree is interacted with a dummy variable indicating that at least one member of the household holds a degree. Accessibility to healthcare and education was interacted with a dummy variable indicating if the household has children or not.

Table 7.2 shows the results of the estimation of the Latent Auction model. The coefficients indicate that the (willingness to pay) WTP for surface increases with household size. We see that Muslim households have stronger preference to reside in a slum with Muslim majority

\footnotetext{
${ }^{1}$ Hindus and Muslim are the two largest religion (89\% of population) in the slums of Bangalore. Chapter 4 shows that spatial segregation based on religion is highest between Hindus and Muslim.
} 


\begin{tabular}{|c|c|c|c|}
\hline Parameter & Spatial Attribute & $\mathrm{x}$ & Household Attribute \\
\hline$A S C_{2}$ & - & & Religion Constant (Hindu) \\
\hline$A S C_{3}$ & - & & Religion Constant (Muslim) \\
\hline$A S C_{4}$ & - & & Religion Constant (Christian) \\
\hline$\beta_{e d u c}$ & $\begin{array}{l}\% \text { of blue collar jobs in } \\
\text { slum } i\end{array}$ & $\mathrm{x}$ & dummy for hh's with children \\
\hline$\beta_{\text {self }}$ & $\begin{array}{l}\% \text { of self employed jobs in } \\
\text { slum } i\end{array}$ & $\mathrm{x}$ & $\begin{array}{l}\text { dummy for hh's with Religion }= \\
\text { Muslim }\end{array}$ \\
\hline$\beta_{i n c_{h}}$ & $\begin{array}{l}\% \text { of income level } 1,2,3 \text { in } \\
\text { slum } i\end{array}$ & $\mathrm{x}$ & $\begin{array}{l}\text { dummy for hh's with income } \\
\text { level } 7,8,9\end{array}$ \\
\hline$\beta_{i n c_{l}}$ & $\begin{array}{l}\% \text { of income level } 7,8,9 \text { in } \\
\text { slum } i\end{array}$ & $\mathrm{x}$ & $\begin{array}{l}\text { dummy for hh's with income } \\
\text { level } 1,2,3\end{array}$ \\
\hline$\beta_{\text {surf }_{h}}$ & surface of dwelling $v$ & $\mathrm{x}$ & $\begin{array}{l}\text { dummy for hh's with income } \\
\text { level } 7,8,9 \text { and size }>2\end{array}$ \\
\hline$\beta_{\text {surf } f_{l}}$ & surface of dwelling $v$ & $\mathrm{x}$ & $\begin{array}{l}\text { dummy for hh's with income } \\
\text { level } 1,2,3 \text { and size }>2^{1}\end{array}$ \\
\hline$\beta_{a c c_{w}}$ & $\begin{array}{l}\text { average distance to water } \\
\text { source in slum } i\end{array}$ & $\mathrm{x}$ & $\begin{array}{l}\text { dummy for hh's with income } \\
\text { level } 1,2,3\end{array}$ \\
\hline$\beta_{a c c_{h}}$ & $\begin{array}{l}\text { average distance to health } \\
\text { centre in slum } i\end{array}$ & $\mathrm{x}$ & $\begin{array}{l}\text { dummy for hh's with income } \\
\text { level } 7,8,9\end{array}$ \\
\hline$\beta_{r e l_{h}}$ & $\%$ of Muslim in slum $i$ & $\mathrm{x}$ & $\begin{array}{l}\text { dummy for hh's with Religion = } \\
\text { Hindu }\end{array}$ \\
\hline$\beta_{r e l_{m}}$ & $\%$ of Hindu in slum $i$ & $\mathrm{x}$ & $\begin{array}{l}\text { dummy for hh's with Religion = } \\
\text { Muslim }\end{array}$ \\
\hline
\end{tabular}

Table 7.1: Interaction of Spatial Attribute and Household Attribute for Latent Auction Model.

population. Similar preference exists among Hindu households but is significantly weaker. The results also indicate that segregation based on education and healthcare, accessibility to health care and education is statistically non-significant. The results indicate high degree of heterogeneity in the choice of slums based on the current situation of individual households. It also indicates that social and economic factors dominate the decision making process. However, in case of the slums the choice sets, attributes and individual behaviour are dynamic and cannot be designed a priori. Therefore, we find that the bid function approach is not sufficient to capture emergent phenomenon and critical transitions in the residential choice process of slum dwellers as there are various heterogeneous social groups formed by religion, language and income, but the approach is used to derive behavioural rules and guide the parametrization of the agent-based residential choice model in section 7.2.2. 


\begin{tabular}{|l|l|l|l|}
\hline Parameter & Value & Std. Error & t- test \\
\hline$A S C_{2}$ & $-0-0496$ & 0.21 & -0.24 \\
$A S C_{3}$ & -0.442 & 0.223 & -1.97 \\
$A S C_{4}$ & -3.4 & 0.181 & -4.15 \\
$\beta_{\text {educ }}$ & -0.96 & 0.12 & 2.25 \\
$\beta_{\text {self }}$ & 0.269 & 0.528 & -4.13 \\
$\beta_{\text {inc }_{h}}$ & 0.562 & 0.08 & 3.09 \\
$\beta_{\text {inc }_{l}}$ & 0.755 & 0.079 & -1.12 \\
$\beta_{\text {surf }_{h}}$ & 0.935 & 0.032 & 4.49 \\
$\beta_{\text {surf }_{l}}$ & 1.12 & 1.08 & 6.76 \\
$\beta_{\text {acc }}$ & -0.327 & 2.07 & 4.32 \\
$\beta_{\text {acch }_{h}}$ & -1.2 & 1.2 & 3.09 \\
$\beta_{\text {rel }_{h}}$ & -3.07 & 0.03 & 2.09 \\
$\beta_{\text {rel }_{m}}$ & -0.003 & 0.532 & 1.95 \\
\hline
\end{tabular}

Table 7.2: Estimation of DCM coefficients for slums in Bangalore.

\subsubsection{Microsimulation models}

Micro-simulation modelling was first introduced by Orcutt (1957) [178]. Ben-Akiva and Bowman (1998) present a theoretical framework for microscopic modelling of residential, activity, and travel decisions for households and individuals, and the interactions between urban development, household decisions and transport system performance [179]. Researchers have long tried to estimate households' spatial movement between dwellings within an urban area. Existing residential mobility models tend to focus on modelling the process of households' moving decisions and choices. Researchers have built several residential mobility microsimulation models and applied them to the context of different countries and regions all over the world. While few models focus on choice between alternative dwellings, other models attempt to capture changes in demand for new dwellings and the effect of migration.

Previous reviews of existing microsimulation models highlight their limited scope or capabilities with respect to slums. IRPUD and RURBAN are capable of only zonal level modelling, SEVIRAGE forecast at regional level while SMILE projects the population at a national level. The other residential mobility models, although simulating behaviour at the individual level, tend to do a poor job of considering the interaction among the individuals or households in the housing market and fail to explicitly incorporate the housing market mechanism, which may limit the results of the models. In order to capture the interactions among individuals and households and to represent the housing market where different agents (individual/households, 


\section{RESIDENTIAL CHOICE MODEL}

firms, and real estate developers) interact with each other, most of the existing models adopts an equilibrium approach, wherein the supply equals demand and market clearing is achieved by imposing the equilibrium condition and finding bids that produces it. However, these models should be adapted significantly to study slums, as the notion of formal supply is non-existent and informal in case of slums.

Table 7.3 compares key features among existing microsimulation models and shows that we need a new non-equilibrium model that incorporates social, economic and environmental factors to simulate residential mobility. The design of the residential choice model is introduced in detail in the next section.

\subsection{The Model}

In this section we describe the Residential Choice Model following the ODD + D protocol [180], which extends the ODD protocol [155]. First we describe the overview and the decision making rationale in the process of changing an activity for an individual. Secondly, the key concepts involved in the process are explained which is based on satisfaction maximization driven decision making. Finally, the process of a household's decision to move from its current to a new residential location is described in this section. While we have drawn upon features from existing models $[175,172]$ in the development of the residential choice model, we observe that these models do not completely satisfy our objective of understanding social, economic and spatial mobility of slum dwellers.

\subsubsection{Overview}

\subsubsection{Purpose}

The residential choice model in Dynaslum provides a model for individual decision making with respect to spatial mobility based on maximization of a satisfaction function of social, economic, and environmental parameters for slum dwellers in Bangalore. It also provides a framework to formalize heterogeneous individual social behaviour with respect to economic mobility.

\subsubsection{Entities, state variables, and scales}

The model comprises of three levels of entities: individuals, households, and slums. An individual is characterized by the state variables as listed in table C.1, Appendix C. A household consists of a number of individuals who have a family relationship and live together. Table C.2, 


\begin{tabular}{|c|c|c|c|c|c|c|c|c|c|}
\hline & 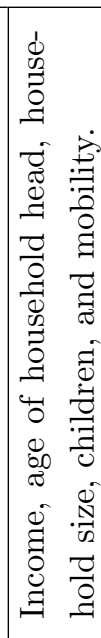 & 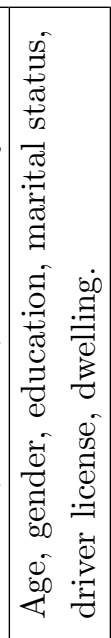 & 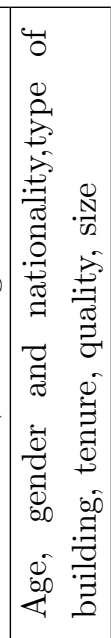 & 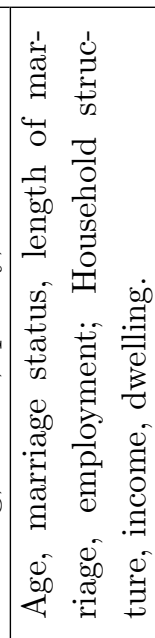 & 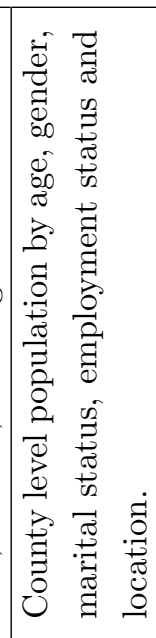 & 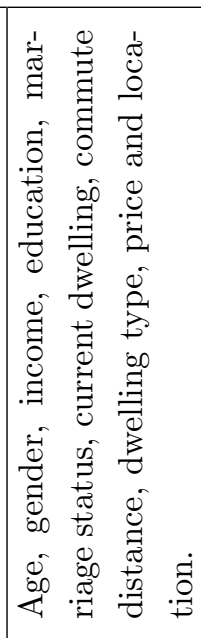 & 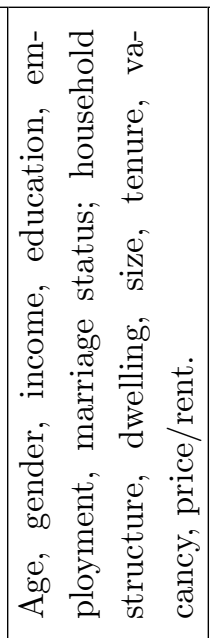 & 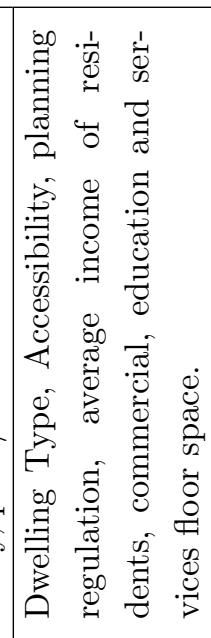 & 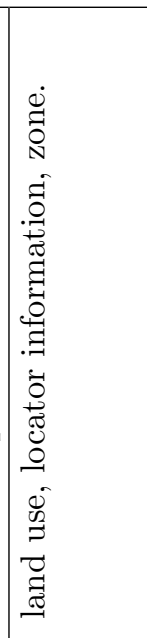 \\
\hline $\mid$ & 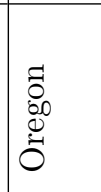 & $\begin{array}{l}0 \\
\stackrel{0}{0} \\
0 \\
0 \\
0 \\
\oplus\end{array}$ & 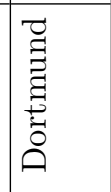 & 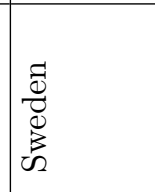 & 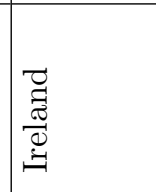 & 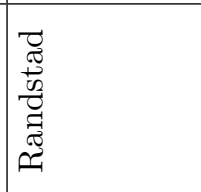 & 㩊 & 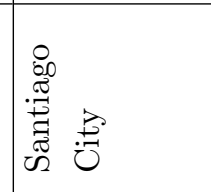 & 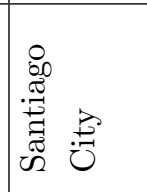 \\
\hline 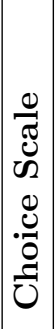 & . & 苛 & Dี๊ & 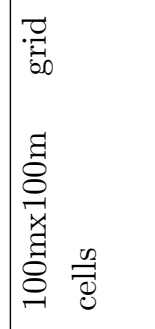 & 童 & 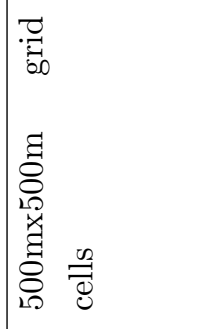 & 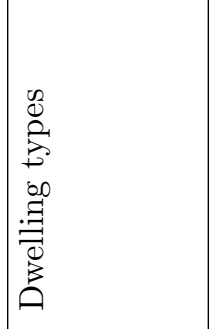 & 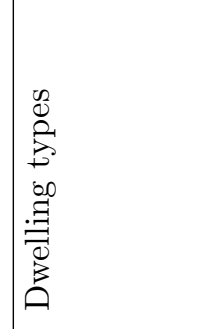 & 芯 \\
\hline 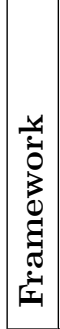 & 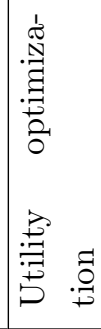 & 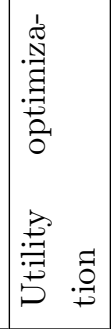 & 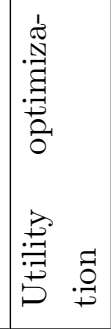 & 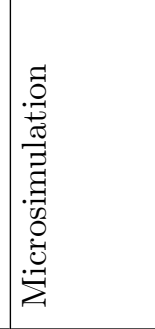 & 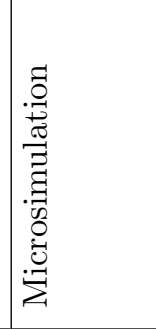 & 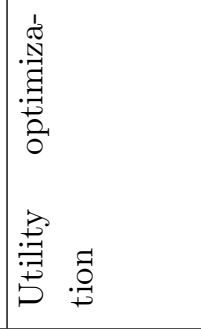 & 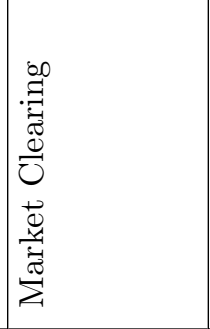 & 常 & 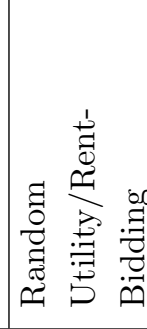 \\
\hline 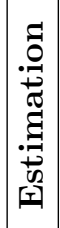 & 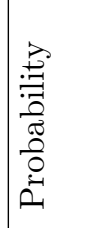 & 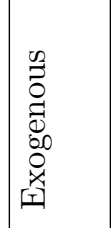 & 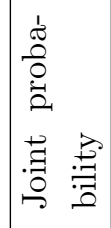 & 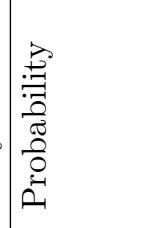 & 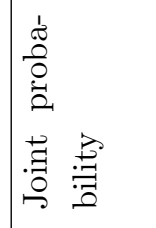 & 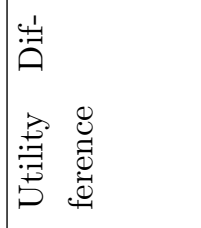 & 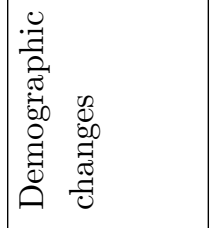 & 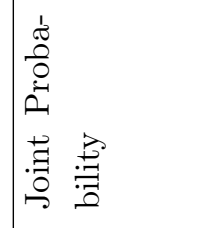 & 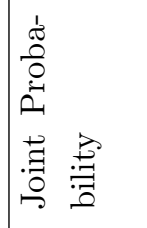 \\
\hline 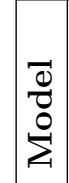 & 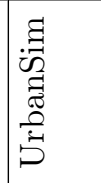 & 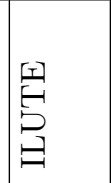 & 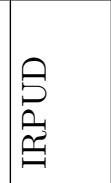 & 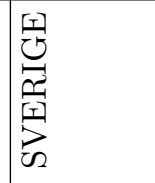 & 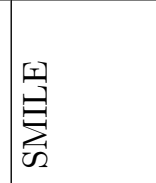 & 起 & 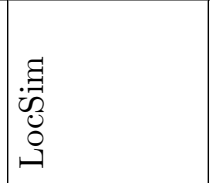 & $\begin{array}{l}n \\
w \\
0 \\
z\end{array}$ & 荒 \\
\hline
\end{tabular}

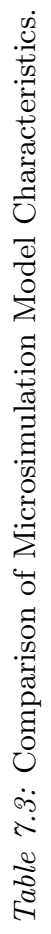




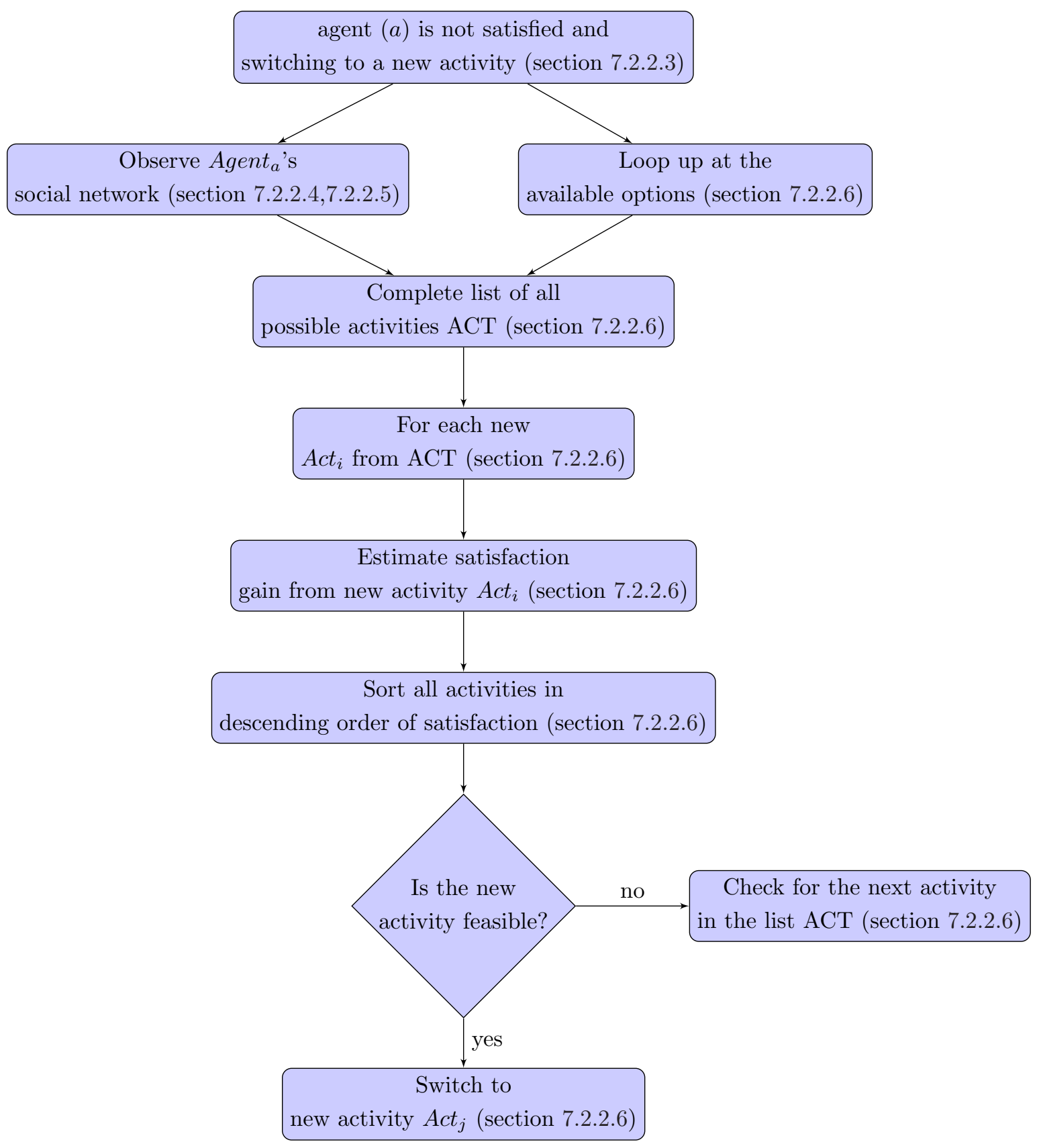

Figure 7.1: Decision making process of an individual agent (see section 7.2.2.3) Each block contains the section where the process is explained in details. 
Appendix C lists the state variables of a household. A slum is the environment that a household and individuals live or work in. There are three types of slums in terms of their locations: core, intermediate and periphery. The state variables of a slum are listed in table C.4, Appendix C.

\subsubsection{Process overview and scheduling}

The process describing an agent's behaviour with respect to changing activity is primarily based on maximization of collective satisfaction derived from agent's social, economic, and environmental needs. Suppose an agent $(a)$ is undergoing an activity ${ }^{1} A_{i}$ at time $T$, if the agent $(a)$ is not completely satisfied with the collective outcome of such an activity, the agent (a) will look to change its activity at time $T+1$ to a new activity $A_{j}$. Now, lower the collective satisfaction derived from its current activity, higher the chances that agent $(a)$ will switch to a new activity at next time step. Therefore, the initial task of agent $(a)$ is to update information regarding available activities it can take at the next time step. This can be achieved by asking other individuals (agents) in it's social network or by exploring new activities from a list of vacancies. Once, the information is collected, the next step is to calculate the expected collective satisfaction from the new activity $A_{j}$. The higher the collective satisfaction, the more likely that agent $(a)$ will switch to the new activity $A_{j}$. The following flowchart presented in figure 7.1 summarizes the various steps involved in the process of changing an activity for an agent.

The second process maintains the list of available occupations in each slum as shown in the table C.3, Appendix C. In each slums, the jobs are characterized by a job id, salary, number of vacancies and expiry date. At each time step, new occupations are stochastically created based on empirically observed distribution of jobs (described in 4, section 4.2.3, figure 4.11) in the slums and the expired jobs are removed. The purpose of the above process is to incorporate economic mobility (upward and downward) in the model. Downward mobility occurs when an agent moves to a lower income job or the job expires. Upward mobility is a situation when an agent is able to find a higher income job.

\subsubsection{Design Concepts}

\subsubsection{Theoretical Background}

In context of cities, residential choice, can be defined as a matching procedure, during which social agents like households choose among residential alternatives. It leads to spatial mobility triggered by the change in an agent's social, economic and environmental well-being. In this

\footnotetext{
${ }^{1}$ An activity is defined as current state of residence and employment
} 


\section{RESIDENTIAL CHOICE MODEL}

section, we review the literature to provide a theoretical framework to formulate the residential choice model.

\subsubsection{Concepts And Theories}

Residential choice concepts and theories help to explain the spatial mobility of households. In the context of land use and residential mobility, previous studies mainly focus on the relocation process and the trigger to the relocation. On one hand, urban economists try to understand the patterns in land use as a function of household location choices and income, which further impacts the price of the real estate. On the other hand, sociologists study the emerging social structure and segregation based on residential choices. In this section we will provide a brief review of existing theories developed by researchers from different disciplines (sociologist, economist, planners).

- Accessibility to workplace: In 1960s, Alonso proposed a spatial model of residential choice for a developed country scenario [181] which demonstrates the concept of residential choice based on concentric regions around the Central Business District (CBD) where the economic activities are situated. The residential choice is influenced by the price of housing, space and cost of commuting. The model assumed that all the households optimized costs between rent and travel expenses. Similar theory for residential choice were explored based on alternative contexts such as multi-centred cities, lifestyles, values and culture [182]. A more comprehensive theory on residential choice was provided by Richardson (1977) as compared to Alonso's trade-off model [183]. He explained that the trade-off model was a simplified version and failed to explain the availability and constraints of the houses in a poly-centric environment. Phe and Wakely also indicated that Alonso's model of household choices is limited as it is sensitive towards the variations in workplace accessibility $[184,185]$. The recent urban area sizes including work and shopping cannot be concentrated in one place. The residential choice may not only depend on $\mathrm{CBD}$ as the urban centres are increasingly becoming crowded which leads to congestion. The trend of informal service centres have increased mainly in African cities enhancing the services found in the CBD. A perfect example is Dar es Salaam where the establishment of CBD services is rapidly increasing.

Phe and Wakely proposed a more rationalised theory [185] where a location is chosen based on one's status with a suitable quality of life. In case the household is underprivileged, it 
will try to stay close to households with similar socio-economic background, as opposed to a better quality dwelling. Therefore, a dwelling's desirability highly depends on one's perceived status attached to housing. Evidently, the earlier studies used an econometric approach and focused on the individual as a coherent decision maker with an objective to maximise utility.

- Neighbourhood, Environment and the Community: The role of neighbourhood is only partially clarified in the literature on residential mobility and housing choices. According to Clark et al, neighbourhood is an individual entity and highly influence the decision making [186]. The study was based on the Netherlands Housing Survey 1998 to understand household residential mobility with respect to different neighbourhoods based on socio economic and environmental status [186]. Social elements such as schools, healthcare, pollution, water and community spirit contribute towards the likeliness of a neighbourhood [187, 188]. In developing countries, social interaction and community is important and the presence of lower income household is acceptable, who provide basic services to the higher income households [185].

- Social Capital: Social capital is based on networks of mutual sustenance that exist between households, social groups, and communities who can provide information about employment and opportunities [189]. The sustainable livelihoods approach by Farrington et al. (2002) indicates urban India shows the significance of social network in finding work and accommodation [190].

- Household Life Cycle: Peter Ross's study in Philadelphia, suggested that residential mobility is a function of the household's adjustment to changes in household life cycle [191]. A dynamic household demands a satisfactory environment for family and children with better school, larger area for child rearing. While a young household would prefer independence and proximity to city centre. Clarke and Onaka (1983) attempt to improve Rossi's model on household life cycle and determined 18 triggers for moving in order to come up with a typology [192]. The "adjustment moves" were determined by housing characteristics, neighbourhood quality and accessibility to work, services, family, friends and the reason is shared among all age group [192]. The model was developed further to demonstrate the trade-off between workplace commute and access to services during the decision making process of an household. Kim et al. (2005) showed trade-off between 


\section{RESIDENTIAL CHOICE MODEL}

workplace accessibility and environmental factors in residential choice varies at different stages of the life cycle as they emphasised the significant role of children [193].

\subsubsection{Individual Decision-Making}

An individual's well being or quality of life depends on overall satisfaction of social, economic, and environmental needs as discussed in section 7.2.2.2. Each individual, despite their income group or social status, would attempt to maximize their overall satisfaction to the best of their ability. Suppose activity $A_{i}$ produces a certain amount of satisfaction level for an agent $(a)$ based on social, economic, and environmental needs which can be denoted as $S_{\mathrm{soc}}(\mathrm{a}), S_{\mathrm{eco}}(\mathrm{a})$, and $S_{\text {env }}$ (a) respectively. Now agent (a) may value different needs with varying level of importance (weights) denoted by $\omega_{\text {soc }}(\mathrm{a}), \omega_{\text {eco }}(\mathrm{a}), \omega_{\text {env }}(\mathrm{a})$ respectively. The value of each weight should be in the range $[0,1]$ and the sum of all the weights should be 1 i.e $\omega_{\text {soc }}(a)+\omega_{\text {eco }}(a)+\omega_{\text {env }}(a)=$ 1. Thus the overall satisfaction of agent $S_{\text {overall }}$ (a) can be formulated as the weighted sum of all the three needs as denoted below:

$$
S_{\text {overall }}(a)=\omega_{\mathrm{soc}}(a) * S_{\mathrm{soc}}(a)+\omega_{\mathrm{eco}}(a) * S_{\mathrm{eco}}(a)+\omega_{\mathrm{env}}(a) * S_{\mathrm{env}}(a)
$$

The satisfaction level of an agent $(a)$ depends on its current socio-economic status and personal expectations from the three needs. This can be formalized as:

$$
S_{m}(a)=f\left(P_{m}(a), R_{m}(a)\right)
$$

where $m$ represents either of social, economic and environmental dimension, $P$ and $R$ are respectively the state and reference points of each dimensions $(m)$.

While economic and social satisfaction follows a vector model, the environmental satisfaction is formalized according to the ideal point model. The vector model of satisfaction has two reference points $(R)$, namely the lower bound $R_{\min }$ and the upper bound $R_{\max }$ depending on individual's choice. The threshold values provide a range between which the satisfaction of an individual is linearly increasing and if the current state $P$ is below the lower bound $R_{\text {min }}$ the individual in completely unsatisfied. The satisfaction increases till the upper bound $R_{\max }$ beyond which the individual is completely satisfied. The vector model can be formulated as: 


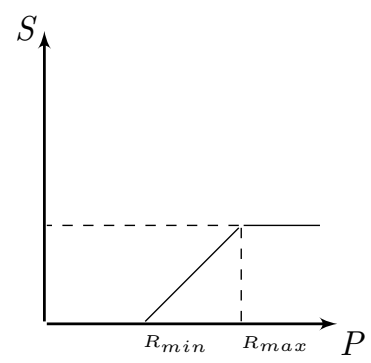

Figure 7.2: Vector model representation.

$$
f= \begin{cases}0 & \text { when } P \leq R_{\min } \\ \frac{P-R_{\min }}{R_{\max }-R_{\min }} & \text { when } R_{\min }<P<R_{\max } \\ 1 & \text { when } P \geq R_{\max }\end{cases}
$$

Environmental satisfaction $S_{\text {env }}($ a) expresses how satisfied an agent is with living in a slum, and can be used to calculate its expected social satisfaction when moving to another slum. For the urban-poor the environmental satisfaction might not be very significant. However, some households might not prefer to inhabit slums near sewage systems or railway tracks. Similar to the vector model, the ideal point model has three reference points $(R)$, namely the lower bound $R_{\min }$ and the upper bound $R_{\max }$. However, in the ideal point model an ideal point $R_{\text {ideal }}$, lies between the threshold values $R_{\min }$ and $R_{\max }$, where the satisfaction is at peak. The satisfaction is minimal outside the range $\left[R_{\min }, R_{\max }\right]$. The ideal point model can be formulated as:

$$
f= \begin{cases}1 & \text { when } P=R_{\text {ideal }} \\ 0 & \text { when } P \leq R_{\text {min }}, P \geq R_{\max } \\ \frac{P-R_{\text {min }}}{R_{\text {ideal }}-R_{\min }} & \text { when } R_{\text {min }}<P<R_{\text {ideal }} \\ \frac{R_{\text {max }}-P}{R_{\text {max }}-R_{\text {ideal }}} & \text { when } R_{\text {ideal }}<P<R_{\text {max }}\end{cases}
$$

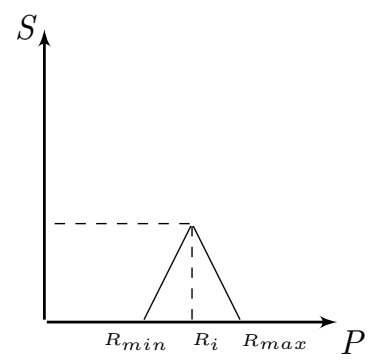

Figure 7.3: Ideal point model representation

Based on equation 7.8 to $7.10, S_{\mathrm{soc}}(\mathrm{a}), S_{\text {eco }}(\mathrm{a})$, and $S_{\text {env }}(\mathrm{a})$ has been formulated as the 


\section{RESIDENTIAL CHOICE MODEL}

function of $P$ and $R$ (respectively the current state and reference points of social, economic and environmental dimension). Therefore, the next steps are to formulate $P$ and $R$. The formulation of reference points $(R)$ is explained in section 7.2.3.2 and the formulation of current state $(P)$ is explained below (based on discussion in 7.2.2.1). The social state of an individual agent $\left(P_{s o c}(a)\right)$ is formulated as:

$$
P_{\text {soc }}(a)=\frac{N_{\text {slums }}(a)}{N_{\text {Size }}(a)}
$$

where $N_{\text {slums }}(a)$ is sum of homophily over the number of node neighbours living in the slum and $N_{\text {Size }}(a)$ is the sum of homphily over all node neighbours in the network of agent $a$. The concept of homophily and its formalization is discussed in the next section. Economic and environmental state of an individual agent $\left(P_{\text {eco }}(a), P_{e n v}(a)\right)$ is formulated as:

$$
\begin{gathered}
P_{\text {eco }}(a)=\frac{\operatorname{Inc}(a)}{H H_{\text {Size }}(a) * \operatorname{Distance}(a)} \\
P_{\text {env }}(a)=\frac{\operatorname{Rent}(a)}{H H_{\text {Size }}(a) * \operatorname{Inc}(a)}
\end{gathered}
$$

where $\operatorname{Inc}(a)$ is the household income, $H_{H_{\text {Size }}}(a)$ is the size of the household, Distance(a) is the commute distance and $\operatorname{Rent}(a)$ is the rent paid by the household.

\subsubsection{Individual Sensing}

Agent's influence each other by sharing information and influencing the satisfaction of other agents in their social network. Hence, it is important to develop a robust social network which is based on real life parameters. The theoretical notion of social networks emphasize the importance of homophily (similarity) as a key factor in the likelihood of being connected $[194,195]$. The individual's social network impacts with whom they communicate. The degree of communication in their social network depends on the strength of their relation (tie strength), the similarity between the two individuals (homophily) [196]. The individual's social network is highly correlated to a slum dwellers' access to the slum and various resources (such as water, electricity etc). Individuals with extensive network within slums tend to have more control 
over various resources of the slums and sell these resources at a profit to other slum dwellers [197, 198].

The social network can be constructed in three steps. Firstly, the key is to determine the number of close contacts that the individual is likely to interact with in daily life, this can be termed as "regular contacts". This should result in a fixed size network for an individual which gets updated when there is change in situation of the individual, e.g change in job, moving to another slum, or any changes in social context. Hence, the social network can be formalized as a dynamic system which reacts to changes in activity and rearranges itself accordingly. The following formalisation dictates the intractability of the agents. These parameters have been identified through an extensive literature review of social network analysis in slums and in general [199, 200, 201, 202, 172].

The degree of interactibility of two agents $(a$ and $b)$ can thus be formalised as:

$$
I(a, b)=\operatorname{Neighbour}(a, b)+\operatorname{Sector}(a, b)+\epsilon
$$

where,

$$
\text { Neighbour }(a, b)= \begin{cases}1 & \text { when } \operatorname{Slum}_{a}=\text { Slum }_{b} \\ 0 & \text { when Slum } \\ a & \neq \text { Slum }_{b}\end{cases}
$$

and,

$$
\operatorname{Sector}(a, b)= \begin{cases}1 & \text { when } O c c_{a}=O c c_{b} \\ 0 & \text { when } O c c_{a} \neq O c c_{b}\end{cases}
$$

and, $\epsilon$ is random variable between 0 to 1 .

If $\mathrm{I}(\mathrm{a}, \mathrm{b})$ is greater than 0.8 , agent $a$ and $b$ may form a network.

\subsubsection{Interaction}

Now, once the network is determined the second step is to formulate how often each agent will interact with other agents in its network and the level of information sharing that can happen between two agents in the network. This will be guided by the degree of homophily (similarity) between two agents which basically means that agents sharing a similar interest will exert greater social influence on each. "Similarity breeds connection" - the principle of homophily organizes social ties of each kind, comprising of marriage, friendship, work and other types of relationship. Homophily binds individuals' social networks based on age, religion, education, 


\section{RESIDENTIAL CHOICE MODEL}

occupation, language and gender in a way that it influences the sharing of knowledge and information, the social belief of an individual and the interaction they experience [172].

The degree of homophily can be formulated as the similarity based on the following six key attributes: same slum, occupation and income category, same language, religion and gender. These groupings are same as discussed in chapter 4 . Hence, for every agent $b$ in $a$ 's neighbour the degree of homophily can be calculated as:

$$
D(a, b)=\frac{\left|A t t r_{a} \cap A t t r_{b}\right|}{\left|A t t r_{a}\right|}
$$

where $A t t r_{a}$ and $A t t r b$ is a set of node attributes of agent $a$ and $b . D(a, b)$ varies from 0 (no homophily) to 1 (high homophily). Therefore the probability that an edge exists between agent $a$ and $b$ can be formulated as:

$$
P_{\text {edge }}(a, b)=D(a, b) * \frac{k_{b}}{\sum_{j} k_{j}}
$$

where $k_{b}$ is the degree of agent $b$ normalized by the all pre-existing nodes (i.e. the denominator results in twice the current number of edges in the network). Therefore, the agents have a "preference" to attach themselves to similar (high homphily) agents with a probability that is proportional to the number of links that the existing agents already have. The initial degree distribution (figure 7.4) shows that the resultant network is scale free.

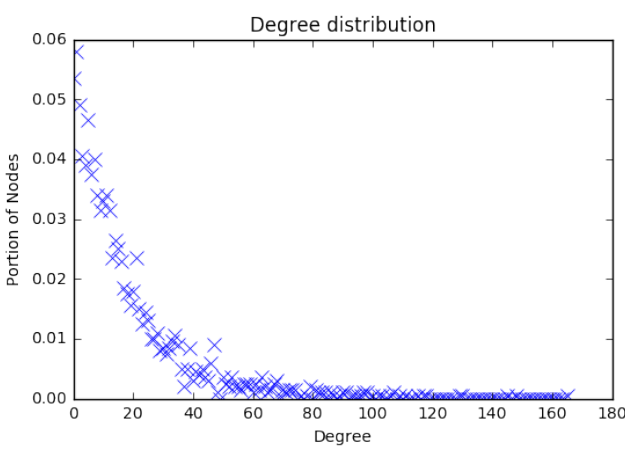

Figure 7.4: Actual Degree Distribution based on 1107 households from 37 slums in Bangalore.

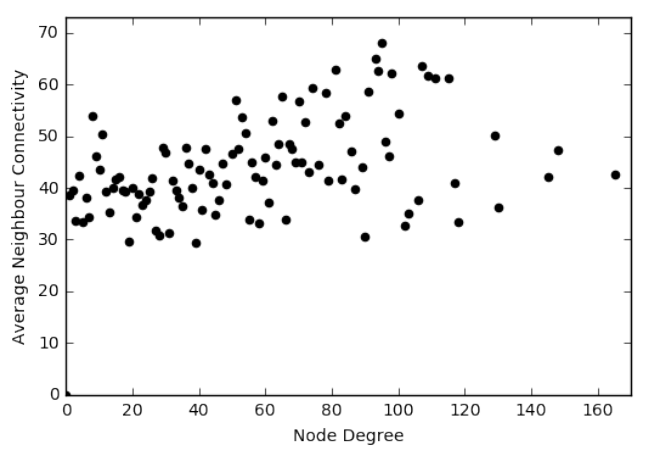

Figure 7.5: Actual Average Connection degree based on 1107 households from 37 slums in Bangalore.

The third step would be to rank all the neighbours of a given agent $(a)$ in the network based on the degree of homophily and derive the first $N$ agents as the most influential agents. The 
value of $N$ depends on the size of the network and the computational resources, and can be derived similar to Dunbar number [203]. Finally, at each time step it is important to update the social network and other state variables of each agent as it changes activities: residence or job.

\subsubsection{Individual Prediction}

A completely satisfied individual will continue with the current state and is less likely to change. The more the satisfaction decreases with respect to the need that is weighted more, chances of changing the activity increases for the individual. For example in the case of a slum dweller, the individual will be more likely to migrate to another slum as it provides an opportunity for higher income even though he might have fewer social connections. In such a situation, economic need will prevail over social and environmental needs. It may also be possible that a slum dweller is completely satisfied with his income and might be willing to pay more for better living condition in slums. Thus in the model the probability with which an agent $a$ updates its information can be formalized as:

$$
P_{\text {update }}(a)=1-S_{\text {overall }}(a)
$$

$P_{\text {update }}$ ranges from 0 to 1 , where 0 represents a fully satisfied agent who does not require to update any information while a value of 1 represent a completely dissatisfied agent who needs to update information at each time step. Information gathering can be achieved by either interacting with other agents in the social network or by exploring the available options by the agent itself. For example, when an agent $a$ who is currently not satisfied with the outcome of his activity comes in contact with agent $b$ who is highly satisfied with its current situation, agent $a$ will try to follow the same activity as $b$ with a willingness $\left(P_{\mathrm{a}, \mathrm{b}}\right)$ that can be formulated as:

$P_{\mathrm{a}, \mathrm{b}}=\omega_{\mathrm{soc}}(a) *\left(S_{\mathrm{soc}}(b)-S_{\mathrm{soc}}(a)\right)+\omega_{\mathrm{eco}}(a) *\left(S_{\mathrm{eco}}(b)-S_{\mathrm{eco}}(a)\right)+\omega_{\mathrm{env}}(a) *\left(S_{\mathrm{env}}(b)-S_{\mathrm{env}}(a)\right)$

If $P_{\mathrm{a}, \mathrm{b}}$ is greater than 0 , then agent $a$ 's interest of adopting $b$ 's activity (supposing $A c t_{j}$ ) is:

$$
P_{\text {Act }}(j)=P_{\text {update }}(a) * P_{\mathrm{a}, \mathrm{b}}
$$


Similarly, the agent $a$ 's willingness $\left(P_{\text {ind }}\right)$ to switch to activity $A c t_{j}$ based on individual processing can be formulated as:

$P_{\mathrm{ind}}=\omega_{\mathrm{soc}}(a) *\left(S_{\mathrm{soc}}(a)^{e}-S_{\mathrm{soc}}(a)\right)+\omega_{\mathrm{eco}}(a) *\left(S_{\mathrm{eco}}(a)^{e}-S_{\mathrm{eco}}(a)\right)+\omega_{\mathrm{env}}(a) *\left(S_{\mathrm{env}}(a)^{e}-S_{\mathrm{env}}(a)\right)$

where $S_{\mathrm{soc}}(a)^{e}, S_{\mathrm{eco}}(a)^{e}$ and $S_{\mathrm{env}}(a)^{e}$ denotes the estimated satisfaction from taking $A c t_{j}$.

Finally, agent $a$ 's interest of changing to $A c t_{j}$ is:

$$
P_{\text {Act }}(j)=P_{\text {update }}(a) * P_{\text {ind }}
$$

The $P_{\text {Act }}(j)$ derived in equation 7.21 and 7.23 are mutually exclusive. The residential choice model is designed to realistically represent the behaviour of slum dwellers in determining their choice of slum.

\subsubsection{Collectives}

Each agent is associated to a household and maintains a list of social network with which an agent can interact. Besides this, there are no collectives in the model.

\subsubsection{Heterogeneity}

The heterogeneity in agents is formalized by using personalized weights concerning the relative importance of economic, social and environmental $\left(\omega_{\mathrm{soc}}(\mathrm{a}), \omega_{\text {eco }}(\mathrm{a})\right.$ and $\left.\omega_{\text {env }}(\mathrm{a})\right)$ on their overall satisfaction level.

\subsubsection{Stochasticity}

The decision making in the model is completely stochastic as explained above. The initial spatial distribution also follows Complete Spatial Randomness (CSR) distribution.

\subsubsection{Details}

In this section, we describe the implementation of the residential choice model in Dynaslum with respect to the slums in Bangalore. The focus is on how field data, empirical evidence, and theoretical-grounded constructions are integrated in the agent based model. 


\subsubsection{Agent Population}

As a first step we have to construct a population of agents that reflects the demography in the slums of Bangalore. For this we used the data collected from 37 slums in Bangalore (explained in chapter 4). The spatial part of the model is initialized, using a random distribution of households living in each slums along with their family members.

An individual's working status can be either worker or unemployed. For those individuals whose working status is 'worker', information is generated about their occupation category and the commuting distance (as the distance between a worker's slum and the job site). Furthermore, we also generate individual's skill (initialised as a occupation code) and income.

\subsubsection{Estimating Agents' satisfaction levels}

In order to compute an agent's individual satisfaction values, the following two sets of parameters needs to be estimated:

\section{- Reference points}

1. Social Satisfaction: The $\operatorname{Soc}_{\min (a)}$ is the 20 percentile level of the distribution of $S o c_{a}$, while $S o c_{\max (a)}$ is the 80 percentile level of the distribution of $S o c_{a}$.

2. Economic Satisfaction: The threshold values of economic satisfaction are estimated based on distribution of income in an given occupation category. The $E c o_{\min (a)}$ and $E c o_{\max (a)}$ are the 20 percentile and 90 percentile income respectively derived from the data described in chapter 4 .

3. Environmental Satisfaction: The threshold values of environmental satisfaction are estimated based on distribution of per capita rental values from the data described in chapter 4. The $E n v_{\min (a)}, \operatorname{Env}_{\text {ideal }(a)}$ and $E n v_{\max (a)}$ are the 20 percentile, 50 percentile and 90 percentile rental values respectively. In case of house owners, the environmental satisfaction is set high, at a arbitrary level of 0.95 .

\section{- Personal Preference}

$\omega_{s o c}, \omega_{e c o}, \omega_{\text {env }}$ are respectively the weights that represents the importance of each dimension in the satisfaction level of individual agents. This allows formalizing heterogeneity in agents concerning the relative importance of economic, social and environmental outcomes on their over-all satisfaction level. To formalize the heterogeneity in satisfaction mathematically, we would like to determine the probability density of every possible value 
of $\omega$ for a given household in the simulation. Therefore, we consider each component of $\omega$ as being an independent variable. In this case, for $\omega=\left\{\omega_{s o c}, \omega_{e c o}, \omega_{e n v}\right\}$ we assume $\omega_{s o c}$, $\omega_{e c o}, \omega_{e n v}$ as independent variables and $\omega$ as a vector in 3 dimensional space. Applying the constraint of multinomial distribution:

$$
\omega_{s o c}+\omega_{e c o}+\omega_{e n v}=1
$$

we can say that allowed values of $\omega$ must lie in a plane. Furthermore, since $\omega_{i} \geq 0$, values of $\omega$ is limited to an equilateral triangle (a 2-simplex) as shown in figure 7.6.

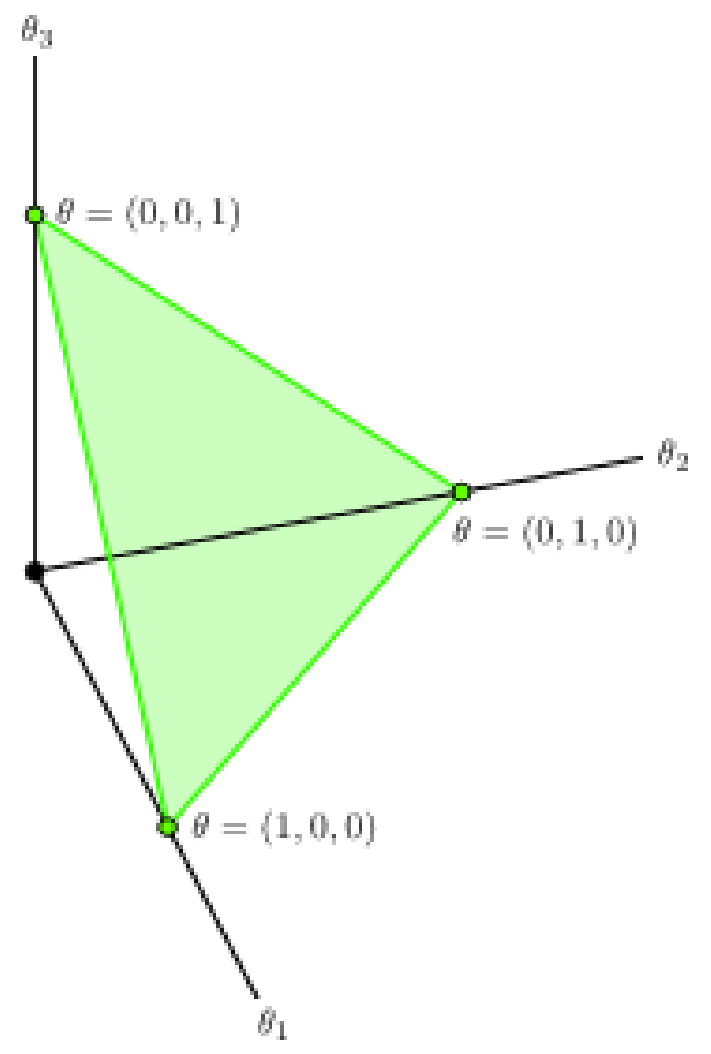

Figure 7.6: A 2- Simplex showing the possible values of $\omega$

We use the Dirichlet distribution to determine the probability density for our multinomial parameter $(\omega)$. The Dirichlet distribution takes the form:

$$
\operatorname{Dir}(\alpha) \rightarrow p(\omega \mid \alpha)=\frac{\Gamma(\alpha)}{\Gamma\left(\alpha_{0}\right) \Gamma\left(\alpha_{L}\right)} \omega_{0}^{\alpha_{0-1}} \times \ldots \times \omega_{\Gamma}^{\alpha_{L-1}}
$$


where

$$
\left\{\omega_{i}\right\} \geq 0 ; \sum_{i=1}^{L} \omega_{i}=1
$$

and

$$
\begin{gathered}
\alpha \geq 0 ; \\
\sum_{i=0}^{L} \alpha_{i}=\alpha
\end{gathered}
$$

where Gamma function $\Gamma(k)$ is a continuous variant of the factorial function. The Dirichlet Family generalizes the Beta family to a vector $\omega=\left(\omega_{0}, \omega_{1}, \ldots \omega_{L}\right)$ in which $\sum_{i=0}^{L} \omega_{i}=1$ and the $\left\{\omega_{i}\right\}$ are non-negative. Here, $\omega$ describes the outcome variable, the $L$-tuple, the one for which we want to calculate probability. The shape of the probability model is determined by $L$ shape parameters, $\left(\alpha_{1}, \alpha_{2}, \ldots, \alpha_{L}\right)$. These shape parameters are used to "tune" the distribution, to make certain $L$-tuples more likely than others. As the illustrated in figure, the large values of $\alpha_{i}$ correspond to actions which make outcome $i$ "more likely". In our case, $L=3$ and $p(\omega \mid \alpha)$ determines "the probability density associated with multinomial distribution $\omega$, given that the Dirichlet distribution has parameter $\alpha . "$

As shown in figure $7.7, \alpha=(1,1,1)$ yields a uniform distribution, where all points on the simplex are equally probable. For values of $\alpha_{i} \leq 1$, the distribution concentrates in the corners and along the boundaries of the simplex. The colour scale runs from dark blue (lowest values) to red (highest values). For values of $\alpha_{i} \geq 1$, the distribution tends toward the centre of the simplex. As $\alpha_{i}$ increases, the distribution becomes more tightly concentrated around the centre of the simplex. Finally, for a symmetric Dirichlet with $\alpha_{i} \geq 1$ and similar $\alpha_{i}$ will denote equal weights for social, economic and environmental satisfaction. However, when we need to assign higher weight to environmental satisfaction, we would want an asymmetric (non-central) Dirichlet distribution with a higher value for $\alpha_{3}$.

\subsection{Validation and Sensitivity Analysis}

In this section, we use the case study of the city of Bangalore in India to validate the Residential Choice Model. We also present various scenarios under which the model is validated and discuss the impact of economic, social and environmental on residential mobility of slum dwellers. 
$\alpha=(0.999,0.999,0.999)$

$\alpha=(5.000,5.000,5.000)$

$\alpha=(25.000,5.000,2.000)$
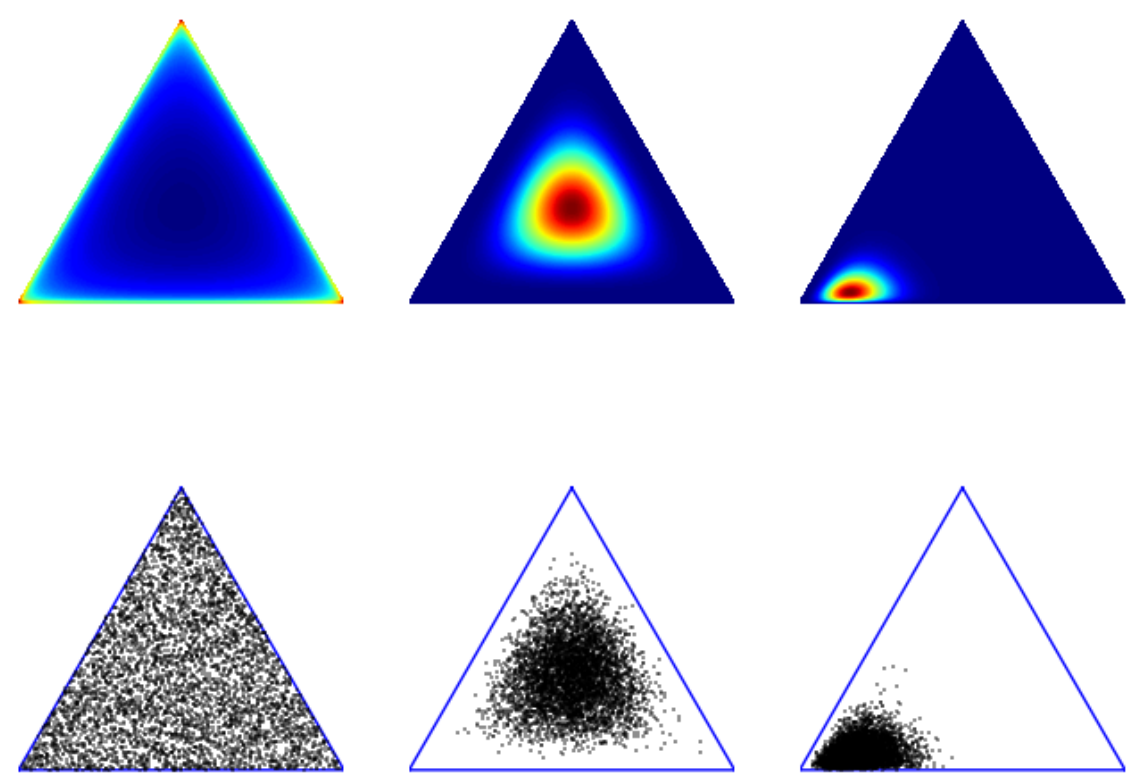

Figure 7.7: A ternary diagram showing the possible distribution of $\omega$ using Dirichlet distributions.

\subsubsection{Constant Population and Constant Labour Market}

In this section we present the results of validation in the residential choice model assuming a constant population and labour market. In other words, new households are not created in the simulation and job scenario is also static. The initial households are drawn from the 37 slums in Bangalore (described in chapter 4). The households are then randomly distributed across these 37 slums in Bangalore. This scenario helps us to understand the impact of economic, social and environmental satisfaction on mobility of slum households in isolation. The objective is to understand, given a random initial spatial distribution of households, and with all factors being constant, how and if the system attain a steady state comparable to the data collected from the slums in Bangalore.

Social mobility is measured by the number of moves by all households in the model at a given time step. The model was executed using the following four configurations: 


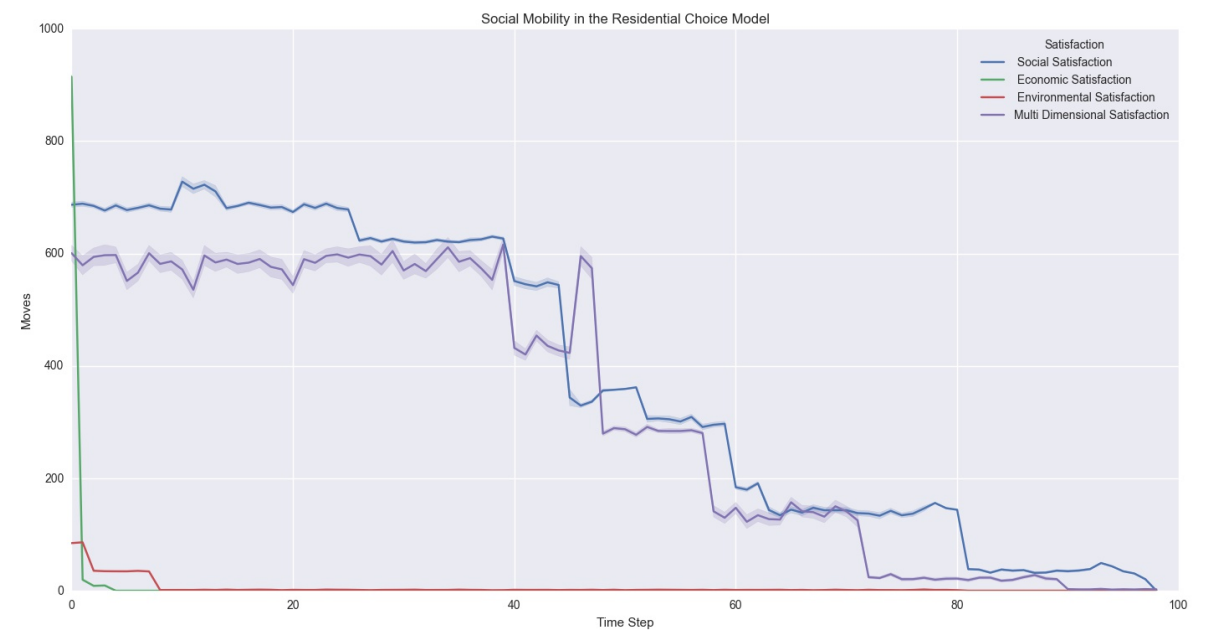

Figure 7.8: Social Mobility of the Residential Choice Model under Constant population and Constant Labour Market.

1. Only Social Satisfaction i.e. $\omega_{s o c}=1, \omega_{e c o}=0, \omega_{e n v}=0$

2. Only Economic Satisfaction i.e. $\omega_{s o c}=0, \omega_{e c o}=1, \omega_{e n v}=0$

3. Only Environmental Satisfaction i.e. $\omega_{\text {soc }}=0, \omega_{\text {eco }}=0, \omega_{\text {env }}=1$

4. Multi-dimensional Satisfaction i.e. $\omega_{s o c}, \omega_{e c o}, \omega_{e n v}$ are drawn from Dirichlet distribution as described in previous section.

Each configuration was executed 20 times until the model reached a steady state (the number of moves remained constant for 10 time steps). Figure 7.8 shows that when only social satisfaction is included in model, the number of moves increases as all household agents attempts to maximize their social satisfaction. As expected, the number of moves gradually decreases and reach a steady state once all households have maximised their social satisfaction. In comparison, when the model is run only with economic and environmental satisfaction, the households quickly organize to attain steady state. Therefore, figure 7.8 indicates that although the population is constant, a dynamic social network could lead to considerable migration among slum dwellers.

Figure 7.8 also shows the number of moves in the model when social, economic and environmental satisfaction are included in the model. At around $t=80$, most households reach a steady state (maximum satisfaction). Table 7.4 shows the segregation of the households with 


\section{RESIDENTIAL CHOICE MODEL}

\begin{tabular}{|l|l|l|l|l|l|l|}
\hline Factors & G1 & G2 & $\omega_{\text {soc }}=1$ & $\omega_{\text {env }}=1$ & $\omega_{\text {eco }}=1$ & $\omega_{\text {soc,eco,env }} \neq 0$ \\
\hline Gender & Male & Female & $0.11(0.02)$ & $0.13(0.06)$ & $0.11(0.02)$ & $0.019(0.03)$ \\
\hline Language & Southern & Others & $0.32(0.07)$ & $0.23(0.12)$ & $0.04(0.01)$ & $0.578(0.18)$ \\
\hline Religion & Hindu & Muslim & $0.45(0.13)$ & $0.21(0.13)$ & $0.32(0.09)$ & $0.757(0.25)$ \\
\hline Income & Low & High & $0.27(0.07)$ & $0.15(0.11)$ & $0.68(0.18)$ & $0.434(0.12)$ \\
\hline Occupation & Formal & Informal & $0.34(0.11)$ & $0.12(0.09)$ & $0.13(0.05)$ & $0.404(0.17)$ \\
\hline
\end{tabular}

Table 7.4: Comparison of Index of Dissimilarity calculated based on the simulation output under 4 different condition: $\omega_{\text {soc }}=1, \omega_{e n v}=1, \omega_{\text {eco }}=1, \omega_{\text {soc }}+\omega_{\text {eco }}+\omega_{\text {env }}=1 \in \operatorname{Dir}(\alpha)$

respect to religion. We find that the observed patterns in the model are comparable to the spatial segregation in the actual data where Muslim majority slums form one cluster while Hindus are distributed across all slums in Bangalore. Similarly, the language segregation in the residential choice model (shown in table 7.4) is similar to observed patterns in the data discussed in chapter 4 . Therefore, this comparison validates that the rules driving the model are realistic and could be used to conduct What-If experiments.

\subsubsection{Increasing Population and Constant Labour Market}

In this section we present the results of the residential choice model assuming a growing population but constant labour market. Therefore, new households are created in the simulation at each time step but the job scenario is static. In this case, the initial condition is taken from the steady state achieved in the previous section. At each time step, $0.2 \%$ households are added, their profiles (state variables) drawn at random from the data collected from 37 slums in Bangalore. This scenario helps us to validate the impact of population growth and migration on social mobility of slum households. The objective is to understand, given a steady but small increase in population, how does it impact the satisfaction of existing households and emergence of slums in Bangalore. The configuration was executed 20 times for 100 time steps. Figure 7.9 shows that initially, the number of moves increases as new households are created. However, at around $t=5$, we find that there is a spike in the number of the moves indicating that the system undergoes a critical transition at a certain threshold value of the population. Further analysis indicates that with addition of each household the satisfaction value changes. However, this change is small until a threshold population is reached, at which point a large number of population reorganize to maximise their satisfaction. As expected, the number of moves gradually decreases and reach a steady state once all households have maximised their combined satisfaction. We also find that the behaviour is periodic as shown in figure 7.9. 


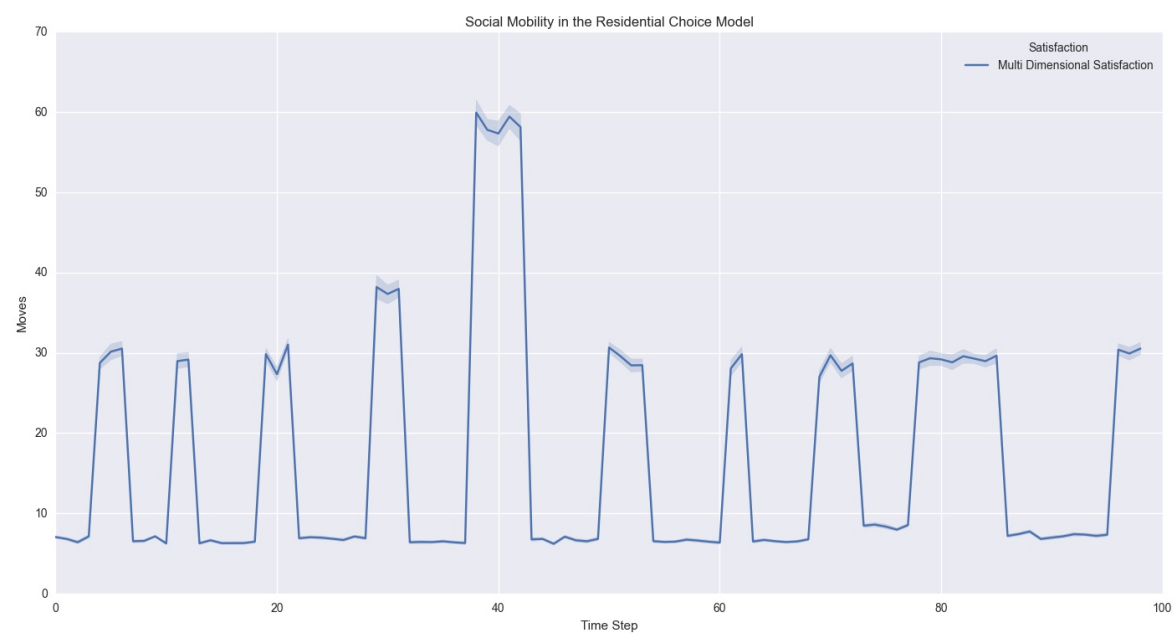

Figure 7.9: Social Mobility of the Residential Choice Model under Dynamic population and Constant Labour Market.

\subsubsection{Increasing Population and Dynamic Labour Market}

In this section we present the results of the residential choice model assuming a growing population and a dynamic labour market. Therefore, new households are created in the simulation at each time step. Similarly new jobs are created and old jobs are expired to simulate a dynamic labour market. In this case, the initial condition is taken from the steady state achieved in the previous section. At each time step, $0.2 \%$ households are added, their profiles (state variables) drawn at random from the data collected from 37 slums in Bangalore. At each time step 2 new slums are created at a random location. This scenario helps us to validate the impact of population growth and migration along with a dynamic informal labour market on social mobility of slum households. The objective is to understand, the effect of dynamic labour market on the growth and emergence of slums in Bangalore.

The configuration was executed 20 times for 100 time steps. Figure shows that initially, the number of moves increases as new households and jobs are created. However, at around $t=18$, we find that there is a spike in the number of the moves indicating that the system undergoes a critical transition at a certain threshold value of the population. Further analysis indicates that with addition of each household the satisfaction value changes. However, this change small until a threshold population is reached, at which point a large number of population reorganize to maximise their satisfaction. The behaviour is similar to the pattern observed in figure 7.9, 


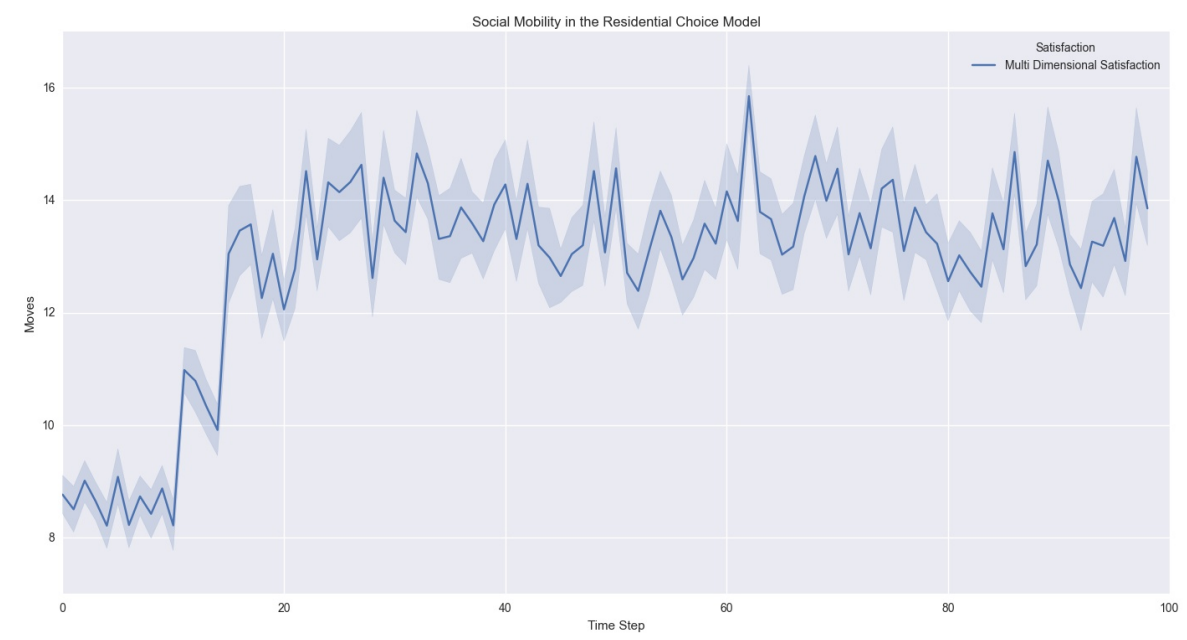

Figure 7.10: Social Mobility of the Residential Choice Model under Dynamic population and Dynamic Labour Market.

however the system do not return back to a oscillatory state. Instead the interaction between new households and a dynamic job creation keeps the system in a dynamic state where economic mobility of households leads to social mobility which in turn changes the social network and thus changing the social satisfaction.

Finally, we also compare the observed and simulated distributions for commute distance of the households. The observed mean commute distance is 5.02 kilometres (kms) compared to $5.78(0.43)$ in the simulation. Figure 7.11 and 7.12 demonstrates that the fit of the observed and simulated distributions is reasonable. According to the Kolmogorov Smirnov test, these two distributions are the same (50.476).

The findings from the distribution of commute distance, which is completely an emergent phenomenon in the model emphasize the trade-off between commute and economic opportunities. It also demonstrates that slum dwellers may employ spatial mobility (relocation to different slum) as a coping strategy for increasing commute. The data for the slums and the simulation in Bangalore provide another confirmation of the importance of a critical distance, in this case about $10 \mathrm{kms}$, beyond which the likelihood of decreasing the distance to work grows rapidly, primarily by relocating close to the workplace.

The final is set of validation was done by comparing the simulated network structure and the initial structure (figure 7.4 and 7.5) from data. As shown in figure 7.13 and 7.14 the pattern of 


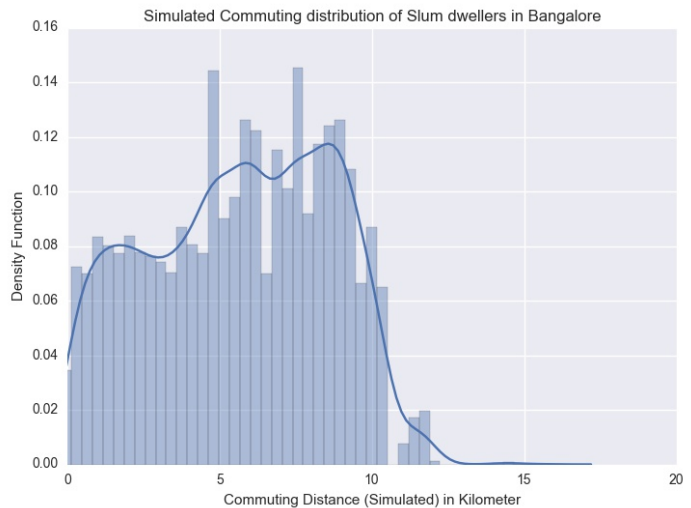

Figure 7.11: Simulated Distribution of Commuting Distance.

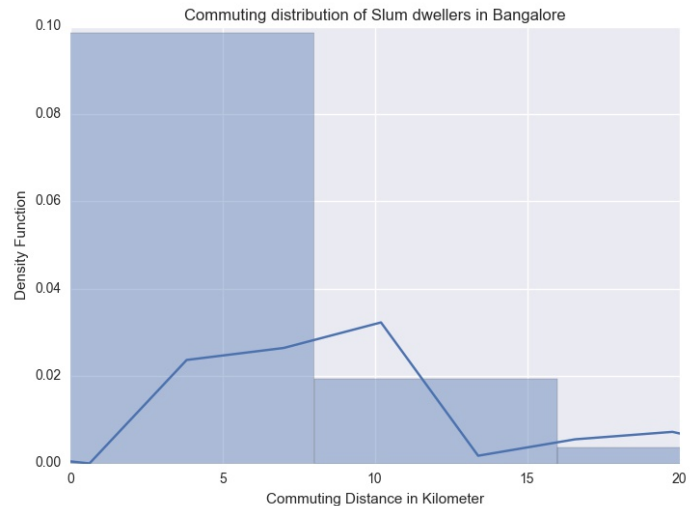

Figure 7.12: Observed Distribution of Commuting Distance (1107 slum households).

degree distribution (scale free) and average connection is similar to observed data. This shows that the network structure is retained in the simulation.

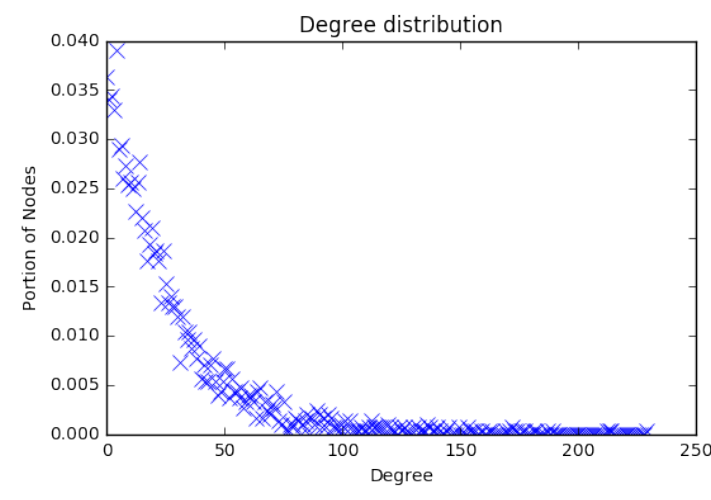

Figure 7.13: Simulated Degree Distribution.

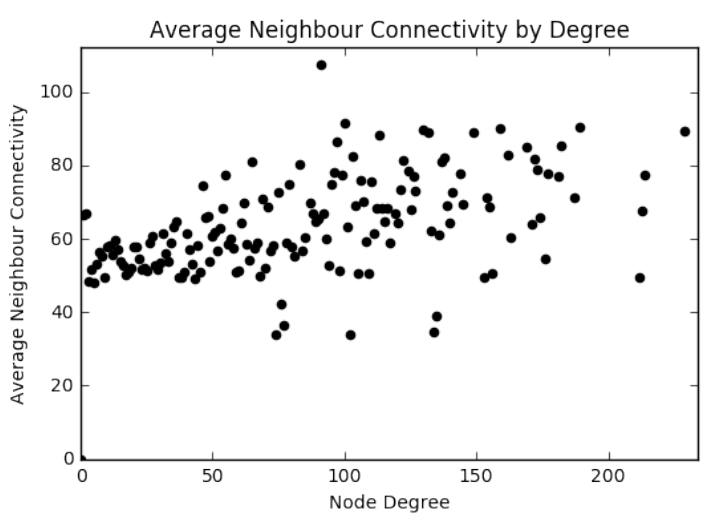

Figure 7.14: Average Connection degree.

\subsubsection{Sensitivity analysis}

In this section we describe the sensitivity analysis (SA) of the residential choice model. We perform the SA to identify important parameters and to characterize their influence on the social and economic mobility of the simulated population. In this case we focus on the parameter vector $\omega=(\omega s o c, \omega e n v, \omega e c o)$ to understand how it influences the model output, which is measured by the Dissimilarity index, $D$, calculated based on gender, religion, language and occupation. Inferences are based on a prior Dirichlet distribution $\operatorname{Dir}(\alpha)$ (see section 7.2.3.2), 
meant to capture heterogeneity in the model parameters, and the observed computer model output. In the SA we use 60 uniform samples of $\omega$ scaled from 0 to 1 . The analysis indicates

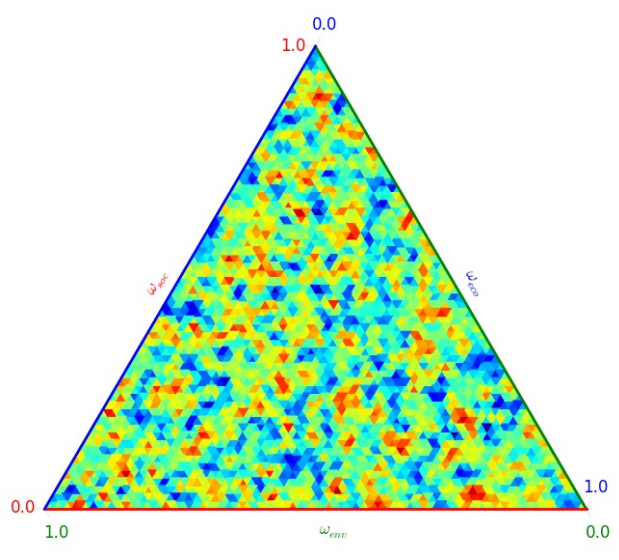

Figure 7.15: SA showing the impact of the parameter $\omega$ on Gender segregation.

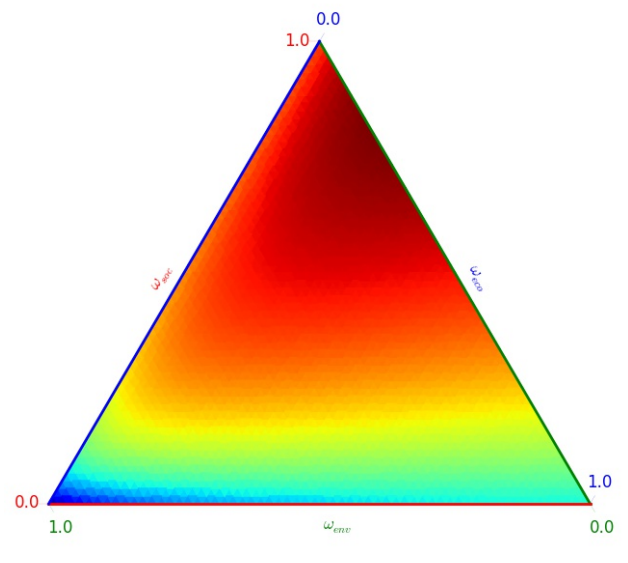

Figure 7.17: SA showing the impact of the parameter $\omega$ on Language segregation.
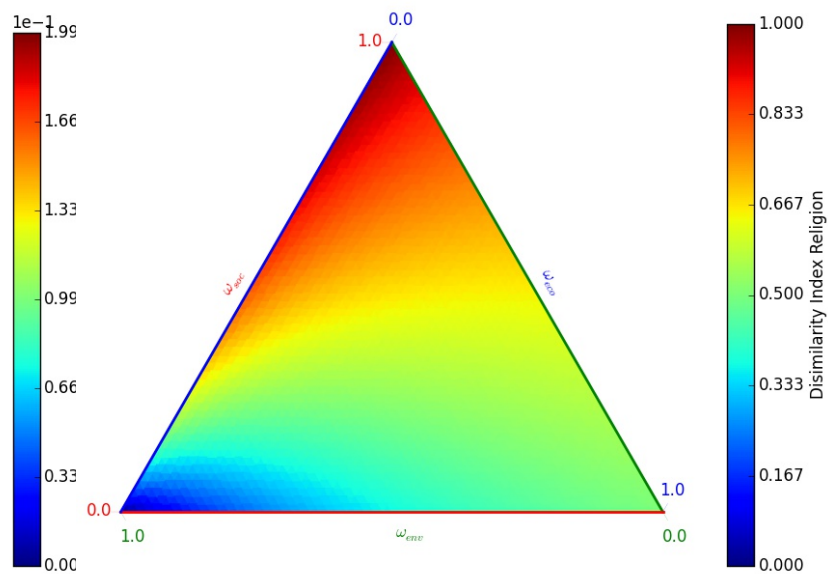

Figure 7.16: SA showing the impact of the parameter $\omega$ on Religion segregation.
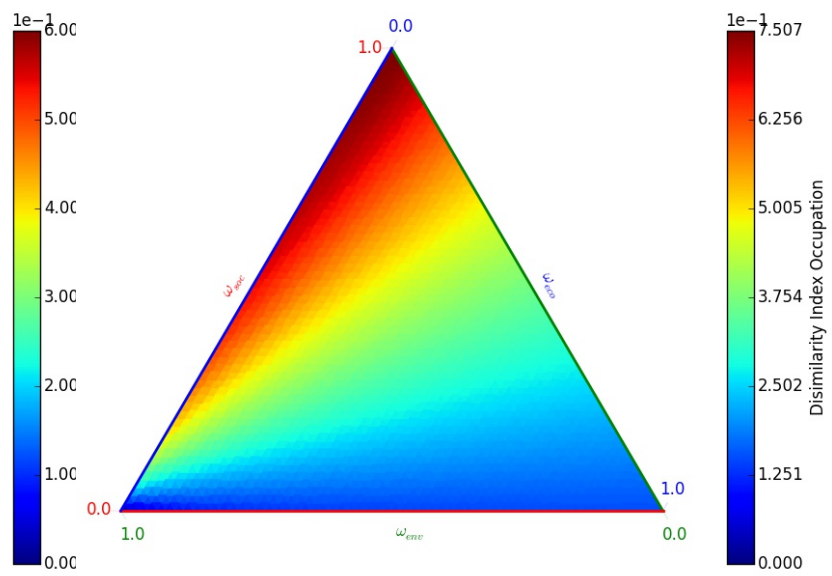

Figure 7.18: SA showing the impact of the parameter $\omega$ on Occupation Bias.

that increasing the $\omega_{\text {soc }}$ can favour social segregation depending on the value $\omega_{\text {eco. }}$. Figure 7.16 to 7.18 indicates that $\omega_{e n v}$ has little impact on the social and economic mobility in the model. This may be due to the fact the underlying conditions (rent distribution) of the slums in Bangalore are similar across the city. Therefore, even a high $\omega_{\text {env }}$ will lead to a random uniform distribution of the population, leading to a low Dissimilarity index. On the other hand we observe that high $\omega_{s o c}$ leads to high segregation based on religion, language and occupation (see figure $7.16,7.17,7.18$ ); thus conforming the findings of chapter 5 that spatial segregation 
based on religion and language leads to opportunity bias.

\section{$7.4 \quad$ Results}

In this section, we present and discuss simulation results of the residential choice model. As discussed in section we start with a baseline scenario using the survey data. In the simulation the population of the households increase at $0.2 \%$ per time step, jobs are randomly created from the distribution at each slum locations and two new slum location can be created per time step. The results are presented to demonstrate that the model can capture dynamic and complex interactions at a lower spatial scale. We also show the effect of social satisfaction on a dynamically changing environment.

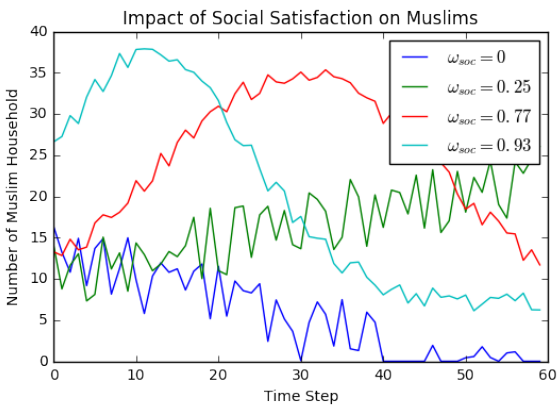

Figure 7.19: Impact of Social Satisfaction using Residential Mobility on Muslims.

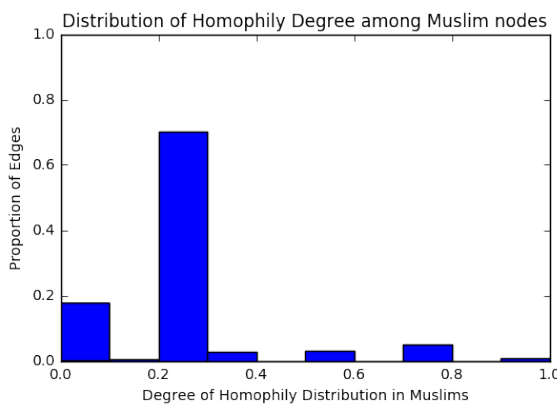

Figure 7.21: Distribution of Homophily Degree among Muslims.

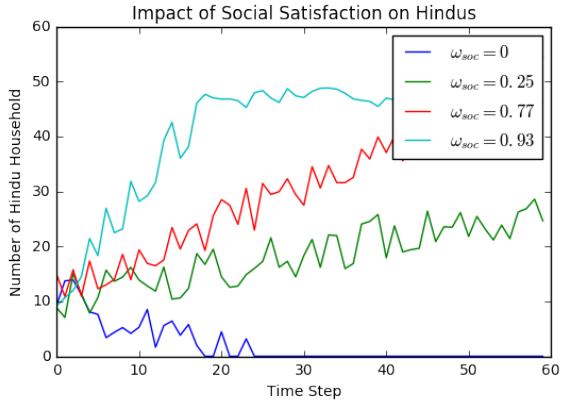

Figure 7.20: Impact of Social Satisfaction using Residential Mobility on Hindus.

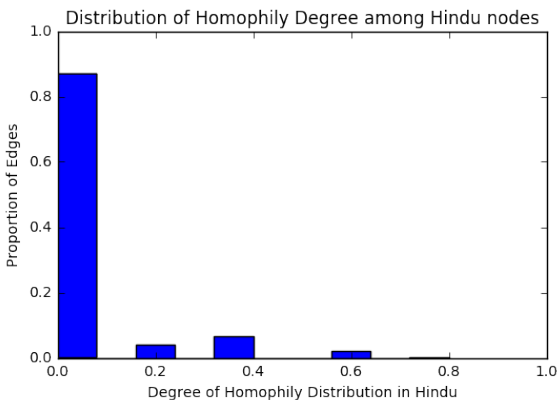

Figure 7.22: Distribution of Homophily Degree among Hindus. 


\subsubsection{The Effect of Social Satisfaction on Residential Choice}

In the residential choice model, social satisfaction determines the probability of an agent living in the same slum as the agent's social network and is formalized as the proportion of an agent's social contacts that reside in the same slum. When the social satisfaction is high, it may cause agents to reside in the same slum despite lower economic and environmental satisfaction, and thus stabilize the population to a certain extent due to a positive social feedback. However, if the social satisfaction continues to decrease due to the lesser number of social contacts, this may trigger a cascading effect where more agents leave the slum. In case of a minority social group in a slum (such as Hindus in a Muslim majority slum), agents that are reasonably satisfied may leave due to a decrease in social satisfaction, providing a negative feedback in the system triggering a rapid decrease in population, thus destabilizing a population.

To understand this behaviour, we ran the model using different $\omega_{s o c}$ in a Muslim and Hindu majority slum and observed the change in population over a period of time. Figure 7.19 shows that at $\omega_{s o c}=0$ (no social satisfaction), a depopulating slum would rapidly lose its population. However, as $\omega_{s o c}$ increases it stabilizes the population until it reached a critical value $\omega_{s o c}=0.77$ at which the social satisfaction is so important that it triggers a cascading effect leading to drastic drop in population. In population ecology terms, as $\omega_{s o c}$ increases, we find a transition from a extinction threshold $\left(\omega_{s o c}=0\right)$ to positive density dependence $\left(\omega_{s o c}=0.25\right)$ to density independence $\left(\omega_{s o c}=0.77\right)$. However, as shown in figure 7.20 the Hindu households behave differently. At $\omega_{s o c}=0$ (no social satisfaction), the slum would rapidly lose its population. However, as $\omega_{s o c}$ increases it stabilizes the population but do not reach a critical point which triggers a cascading effect as observed in figure 7.19. To analyse this difference in behaviour we study the distribution of the degree of homophily among Hindus and Muslims. Comparing figure 7.21 and 7.22 we observe that most Hindu households have low degree of homophily in their network (0-0.1) as compared to Muslim households. Theerfore, the proabibility of higher decrease in social satisfaction due to a "node" leaving the network of a Muslim household is significantly higher than that of Hindu households. Further, to understand the difference in degree of homophily among Hindus and Muslims we analyse equation 7.17. We find that opportunity bias and language distribution among Muslims creates high degree of homophily or "stronger ties". Figure 7.23 shows that occupation categories 4 to 6 are clearly dominated by Muslims. Similarly, figure 7.24 shows that most Muslims speak Urdu (7). This in turn means that when forming a network for a Muslim node, the probability that the node will have a higher degree of homophily with other nodes in the network is higher that of a Hindu node. 
The findings relates to 5 where we have shown how opportunity bias and segregation reinforces each other.

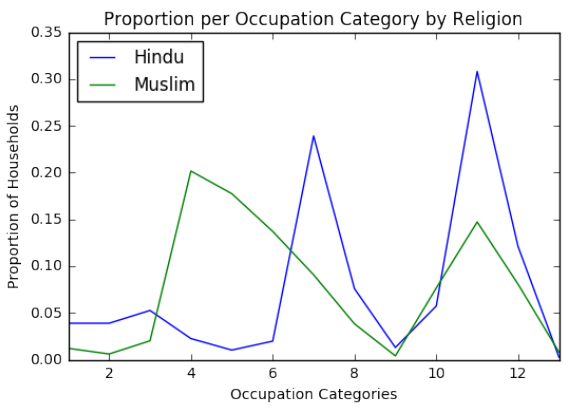

Figure 7.23: Distribution of Occupation Categories.

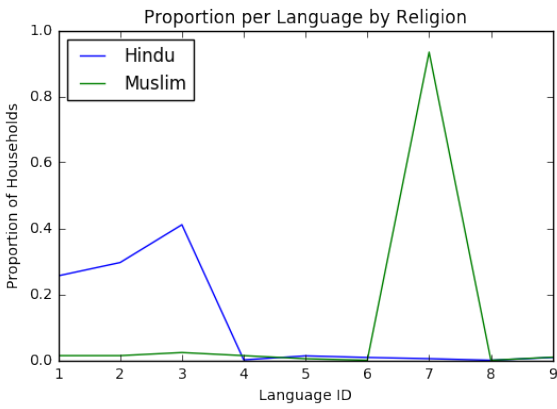

Figure 7.24: Distribution of Languages.

These findings have implications on strategic policy interventions which deals with population conservation or dispersion. It provides insights for policy makers to understand why certain slums may be more resilient to resettlement or evictions.

\subsubsection{The Effect of Jobs on Residential Choice}

In this section, we try to recreate a real scenario, from Hoodi in Bangalore where migratory growth patterns were observed for a slum as shown in figure 7.29. Figure 7.25 to 7.28 shows that the residential choice model is able to recreate the scenario. The scenario is recreated by modifying the underlying job scenario in the model. At time $t=0$, we observe a single slum $i$ in the simulation (figure 7.25). The job generation model was customized to expire (purge) all jobs in the slum $i$ and create new jobs at different location. At $t=8$ (figure 7.26) we observe that population size at slum $i$ decrease while a new slum $j$ is formed nearby. At $t=12$, new but different jobs are created at slum $i$ (figure 7.27), which leads to migration back to the slum as shown in figure 7.28.

In case of residential areas, integrated residential development should be attempted taking into account the functional need and socio-economic situation of the population. In different stages of the development, phased use of common space can be arranged. In the first stage, space is used for temporary accommodation of construction workers and a few hawkers and petty traders who cater to the need of the construction workers. Subsequently when the residential apartment is complete, construction workers may move out of the area to find other economic opportunities in the city. Once, the newly built residential complex is inhabited by higher 


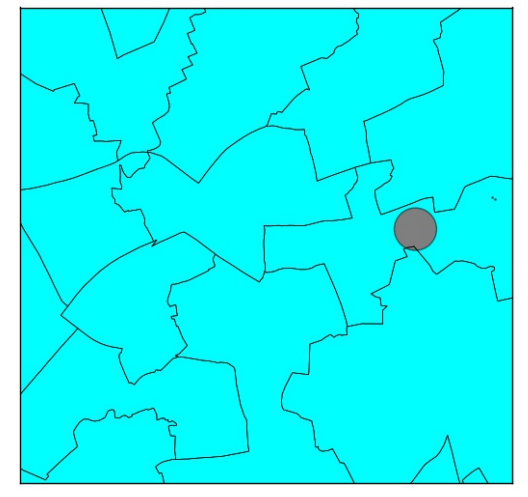

Figure 7.25: Simulation Slum Migration: State of a slum at $t=0$. The size of the circle represents the number of slum dwellers in the slum.

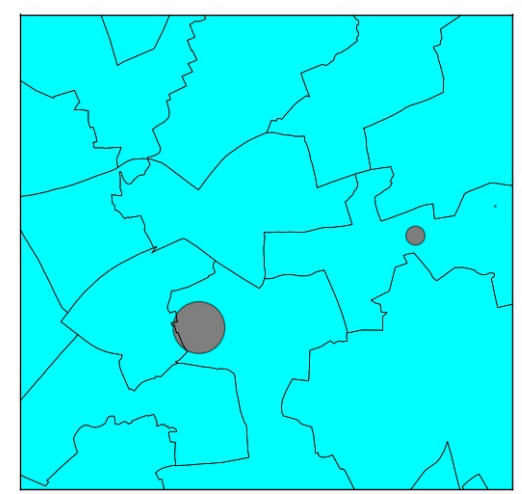

Figure 7.27: Simulation Slum Migration: State of slum at $t=12$. Slum dwellers migrate (due to job loss) and form a new slum nearby for new oppurtunities.

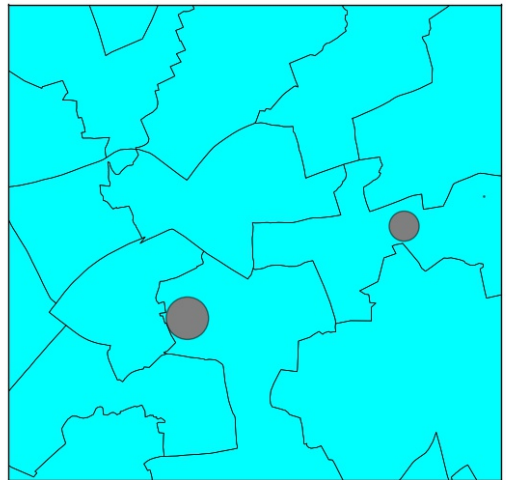

Figure 7.26: Simulation Slum Migration: State of slum at $t=8$. Slum dwellers migrate (due to job loss) and form a new slum nearby for new opportunities

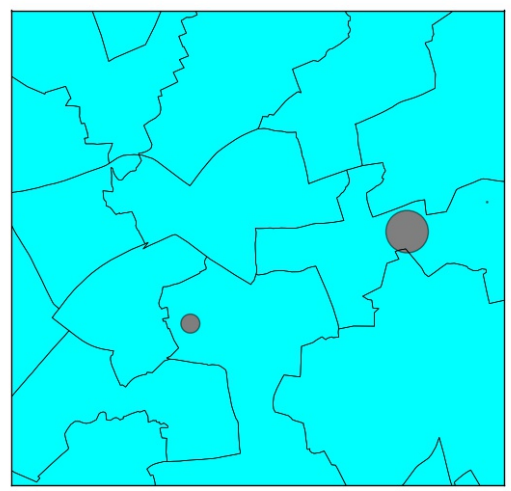

Figure 7.28: Simulation Slum Migration: State of slum at $t=15$. New slum dwellers migrate to the previous slums for new opportunities

income group, these spaces may be re-occupied by lower income households who provide second order services to the higher income households leading to the formation of a slum. Therefore, we see that the oscillatory behaviour or migration of slums near to residential construction site is a unique process and must be included in the model. Further, we observe that during the initial years, the households in Hoodi were primarily single or double person household representing migrant labours. Later, however we see a shift in the household structure, with more families 
getting settled and working as helpers, cleaners or hawkers, servicing the higher income group.

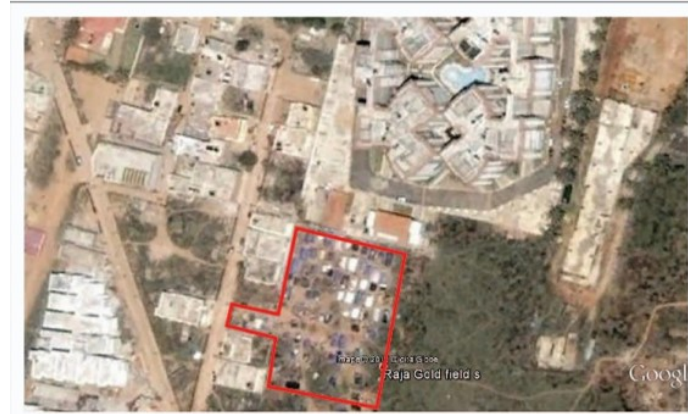

A) 2008

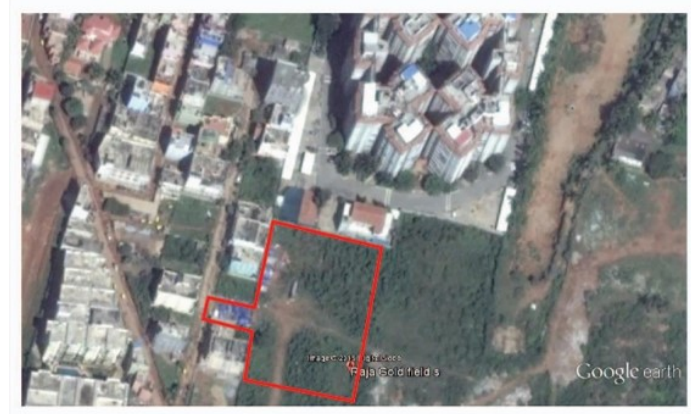

C) 2013

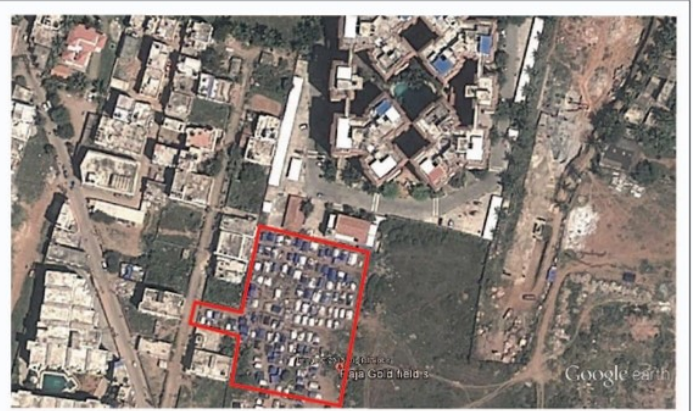

B) 2012

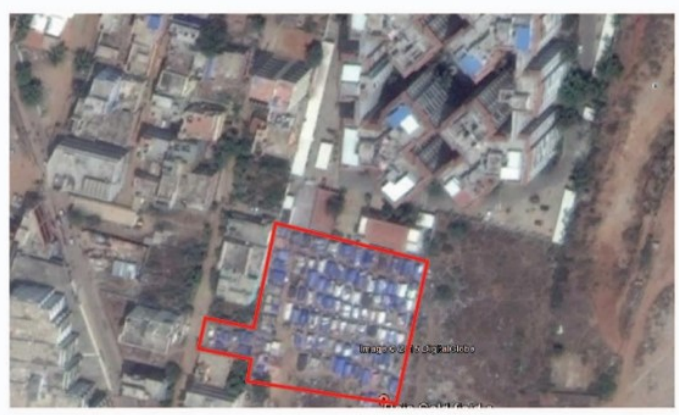

D) 2015

Figure 7.29: Emergence and Growth of a slum in Hoodi, Bangalore (marked with a red polygon). a) Slums emerge near a construction Site in 2008. b) Slum grows near the same site. c) Slum disappear when construction is complete in 2013. d) A slum re-emerge at the same site in 2014 (IMAGE SOURCE: Google Earth).

\subsection{Discussion}

In this chapter we have demonstrated that social and economic satisfaction may have a strong impact on the residential choices of slum dwellers in Bangalore. In order to quantify the importance of model parameter $\omega$ on simulation output and to characterize their effects, we perform sensitivity analysis as described in section 7.3.4. While we performed many different SA's, we report here three results that illustrate the important social and economic dynamics of the model. Significantly, in the context of a shrinking population the presence of social satisfaction leads to a slower rate of decline, until a critical population size is reached, after which the population declines rapidly. Therefore, initially a high social satisfaction applies a 


\section{RESIDENTIAL CHOICE MODEL}

stabilizing effect, which means that despite more attractive economic opportunities, the social satisfaction the agents derive from living in the neighbourhood of social contacts is a strong motive to stay. However, given that a lack of economic opportunities causes a decline in the population, the social satisfaction will decrease as a function of the number of social contacts that move. Therefore, as more agents move out of a slum, the social satisfaction of the remaining agents in its network will decrease, accelerating the emigration of agents from the slum. We also find that higher social satisfaction in a slum could lead to a sharper transition between the slow and fast population decrease stages will be. We show that commute distance is critical for slum households, and induces a trade-off between distance to work and the choice of slums. The critical population and commute distance is interesting for an urban planner in formulating slum management strategies as it enables them to make more fine-grained projections of household types that are more likely to move away, and for what reasons. Further, the model allows for exploring how creating jobs in different occupational categories across different social groups may impact the residential choices of slum dwellers.

\subsubsection{Limitations of the Model}

One of the key challenge is the parametrisation of the economic and environmental satisfaction. In the current study we used the per capita rental as a proxy for environmental quality, as this is capable to capture rather different environmental preferences of people, but we are aware that more elaborate ways exist to measure environmental quality. However, the essence is that the populations experience of environmental quality and satisfaction is being measured, so for the time being relative simple proxies may be sufficient.

For a more accurate empirical implementation of the model, it is desirable to collect a representative growth plan for the encompassing city and land use data. Given sufficient data are available the model can be applied to study the population dynamics of municipalities in a detailed level. Moreover, the model might give indications if a homogeneous or heterogeneous development is preferred for a region as a whole. In that sense we hope that the model we have developed will contribute to the development of efficient economic strategies for local slum communities. One of the other limitation of the model is that it does not simulate a two-worker slum household and the relation between employment dynamics of partners and residential choice. 


\section{Chapter 8}

\section{Conclusion and Future Work}

The Millennium Development Goal (MDG) report 2015 highlights the urgent need to determine the root causes of urban poverty and growth of slums in urban centres of developing countries. The key agenda of post-2015 MDG is to integrate economic, social and environmental dimension of sustainable development into policy. Reflecting on the MDGs, this dissertation aims to understand the dynamic of slum dwellers in developing countries. According to the UNhabitat, around $33 \%$ of the urban population in developing countries lived in slums. Slums emerge and grow due to a combination of demographic, social, economic and political reasons. Rural-urban migration, urbanization, poor housing planning, colonialism and segregation and social conflicts are often attributed to the growth of slums. This dissertation presents a novel interdisciplinary approach integrating ABM, GIS and DCM into a single computational model, namely Dynaslum, to explore social and economic dynamics of slum dwellers in a spatio - temporal environment. Dynaslum is implemented based of the data from Indian city of Bangalore to exemplify its role in policy making in the real world. Specifically, we use multivariate statistical methods on a novel on-field survey dataset from 37 slums in Bangalore to derive rules for the agents in Dynaslum. Dynaslum includes several growth factors (see table 8.1) and provides an exploratory tool to investigate slums as complex non-linear dynamic systems by incorporating the key processes that were missing in the previous models, thus closing the gap between policy and modelling. This chapter further summarizes the findings and provide concluding remarks (section 8.1) followed by the policy implication of the findings (section 8.2). Finally, in section 8.3 this dissertation concludes with a discussion on future work and the way forward. 


\begin{tabular}{|c|c|c|}
\hline Growth Factors & Dynaslum & Comment \\
\hline$\rho_{P}$ & Yes & $\begin{array}{l}\text { Dynaslum improves on existing population dynamics by incorporating } \\
\text { the effect of religion, caste, language and occupation. The household } \\
\text { structure in Dynaslum is an emergent phenomenon driven by various } \\
\text { socio-demographic factors discussed in chapter } 6 \text { and } 7 \text {. In particular, } \\
\text { Dynaslum also incorporates gender-preference at birth. }\end{array}$ \\
\hline$\rho_{E}$ & Yes & $\begin{array}{l}\text { The economic growth of the city is not explicitly modelled. However, } \\
\text { economic growth impacts home leaving, migration rate and the informal } \\
\text { economy which is modelled in Dynaslum. }\end{array}$ \\
\hline$\rho_{I}$ & Yes & $\begin{array}{l}\text { The informal economy is included in Dynaslum. This is explained in } \\
\text { chapter } 7 \text {, where each agent is assigned a job. The model allows for } \\
\text { simulating the economic mobility of the agents based on their skill and } \\
\text { the expected utility. }\end{array}$ \\
\hline$\rho_{G}$ & No & The role of government is not included in Dynaslum. \\
\hline$\overline{\rho_{H}}$ & Yes & $\begin{array}{l}\text { The household dynamics is included in Dynaslum. This is explained in } \\
\text { chapter } 7 \text {, where each agent tries to optimise the per capita rent-income } \\
\text { ratio at different stages of their life cycle. Each household at every time } \\
\text { step tries to select an activity (change in job, change in slum) based on } \\
\text { their current satisfaction level. }\end{array}$ \\
\hline$\rho_{T}$ & No & Slum typology is not implemented in Dynaslum. \\
\hline$\rho_{S}$ & No & Street patterns are not implemented in Dynaslum. \\
\hline
\end{tabular}

Table 8.1: Growth factors included in Dynaslum. $\rho_{P}$ - Population Dynamics, $\rho_{E}$ - Economic Growth, $\rho_{I}$ - Informal Economy, $\rho_{G}$ - Politics Of Slum, $\rho_{H}$ - Housing Dynamics, $\rho_{T}$ - Local Topography, $\rho_{S}$ - Street Pattern 


\subsection{Summary of Findings}

In this section we highlight the key findings of this dissertation. The key research question that guided the design and implementation of Dynaslum is listed in chapter 1. The research questions also necessitated a multi-disciplinary review of existing slum literature. First, previous studies from social sciences and ecology were reviewed to identify the growth factors of slums. An ex-post analysis of key slum policies revealed key processes that must be included in the model. Second, several computation models of varying complexity have been developed over the years to simulate the growth and emergence of slums. In order to review these models, an evaluation framework was developed in chapter 3. Agent based models, with their approach to simulate individual agents and their interactions, were found to be most suitable to simulate the complex process of slum dynamics. We also found that existing models did not attempt to simulate population dynamics of slum households or the various awakening process that trigger residential mobility of slum dwellers. In the few cases, that this was attempted, it was observed that the models were too simple to analyse the effects of micro behaviour of slum dwellers on the growth and emergence of slums. In other words, the models did not generate any emergent phenomenon and were limited to data fitting. As a first step to modelling, demographic and socio economic data from Bangalore were analysed using NFHS 2, 3 and census surveys. Further, to model residential choice and inter-slum migration data was collected from 37 slums in Bangalore (explained in chapter 4). The data analysis further reaffirmed the importance of capturing population dynamics in the growth and emergence of slums. The household size and composition and its socio-economic conditions plays a role in the choice of slums. For e.g. a child rearing family may prefer a slum close to an education facility.

Dynaslum advances the understanding of underlying processes in slum formation. It contributes to the growing literature of simulation models to study the emergence of slums at spatio-temporal scales. The approach to model growth and emergence of slum is novel and unique as it explicitly includes household composition and structure, social groups like origin, religion and economic situation of the actors in the decision making process. Dynaslum departs from previous simulation models on slum in two important dimension. First, all of the previous models discussed in chapter 3, models slums dwellers as a homogeneous economic agent. Few models categorise them as high, low and medium income group, which leads to loss of information in modelling their behaviour. In chapter 4, we have shown that slum dweller are not 


\section{CONCLUSION AND FUTURE WORK}

merely economic agents, they have an important social dimension which needs to be incorporated in the model. We also show in the review of slum policies and in chapter 2 , that slums in Bangalore are extremely heterogeneous and their behaviour differs based on their religion, language, household life cycle and earning potential. Dynaslum models an individual as a social, biological and economic agent and incorporates the heterogeneity in the model. Second, most previous models adopt an iterative approach to model the choice of slums and do not include economic and residential mobility.

In chapter 5 we quantitatively prove Tilly's theory of group segregation by applying multivariate statistical techniques on the data from 37 slums in Bangalore. We find that slums in Bangalore have high degree of segregation based on religion and language. This segregation leads to strong in-group connections which in turn produces "opportunity hoarding" among closed social groups. While opportunity hoarding does not necessarily result in societal exclusion, it may potential lead to categorical inequality. It can couple with exploitation to create damaging differentials in opportunities and rewards among groups in society. In case of slums in Bangalore, data shows that family-run stores, and other types of small-scale enterprise often operate with little or no exploited labour, but nevertheless gain from the 'rents' provided by exclusive use of a site, stock, and costumers. Concentration of blue collared public sector jobs among Hindus indicates that they organise themselves to secure a monopoly over jobs, often supported by the state. Further, we find that formation of such social groups also leads to differentiated behaviour. For e.g. we find that there is a difference in the fertility behaviour of Muslims are very different from Hindus. Muslims have a strong preference of male child which is responsible for their higher fertility rates. This reduces the effectiveness of schemes like "bhagyalakshmi" which promote female child birth. Similarly, we found that most Muslim dominated slums are located at the periphery of the city with less access to the welfare system. These findings demonstrate that slum typologies should not only focus on physical attributes of slums (as mandated by RAY) but it should also focus on socio-economic condition of slum dwellers. The findings clearly highlight that policy interventions needs to consider these highly segregated groups and heterogeneous behaviour to increase the success rate as there may not be a one-size fits all solution.

An important benefit of agent based modelling is the prospect to model the micro behaviour of each individual, which makes it an effective method to investigate population and residential choice dynamics of slum dwellers. Simulating the individual slum dwellers allows emerging behaviour to be analysed. The age and household structure of a population is influenced by 
several factors such as access to medical facilities, population control strategies, evolving societal customs. However, these factors influence different sections of the society in a different manner. As such it is important to understand the difference in household dynamics between different sections of the society. In chapter 6 we have presented an ABM capable to simulate the household dynamics of slums in developing countries by incorporating language and religion. Based on our key research objectives, the population dynamics model demonstrates a fine balance between minimalism and practicality. The design calibration and validation of Dynaslum is achieved using data from NFHS survey, census of India and unique novel on-field survey from 37 slums in Bangalore.

In its current form, the model can reproduce convincing distribution of household structure, emerging from the interactions of various social and demographic events. Further, the model generates useful data on family relationships among agents that may be used to create multilevel extended family networks. However, in chapter 6 , we have focused on understanding the difference in household life cycle (HLC) of slums and non-slum households in the city of Bangalore. We find that slum households exhibit a large variation in their HLC as compared to non-slum households. We have used the model to determine the key factors that drive this difference. Through the model we find that higher rate of home leaving among young adults due to adolescence and marriage are the key drivers of the difference in HLC. We show that home leaving also leads to large number of smaller households and ineffective resource consumption per capita. The large variation observed in the HLC of slum households also makes it difficult for urban planners to provide appropriate low cost housing for slum dwellers. In past, it has been observed that most resettlement colonies were either underutilized or too small for a slum household. As a result, the households rent these low cost housing to higher income groups and move out to other slums. Though, this phenomenon is documented in social science literature, it is for the first time we can prove it using a computational model. The findings may have policy implications, as planners can now focus on strategies to reduce the variation in HLC among slum dwellers. This in turn will not only reduce the formation of new smaller slum households but also increase the acceptance of resettlement colonies as policy makers would be able to plan for a fixed family size.

In chapter 7 we have described the residential choice model which integrates economic, social and environmental satisfaction in the decision making of slum dwellers. Previous residential choice models lack a robust theoretical basis and are not suitable to simulate slum dynamics as they are a based on a formal market clearance methodology. The agent based residential choice 


\section{CONCLUSION AND FUTURE WORK}

model apply discrete choice experiments (DCE) to derive behaviour rules of the agents. The model is a step forward from previous attempts as it not only captures residential mobility but also includes the effect of social and economic mobility. Further, the model is able to simulate the "awakening process" of slum dwellers that leads to residential and economic mobility. The discrete choice experiments indicates that social factors (homophily) plays a crucial role in choice of slums. Slum dwellers prefer to live close to family and similar social groups (religion, language). We also find that slum households with income at the top and bottom $10 \%$ have a preference for size or quality of housing. While slum households with top 10\% income prefer larger houses the bottom 10\% usually live in the smallest dwellings. We have incorporated these features in the model and implement heterogeneity of agents by assigning individual weights to the social, economic and environmental satisfaction function which are updated at each time step based on the situation of individual agents. Further, we present a sensitivity analysis and validation of inter slum migration based on data from 37 slums in Bangalore. The model demonstrates that the outcome of residential choice dynamics of slum dwellers is not equal to the sum of individual structure. The fact that individuals influence each other makes the outcome of the group process different from the outcome of individual decision making in isolation.

The residential choice model produces three key findings. First, in the context of a depopulating slum, higher social satisfaction leads to a slower rate of decline, until a critical population size is reached, after which the population declines rapidly. Therefore, initially a high social satisfaction applies a stabilizing effect, which means that despite more attractive economic opportunities, the social satisfaction the agents derive from living in the neighbourhood of social contacts is a strong motive to stay. However, given that a lack of economic opportunities causes a decline in the population, the social satisfaction will decrease as a function of the number of social contacts that move. Therefore, as more agents move out of a slum, the social satisfaction of the remaining agents in its social network will decrease, accelerating the emigration of agents from the slum. Significantly, we also find that higher social satisfaction in a slum could lead to a sharper transition between the slow and fast population decrease. Second, we find that there is a critical value of commute distance (around 8-10 kilometres) for slum households in Bangalore, which induces a trade-off between distance to work and the choice of slums. Finally, the model shows that smaller and temporary slums usually split and migrate to different parts of the city depending on economic opportunities available in other parts of the city. The above findings are verified using real data from Bangalore and hence show that Dynaslum could be 
used to predict when, where and how slums grow and emerge in Bangalore. In the next section we discuss the possible policy implications of the above findings in the context of slum management in developing countries.

\subsection{Policy Implication}

The primary outcome of this dissertation are the key findings of Dynaslum described in the previous section, that could be used by urban planners to evaluate the consequences of several prospective slum management policies ex-ante. It is hoped that the computational model developed for Bangalore, India, could be customized and applied in other cities within India and potentially in other developing countries to formulate effective slum management strategies in future. In this section we discuss the policy implications in context of Bangalore, India.

The multivariate statistical analysis in chapter 5 shows that social, cultural and economic attributes of slum dwellers has a massive influence on growth and emergence of slums. It is evident that economic growth and urbanization alone cannot reduce poverty in cities of developing world as the it is not shared equally among all sections of the society. Therefore, it is important to understand the social segregation and opportunity bias among slum dwellers.

In past the size of resettlement dwellings have been designed based on the duration of the dwellers in the slum. We find that this is counter productive and the size of the dwelling should be based on the family size. In chapter 6 , we find that the household life cycle of slum shows a large variation as compared to non-slum urban household largely driver by home-leaving and couple formation. In such circumstances, designing low-cost housing is difficult as they may be under-utilized or over-utilized. Data from slums also indicate the cost of squatting is very less compared to build a new house in a formal real estate market. This may be one of the reasons why the rate of home loaning is higher in slums which leads to the formation of more households and slum sprawl. Policies have been implemented in UK and Singapore in the form of "bedroom tax" and "3G housing" to constrain home leaving among youth. Dynaslum suggests a paradigm shift from population control to household control to stem the growth and emergence of slums.

The residential choice model adds an important dimension to Dynaslum, which allows it to be used as an decision support system for policy makers as they can now analyse the effect of economic, social and environmental individual satisfaction on the growth and emergence of slums. For e.g. as rentals increase in the slums at the core of the city, the elderlies and the 
low income slum households may migrate towards the periphery of the city. The findings of Dynaslum indicates that the social satisfaction, job opportunities and commute distance plays an important role in the residential mobility of slum dwellers. Therefore, policy makers can now use the above findings to implement resettlement colonies a-priori and thus prevent formation of new slums. Similarly, other interventions such as health facilities and schools can be planned based on the information provided by the model.

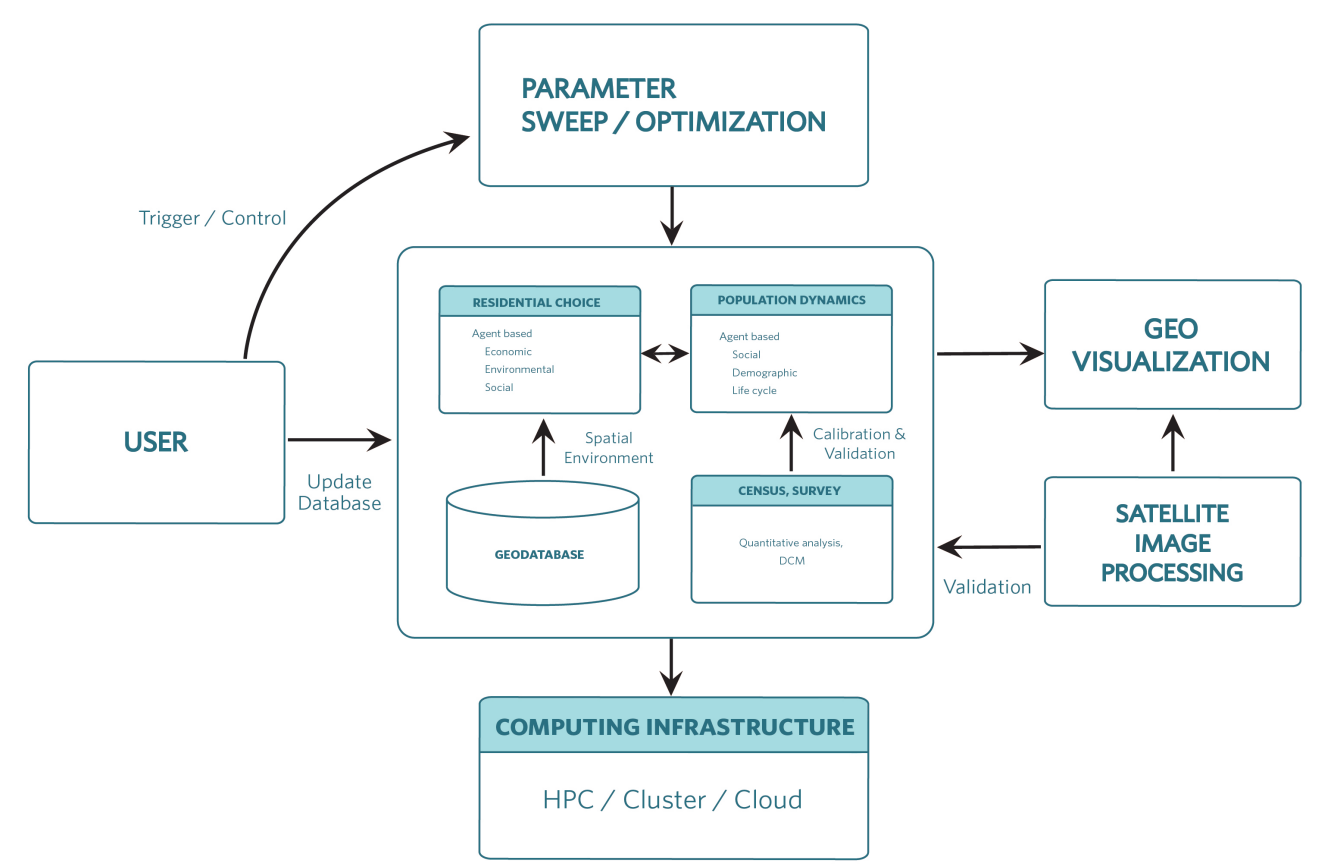

Figure 8.1: Schematic Diagram Dynaslum

\subsection{Future Extension}

The key objective of Dynaslum was to build a high-resolution agent-based models that can describe the growth dynamics of slums in Bangalore. Such a model will create a virtual slum that decision-makers, and researchers, can use to explore how different policies would influence the growth, development or contraction of slums. The team plan to build on their existing work and develop the model as a decision support system for slums in general, which will help guide experts when evaluating or designing policies to improve conditions within slums. 
This involves developing new computational methods for analysing satellite images and new data visualization techniques for simulation steering. Through extending the existing decision support prototype, and applying it to a new domain of slum policy, the hope is to generalize the current software. Therefore, this proposal will aim to develop a reusable software framework for decision support and disseminate it to other users.

The team plans to build on the existing models discussed in this dissertation and datasets with the aim to develop four new aspects to the research as shown in figure 8.1. Firstly, the existing model will be developed further to include spatial information (enabling the model to indicate areas likely to show emergence of new slums) based on the master city plan or development plan of Bangalore. Secondly, we plan to develop methods for processing satellite images to more accurately chart the growth of slums over the last few decades. Thirdly, we plan to including accessibility measures into the model by integrating with MATSIM model. Therefore, various public transport projects such as Bangalore Metro Rail Corporation (BMRC) and Bangalore Metropolitan Transport Corporation (BMTC) of Bangalore could be incorporated as input parameters for each housing site in the model. Finally, we plan to integrate the validated model into a decision support front-end that will allow policy makers to experiment and understand the possible outcomes of their policy decisions 
8. CONCLUSION AND FUTURE WORK 


\section{Appendix A}

\section{Classifications in the Survey Data}

During the survey, the respondents were asked their occupations, and this was recorded without coding. These occupations were then categorised during data clean-up. The categorisation underwent 7 iterations and the final version is presented in table A.1.

\begin{tabular}{|l|l|}
\hline Occupation ID & Occupation Category \\
\hline 1 & Professional - White Collar \\
2 & Professional - Blue Collar \\
3 & Sales and Small enterprises \\
4 & Self employed - Sales \\
5 & Self employed - Repair and service \\
6 & Home based employment \\
7 & Domestic and cleaning services \\
8 & Manufacture and Small Industry \\
9 & Security \\
10 & Transport Services \\
11 & Construction \\
12 & Manual Labour \\
13 & Unclassified \\
\hline
\end{tabular}

Table A.1: Classification of jobs into Occupation Category

Out of the data of the 5634 people in the survey, 2049 people reported their income. The income was further categorised into 9 levels as shown in table A.2. Household income is calculated by calculating the sum of the individual incomes in the household. Similar to individual incomes, the household income distribution resembled a long-tail distribution (Fig 4.6). The income categorisation had to be chosen which best represented this distribution. 


\begin{tabular}{|l|l|}
\hline Income Category & Income (INR) \\
\hline 1 & $200-1300$ \\
2 & $1300-2400$ \\
3 & $2400-3500$ \\
4 & $3500-4600$ \\
5 & $4600-5700$ \\
6 & $5700-8000$ \\
7 & $8000-12000$ \\
8 & $12000-18000$ \\
9 & Greater than 18000 \\
\hline
\end{tabular}

Table A.2: Classification of Income into Income Category

- Clustering to identify possible interval sets: k-means clustering was used on the income vectors to identify clusters and intervals. This clustering algorithm was run three times to identify $3,4,5,6,7,8,9$ and 10 clusters, and their respective intervals. This was performed to identify the number of intervals we would require to represent the income distribution.

- Identifying interval sets which best represent a long-tail distribution: From the results of the clustering, the following interval sets were identified as best representing a long-tail distribution:

Age of the slums were required to understand its relationship with the facilities and infrastructure available within these slums, and to understand how slum demographics vary based on whether the slums are rehabilitated, non-rehabilitated, declared, old or new. The age of the slums was acquired through a personal interview with Mr. Issac Arul Selva of Slum Jagatthu organization, previous slum studies and Karnataka Slum Clearance Board surveys of 1994, 2008 and 2010. This data contains information on when the slum came into existence and when the slums got rehabilitated. The age of the slums was corroborated based on these multiple versions, and a final version is presented in table A.3. 


\begin{tabular}{|c|c|c|c|}
\hline Slum ID & Slum Name & Slum Location & Slum Age \\
\hline 1 & Ambedkar Nagar Okalipu & Core & 40 \\
\hline 2 & Ambedkar Nagar Koraman & Core & 43 \\
\hline 3 & Vijinapura & Periphery & 30 \\
\hline 4 & Nellorepuram & Periphery & 30 \\
\hline 5 & Haavadigara Colony & Periphery & 20 \\
\hline 6 & Nagappagarden & Core & 70 \\
\hline 7 & Thubarahalli & Periphery & 10 \\
\hline 8 & VST Colony & Core & 28 \\
\hline 9 & Christian Colony & Core & 50 \\
\hline 10 & Tank Mohalla & Core & 40 \\
\hline 11 & Manjunath Nagara & Periphery & 20 \\
\hline 12 & Yaarab Nagara & Intermediate & 70 \\
\hline 13 & Sweepers colony & Periphery & 70 \\
\hline 14 & Kaveri Nagar & Periphery & 20 \\
\hline 15 & Gorguntepalya & Periphery & 20 \\
\hline 16 & Buddhanagar & Periphery & 40 \\
\hline 17 & Sadaramangala & Periphery & 20 \\
\hline 18 & Maya Bazaar & Core & 80 \\
\hline 19 & Swatantrapura & Periphery & 20 \\
\hline 20 & Alemaari & Periphery & 10 \\
\hline 21 & Ashwath Katte & Periphery & 15 \\
\hline 22 & Corporation Colony & Intermediate & 45 \\
\hline 23 & Raagigudda & Intermediate & 40 \\
\hline 24 & Swagath & Core & 50 \\
\hline 25 & Singapura & Periphery & 65 \\
\hline 26 & Thigalara Palya & Periphery & 35 \\
\hline 27 & Roshan Nagara & Core & 20 \\
\hline 28 & Kuntigrama & Periphery & 15 \\
\hline 29 & Gangondanahalli & Periphery & 35 \\
\hline 30 & Vinobha Nagar & Core & 60 \\
\hline 31 & Siddapura & Core & 60 \\
\hline 32 & Arasu Colony & Intermediate & 25 \\
\hline 33 & Jolly Mohalla & Core & 50 \\
\hline 34 & LR nagar & Core & 60 \\
\hline 35 & Gajendranagar & Core & 50 \\
\hline 36 & MS building & Core & 60 \\
\hline 37 & Hakki Pikki Colony & Periphery & 10 \\
\hline
\end{tabular}

Table A.3: List of slums surveyed 
A. CLASSIFICATIONS IN THE SURVEY DATA 


\section{Appendix B}

\section{Infrastructure in the Slums of Bangalore}

The objective of this section is to look into the present conditions and status of slum households in Bangalore, India. The primary reason for looking at this particular question is to determine whether the slum households, who normally form the poor strata of the urban population, are homogeneous? For greater comprehension, this section attempts to discover the different physical attributes of slum households in Bangalore.

\begin{tabular}{|l|l|}
\hline Water Source & Percentage \\
\hline 1. Individial water conne & 11.9787 \\
2. Makeshift water connec & 0.3549 \\
3. Individual sub connect & 1.5972 \\
4. Mini water supply & 12.7773 \\
5. Public tap & 55.3682 \\
6. Community well/handpum & 2.6619 \\
7. Water tanker & 4.9689 \\
8. Surface water & 0.7098 \\
9. Other vendors & 9.583 \\
\hline
\end{tabular}

Table B.1: Sources of water supply in the Slums of Bangalore

In the context of JNNURM and various other funded programs, Bangalore city has invested in infrastructure and slum upgradation. Government of Karnataka appointed BBMP as the key agency for this coordinated efforts in compliance with the implementation of 74th constitutional amendment which envisages urban decentralization and empowers local bodies. However, the survey data indicates that around $55 \%$ of the slum households depend on public taps for access 


\begin{tabular}{|l|l|}
\hline Electricity Source & Percentage \\
\hline 1. Metered connection & 76.087 \\
2. Unmetered connection & 12.2873 \\
3. Unofficial or makeshift connection & 3.6862 \\
4. Through sub-contractor on flat rate basis & 0.3781 \\
5. No electricity available & 7.5614 \\
\hline
\end{tabular}

Table B.2: Sources of electricity in the Slums of Bangalore

to water. On average an individual household spends around 2 hours per day to fetch water for the household. In contrast, around $71 \%$ households have access to electricity through metered connection in comparison to $37 \%$ in rural Karnataka. Around 5\% households do not have toilets

\begin{tabular}{|l|l|}
\hline Toilet Type & Percentage \\
\hline 1. No toilet & 5.2342 \\
2. Toilet shared with neighbours & 32.0937 \\
3. Community toilet - free & 9.3664 \\
4. Open space & 1.5152 \\
5. Common toilet - paid & 12.3967 \\
7. Other & 18.4573 \\
\hline
\end{tabular}

Table B.3: Toilet facilities in the Slums of Bangalore

and $54 \%$ households share toilets with neighbours or use public toilets. Most slum households have their own kitchen (68\%) but around $50 \%$ use kerosene as fuel, $24 \%$ households use firewood and only $23 \%$ use LPG as cooking fuel.

\begin{tabular}{|l|l|}
\hline Kitchen Type & Percentage \\
\hline 1. Within house-seperate kitchen & 68.7327 \\
2. Within the house- as a part of another room & 28.7697 \\
3. Outside the house & 1.8501 \\
4. Kitchen shared with other households & 0.185 \\
5. No cooking & 0.185 \\
6. Other & 0.2775 \\
\hline
\end{tabular}

Table B.4: Kitchen types in the Slums of Bangalore

The construction material varies depending on the structure of the house. Most households have metal roofs built on walls with mud and flooring is usually brick based.

"The CEGA researchers note that inadequate housing poses serious health risks around the 


\begin{tabular}{|l|l|}
\hline Bathroom Type & Percentage \\
\hline Own & 84.1182 \\
Shared & 15.88 \\
\hline
\end{tabular}

Table B.5: Bathroom types in the Slums of Bangalore

\begin{tabular}{|l|l|l|l|}
\hline Roof Material & Percentage & Wall Material & Percentage \\
\hline 1. Thatch & 3.156 & 1. Thatch & 1.1618 \\
2. Plastic & 2.1641 & 2. Plastic & 2.2341 \\
3. Tiles & 3.3363 & 3. Mud & 34.9419 \\
4. Slate & 0.0902 & 4. Brick & 0.1787 \\
5. Metal/Asbestos & 66.6366 & 5. Wood & 12.1537 \\
6. Brick & 0.1803 & 6. Metal/Asbestos & 9.2046 \\
7. Stone & 0.0902 & 7. Burnt Brick & 0.3575 \\
8. Concrete & 23.6249 & 8. Stone & 3.4853 \\
9. Other & 0.7214 & 9. Concrete & 36.1037 \\
\hline
\end{tabular}

Table B.6: Roof and Wall materials used in the Slums of Bangalore

globe for 600 million urban residents, almost half of them living in slums. These problems also contribute to the deaths of 3 million children every year from parasitic infections associated with diarrhoea, malnutrition and micro nutrient deficiencies such as anaemia, a widespread problem that slows cognitive development".

\begin{tabular}{|l|l|}
\hline Floor Material & Percentage \\
\hline 1. Mud & 6.9307 \\
2. Wood or Bamboo & 1.8002 \\
3. Brick & 84.2484 \\
4. Stone & 6.7507 \\
5. Cement & 0.27 \\
\hline
\end{tabular}

Table B.7: Floor materials used in the Slums of Bangalore 
B. INFRASTRUCTURE IN THE SLUMS OF BANGALORE 


\section{Appendix C}

\section{Residential Choice Component}

In this section we describe the state variables of the different entities in the Residential Choice Model. The table C.1 shows the state variables of an individual. Different individuals combine to form a household, the state variables shown in table C.2. Jobs are randomly created from the distributed; table C.3 shows the various state variables for a job. Finally, table C.4shows the state variables for a slum.

In this section we also present the algorithm 1, for updating the weights of social, economic and environmental satisfaction for each household at every time step.

\begin{tabular}{|l|l|}
\hline \multicolumn{1}{|c|}{ Variable } & \multicolumn{1}{c|}{ Explanation } \\
\hline Household ID & Unique ID to identify a household \\
\hline Person ID & Unique ID to identify an individual in a household \\
\hline Status & Worker, Student, Retired, Unemployed, Inactive \\
\hline Age & Age of the Head of Household \\
\hline Job ID & If worker, the job ID \\
\hline Income & If worker, income \\
\hline Skill ID & One of the occupation ID based on distribution \\
\hline
\end{tabular}

Table C.1: State Variable for a Individual 


\begin{tabular}{|c|c|}
\hline Variable & Explanation \\
\hline Household ID & Unique ID to identify a household \\
\hline Slum ID & Unique ID to identify an individual in a household \\
\hline Rent & Actual Rent or HO (Home owner) or HL (Home Less) \\
\hline Religion & $\begin{array}{l}\text { Religion with the following possible values: } \\
\text { 0:Hindu. } \\
\text { 1:Muslim. } \\
\text { 2:Christian. } \\
\text { 3:Others }\end{array}$ \\
\hline Language & $\begin{array}{l}\text { Language with the following possible values: } \\
\text { 0:Kanada } \\
\text { 1:Tamil } \\
\text { 2:Telegu } \\
\text { 3:Hindi } \\
\text { 4:Urdu: } \\
\text { 5:Others }\end{array}$ \\
\hline Type & $\begin{array}{l}\text { A household type, with the following possible values: } \\
\text { 0: single. } \\
\text { 1: mono-parental family. } \\
\text { 2: couple without children. } \\
\text { 3: couple with children. } \\
\text { 4: others }\end{array}$ \\
\hline
\end{tabular}

Table C.2: Explanation of State Variable for a Slum Household

\begin{tabular}{|l|l|}
\hline \multicolumn{1}{|c|}{ Variable } & \multicolumn{1}{c|}{ Explanation } \\
\hline Job ID & Unique Job identification \\
\hline Location & Location where the Job is available \\
\hline Occupation Category & Unique Job identification \\
\hline Vaccancy & Number of vaccancies \\
\hline Expiry & Number of timestep the job is active \\
\hline Salary & The salary for the job ID \\
\hline
\end{tabular}

Table C.3: State Variable of Jobs 


\begin{tabular}{|l|l|}
\hline \multicolumn{1}{|c|}{ Variable } & \multicolumn{1}{c|}{ Explanation } \\
\hline Slum ID & Unique identity of each slum \\
\hline Capacity & Maximum number of households \\
\hline Location & Latitude and Longitude \\
\hline Rent Distribution & Core, Intermediate, Periphery \\
\hline Slum Age & Age of the slum \\
\hline
\end{tabular}

Table C.4: State variable for a Slum

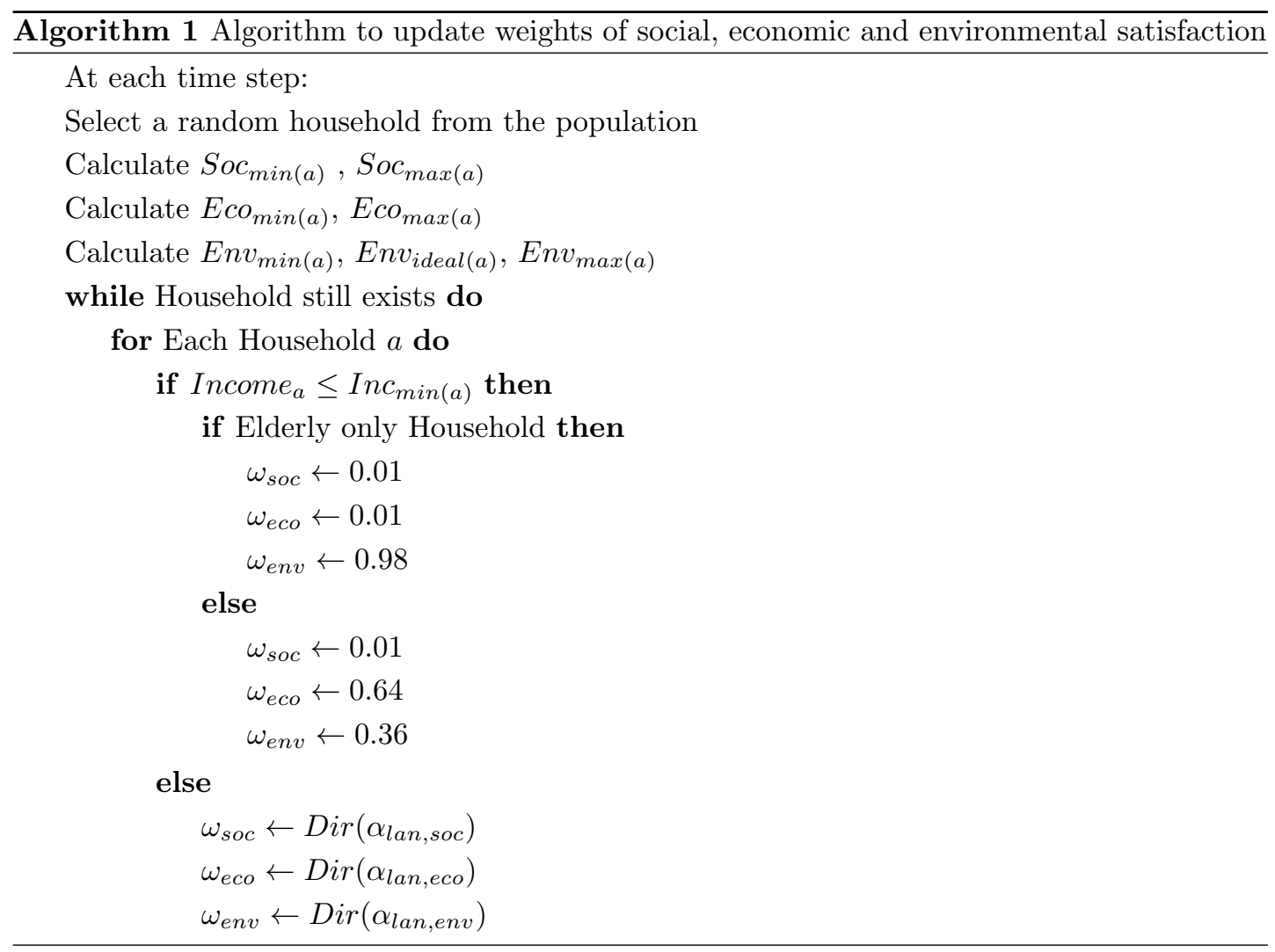


C. RESIDENTIAL CHOICE COMPONENT 


\section{Bibliography}

[1] Censusofindia. Data products available from The Registrar General and Census Commissioner, India, New Delhi, Ministry of Home Affairs, Government of India. [Online]. Accessed on December 12, 2013.

[2] IIPS. National family health survey (NFHS-3):2005-06. Technical Report 06, International Institute for Population Sciences (IIPS) and Macro International, 2007.

[3] IIPS. National family health survey (NFHS-2): 1998-99. Technical report, International Institute for Population Sciences (IIPS) and ORC Macro, 2000.

[4] Debraj Roy, Michael Harold lees, Bharath Palavalli, Karin Pfeffer, and MA Peter Sloot. The emergence of slums: A contemporary view on simulation models. Environmental Modelling \& Software, 59:76-90, 2014.

[5] UN-HABITAT. For A Better Urban Future: A mission for the 21st century. United Nations Human Settlements Programme, Nairobi, Kenya, 2011.

[6] Amit Patel, Andrew Crooks, and Naoru Kolzumi. Slumulation: An AgentBased Modeling Approach to Slum Formations. Journal of Artificial Societies and Social Simulation, 15(4):2, 2012.

[7] C. Richter, G. Miscione, R. De', and K. Pfeffer. Enlisting SDI for urban planning in India: local practices in the case of slum declaration. In Z. NEDOVIĆ Budić, J. Crompvoets, and Y. Georgiadou, editors, Spatial data infrastructures in context: north and south, pages 157-179, Boca Raton, FL, 2011. CRC Press.

[8] UNDP. Resolution adopted by the General Assembly : United Nations Millennium Declaration. [Online]. Accessed on December 12, 2013, 2000. 
[9] Barjor E. Mehta, Arish Dastur, and S. Janus, editors. Approaches to Urban Slums. World Bank Publications, Washington D.C., US, February 2008.

[10] UN-HABITAT. Participatory Slum Upgrading And Prevention : Millennium City Of Accra, Ghana. Technical report, Participatory Slum Upgrading Program, UN-HABITAT, Accra, Ghana, 2011.

[11] Амiтaвн Kundu. Making Indian Cities Slum-Free. Economic and Political Weekly, 48(17):15-18, Apr 2013.

[12] Vichai Viratkapan and Ranjith Perera. Slum relocation projects in Bangkok: what has contributed to their success or failure? Habitat International, 30(1):157 $-174,2006$.

[13] Akie Takeuchi, Maureen Cropper, and Antonio Bento. Measuring the welfare effects of slum improvement programs: The case of Mumbai. Journal of Urban Economics, 64(1):65 - 84, 2008.

[14] GovernmentOfIndia. Rajiv Awas Yojana Guidelines for Slum-free City Planning. Government of India, Ministry of Housing and Urban Poverty Alleviation, 2010.

[15] P. BALl. Why Society is a Complex Matter: Meeting Twenty-first Century Challenges with a New Kind of Science. Springer, Berlin, Germany, 2012.

[16] R. E. Wilkes. Household Life-Cycle Stages Transitions, and Product Expenditures. Journal of Consumer Research, 22(1):27-42, 1995.

[17] M. C. Gilly And B. M. Enis. Recycling the family life cycle: A proposal for redefinition. Advances in consumer research, 9(1):271-276, 1982.

[18] WorldBank. Cities Alliance for Cities Without Slums : Action Plan for Moving Slum Upgrading to Scale. World Bank, Washington D.C., US, 2013.

[19] UN-HABITAT. The State of the World's Cities 2006/200\%: The Millennium Development Goals and Urban Sustainability: 30 Years of Shaping the Habitat Agenda. State of the world's cities report. Earthscan, London, UK, 2006.

[20] IIHS. Urban India 2011: Evidence Report. In India Urban Conference: Evidence and Experience, pages 1-83, New Delhi, India, November 2011. Indian Institute for Human Settlements. 
[21] Swaminathan S Anklesaria Aiyar. Slums are hubs of hope, progress and dignity. [Online]. Accessed on December 12, 2013., 2013.

[22] Haris Gazdar. A review of migration issues in Pakistan. In Regional Conference on Migration, Development and Pro-Poor Policy Choices in Asia., pages 1-25, 22-24 June 2003 in Dhaka, Bangladesh, June 2003. Regional Conference on Migration, Development and Pro-Poor Policy Choices in Asia.

[23] Le Van Thanh. Migrants and the socio-economic development of Ho Chi Minh City. In NIE-SEAGA-Conference, pages 1-13, Sustainability and Southeast Asia: Southeast Asian Geography Association, 28-30 November 2006, Singapore, November 2006.

[24] Sandhya Mahapatro. The changing pattern of Internal Migration in India: Issues and Challanges. In European Population Conference, pages 1-18. Stockholm University, Sweden, June 2012.

[25] COHRE. Women, Slums And Urbanisation: Examining the Causes and Consequences. Centre on Housing Rights and Evictions (COHRE), Geneva, Switzerland, May 2008.

[26] Homelessinternational. The Impact of Slums. [Online]. Accessed on December 12, 2013, June 2010.

[27] PlanningCommission. Report Of The Working Group On Urban Poverty , Slums , And Serivce Delivery System. Technical report, Steering Committee on Urbanization Planning Commission, New Delhi, 2011.

[28] Jan NiJman. A study of space in Mumbai's slums. Tijdschrift voor economische en sociale geografie, 101(1):4-17, 2010.

[29] Sabina Rashid. Strategies to Reduce Exclusion among Populations Living in Urban Slum Settlements in Bangladesh. Journal of Health, Population and Nutrition, 27(4):574-586, 2009.

[30] Ben C Arimah. WP/30 The Face of Urban Poverty: Explaining the Prevalence of Slums in Developing Countries. UNU-WIDER Working Paper Series, 2010/30, March 2010. 
[31] Aprodicio A. Laquian. Beyond metropolis : the planning and governance of Asia's mega-urban regions. Woodrow Wilson Center Press. Johns Hopkins University Press, Washington, D.C., US, 2005.

[32] Sanjoy Chakravorty. A New Price Regime. Economic and Political Weekly, 48(17):45-54, Apr 2013.

[33] R. Neuwirth. Shadow Cities: A Billion Squatters, a New Urban World. Routledge, New York, US, 2006.

[34] R. Neuwirth. Stealth of Nations: The Global Rise of the Informal Economy. Anchor Books, New York, US, 2012.

[35] Daniel J. Garr. Expectative land rights, house consolidation and cemetery squatting: Some perspectives from Central Java. World Development, 24(12):1925 $-1933,1996$.

[36] Neha Goel. Squatter Settlements : The Urban Vernacular. In 14th International Planning History Society, pages 1-5, Istanbul, 12-15 July 2010. Istanbul Technical University Faculty of Architecture and Research Center.

[37] Proshun Chakraborty. Parties doing whatever it take to ensure slum votes. [Online]. Accessed on December 13, 2013., February 2012.

[38] H.M. Mathur. Displacement and Resettlement in India: The Human Cost of Development. Routledge Contemporary South Asia Series. Routledge, New York, US, 2013.

[39] Daniel Kaufmann, Aart Kraay, and Pablo Zoido-Lobaton. Aggregating governance indicators. Policy Research Working Paper Series 2195, The World Bank, October 1999.

[40] Richard Harris. A Double Irony: The Originality and Influence of John F. C. Turner. Habitat International, 27:245-269, 2003.

[41] H. De Soto. The Other Path: The Invisible Revolution in the Third World. I.B. Tauris, London, UK, 1989.

[42] H. De Soto. Mystery of Capital: Why Capitalism Triumphs in the West and Fails Everywhere Else. Basic Books, New York, US, 2003. 
[43] Rod Burgess. Petty commodity housing or dweller control? A critique of John Turner's views on housing policy. World Development, 6(9-10):1105-1133, 1978.

[44] Rod Burgess. The Limits of State Self Help Housing Programmes. Development and Change, 16(2):271-312, 1985.

[45] A Gilbert. On the mystery of capital and the myths of Hernando de Soto What difference does legal title make? International Development Planning Review, 24(1):1-19, March 2002.

[46] Peter Abelson. Evaluation of slum improvements: Case study in Visakhapatnam, India. Cities, 13(2):97 - 108, April 1996.

[47] Richard Harris. A crank's fate and the feting of a visionary: reflections on the history of aided self-help housing. Third World Planning Review, 20(3):3-8, August 1998.

[48] V. MukhiJA. Squatters as Developers?: Slum Redevelopment in Mumbai. King's SOAS studies in Development Geography. Ashgate, Aldershot, Hampshire, England, 2003.

[49] E. Liebow. Tally's Corner: A Study of Negro Streetcorner Men. Legacies of social thought. Rowman \& Littlefield, Lanham, MD, 2003.

[50] Somik V. Lall, Mattias K.A. Lundberg, and Zmarak Shalizi. Implications of alternate policies on welfare of slum dwellers: Evidence from Pune, India. Journal of Urban Economics, 63(1):56 - 73, 2008.

[51] Donatien Beguy, Philippe Bocquier, and Eliya Msiyaphazi Zulu. Circular migration patterns and determinants in Nairobi slum settlements. Demographic Research, 23(20):549-586, 2010.

[52] C.N Thomson and P. Hardin. Remote sensing/GIS integration to identify potential low-income housing sites. Cities, 17(2):97-109, 2000.

[53] J. R. Weeks, A. Hill, D. Stow, A. Getis, and D. Fugate. Can we spot a neighborhood from the air? Defining neighborhood structure in Accra, Ghana. GeoJournal, 69(1-2):9-22, 2007. 
[54] I. Baud, N. Sridharan, and K. Pfeffer. Mapping Urban Poverty for Local Governance in an Indian Mega-City: The Case of Delhi. Urban Studies, 45(7):1385-1412, June 2008.

[55] S. O. Mason and C. S. Fraser. Image Sources for Informal Settlement Management. The Photogrammetric Record, 16(92):313-330, 1998.

[56] P. Hofmann, J. Strobl, T. Blaschke, and H. Kux. Detecting informal settlements from QuickBird data in Rio de Janeiro using an object based approach. In Thomas Blaschke, Stefan Lang, and GeoffreyJ. Hay, editors, ObjectBased Image Analysis, Lecture Notes in Geoinformation and Cartography, pages 531-553. Springer Berlin Heidelberg, 2008.

[57] Olena Dubovyk, Richard Sliuzas, and Johannes Flacke. Spatio-temporal modelling of informal settlement development in Sancaktepe district, Istanbul, Turkey. Journal of Photogrammetry and Remote Sensing, 66(2):235 - 246, 2011.

[58] H. Taubenböck and N.J. Kraff. The physical face of slums: a structural comparison of slums in Mumbai, India, based on remotely sensed data. Journal of Housing and the Built Environment, pages 1-24., 2013.

[59] Oleksandr Kit and Matthias Ludeke. Automated detection of slum area change in Hyderabad, India using multitemporal satellite imagery. Journal of Photogrammetry and Remote Sensing, 83(0):130 - 137, 2013.

[60] S Jongpiputvanich, S Veeravongs, and W Wonsekiarttirat. Difficulties in conducting participatory action research to prevent diarrhoea in a slum area of Bangkok. Journal of diarrhoeal diseases research, 16(3):187-93, September 1998.

[61] Monica Rossi. The city and the slum: an action research on a Moroccan and a Roma Xoraxan community in Rome. $\mathrm{PhD}$ thesis, University of Birmingham,UK, December 2010.

[62] R White and G Engelen. Cellular automata as the basis of integrated dynamic regional modelling. Environment and Planning B: Planning and Design, 24(2):235$246,1997$. 
[63] C J Webster and F Wu. Regulation, land-use mix, and urban performance. Part 2: simulation. Environment and Planning A, 31(9):1529-1545, 1999.

[64] Quang Bao Le, Roman Seidl, and Roland W. Scholz. Feedback loops and types of adaptation in the modelling of land use decisions in an agent based simulation. Environmental Modelling and Software, 28(0):83 - 96, 2012.

[65] F. Semboloni. An Urban and Regional Model Based on Cellular Automata. Environment and Planning B: Planning and Design, 24(4):589-612, 1997.

[66] J. Portugali. Self-Organization and the City. Springer Series in Synergetics. Springer, Berlin, Germany, 2000.

[67] David O'Sullivan. Exploring Spatial Process Dynamics Using Irregular Cellular Automaton Models. Geographical Analysis, 33(1):1-18, 2001.

[68] Keith C. Clarke and Leonard J. Gaydos. Loose-coupling a cellular automaton model and GIS: long-term urban growth prediction for San Francisco and Washington/Baltimore. International Journal of Geographical Information Science, 12(7):699-714, 1998.

[69] M. Batтy. Cities and Complexity: Understanding Cities With Cellular Automata, AgentBased Models, and Fractals. The MIT Press, Cambridge, MA, 2007.

[70] K C Clarke, S Hoppen, and L Gaydos. A self-modifying cellular automaton model of historical urbanization in the San Francisco Bay area. Environment and Planning B: Planning and Design, 24(2):247-261, 1997.

[71] I. Benenson and P. Torrens. Geosimulation: Automata-Based Modeling of Urban Phenomena. Wiley InterScience online books. John Wiley \& Sons, London, UK, 2004.

[72] A.J. Heppenstall, A.T. Crooks, and L.M. See. Agent-Based Models of Geographical Systems. Springer Netherlands, Dordrecht, Netherlands, 2012.

[73] G.F. Young and J. Flacke. Agent based model of the growth of an informal settlement in Dar es Salaam, Tanzania : an empirically informed concept. In D.A. Swayne, W. Yang, A.A. Voinov, A. Rizzoli, and T. Filatova., editors, International Environmental Modelling and Software Society, iEMSs, page 8, Ottawa, 
Canada, 5-7 July 2010. 2010 International Congress on Environmental Modelling and Software Modelling for Environment's Sake.

[74] U.S.EPA. Projecting land use change: A summary of models for assessing the effects of community growth and change on land use pattern. In D.A. SWAYNE, W. Yang, A.A. Voinov, A. Rizzoli, and T. Filatova, editors, International Environmental Modelling and Software Society, iEMSs, page 260, Office of Research and Development, Cincinnati, OH, 5-7 July 2000. U.S. Environmental Protection Agency.

[75] Remy Sietchiping. A Geographic Information Systems and Cellular Automata-Based Model of Informal Settlement Growth. PhD thesis, School of Anthropology, Geography and Environmental Studies The University of Melbourne, Australia, April 2004.

[76] Amelie Schmolke, Pernille Thorbek, Donald L. DeAngelis, and Volker GRIMM. Ecological models supporting environmental decision making: a strategy for the future. Trends in Ecology and Evolution, 25(8):479 - 486, 2010.

[77] Claudia Werker and Thomas Brenner. Empirical Calibration of Simulation Models. Papers on Economics and Evolution 2004-10, Max Planck Institute of Economics, Evolutionary Economics Group, Jena, Germany, May 2004.

[78] Paul Windrum, Giorgio Fagiolo, and Alessio Moneta. Empirical Validation of Agent-Based Models: Alternatives and Prospects. Journal of Artificial Societies and Social Simulation, 10(2):8, 2007.

[79] Rosanna Garcia, Paul Rummel, and John Hauser. Validating agent-based marketing models through conjoint analysis. Journal of Business Research, 60(8):848-857, August 2007.

[80] J. Stoler, D. Daniels, J. R. Weeks, D. A. Stow, L. L. Coulter, and B. K. Finch. Assessing the Utility of Satellite Imagery with Differing Spatial Resolutions for Deriving Proxy Measures of Slum Presence in Accra, Ghana. GIsci Remote Sens, 49(1):31-52, 2012.

[81] Ellen-Wien Augustijn-Beckers, Johannes Flacke, and Bas Retsios. Simulating informal settlement growth in Dar es Salaam, Tanzania: An agent-based housing model. Computers, Environment and Urban Systems, 35(2):93-103, March 2011. 
[82] Odunuga Olaniyi Vincent. Exploring spatial growth pattern of informal settlements through Agent-based simulation. Master's thesis, International Institute for GeoInformation Science and Earth Observation, Enschede, Netherlands, March 2009.

[83] Volker Grimm, Eloy Revilla, Uta Berger, Florian Jeltsch, Wolf M. Mooij, Steven F. Railsback, Hans-Hermann Thulke, Jacob Weiner, Thorsten Wiegand, and Donald L. DeAngelis. Pattern-Oriented Modeling of Agent-Based Complex Systems: Lessons from Ecology. Science, 310(5750):987-991, 2005.

[84] J BARros. Urban growth in Latin American cities: exploring urban dynamics through agent-based simulation. PhD thesis, Bartlett School of Architecture and Planning, University of London, UK, October 2004.

[85] Joana Barros. Exploring Urban Dynamics in Latin American Cities Using an Agent-Based Simulation Approach. In Alison J. Heppenstall, Andrew T. Crooks, Linda M. See, and Michael Batty, editors, Agent-Based Models of Geographical Systems, pages 571-589. Springer Netherlands, 2012.

[86] M. Felsen and U. Wilensky. NetLogo Urban Suite - Economic Disparity model. Center for Connected Learning and Computer-Based Modeling, Northwestern Institute on Complex Systems, Northwestern University, Evanston, IL, 2007.

[87] M. Birkin, G. Clarke, M. P. Clarke, and A. Wilson. Intelligent GiS: Location Decisions and Strategic Planning. GeoInformation International, Cambridge, UK, 1996.

[88] Xia Li and Anthony Gar-On Yeh. Modelling sustainable urban development by the integration of constrained cellular automata and GIS. International Journal of Geographical Information Science, 14(2):131-152, 2000.

[89] M.S.A. RAO. Urban sociology in India. Texts and readings in the social sciences. Orient Longman, New Delhi, India, 1992.

[90] Robert E. Park. The City: Suggestions for the Investigation of Human Behavior in the City Environment. American Journal of Sociology, 20(5):577-612, 1915.

[91] Louis Wirth. Urbanism as a Way of Life. American Journal of Sociology, 44(1):124, 1938. 
[92] Sonalde Desai and Amaresh Dubey. Caste in 21st century India: competing narratives. Economic and political weekly, 46(11):40, 2012.

[93] Fred Arnold Kamla Gupta and H. Lhungdim. Health and Living Conditions in Eight Indian Cities. National Family Health Survey (NFHS-3), 2005-06, 2009.

[94] Surinder K. Mehta. Patterns of Residence in Poona (India) by Income, Education, and Occupation (1937-65). American Journal of Sociology, 73(4):496-508, 1968.

[95] N. Jayapalan. Rural Sociology. Atlantic Publishers \& Distributors (P) Limited, New Delhi, India, 2002.

[96] Veronique Dupont. Socio-spatial differentiation and residential segregation in Delhi: a question of scale? Geoforum, 35(2):157-175, 2004.

[97] Trina Vithayathil and Gayatri Singh. Spaces of Discrimination. Economic 8 Political Weekly, 47(37):60-66, 2012.

[98] ISA Baud, N Sridharan, and Karin Pfeffer. Mapping urban poverty for local governance in an Indian mega-city: the case of Delhi. Urban Studies, 45(7):1385$1412,2008$.

[99] Isa SA Baud, Karin Pfeffer, Namperumal Sridharan, and Navtej Nainan. Matching deprivation mapping to urban governance in three Indian megacities. Habitat International, 33(4):365-377, 2009.

[100] Vinodia Ajay Kumar, Sanjeev Singh, and Yogesh Garg. Tenability Scanner: A theoretical Framework for Classification of Slums in India. International Research Journal of Social Sciences, 4(7):70 - 77, 2015.

[101] Sumila Gulyani, Ellen M. Bassett, and Debabrata Talukdar. A tale of two cities: A multi-dimensional portrait of poverty and living conditions in the slums of Dakar and Nairobi. Habitat International, 43:98 - 107, 2014.

[102] Amit Patel, Naoru Koizumi, and Andrew Crooks. Measuring slum severity in Mumbai and Kolkata: A household-based approach. Habitat International, 41:300 - 306, 2014. 
[103] Davison Muchadenyika. Slum upgrading and inclusive municipal governance in Harare, Zimbabwe: New perspectives for the urban poor. Habitat International, 48:1 - 10, 2015.

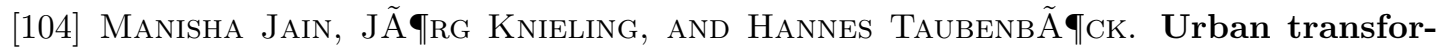
mation in the National Capital Territory of Delhi, India: The emergence and growth of slums? Habitat International, 48:87 - 96, 2015.

[105] Koen Olthuis, Jiya Benni, Kristin Eichwede, and Chris Zevenbergen. Slum Upgrading: Assessing the importance of location and a plea for a spatial approach. Habitat International, 50:270 - 288, 2015.

[106] Richard Tombinson. Scalable community-led slum upgrading: The Indian Alliance and community toilet blocks in Pune and Mumbai. Habitat International, 50:160 - 168, 2015.

[107] GWEN VAN EIJK. Unequal networks: spatial segregation, relationships and inequality in the city, 32. Gwen van Eijk, 2010.

[108] C. Tilly. Durable Inequality. University of California Press, California, US, 1999.

[109] Otis Dudley Duncan and Beverly Duncan. A methodological analysis of segregation indexes. American sociological review, pages 210-217, 1955.

[110] LingXin HaO AND ERIC Fong. Linking dichotomous segregation with multigroup segregation: Weighted segregation ratios in selected US metropolitan areas. Social science research, 40(1):379-391, 2011.

[111] Hervé Abdi and Michel Béra. Correspondence analysis. In Encyclopedia of Social Network Analysis and Mining, pages 275-284. Springer, 2014.

[112] Yan Kestens, Francois Des Rosiers, et al. Links between Residential Choice Criteria and Property Values. Some Evidence Using Correspondance Analysis and Hedonic Modelling. Technical report, European Real Estate Society (ERES), 2004.

[113] William AV Clark, MC Deurloo, and FM Dieleman. Modeling categorical data with chi square automatic interaction detection and correspondence analysis. Geographical Analysis, 23(4):332-345, 1991. 
[114] Bako Zachariah Zinas and Mahmud Bin Mohd Jusan. Housing choice and preference: Theory and measurement. Procedia-Social and Behavioral Sciences, 49:282-292, 2012.

[115] Mark O Hill. Correspondence analysis: a neglected multivariate method. Applied statistics, pages 340-354, 1974.

[116] Supriya RoyChowdhury. Livelihood and Income: Informality and Poverty in Bangalore's Slums, 2011.

[117] Anirudh Krishna, MS Sriram, and Purnima Prakash. Slum types and adaptation strategies: identifying policy-relevant differences in Bangalore. Environment and Urbanization, 26(2):568-585, 2014.

[118] N Krishnaji. Poverty and family size. Social Scientist, pages 22-35, 1980.

[119] RANJAn Ray. Poverty, household size and child welfare in India. Economic and Political Weekly, pages 3511-3520, 2000.

[120] Adriana D Kugler and Santosh Kumar. Preference for boys, family size and educational attainment in India. Technical report, National Bureau of Economic Research, 2015.

[121] Pramila Rai, Ishwari Sharma Paudel, Anup Ghimire, Paras Kumar Pokharel, Raju Rijal, and Surya Raj Niraula. Effect of gender preference on fertility: cross-sectional study among women of Tharu community from rural area of eastern region of Nepal. Reproductive Health, 11:15, 2014.

[122] Women and Karnataka Child Development. Bhagyalakshmi Scheme, 2006.

[123] Rockefeller Foundation. Constrained Opportunities in Economies, 2013.

[124] Anirudh Krishna. Stuck in Place: Investigating Social Mobility in 14 Bangalore Slums. The Journal of Development Studies, 49(7):1010-1028, 2013.

[125] Melanie Montgomery and Carey Curtis. Housing mobility and location choice: a review of the literature. Impacts of Transit Led Development in a New Rail Corridor, 2006. 
[126] W. O. Bearden and R. P. Wilder. Household life-cycle effects on consumer wealth and well-being for the recently retired. Journal of Macromarketing, 27(4):389-403, 2007.

[127] J. P. Carpenter, A. G. Daniere, and L. M. Takahashi. Cooperation. trust, and social capital in southeast asian urban slums, Journal of Economic Behavior E Organization, 55(4):533-551, 2004.

[128] K. McCarthy. Household life cycle and housing choices. Papers of the Regional Science Association, 37(1):55-80, 1976.

[129] D. KIRK. Demographic transition theory. Population Studies, 50(3):361-387, 1996.

[130] M. Murphy. Long-term effects of the demographic transition on family and kinship networks in britain. Population and Development Review, 37:55-80, 2011.

[131] N. G. Becker, K. Glass, Z. Li, and G. K. Aldis. Controlling emerging infectious diseases like \{SARS\}. Mathematical Biosciences, 193(2):205-221, 2005.

[132] F. Ball and P. Neal. A general model for stochastic \{SIR\} epidemics with two levels of mixing. Mathematical Biosciences, 180(1-2):73-102, 2002.

[133] R. R. Rindfuss, S. J. Walsh, B. L. Turner, J. Fox, and V. Mishra. Developing a science of land change: Challenges and methodological issues. Proceedings of the National Academy of Sciences of the United States of America, 101(39):13976-13981, 2004.

[134] P. Ranade. Population Dynamics in India. Ashish Publishing House, New Delhi, India, 1990.

[135] Jefferson Fox. People and the Environment, 1 of Approaches for Linking Household and Community Surveys to Remote Sensing and GIS. Springer Science \& Business Media, 2003.

[136] J. Liu, G. C. Daily, P. R. Ehrlich, and G. W. Luck. Effects of household dynamics on resource consumption and biodiversity. Nature, 421(6922):530-533, 2003.

[137] J. C. Allen and D. F. Barnes. The causes of deforestation in developing countries. Annals of the Association of American Geographers, 75(2):163-184, 1985. 
[138] D. L. Skole, W. H. Chomentowski, W. A. Salas, and A. D. Nobre. Physical and human dimensions of deforestation in amazonia. Bioscience, 44(5):314-322, 1994.

[139] BL Turner II, Jacqueline Geoghegan, and David R Foster. Integrated landchange science and tropical deforestation in the southern Yucatán: Final frontiers. Oxford University Press on Demand, 2004.

[140] J. Stillwell and M. Clarke. Population Dynamics and Projection Methods. Understanding Population Trends and Processes. Springer, London, UK, 2011.

[141] M. Spielauer. What is social science microsimulation? Social Science Computer Review, 29(1):9-20, 2011.

[142] A. R. Pebley. Demography and the environment. Demography, 35(4):377-389, 1998.

[143] Héctor A. López-Ospina, Francisco J. Martínez, and Cristián E. Cortés. Microeconomic model of residential location incorporating life cycle and social expectations. Computers, Environment and Urban Systems, 55:33-43, 2016.

[144] J. Van Rooyen and P. Du Plessis. A conceptual and empirical validation of the household lifecycle concept in south africa. SA Journal of Industrial Psychology, 29(3):30, 2003.

[145] F. Ball and P. Neal. Network epidemic models with two levels of mixing. Mathematical Biosciences, 212(1):69-87, 2008.

[146] F. Ball, D. Mollison, and G. Scalia-Tomba. Epidemics with two levels of mixing. The Annals of Applied Probability, 7(1):46-89, 1997.

[147] F. Ball and P. Neal. A general model for stochastic \{SIR\} epidemics with two levels of mixing. Mathematical Biosciences, 180(12):73-102, 2002.

[148] K. Glass, J. MCCAW, and J. MCVernon. Incorporating population dynamics into household models of infectious disease transmission. Epidemics, 3(34):152$158,2011$. 
[149] Neil M Ferguson, Derek AT Cummings, Simon Cauchemez, Christophe Fraser, Steven Riley, Aronrag Meeyai, Sopon Iamsirithaworn, and DonALD S BURKE. Strategies for containing an emerging influenza pandemic in Southeast Asia. Nature, 437(7056):209-214, 2005.

[150] D. Ettema, K. Jong, H. Timmermans, and A. Bakema. Puma: Multi-agent modelling of urban systems. In J. Stillwell Koomen, A. BAKema, AND H. Scholten, editors, Modelling Land-Use Change, pages 237-258, Springer, Netherlands, 2007. The GeoJournal Library.

[151] Ellen-Wien Augustijn-Beckers, Johannes Flacke, and Bas Retsios. Simulating informal settlement growth in dar es salaam, tanzania: An agent-based housing model. Computers, Environment and Urban Systems, 35(2):93-103, 2011.

[152] N. Geard, J. M. McCaw, A. Dorin, K. B. Korb, and J. McVernon. Synthetic population dynamics: A model of household demography. Journal of Artificial Societies and Social Simulation, 16(1):8, 2013.

[153] Cristina Montañola Sales. Large-scale simulation of population dynamics for socio-demographic analysis.

[154] J. P. Holdren and P. R. Ehrlich. Human Population and the Global Environment: Population growth, rising per capita material consumption, and disruptive technologies have made civilization a global ecological force. American scientist, 62(3):282-292, 1974.

[155] Volker Grimm, Uta Berger, Donald L. DeAngelis, J. Gary Polhill, Jarl Giske, and Steven F. Railsback. The \{ODD $\}$ protocol: A review and first update. Ecological Modelling, 221(23):2760 - 2768, 2010.

[156] A. Rathor. Slum Dwellers, Curse on Development. Sarup \& Sons, New Delhi, India, 2003.

[157] R. RAO. Social Organisation in an Indian Slum. Study of a Caste Slum. Mittal Publications, New Delhi, India, 1990.

[158] Andrea Saltelli. Variance based sensitivity analysis of model output. Design and estimator for the total sensitivity index. Computer Physics Communications, 181(2):259 - 270, 2010. 
[159] I.M SoвоL. Global sensitivity indices for nonlinear mathematical models and their Monte Carlo estimates. Mathematics and Computers in Simulation, 55(1):271 $-280,2001$.

[160] Andrea Saltelli. Making best use of model evaluations to compute sensitivity indices. Computer Physics Communications, 145(2):280 - 297, 2002.

[161] T. Egondi, C. Kabiru, D. Beguy, M. Kanyiva, and R. Jessor. Adolescent homeleaving and the transition to adulthood a psychosocial and behavioural study in the slums of nairobi. International journal of behavioral development, 37(4):298$308,2013$.

[162] A. Aassve, F. C. Billari, and F. Ongaro. The impact of income and employment status on leaving home: Evidence from the italian echp sample. Labour, 15(3):501-529, 2001.

[163] UNICEF. Policy advocacy and partnership for children's rights: Adolescents from the slums equipped to realise their dreams, March 2015.

[164] UN-HABITAT. The Challenge of Slums. Global Report on Human Settlements. Taylor \& Francis, 2012.

[165] Somik V. Lall, Mila Freire, Belinda Yuen, Robin Rajack, and Jean-Jacques HeLluin. Urban land markets: Improving land management for successful urbanization. Springer Science \& Business Media, Netherlands, 2009.

[166] T. Patel. The Family in India: Structure and Practice, Themes in Indian sociology. Sage Publications, New Delhi, India, 2005.

[167] Moshe E Ben-Akiva and Steven R Lerman. Discrete choice analysis: theory and application to travel demand, 9. MIT press, 1985.

[168] J de Ortuzar and Luis G Willumsen. Modelling Transport. Wiley, 2011.

[169] E.R. Dugundu and L. Gulyas. Sociodynamic discrete choice applied to travel demand: Multi-agent based simulation and issues in estimation. In Computer Science and Information Systems (FedCSIS), 2012 Federated Conference on, pages 12471254, Sept 2012. 
[170] Andrew B Cooper and Joshua J Millspaugh. The application of discrete choice models to wildlife resource selection studies. Ecology, 80(2):566-575, 1999.

[171] Mandy Ryan, Karen Gerard, and Mabel Amaya-Amaya. Using discrete choice experiments to value health and health care. Springer, 2008.

[172] Qijun Zhang and Wander Jager. Agent based modeling of population dynamics in municipalities: Migration in the Derbyshire \& Nottinghamshire cases in the UK. Technical report, University of Groningen, Center for Social Complexity Studies, December 2011.

[173] Cristian Guevara and Moshe Ben-Akiva. Endogeneity in residential location choice models. Transportation Research Record: Journal of the Transportation Research Board, 60(1977):60-66, 2006.

[174] Daniel McFadden. Modeling the choice of residential location. Transportation Research Record, 60(673), 1978.

[175] JoAn Leslie Walker. Extended discrete choice models: integrated framework, flexible error structures, and latent variables. PhD thesis, Massachusetts Institute of Technology, 2001.

[176] JoAn L WaLker and JiEPING Li. Latent lifestyle preferences and household location decisions. Journal of Geographical Systems, 9(1):77-101, 2007.

[177] Ricardo Hurtubia, My Hang Nguyen, Aurélie Glerum, and Michel Bierlaire. Integrating psychometric indicators in latent class choice models. Transportation Research Part A: Policy and Practice, 64:135-146, 2014.

[178] Guy H Orcutt. A new type of socio-economic system. The review of economics and statistics, pages 116-123, 1957.

[179] Moshe E Ben-Akiva and John L Bowman. Activity based travel demand model systems. In Equilibrium and advanced transportation modelling, pages 27-46. Springer, 1998.

[180] Birgit Muller, Friedrich Bohn, Gunnar Drebler, Jurgen Groeneveld, Christian Klassert, Romina Martin, Maja Schluter, Jule Schulze, Hanna Weise, and Nina Schwarz. Describing human decisions in agent-based models 
- ODD + D, an extension of the $\{$ ODD $\}$ protocol. Environmental Modelling and Software, 48:37 - 48, 2013.

[181] W. Alonso. Location and Land Use: Toward a General Theory of Land Rent. Publication of the Joint Center for Urban Studies. Harvard University Press, 1964.

[182] Alan W Evans. The economics of residential location. Springer, 1973.

[183] Harry W. Richardson. A generalization of residential location theory. Regional Science and Urban Economics, 7(3):251 - 266, 1977.

[184] John M Quigley. Consumer choice of dwelling, neighborhood and public services. Regional Science and Urban Economics, 15(1):41-63, 1985.

[185] Hoang Huu Phe and Patrick Wakely. Status, quality and the other trade-off: Towards a new theory of urban residential location. Urban Studies, 37(1):7-35, 2000 .

[186] William Clark, Marinus Deurloo, and Frans Dieleman. Residential mobility and neighbourhood outcomes. Housing Studies, 21(3):323-342, 2006.

[187] Alison Parkes, Ade Kearns, and Rowland Atkinson. What makes people dissatisfied with their neighbourhoods? Urban studies, 39(13):2413-2438, 2002.

[188] George C Galster and Anna M Santiago. What's the'hood got to do with it? Parental perceptions about how neighborhood mechanisms affect their children. Journal of Urban Affairs, 28(3):201-226, 2006.

[189] Sheilah Meikle, Tamsin Ramasut, and Julian Walker. Sustainable urban livelihoods: Concepts and implications for policy.

[190] John Farrington, Tamsin Ramasut, and Julian Walker. Sustainable Livelihoods Approaches in Urban Areas: General Lessons, with Illustrations, 2002.

[191] P.H. Rossi. Why families move. Sage Publications, 1980.

[192] William AV Clark and Jun L Onaka. Life cycle and housing adjustment as explanations of residential mobility. Urban studies, 20(1):47-57, 1983. 
[193] Tae-Kyung Kim, Mark W Horner, and Robert W Marans. Life cycle and environmental factors in selecting residential and job locations. Housing Studies, 20(3):457-473, 2005.

[194] Miller McPherson, Lynn Smith-Lovin, and James M Cook. Birds of a feather: Homophily in social networks. Annual review of sociology, pages 415-444, 2001.

[195] Kelly A Mollica, Barbara Gray, and Linda K Trevino. Racial homophily and its persistence in newcomers' social networks. Organization Science, 14(2):123136, 2003.

[196] Jacqueline J. Brown and Peter H. Reingen. Social Ties and Word-of-Mouth Referral Behavior. The Journal of Consumer Research, 14(3):350-362, 1987.

[197] Diane Archer. Social capital and participatory slum upgrading in Bangkok, Thailand. PhD thesis, Department of land economy, University of Cambridge, October 2010.

[198] Petr Matous and Kazumasa Ozawa. Measuring Social Capital in a Philippine Slum. Field Methods, 22(2):133-153, 2010.

[199] S. Wasserman and K. Faust. Social Network Analysis: Methods and Applications. Structural Analysis in the Social Sciences. Cambridge University Press, 1994.

[200] S. Bandyopadhyay, A.R. Rao, and B.K. Sinha. Models for Social Networks With Statistical Applications. Advanced Quantitative Technology in the Social Sciences. SAGE Publications, 2011.

[201] Chris Shughrue. A Model of Nonlinear Urbanization and Information Flows Across India. Hixon Center for Urban Ecology, 1(1):1-19, 2013.

[202] Matthew O. Jackson, Tomas Rodriguez-Barraquer, and Xu Tan. Social Capital and Social Quilts: Network Patterns of Favor Exchange. American Economic Review, 102(5):1857-1897, 2012.

[203] Robin I. M. Dunbar. The social brain hypothesis. Evolutionary Anthropology: Issues, News, and Reviews, 6(5):178-190, 1998. 